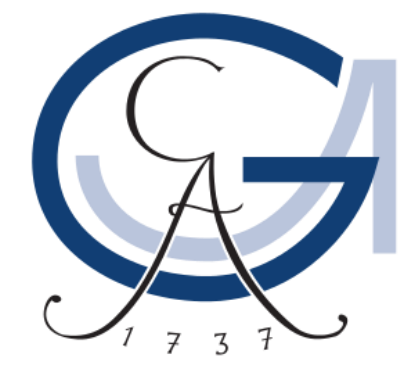

\title{
Binary Planet-Satellite Nanostructure Using RAFT Polymer
}

\author{
Dissertation
}

Wentao Peng

from Jiaxing, China

February 2020

Göttingen 



\section{GEORG-AUGUST-UNIVERSITÄT GÖTTINGEN}

\section{Binary Planet-Satellite Nanostructure Using RAFT Polymer}

\section{Dissertation}

zur Erlangung des mathematisch-naturwissenschaftlichen Doktorgrades

"Doctor rerum naturalium"

der Georg-August-Universität Göttingen

im Promotionsprogramm Gauss

der Georg-August University School of Science (GAUSS)

vorgelegt von

Wentao Peng

aus Jiaxing, China

Göttingen, 2020 



\section{Betreuungsausschuss}

Prof. Dr. Philipp Vana, MBA Institut für Physikalische Chemie Georg-August Universität Göttingen

Prof. Dr. Alec Wodtke

Institut für Physikalische Chemie Georg-August Universität Göttingen

\section{Mitglieder der Prüfungskommission}

\section{Referent}

Prof. Dr. Philipp Vana, MBA Institut für Physikalische Chemie Georg-August Universität Göttingen

\section{Korreferent}

Prof. Dr. Alec Wodtke

Institut für Physikalische Chemie Georg-August Universität Göttingen

\section{Weitere Mitglieder der Prüfungskomission}

Prof. Dr. Michael Buback

Prof. Dr. Ricardo Mata

Prof. Dr. Andreas Janshoff

Prof. Dr. Sven Schneider

Tag der mündlichen Prüfung
Institut für Physikalische Chemie Georg-August Universität Göttingen

Institut für Physikalische Chemie Georg-August Universität Göttingen

Institut für Physikalische Chemie Georg-August Universität Göttingen

Institut für Physikalische Chemie Georg-August Universität Göttingen

18.03.2020 

"My heart is like a satellite of yours, round round, and around you. J"

T. Ishino 



\begin{abstract}
This thesis provides a series of strategies to fabricate hierarchically arranged nanohybrids from distinct nanoparticles using polymer linkers. Benefit from the controlled reversible-deactivation radical polymerization technique, the topology and size of the polymer linker can be precisely controlled, which further enables full control over the size and architecting of the binary nanostructures. Reversible addition-fragmentation chain transfer (RAFT) polymerization was employed to synthesize polymers with narrow molecular weight distribution while inherently carrying the strong aurophilic and argentophilic trithiocarbonate moieties on their chain termini.
\end{abstract}

Start on the basis of the strong affinity of RAFT terminated polymer to the surface of gold and silver, the gold-planet-silver-satellite nanostructures are fabricated by firstly creating four-arms star RAFT polymer capped gold nanoparticles as a globular scaffold using a grafting-to approach. The multi-arm design of the polymer enables further linkage to the silver nanoparticles yielding a planet-satellite-like nanostructure. The strengths of this approach include the fine-tuning of interparticle distances on the nanometer scale by tailoring the size of the star polymer linker. The gold-planet-silver-satellite nanostructure possesses significant plasmonic coupling phenomena making it potentially a very powerful tool for surfaceenhanced Raman application.

This thesis focuses further on the creation of binary planet-satellite nanoparticles with more distinct nanoparticles. This binary nanostructure is designed for the application of nanopatterning on a substrate. The final goal is to implement the highly ordered arrangement of both nanoparticles as a binary template for the cell-response experiment. Under the 
consideration of the chemical feasibility and the following biological accessibility, silica is chosen as the nano-planet while gold as the satellites. This thesis established a general and straightforward method to fabricate silica-planet-gold-satellite nanostructure with full control of the structural details, i.e., the number of satellites, interparticle distance, and the size of the satellite nanoparticles. In this approach, surface-initiated RAFT polymerization is implemented to obtain a well-defined polymer brush on the silica surface featuring trithiocarbonate moieties at the polymer chain termini which allows further attachment of noble metal nanoparticles. AuNP is chosen as the satellite nanocomponent due to its well-established protocols for the cell-response experiments in the previous studies. Transmission electron microscopy (TEM) shows that the silica-planet-goldsatellite nanostructure can self-assemble into a highly ordered hexagonal pattern on carbon film by simply drop-casting. The interparticle-distance between planet and satellite nanoparticles can be precisely controlled between 10 to $50 \mathrm{~nm}$ range which meets the design of the cell-response experiment. Based on these results, the polymer-based planet-satellite nano-assemblies have a very high potential to be applied as a unique and powerful template for creating a 2D binary bifunctional nanopattern. To unfold this feature, a method for producing a homogenous monolayer of the planet-satellite nanostructure on the $\mathrm{CaF}_{2}$ surface is established by using dip-coating technique. The polymer component is successfully removed by plasma treatment exposing the nanoparticles for further chemical or biological modification.

According to the cell experiments, $\mathrm{CaF}_{2}$ is found to be an unsuitable substrate for the desired task due to the insufficient passivation (PEGylation) toward the labeling peptide. A method of transferring both gold and $\mathrm{SiO}_{2}$ nanoparticles onto hydrogel is established by introducing specific linker molecules on gold and $\mathrm{SiO}_{2}$ nanoparticles and successive photopolymerization with PEG-diacrylate. 
Furthermore, the RAFT-polymer functionalized nanoassemblies can quickly expend their utility by taking advantage of its inherent flexibility in the design of the polymer structure and nano component. Two distinct strategies are applied to build new features into the nanoassemblies. The first strategy uses RAFT/thiol terminated polyethylene to create an efficient synthesis route for the self-assembly of gold/silver-core-PE-shell nanohybrids. This nanohybrids successfully inherit the unique solution behavior of PE. The second approach utilizes the microemulsion technique to achieve a one-to-one silica coating of $\sim 7 \mathrm{~nm}$ magnetite nanoparticles and introduces the favored superparamagnetism to the nanosystem. Owing to the silica coating and polymer shells, the magnetic interaction between each magnetite nanoparticle is effectively inhibited, which is highly favored in the realm of magnetic hyperthermia applications. 


\section{Table of Contents}

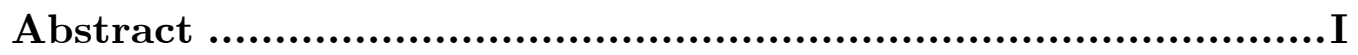

Part A Theoretical Background

Chapter 1 Tailoring Multifunctional Core-Satellite

Nanostructures: State of the Art......................................... 1

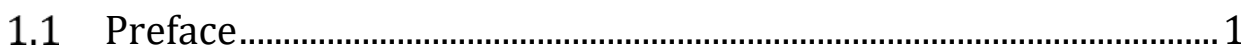

1.2 Approach with the molecular linker..................................................

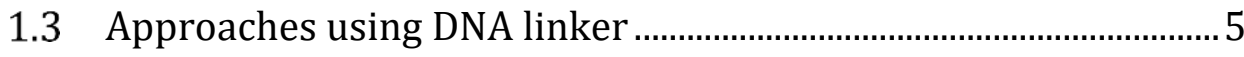

1.4 Approaches using polymer linker ……………………………..... 11

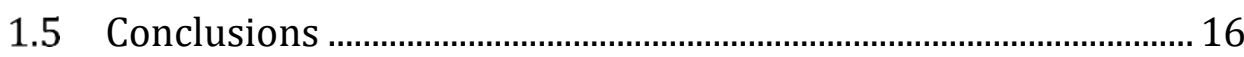

Chapter 2 Designing Binary Nanostructure: Methods and

Building Blocks ..............................................................19

2.1 Preface

2.2 Noble metal nanoparticles ........................................................... 20

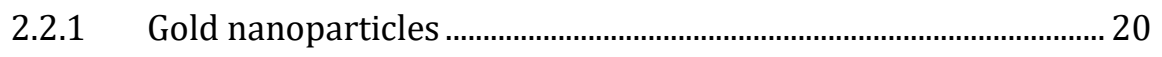

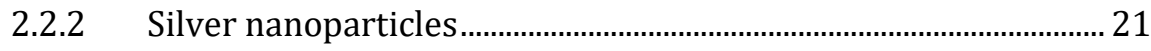

2.3 Synthesis and functionalization of silica nanoparticles............... 23

2.4 The approaches of anchoring polymer onto nanoparticles'

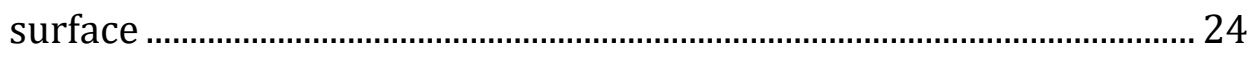

2.4.1 Star polymer capped AuNPs: grafting-to approach ........................ 25 
2.4.2 Surface-induced RAFT polymerization from silica: grafting-from

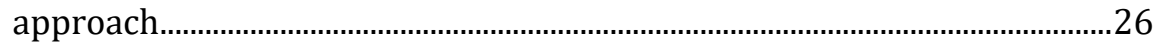

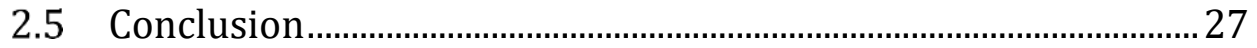

Chapter 3 Nanopatterning Mediated Cell-Responses ............ 29

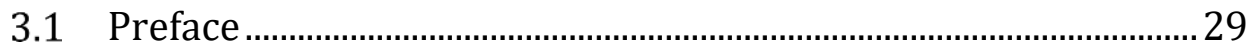

3.2 Nanopatterning: a brief state-of-the-art review ............................. 30 3.2.1 Block copolymer micelle nanolithography for cell response experiments 32

3.2.2 Challenges to fabricate binary planet-satellite nanopatterning for biological experiment 39

3.3 Conclusions 41

Part B Result and Discussion

Chapter 4 Gold-Planet-Silver-Satellite Nanostructures using RAFT Star Polymer ............................................................45

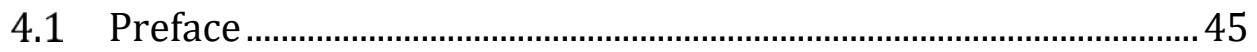

4.2 Self-assembly of gold-planet-silver-satellite nanostructures ... 47

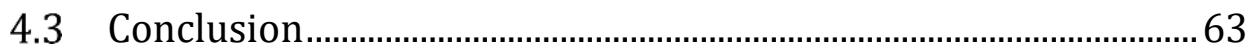

Chapter 5 Fabricating Silica-Planet-Gold-Satellite

Nanostructure for Binary Nano-Patterning ...........................65

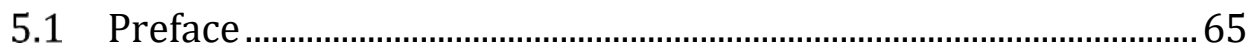

5.2 Nano-planet: surface-induced RAFT polymerization from silica

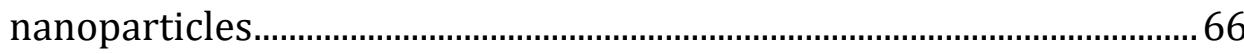


5.2.1 Introducing RAFT group onto the surface of $\mathrm{SiO}_{2}$ surface............ 67

5.2.2 Controlled surface-initiated polymerization from silica

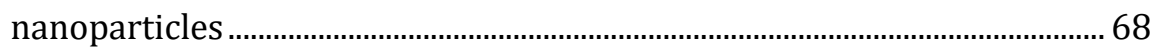

5.3 High-precision self-assembly of silica-planet-gold-satellite

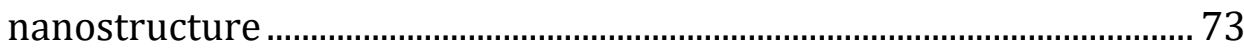

5.4 2D nano-patterning with the planet-satellite nanostructure ... 79

5.4.1 Monolayer formation of planet-satellite nanostructure on the

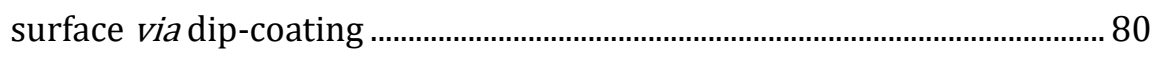

5.4.2 Removal of polymer content by plasma treatment........................ 88

5.4.3 Transfer of binary planet-satellite nanopattern onto the

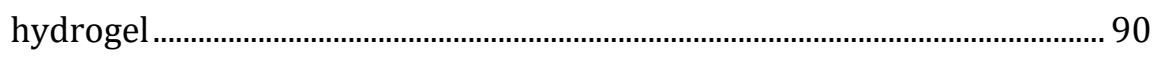

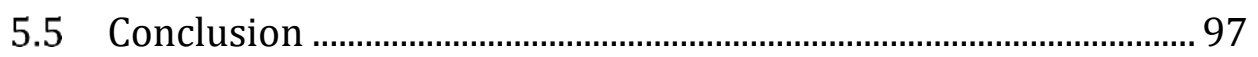

Chapter 6 Further Development of RAFT-Polymer

Functionalized Nanoparticle Assemblies ...........................99

6.1 Polyethylene-grafted gold and silver nanoparticles using

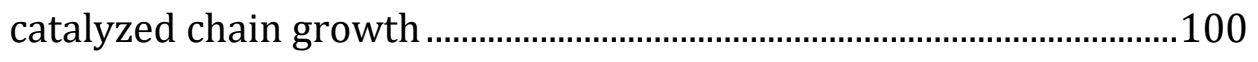

6.2 Silica-coated magnetite nanoparticles carrying a high-density

polymer brush shell of hydrophilic polymer..........................................106

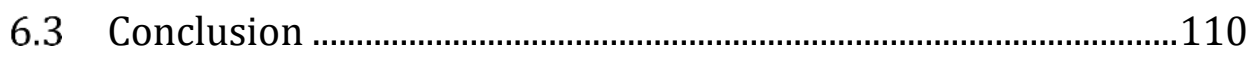

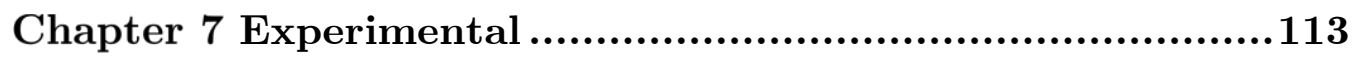

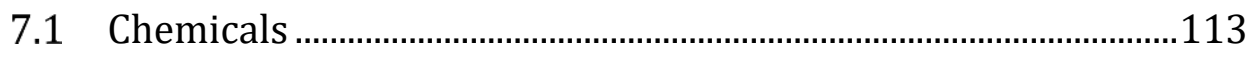

7.2 Equipment and analytical methods ............................................114 
7.3 Synthesis of nanoparticles ............................................................... 117

7.4 Synthesis of chemicals and polymerization's condition ........... 123

7.5 Grafting polymer onto AuNPs and AgNPs ................................... 129

7.6 Self-assembly of planet-satellite nano-structures ...................... 129

7.7 Preparation and experiments of planet-satellite nanostructure

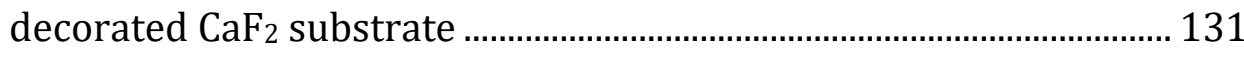

Chapter 8 Closing Remarks ............................................. 133

8.1 Conclusions .......................................................................................... 133

8.2 Future perspectives........................................................................ 135

Abbreviations and Symbols ............................................. 138

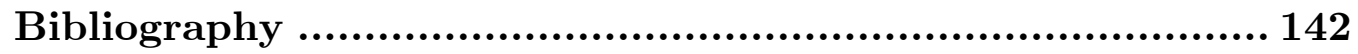

Acknowledgements....................................................... 157

Curriculum Vitae ........................................................ 159 


\section{Part A}

\section{Theoretical Background}




\section{Chapter 1}

\section{Tailoring Multifunctional Core-Satellite Nanostructures: State of the Art}

\subsection{Preface}

The development on the synthesis and the applications of the planetsatellite (or core-satellite) nanostructures have attracted enormous research interest in material science. Simultaneously integrating two distinct nano-units into one well-defined single nanostructure gives diverse hybrid characteristics for novel and unique applications in different fields. To accomplish a precise assembly of the planet-satellite nanostructure, various synthetic approaches based on the molecular linker, polymer linker, and DNA conjugation have made significant progress recently.

Among the various nanocomposites, gold and silver nanoparticles have a substantial impact in diverse fields including electronic,[1] optical,[2,3] sensing, ${ }^{[4]}$ catalysis,[5] energy conversion,[6] photochemistry,[7] nanopatterning,[8] bio-imaging,,9] drug delivery,[10] and medicines.[11] For most applications, surface modification is crucial for enhanced colloidal stability and facile functions. To this end, the modification of NPs with functional polymer is an efficient and powerful approach to introduce further favored properties (i.e., stimuli-responsiveness ${ }^{[12]}$ ) while enforcing the general stability of the nanostructure.

In general, carrying anchor groups with strong interaction towards the nanoparticles' surface is the major requirement for creating an effective linker to self-assembly the nanoparticles. For noble metal nanoparticles, sulfur-containing groups, i.e., thiol,[13] disulfides, ${ }^{[14]}$ RAFT group ${ }^{[15,16]}$ have strong interaction with the gold surface. The small molecular linker carrying 
multiple anchoring groups is the simplest tool to connect nanoparticles. The molecular linker often provides easy protocol while possessing limited function and spacing between naoncomponents. These anchoring groups can also be introduced to macromolecules as end-groups by localizing sulfur-containing moieties at the termini of the linker chain (e.g., inherently trithiocarbonate terminated RAFT polymer, ${ }^{[17]}$ (bi)thiol-modified DNA ${ }^{[18]}$ or polymer $\left.{ }^{[19]}\right)$. This termini design made polymer and DNA a flexible and accurate linker for the targeted fabrication of high precise nanostructures due to their tunable size (nm regime) and predeterminable detailed structuring design. It is worth mentioning that compared to the DNA conjugation-based approach, the polymeric linker is much more costeffective and easier to produce.

\subsection{Approach with the molecular linker}

Using short molecular linkers as building blocks can be easily considered as the most efficient and straightforward approach for fabricating coresatellite nanostructure with a simple arrangement. The general disadvantage of the molecular linker is the lack of distance between core and satellite components, which limits its potential for many applications compared with the macromolecular linker. However, the sub-nanometer interparticle distance could be favored in several applications, especially in the surface-enhanced Raman scattering probes, which requires strong surface plasmatic coupling between noble metal nanoparticles.[20]

The strategies for creating of core-satellite nanostructure using small molecules is versatile: electrostatic interactions,[20-22] metal-ion-mediated complexation ${ }^{[23,24]}$ and "click chemistry" ${ }^{[25]}$ could induce attachment of satellite nanoparticles onto the surface of core particles. In a typical protocol, a bi-functionalized molecular linker is used for surface modification of the nano-core, while exposing the other chain-end as the anchor termini for the linkage of satellite nanoparticles. The linkage between core and satellite 
nanoparticles should be chemically strong to hold the nanoparticles together. Figure 1.1[20] demonstrated the self-assembly of a $65 \mathrm{~nm}$ AuNP core with $35 \mathrm{~nm}$ AuNPs satellites based on electrostatic interaction between core and satellite nanoparticles. In this approach, the surface charge state on AuNPs was monitored by the termini of thiol-anchored molecules on core and satellite nanoparticles, respectively. The positive charges on the core AuNPs were created by amine moiety from $p$-aminothiophenol. To avoid the anchoring of amine side onto AuNPs, ${ }^{[26]}$ here, acidic $\mathrm{pH}$ value was required to prevent self-conjugations of the cores. The $\mathrm{pH}$ value of satellite nanoparticles was independently adjusted to 2.0 to lower the zeta-potential of the AuNPs, which made the satellites prone to aggregation. In this state, the satellite nanoparticles can interact with the cores upon mixing and selfassemble to the core-satellite nanostructure. The non-covalent linkage method requires a sensitive environment on the ionic strength, $\mathrm{pH}$ value and impurities of the system and the solvent for nanostructure is limited in pure water. The monitoring of zeta-potential is crucial to establish the successful assembly of the nanostructure.

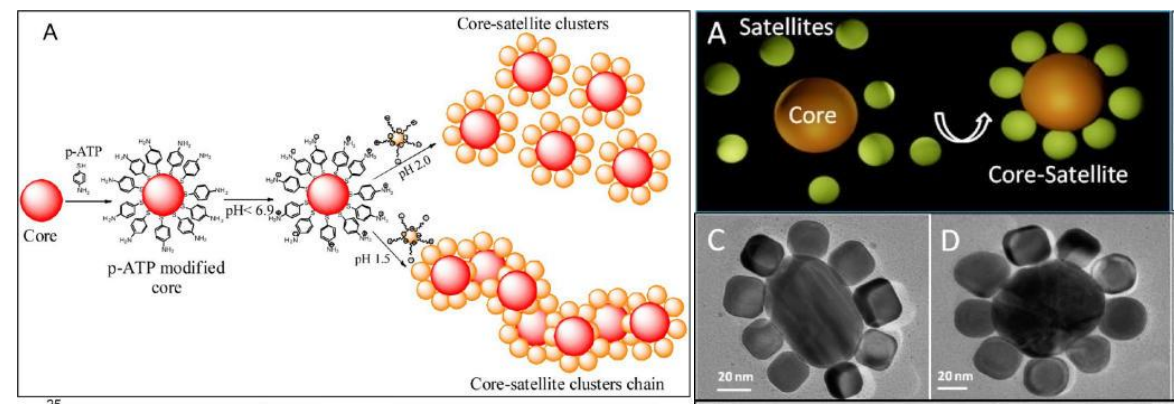

Figure 1.1: The left schema shows the fabrication strategy of gold-core-gold-satellite nanostructure based on electrostatic interaction. The right TE micrograph shows the actual structure of core-satellite nanostructure. Adapted with permission from ref. [20]. Copyright 2012 American Chemical Society.

Assembling core and satellite nanoparticles, especially for noble nanoparticles, using thiol is a very straightforward and flexible method. The strong RS-Au bonds[13] provides rapid and robust binding with a binding 
strength of approximately $170 \mathrm{~kJ} \cdot \mathrm{mol}^{-1[27]}$ and total net stabilization energy of roughly $-20 \mathrm{~kJ} \cdot \mathrm{mol}^{-1}$ considering the break of $\mathrm{S}-\mathrm{H}$ bond and formation of $\mathrm{H}-\mathrm{H}$ bond.[28] Figure 1.2 demonstrated a method to synthesis asymmetric gold-core-gold-satellite nanostructures using 1,10decanedithiol as a linker. The core AuNPs were first adsorbed onto an amine-functionalized glass surface using the amine-Au interaction [26]. The surface of core AuNPs was subsequently modified with 1,10-decanedithiol before the addition of satellite AuNPs. After the self-assembly, the planetsatellite nanostructure can be collected from the substrate with the aid of sonication while the excess of satellite AuNPs remaining attached to the substrate under legitimate sonication condition. In this approach, the distance between the planet and satellite was limited up to $3 \mathrm{~nm}$.
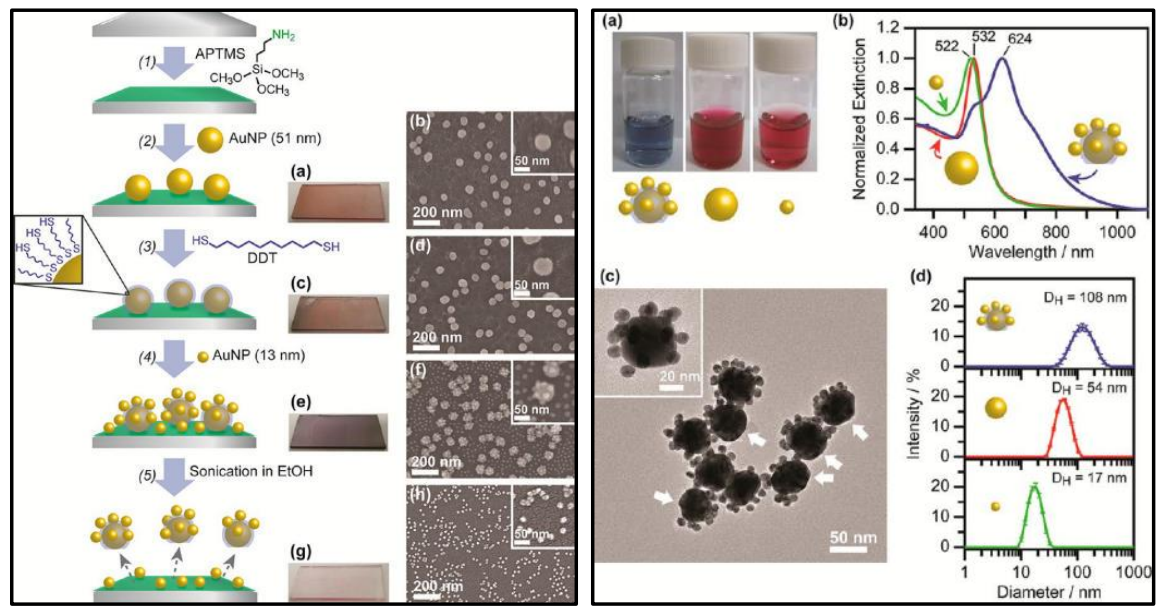

Figure 1.2: (Left) the assembly of asymmetric core-satellite nanostructure using bifunctionalized thiol as the linker. (Right) The core-satellite nanostructure possesses strong surface plasma coupling, which can be visualized by a strong redshift of UV-Vis absorption peak. Adapted with permission from ref. [29]. Copyright 2012 American Chemical Society.

To overcome the spatial limitation of molecular linker, i.e., to increase the distance between core and satellite NPs, a nano-thin silica layer can be introduced as a spacer to control the inter-particle distance and monitor the properties of the nanostructure (Figure 1.3).[30,31]These approaches turn the 
task of connecting metal-metal nanoparticles into the self-assembly of noble metal nanoparticles onto silica, which can be accomplished by modifying the surface with anchoring moiety (thiol or amine) via silane chemistry. ${ }^{[32]}$

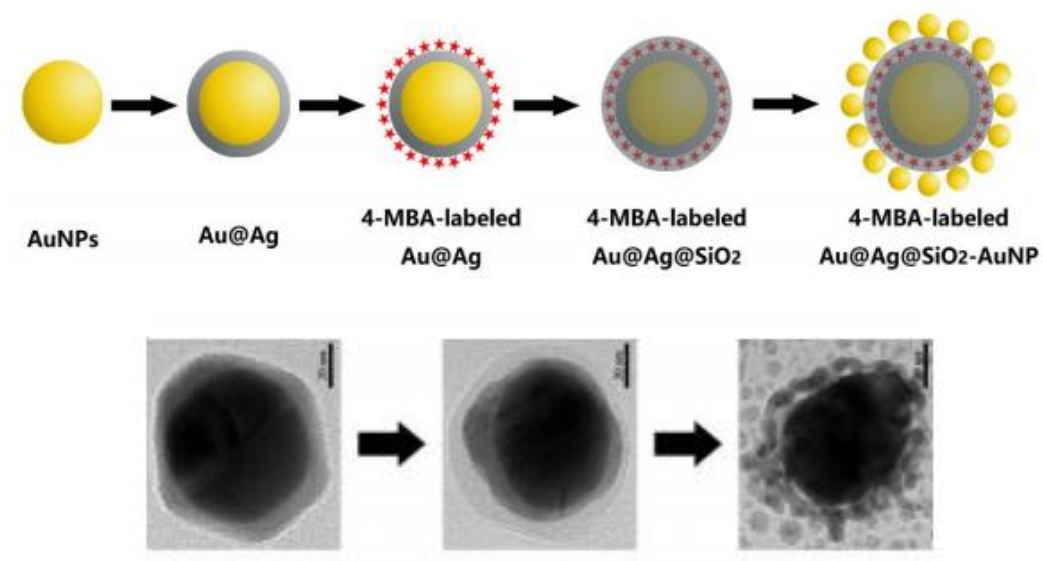

Figure 1.3: Assembly of Au@Ag@ $\mathrm{SiO}_{2}$ core with gold satellite. In the last step, (3aminopropyl) trimethoxysilane was introduced on the surface of silica for the further attachment of satellite AuNPs. 4-mercaptobenzoic acid (MBA) served as a Raman tag here. Adapted with permission from ref. [30]. Copyright 2019 American Chemical Society.

\subsection{Approaches using DNA linker}

To achieve a precise planet-satellite structure, synthetic strategies based on DNA conjugation leading to the self-assembly of nanomaterials has made significant progress recently and find many intriguing applications, especially for biological and clinical applications.

In this type of approach, the self-assembly of nano superstructure is initiated by mixing DNA-strands functionalized core nanoparticles with satellite nanoparticles, which is decorated with a complementary sequence. The DNA conjugation provides an effective driving force for the colloidal self-assembly process. 
In the early work, the gold planet-satellite assemblies were fabricated by using the thiol-terminated DNA oligonucleotide sequence (Figure 1.4) ${ }^{[33]}$. Briefly, the planet and satellite nanoparticles were functionalized with the corresponding DNA sequence ex situ in separated colloids and mixed forming the self-assembled nanostructure. In order to enhance the colloidal stability and lower the non-specific interactions with biomolecules and cells, ${ }^{[34]}$ thiol-terminated PEG was subsequently added to the mixture covering the outer side of the superstructure. Precise control over the number of satellites can be achieved by dosing the functionalized satellite nanoparticles. Furthermore, by building multiple specific binding sequences on the DNA linker, multi-layered nanostructures can also be obtained. It is worth mentioning that for the multi-layered nanostructure, the chain length of PEG-SH had a significant impact on the interparticle distance between satellite nanoparticles after drop-casting on the carbon film. The planetsatellite nanostructure showed advantages in mediating the biological delivery and elimination of nanoparticles, i.e., improving the accumulation into tumors and facilitate the elimination from the body. 

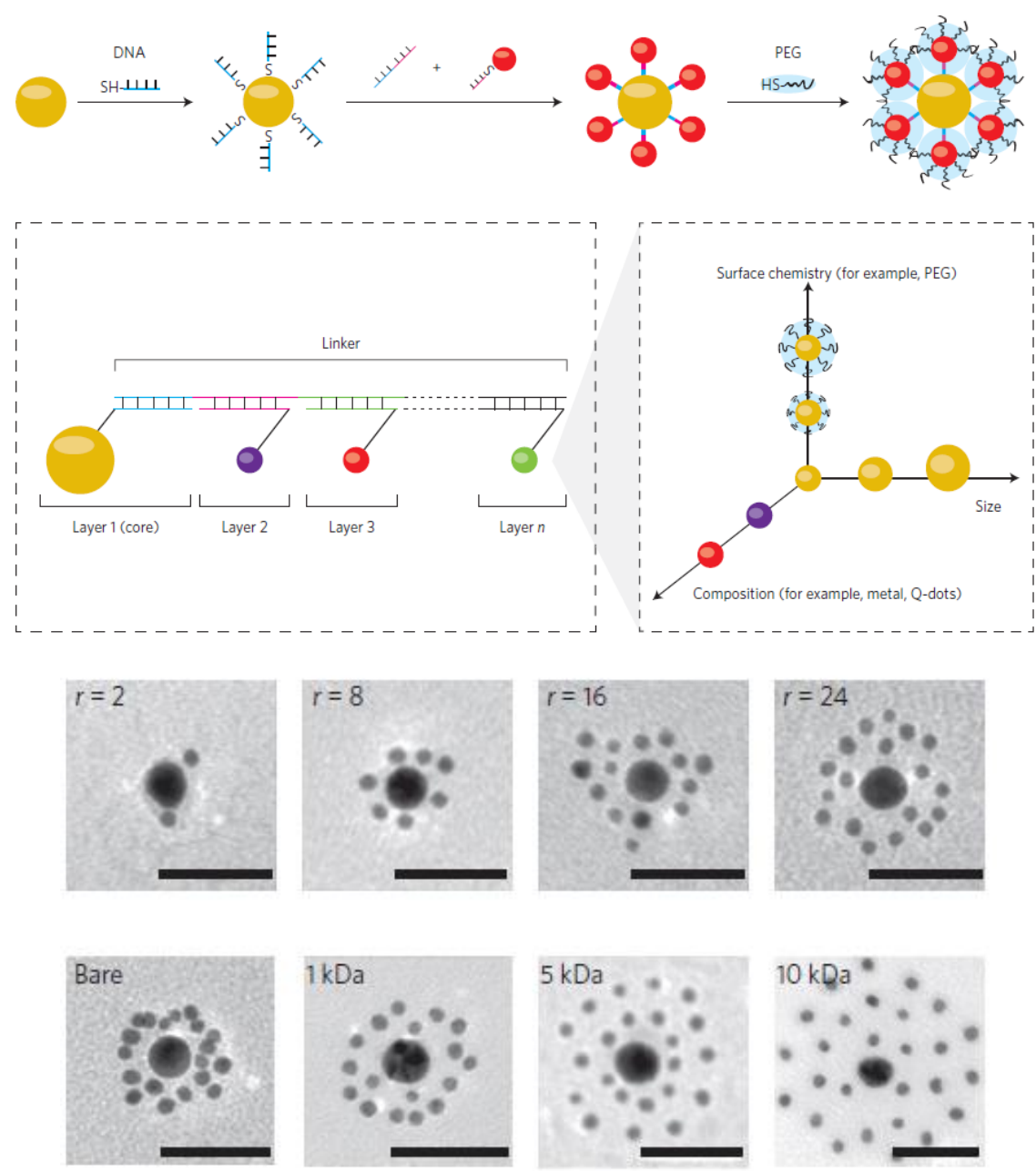

Figure 1.4: Fabrication strategy for the gold planet-satellite nanostructure using DNAconjugation. The number of satellite nanoparticle can be monitored by the dosing of satellite AuNPs. The multi-layered structure is obtained by building multiple binding sequences on DNA. Scale bars, $50 \mathrm{~nm}$. Adapted with permission from ref. [33]. Copyright 2014 Springer Nature.

DNA origami provided very intriguing strategies to assembling hierarchical nanostructures from various nanoparticles (Figure 1.5), ${ }^{[35]}$ e.g., metal nanoparticles, quantum dots, organic dyes. The high stiffness of the DNA origami structure enabled defined interparticle distance up to $200 \mathrm{~nm}$. However, this approach is that the synthesis is limited by the very high 
synthetic cost and poor yield of DNA origami units which restrained the application of this type of nanostructures.
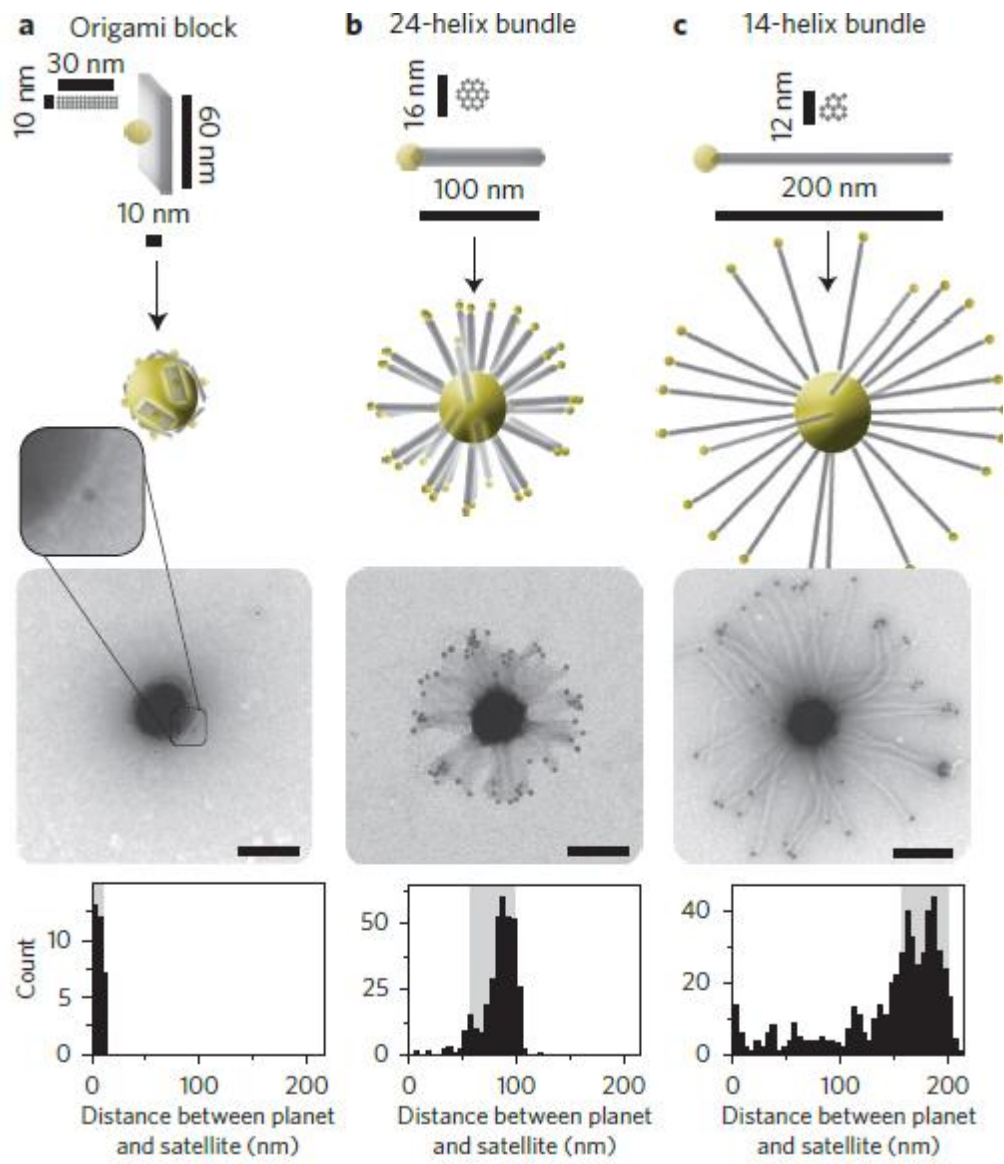

Figure 1.5: Planet-satellite distance control by varying the size of the DNA origami linker. Scale bar: $80 \mathrm{~nm}$. Adapted with permission from ref. [35]. Copyright 2014 Springer Nature.

Synthetic approaches based on DNA conjugation leading to the selfassembly of nanomaterials have made significant progress recently and find many exciting applications, especially for biological and clinical applications: $\mathrm{Xu}$ et al. has fabricated a core-satellite nano-assembly of gold and quantum dots for ultrasensitive micro RNA detection in vitro and in vivo.[36] In that work, the fluorescence of quantum dots ( $\mathrm{AgS}_{2}$, as satellites) is quenched by plasmonic gold nanoparticle (planet) at a closed nanometer range after 
linking with specific DNA sequences. The linker DNA sequence can capture targeted micro RNA and release free quantum dots emitting detectable fluorescence. Xu's group has also reported a photoactive planet-satellite structure with a platinum-coated Au nanorod as a planet and fluorescent $\mathrm{Ag}_{2} \mathrm{~S}$ as satellites, ${ }^{[37]}$ providing a sensitive detection of micro RNA and tumor diagnosis and therapy using similar targeting strategy. In that work, the Pt coating on Au nanorods induced a red-shift of plasmonic resonance to the NIR region offered advantages in terms of NIR photothermal therapy while $\mathrm{Ag}_{2} \mathrm{~S}$ as a powerful NIR fluorescence emitter.

Using a similar approach, Xu's group further fabricated nano-assemblies with gold nanorods dimer as a planet and upconverting nanoparticles (UCNPs) as satellites for enhanced phototherapy of tumor in vivo: the gold nanorod dimer serves as a powerful photothermal core, and the UCNPs are used for photodynamic treatment (Figure 1.6).[38] Due to the well-defined core-satellite nanostructure, the favorable functions of both nanocomponents were integrated into a single nano-platform, which is capable of enhancing the tumor therapeutic efficiency under safer power dosing by using both phototherapy approaches simultaneously. It is worth mentioning that, these authors also demonstrated that the encapsulation of amphiphilic polymer around the nanohybrid can enhance the biocompatibility and prevents the luminance quenching of the UCNPs for the plasmonic metal. In these works, ${ }^{[37,38]}$ the core-satellite nanostructure often provides access to multi-modal imaging (e.g., NIR fluorescence, CT, photoacoustic imaging) alongside their medical functions owing to the presence of AuNPs or fluorescence emitter. 

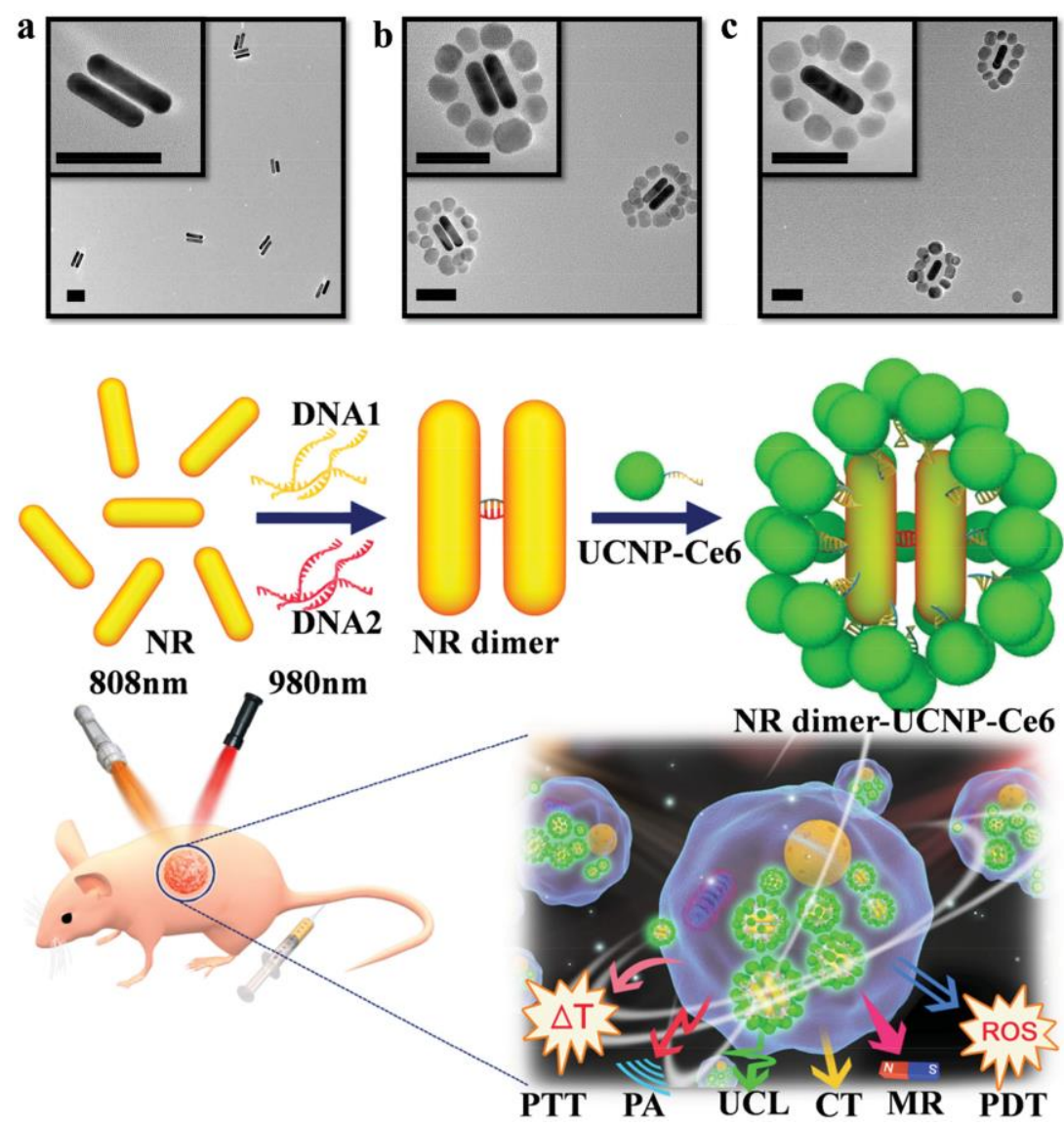

Figure 1.6: Upper: TEMicrograph of self-assembled Gold nanorods-NaGdF 4 nanostructure. a) Dimer of Au nanorods assembled by DNA. b) Dimer-Au-nanorodscore-NaGdF 4 -satellite nano-assemblies. c) Au-nanorods-core-NaGdF $\mathrm{N}_{4}$-satellite nanoassemblies, scale bars $50 \mathrm{~nm}$. Below: Schematic illustration of DNA-based Gold nanorods and upconversion nanoparticle assemblies for multimodal imaging-guided combination phototherapy. Adapted with permission from ref. [38]. Copyright 2016 Wiley-VCH Verlag GmbH \& Co. KGaA.

Cha et al. have also fabricated a DNA-assembled core-satellite upconverting-metal organic framework nanohybrid for photodynamic therapy in NIR wavelength. In that work, MOF generates singlet oxygen upon radiation of visible light, which is generated by upconverting nanoparticle upon NIR radiation. [39] The author demonstrated that under 
NIR irradiation, the MOF-core-UCNP-satellite structure was able to produce significantly more singlet oxygen than simply mixing UCNPs and MOF NPs.

In summary, DNA based core-satellite nanostructure has achieved numerous intriguing medical and biological applications in a prototype form. However, the commercial (clinic) commitment is limited by the mass production of the DNA component. Functional polymer with a well-designed structure can be a very cheap and efficient alternative for DNA in many applications. It is worth mentioning the inevitability of DNA linker for application as RNA detector due to its unique structure information. How to combine the character of both functional polymer (e.g., stimuli-responsive) and biological moieties (e.g., biological detection function from DNA, antibody) in a sophisticated and efficient manner should be the next necessary step for core-satellite nanostructure towards the clinical uses.

\subsection{Approaches using polymer linker}

Functional polymer with well-defined size and sophisticatedly designed structure opens up the feasibility of constructing a planet-satellite nanostructure. Choosing polymer as a central linking unit for this task has multiple advantages such as high colloidal stability owing to the steric shielding of a polymer shell, the tunable interparticle distance between planet-satellite, incorporating favorable characters from functional polymer and last but not least, its low cost. To this end, the flexibility in the macromolecular structure including topology, end-group functionality and the choice of monomer, offers very open design for creating hierarchical nanostructure with desired functions.

The most crucial challenge to design a polymeric nanoparticle linker is the topological structure of the polymers: the conventional linear design with one functional end-moiety does not have the competence for linking 
nanoparticles. Follow the line of this thought, self-assembly strategies based on multi-arms polymer was developed for this task.

The number of free end-group on the polymer functionalized core nanoparticle surface is crucial for the self-assembly process. One practical approach to increase the free end-group is the by using hyperbranched polymer carrying multiple anchoring end-groups (trithiocarbonate and alkyne, Figure 1.7, upper).[40] Though the hyperbranched polymer possesses a less precise structure, the nanostructure still presents a well-defined structural arrangement with a tunable number of satellite nanoparticles. The molecular weight of the hyperbranched polymer was rather low (approximately under $10 \mathrm{~kg} / \mathrm{mol}$ ), the interparticle distance was not observed in this case. However, the strong surface-enhanced Raman (SER) effect can be easily observed and monitored by several attached gold nanoparticles. (Figure 1.7, lower right)
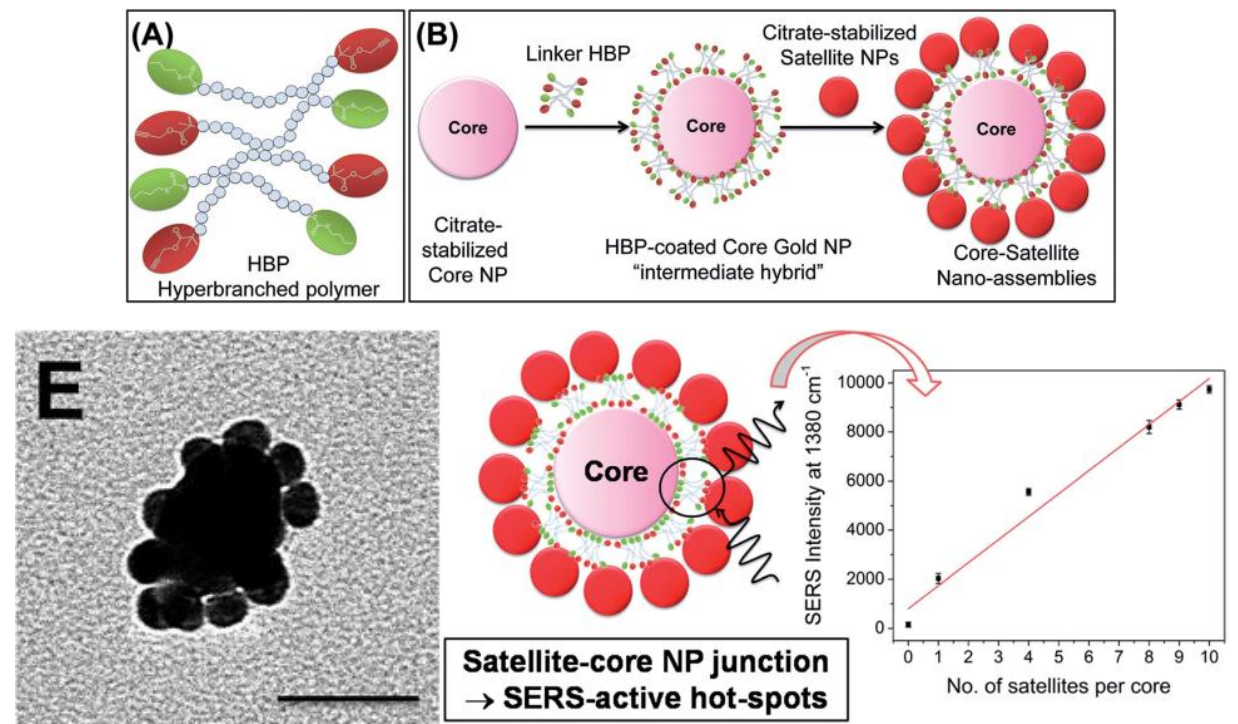

Figure 1.7: Upper: Schematic illustration of hyperbranched polymer linker and the formation of self-assembly of gold nanoparticles into the core-satellite nanostructure. Lower left: TEMicrograph of core-satellite nanostructure at the satellite to core ratio of 10. Scale bar: $50 \mathrm{~nm}$. Lower right: SERS intensity increases with the number of the number of satellites per core NP. Adapted with permission from ref. [40]. Copyright 2014 Royale Society of Chemistry. 
Our group has established a method to prepare gold-planet-goldsatellite nanostructure by using 4-arm star poly(N PAAM) with aurophilic trithiocarbonate groups on their chain termini.[41] This approach utilizes multiple advantages of controlled radical polymerization techniques. The topology ("4-arm") of linker polymer is easily determined by using a 4-arm star RAFT (reversible addition-fragmentation chain transfer) agents. The 4arm RAFT polymer can be effectively attached to $\sim 13 \mathrm{~nm}$ citrate-capped AuNPs using a grafting-to approach in colloid with free (not surface-bound) arms ready for catching satellite nanoparticles, as evidenced by STEM darkfield image (Figure 1.8) ${ }^{[42]}$ and simulations ${ }^{[43]}$. Adding an excess of satellite $\sim 5 \mathrm{~nm}$ AuNPs (Brust-Schiffrin type) will induce the self-assembly process creating a well-defined planet-satellite nanostructure while excess satellite nanoparticles can be removed by centrifugation. Compared with the hyperbranched polymer, the star polymer has well-defined topology and size. This character enables full control over the arrangement of the satellite nanocomponents (Figure 1.9). Statistically, the average distance between planet AuNPs and satellite AuNPs can be tuned between 3 to $20 \mathrm{~nm}$ by varying the molecular weight of the linker polymer from 12 to $62 \mathrm{~kg} / \mathrm{mol}$. 

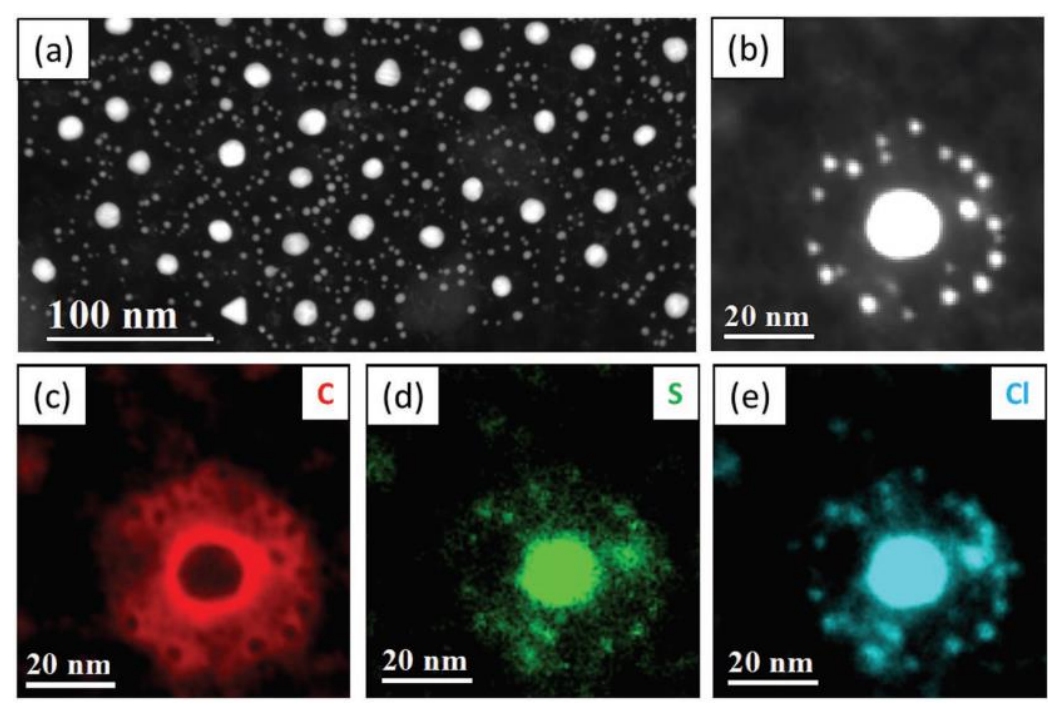

Figure 1.8: a,b)STEM dark-field image of the gold planet-satellite nanostructures. c-e) Elemental mapping of the elements carbon (red), sulfur (green), and chlorine (blue). Adapted with permission from ref. [42]. Copyright 2016 Wiley-VCH Verlag.

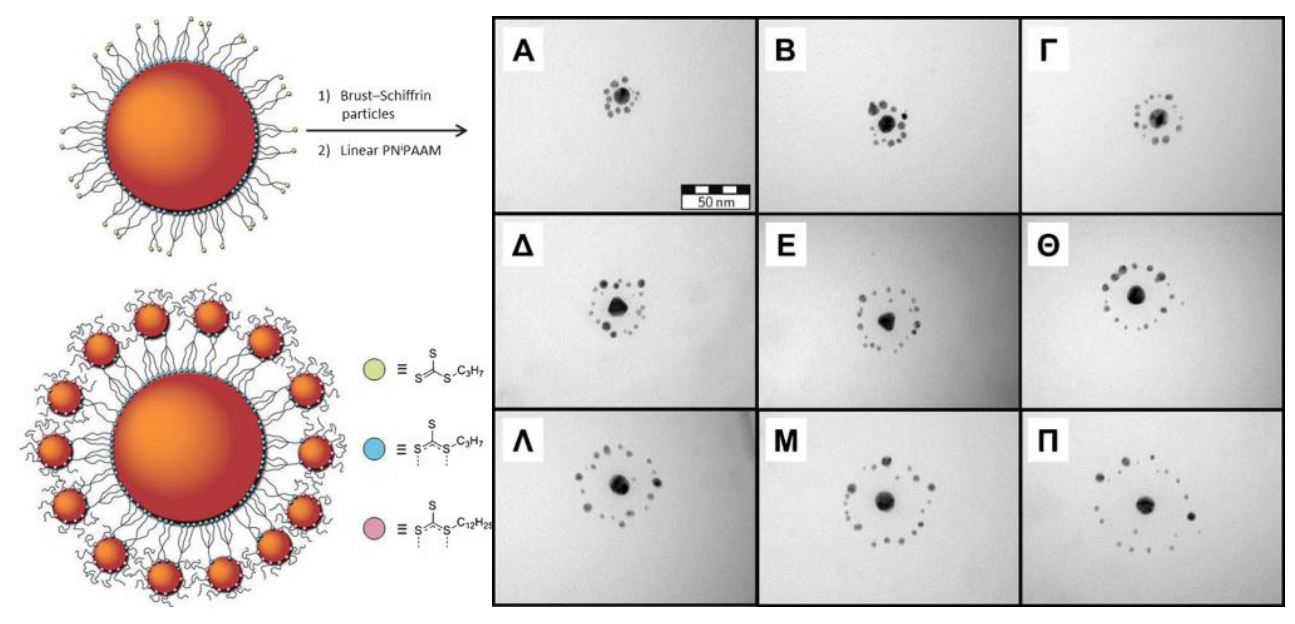

Figure 1.9: Left) Synthetic scheme of the planet-satellite nanostructures. Right) TE micrographs of the planet-satellite nanostructures. Adapted with permission from ref. [41]. Copyright 2014 Wiley-VCH Verlag.

Sulfur terminated polymer with sophisticated topological designs showcases the effective and flexible ability for self-assembly of noble-metal nanoparticles. However, for nanoparticles with a more distinct nature such as silica, another anchoring technique is inevitably required. 
As one of the most intensively studied and widely used nanomaterial, silica nanoparticles possess well-known surface chemistry owing to its rich surface - $\mathrm{OH}$ groups in a density of $5 \mathrm{OH} / \mathrm{nm}^{2}{ }^{2}{ }^{[44]}$ Using silane anchor moiety, small molecules can be easily introduced onto the silica surface with high capping density. It is worth mentioning that introducing larger molecules, i.e., synthetic or biological macromolecules onto silica directly by using silane chemistry ("grafting-to"), is less favored than first modifying surface with the small functional group which can be used for further functionalization. ${ }^{[45]}$ Based on the convenient silane chemistry, RAFT agents can be introduced on to the surface using a simple 2-steps protocol.[46] The details of the methods for introducing RAFT agents and the following polymerization from the surface of silica nanoparticles will be discussed in section 2.4.

If the surface-induced polymerization is carried out under a so-called Rapproach (section 2.4.2), the RAFT group will stay at the outer termini making the polymer capped silica nanoparticle suitable for attaching satellite nanoparticles. Böcker et al. have reported a method to fabricate AuNPs decorated silica-poly(NiPAAM) nanostructure (Figure 1.10).[47] The nanoassemblies showed a 3D structure in TEM rather than a 2D distribution as Rossner's work, probably due to the large size of silica as the planet ( $\sim 200 \mathrm{~nm}$ ). Owing to the stimuli-responsive property of poly(NiPAAM), the core-satellite nanostructure can easily succeed these favored properties from the polymer component making the hybrid nanostructure capable of potential sensor applications. ${ }^{[47-49]}$ 


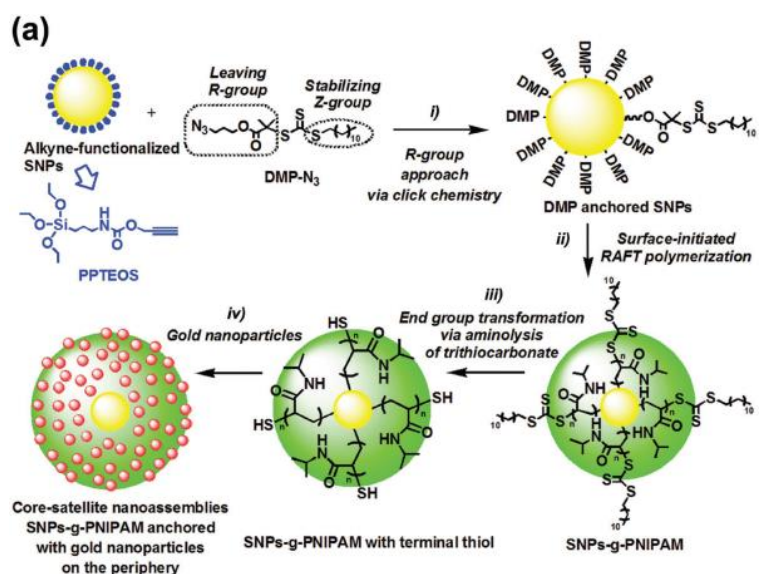

(b)
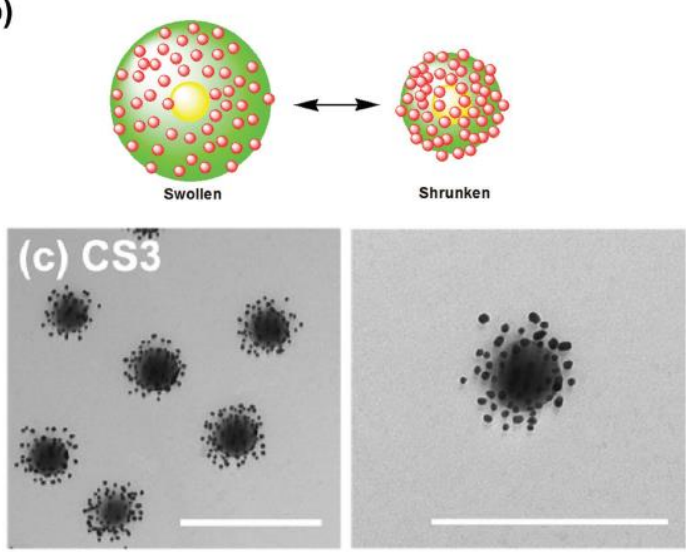

Figure 1.10: a) Synthetic routine to fabricate the core-satellite nanoassemblies. The polymer capped silica is fabricated by first introducing RAFT agent to silica surface via 2-steps click chemistry and successive surface-induced RAFT polymerization with NiPAAM. b) Illustration of the thermo-responsive ability of core-satellite nanostructure due to the usage of poly(NiPAAM). c) TEMicrograph of core-satellite nanostructure, scale bar is $500 \mathrm{~nm}$. Adapted with permission from ref. [47]. Copyright 2017 Wiley-VCH Verlag.

\subsection{Conclusions}

We have seen in section 1.2 the high efficiency and versatile strategy for fabricating core-satellite nanostructure with the small molecular linker. The distance between core and satellite nanocomponents was limited in very close range causing a strong interaction between nanoparticles. These 
structures were found to be very suitable candidates for applications like surface-enhanced Raman experiment, in which the close interparticle distance is favored. It is worth noting that coating the core nanoparticles with a well-defined silica shell is a useful approach for precisely creating spacing between core and satellite nano-components. The numerous linking strategies using molecular linker can also be built into polymeric linker by introducing the corresponding functional moieties (e.g., as end-group) to the polymer structure.

The development of the DNA based planet-satellite nanoassemblies has been resulting in a new explosion of interest in medical research. However, the actual clinical application of these nanoassemblies is hampered by the infeasibility in the large-scaled production. The intrinsic properties from DNA give many intriguing applications for the nanoassemblies (e.g., RNA detection). The well-defined structure of the DNA linker enables the encoding of interparticle distance which can be used to tune the interaction between core and satellite nanoparticles.

Insofar, functional polymeric linker synthesized by controlled polymerization provides several advantages from its precise macromolecular architecture. The interparticle distance can be easily monitored by tailoring the chain length of linker polymer. The spacing is an important parameter to tune the interaction between core and satellite nanoparticles and thus to optimize the performance of nanostructures specifically in the realm of surface plasmon enhanced coupled and controlled fluorescence since the metallic surface-induced fluorophore quenching occurs at a short distance $(<5 \mathrm{~nm}){ }^{[50]}$ It is reported that the most efficient range for AuNP induced fluorescence enhancement is $30 \sim 35 \mathrm{~nm}$, i.e., a spacer with well-defined thickness between AuNPs and fluorophore plays the key role to optimize the fluorescent ability.[51] Fluorescence has become the dominant detection/sensing technology in medical diagnostics and biotechnology. Building a planet-satellite nanostructure could be 
envisioned as one of the most efficient strategies for colloidal applications on this topic. Furthermore, the well-studied functional polymers can easily introduce the further unique abilities to the nanostructure e.g., temperature-response, ${ }^{[52,53]} \mathrm{pH}$-response, ${ }^{[54,55]}$ and sensing ${ }^{[56,57]}$. 


\section{Chapter 2}

\section{Designing Binary Nanostructure: Methods and Building Blocks}

\subsection{Preface}

The hierarchical self-assembly of distinct nanoparticles into ordered nanostructure requires a legitimate choice of building material. In this regard, a straightforward and efficient chemistry approach is highly favored to initiate the self-assembly process. The surface modification on the nanoparticles requires first a sufficient surface activity on the nanoparticle: strongly bond surfactant on nanoparticles often jeopardize the ligand exchange process especially for macromolecules. This chapter provides a detailed insight into the design of the fabrication strategy for the optimal production of binary nanostructures used in this thesis. The detailed properties of each type of nanocomponents will be reviewed specifically. Focus will be laid on the surface activity and surface chemistry of each nanoparticles which plays a vital role for the success of the self-assembly process.

In this thesis, two types of AuNPs (produced with the citrate reduction and two-phase Brust-Schiffrin method), oleylamine capped AgNPs, and silica nanoparticles are used for the fabrication of gold-planet-silversatellite and silica-planet-gold-satellite nanoparticles. All the nanoparticles possess very different surface activity and dispersibility in a different solvent. These properties will be discussed in section 2.2 and 2.3.

Section 2.4 will review the method to create dense polymer brush capped AuNPs and $\mathrm{SiO}_{2}$-NPs which are served as planet nanocomponents for two different projects in this thesis. Due to their distinct chemical nature, they are functionalized with dense polymer shells via different strategies. 
For Au planet, star RAFT polymer shell will be introduced to the surface of citrate-capped AuNPs by a grafting-to approach; for $\mathrm{SiO}_{2}$ planet, surfaceinitiated RAFT polymerization will be applied.

\section{$2.2 \quad$ Noble metal nanoparticles}

All the noble nanoparticles colloids used in this thesis were freshly prepared. The detailed experimental procedure can be found in Chapter 7.

\subsubsection{Gold nanoparticles}

$\sim 13 \mathrm{~nm}$ citrate-capped AuNPs will be used as the planet nanoparticles for gold-planet-silver-satellite nanostructures and as the satellite nanoparticles for silica-planet-gold-satellite nanostructures. The formation of AuNPs is proceeded by the reduction of $\mathrm{Au}^{3+}$ from $\mathrm{HAuCl}_{4}$ with citrate in refluxing water. ${ }^{[58]}$ The $\sim 13 \mathrm{~nm}$ size (Figure 2.1) of AuNPs is ideal for the investigation of plasmonic phenomena: the narrow absorption SPR (surface plasmonic resonance) band at $\sim 520 \mathrm{~nm}$ gives the red color of the AuNPs sol. This type of AuNPs is electrostatically stabilized [59] by citrate anion and has long-term colloidal stability (over the years).

Due to the weak interaction and low capping density of citrate ions on the surface, the citrate-capped AuNPs is highly active towards aurophilic ligand, providing easy access for ligand exchange e.g., with sulfur-containing molecules like polymer,[17] DNA ${ }^{[60]}$ and peptide. ${ }^{[61]}$ The high surface activity makes this type of AuNPs susceptible to impurity (e.g., heavy metal ions, change of $\mathrm{pH}$ value) which influents the ion equilibrium. The concentration of as-synthesized AuNPs is approximately $0.1 \mathrm{mg} / \mathrm{ml}$ and cannot be variated much due to the concentration-controlled kinetics of particle growth. Typically, this reaction is carried out in a $100 \mathrm{ml}$ batch.[17] In this work, the synthesis can be up-scaled to $500 \mathrm{ml}$ without influence on the size distribution. 
Since the citrate-capped AuNPs are stabilized by electrostatic interaction, the direct exchange of solvent into another solvent without any surface modification of AuNPs could be awkward. Adding organic solvent (e.g., acetone, THF) into aqueous colloid often causes instant aggregation of AuNPs. However, the citrate-capped AuNPs can be highly diluted with p. a. grade ethanol (99.8\%) without any aggregation.

The second type of AuNPs (so-called Brust-Schiffrin type[62]) used in this work is the tetrabutylammonium bromide (TOAB) capped $\sim 4 \mathrm{~nm}$ AuNPs, which are synthesized from a two-phase reduction of the gold salt precursor with $\mathrm{NaBH}_{4}$. The as-synthesized AuNPs are dispersed in toluene with weakly bonded TOAB on the surface. The TOAB ligand can also be easily replaced by aurophilic ligand like RAFT polymer. The $\sim 4 \mathrm{~nm}$ AuNPs possess a much weaker SPR band than the $\sim 13 \mathrm{~nm}$ AuNPs. It is worth noting that due to the small size of $\sim 4 \mathrm{~nm}$ AuNPs, they cannot be gathered using centrifugation.

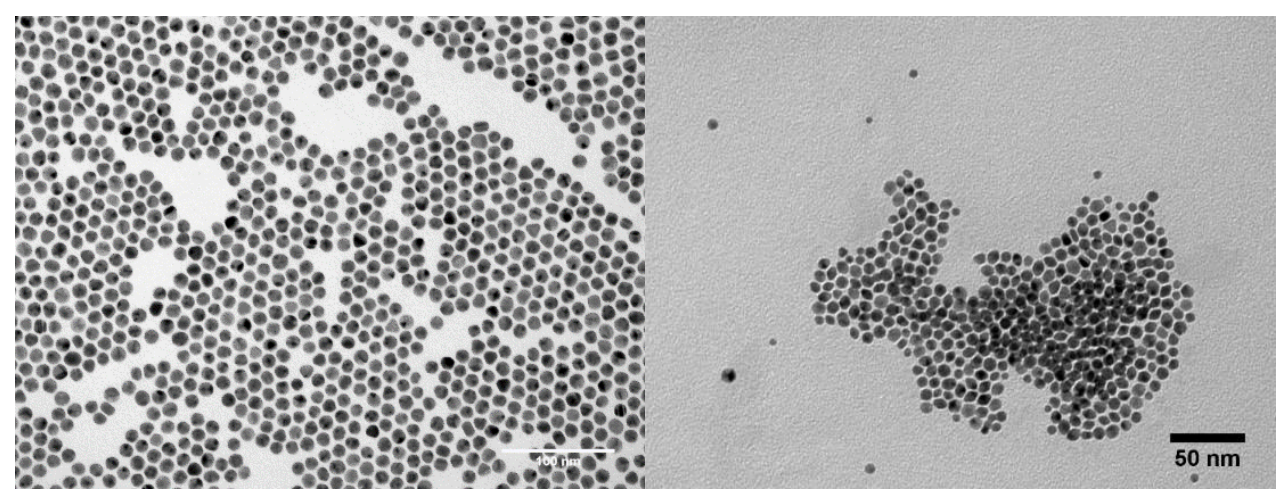

Figure 2.1: Exemplary TEM images of $\sim 13 \mathrm{~nm}$ AuNPs (left) and $\sim 4 \mathrm{~nm}$ AuNPs (right). The scale bars are $100 \mathrm{~nm}$ and $50 \mathrm{~nm}$, respectively. The size distribution of these NPs is charted in section 7.3.

\subsubsection{Silver nanoparticles}

Silver nanoparticles are notorious for their susceptibility to oxidation; thus, they must be prepared under the inert gas atmosphere and used instantly to avoid oxidation. Considering the dispersibility and overall yield, 
$\sim 9 \mathrm{~nm}$ oleylamine (OA) capped AgNPs are chosen as satellite nanoelement for gold-planet-silver-satellite nanoassemblies. The strong SPR band at $\sim 400 \mathrm{~nm}$ gives AgNPs an intense yellow color. The AgNPs are formed by a reduction of $\mathrm{AgNO}_{3}$ with oleylamine in refluxing toluene (Figure 2.2). The oleylamine serves as precursor ligand for dissolving $\mathrm{AgNO}_{3}$ in toluene, reduction agent and capping ligand for AgNPs. The large excess of oleylamine must be removed for further functionalization. However, if the removal of oleylamine is overdone, an insufficient capping density of OA on AgNPs quickly causes aggregation. Tuning the capping density of OA on AgNPs for the suitable surface activity while possessing sufficient colloidal stability is the key for the successful ligand exchange with polymer and fabrication of the planet-satellite nanostructure. The semi-quantitative monitoring of OA capping density on AgNPs is achieved in this thesis by using segmentation/centrifugation cycles under special conditions. The insight of surface activity of OA capped AgNPs in correlation with OA content will be discussed in detail in section 4.2.

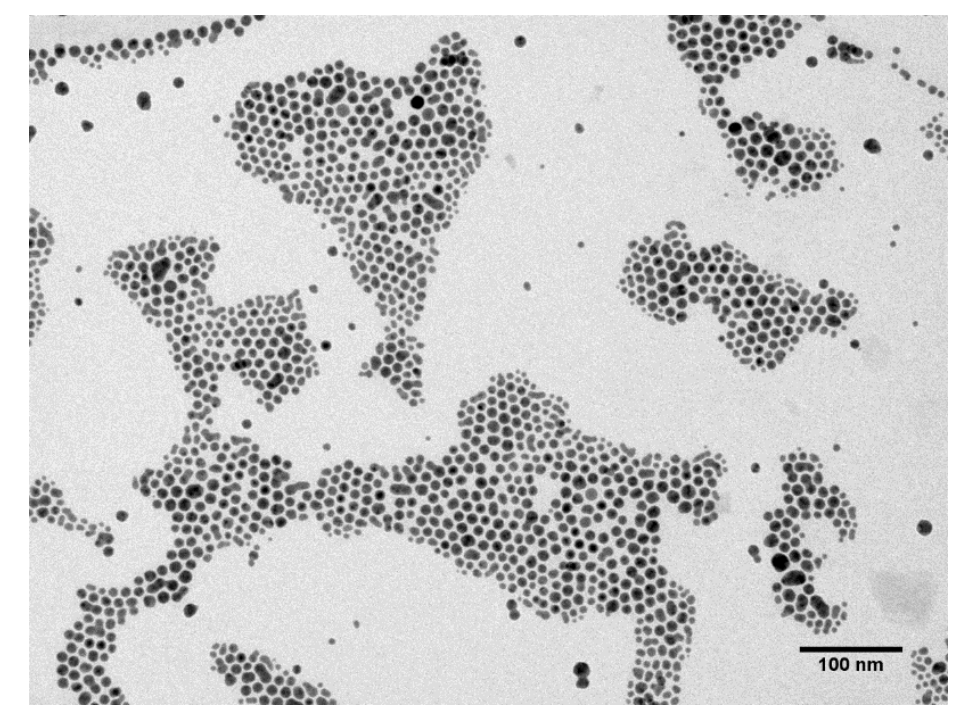

Figure 2.2: Exemplary TEM images of OA capped $\sim 9 \mathrm{~nm}$ AgNPs. The scale bar is $100 \mathrm{~nm}$. The size distribution of these NPs is charted in section 7.3. 


\subsection{Synthesis and functionalization of silica nanoparticles}

Silica nanoparticle ( $\left.\mathrm{SiO}_{2}-\mathrm{NP}\right)$ is chosen as the planet nano-unit for silicaplanet-gold-satellite nanostructure. This section focuses on the synthesis and general chemical modification on silica nanoparticles surface. The detailed experimental procedure can be found in Chapter 7 .

The silica nanoparticles are typically obtained by hydrolysis of tetraethyl orthosilicate (TEOS) in an aprotic solvent (e.g., ethanol, methanol) with ammonia as a catalyst. Conventional one-phase Stöber method ${ }^{[63]}$ by mixing ammonia and TEOS in alcoholic solution yields silica NPs with very limited roundness due to poor-defined nucleation and growth kinetics. In order to create silica nanoparticles with the highest roundness and a diameter of less than $50 \mathrm{~nm}$, the microemulsion technique ${ }^{[64-66]}$ is used here. The kinetic of $\mathrm{SiO}_{2}$-NP formation is controlled by creating nano-scaled $(\sim 20 \mathrm{~nm})$ water-inoil reverse micellar micro-reactors by using IGEPAL CO-520 surfactant in water/cyclohexane system. The hydrolysis reaction will proceed inside each micelle providing a well-defined and straightforward growing process of single $\mathrm{SiO}_{2}$-NP (Figure 2.3).

It is worth noting that using IGEPAL C0-520 based microemulsion technique with additional hydrophilic nanoparticles (e.g., OA capped quantum dot, ${ }^{[67]} \mathrm{OA}$ / oleic acid capped $\mathrm{Fe}_{3} \mathrm{O}_{4}{ }^{[64]}$ ) as seed for silica growth, a one-to-one coating of targeted nanoparticles can be archived. This flexible ability to introduce further nanoparticles in the planet nano-unit brings further favored functions like fluorescence and magnetic properties.

The surface of $\mathrm{SiO}_{2}$-NPs carries about 5.0 Si-OH groups / nm ${ }^{2},{ }^{[68]}$ which are ready for surface modification with alkoxysilanes for varies functions. In fact, the surface silane chemistry plays a central role for several different tasks in this thesis: first, effective anchoring of RAFT agent to the surface of silica is the keystone for introducing dense polymer shell onto silica surface in a well-defined fashion (section 2.4.2). In section 5.4, patterned AuNPs and 
$\mathrm{SiO}_{2}$-NPs are transferred from $\mathrm{CaF}_{2}$ onto the PEG matrix. The immobilization of transfer agent ${ }^{[69]}$ on $\mathrm{SiO}_{2}$-NPs is again provided by silane chemistry. The reaction condition of $\mathrm{SiO}_{2}$-NPs with alkoxyl silane is generally very flexible: the experiment condition can be varied according to the dispersibility of $\mathrm{SiO}_{2}$-NPs and solubility of alkoxyl silane to achieve the optimal performance of the reaction. The detailed experimental procedure of each silane reaction can be found in Chapter 7 .

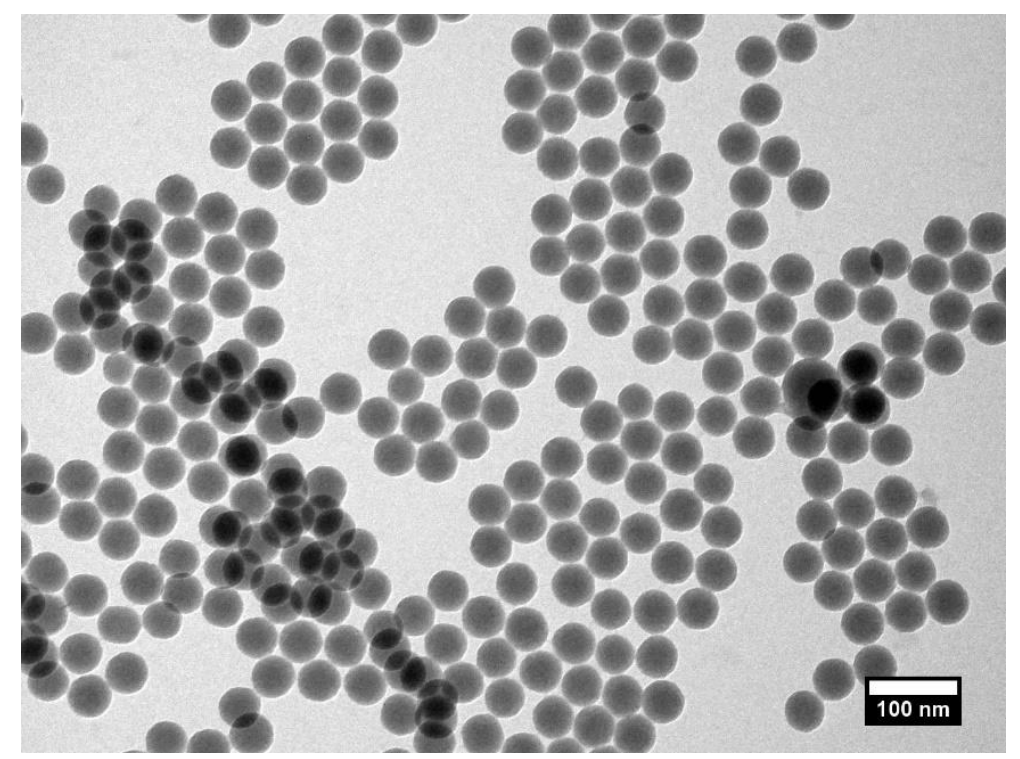

Figure 2.3: Exemplary TEM images of $\sim 40 \mathrm{~nm} \mathrm{SiO}_{2}$-NPs synthesized via w/o microemulsion method. The scale bar is $100 \mathrm{~nm}$. The size distribution of these NPs is charted in section 7.3.

\subsection{The approaches of anchoring polymer onto nanoparticles' surface}

In this section, the strategies to immobilize dense RAFT polymer onto AuNPs and silica NPs for the creation of the planet-nanocomponents are demonstrated in section 2.4.1 and 2.4.2, respectively. For AuNPs, the grafting-to approach with 4-arm star RAFT polymer will be employed, and 
for $\mathrm{SiO}_{2}$-NPs, surface-initiated RAFT polymerization (grafting-from approach) will be implemented.

\subsubsection{Star polymer capped AuNPs: grafting-to approach}

Using a colloidal self-assembly method, dense brush of RAFT polymer can be formed onto the surface of AuNPs (Figure 2.4).[17,41] First, the star RAFT polymer is synthesized ex situ using radical polymerization with trithiocarbonated[70] terminated star RAFT agents. ${ }^{[71]}$ Here, the 4 arm star RAFT agent serves as molecular weight and shape regulator. Owing to the controlled growth kinetics ${ }^{[72]}$ of each polymer chain (arm), the star polymer can maintain a narrow molecular weight distribution. The star RAFT polymer is designed to offer a controlled polymerization via R-group[73] approach making the RAFT end-group at the chain-end of the polymer. Poly$N$-isopropylacrylamide (poly(NiPAAM)) is chosen here for its general good solubility in a polar solvent (e.g., ethanol, chloroform, water).

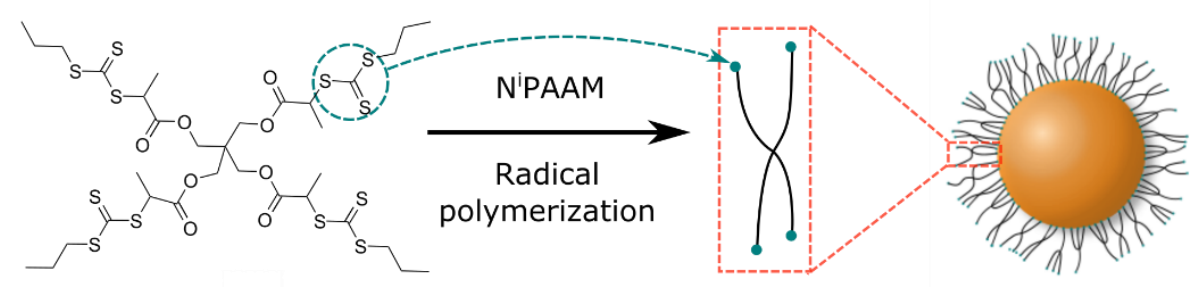

Figure 2.4: Synthetic scheme of star polymer capped AuNPs.

The as-synthesized RAFT poly(NiPAAM) has a strong affinity towards the gold surface. The self-assembly of RAFT polymer onto AuNP's surface can be achieved by merely mixing AuNPs colloid with RAFT polymer solution at room temperature. The excess of the polymer can be easily removed by centrifugation process. This method is very reproducible for both linear[17] and star polymer.[41,74] The star RAFT poly(NiPAAM) 
functionalized AuNPs possesses high capping density of polymer ${ }^{[42]}$ ( 0.2 to 0.4 chains per $\mathrm{nm}^{2}$, depends on the molecular weight of polymer). Once the nanostructure is casted on a carbon film by using e.g., drop-casting method, the dense polymer brush on the AuNPs enables a precise 2D hexagonal nanopatterning of the AuNPs with tunable distance from 5 to $40 \mathrm{~nm}$ by monitoring the chain length of polymer (Figure 2.5).

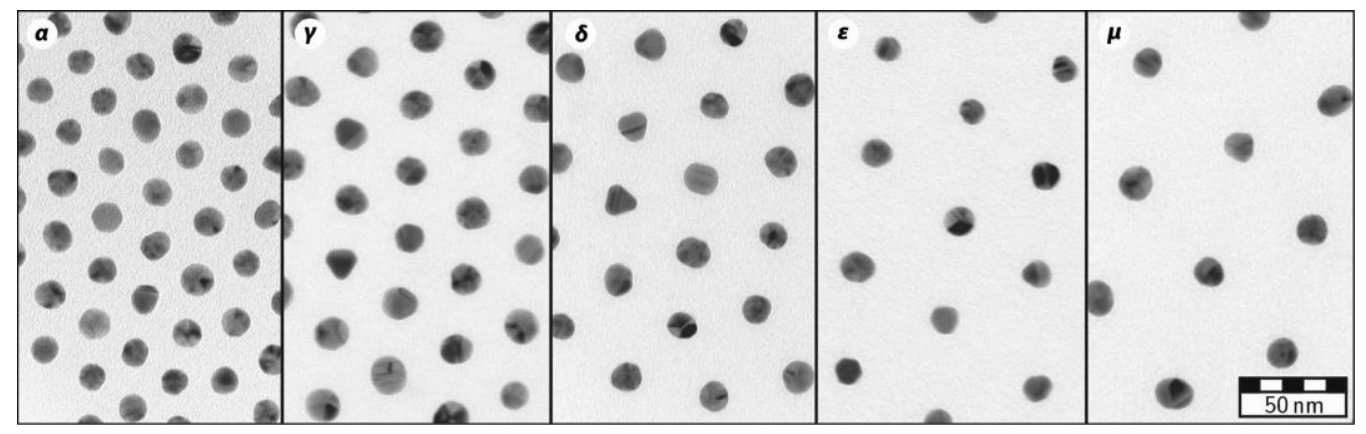

Figure 2.5: Hexagonal pattern of linear poly(NiPAAM) capped 13 nm AuNPs. The interparticle distance can be tuned by the chain length of the polymer brush. Adapted with permission from ref. [17]. Copyright 2013 American Chemical Society.

\subsubsection{Surface-induced RAFT polymerization from silica: grafting-from approach}

There are two general approaches to synthesize polymer-tethered silica nanoparticles: "grafting-to" and "grafting-from".[75] In the "grafting-to" approach, the functionalized polymer with the anchoring group is synthesized ex situand reacts with silica NPs in excess in a separate step. As the grafting reaction processes, the grafted polymer blocks the diffusive approach of the free polymer chain, typically resulting in low surface grafting density.

For the fabrication of high-density polymer brush capped silica NPs, the "grafting-from" approach often gives a better result by polymerization from surface-modified silica NPs. In this thesis, silica nanoparticles are first functionalized with the RAFT group [76] by using a two-step reaction (Figure 
2.6). The controlled surface-initiated polymerization is then carried out on the RAFT functionalized silica NPs: the monomers are added to the growing polymer brush under a controlled RAFT kinetic. This approach easily overcomes the limitation of grafting density in the "grafting-to" approach while keeping the grafted polymer chain with narrow molecular weight distribution. In practice, additional free RAFT agent is added to the polymerization's colloid which helps to dose the monomer-to-RAFT ratio and offers a secure prediction of the chain length.

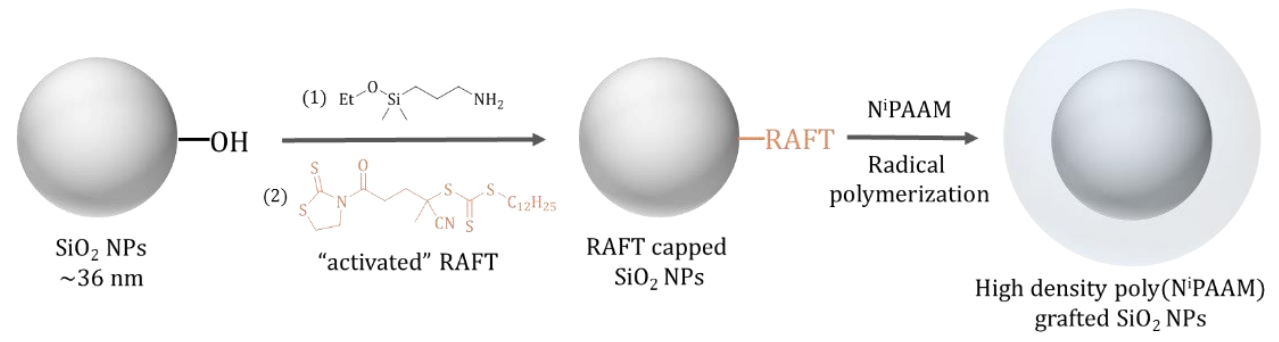

Figure 2.6: Synthetic scheme of poly(NiPAAM) capped silica NPs. In the first step, the silica is modified with APTES with the $\mathrm{NH}_{2}$ end group which further anchors with the mercaptothiazoline tailored RAFT agent ("activated RAFT"). The RAFT functionalized silica NPs polymerized with NiPAAM resulting in high-density poly(NiPAAM) brush grafted $\mathrm{SiO}_{2}$-NPs.

In order to create a well-defined planet-satellite nanostructure for binary nanoparticle patterning, the silica core must be wholly separated and individually polymerized. This requires a perfect dispersion of silica nanoparticles in the reaction's medium in each reaction's step. On this point, this thesis provides a series of optimized reaction's conditions for polymerization of NiPAAM and MMA (section 5.2.2).

\subsection{Conclusion}

Section 2.2 reviewed the synthesis and properties of $\sim 13 \mathrm{~nm}$ citratecapped AuNPs, $\sim 4 \mathrm{~nm}$ TOAB-capped AuNPs, and $\sim 9 \mathrm{~nm}$ oleylamine capped AgNPs. The surface activity and solvent environment for each type of 
nanoparticles should be considered for any functionalization or selfassembly process. Section 2.4.1 demonstrated a straightforward grafting-to approach to anchor high-density RAFT functional polymer onto the surface of AuNPs by its inherent trithiocarbonate end-group. The high grafting density of the polymer shell provides a precise 2D nanopatterning of AuNPs on the substrate with further control of interparticle distance in the range of 5 to $40 \mathrm{~nm}$ by tuning the chain length of grafted RAFT polymer. The idea of programming the graph of patterned nanoparticles by monitoring the size of the grafted polymer is the central strategy for this thesis.

Section 2.3 reviewed the synthesis of spherical silica nanoparticles using w/o reverse microemulsion technique and its general surface chemical modification using silane chemistry. The method yields silica nanoparticles with narrow size distribution with selectable diameter from $\sim 30$ to $70 \mathrm{~nm}$ and very high roundness. In order to build $\mathrm{SiO}_{2}$-NPs into well-defined planet-satellite nanostructure with tunable particle distance, high-density polymer brush must be introduced to the silica surface in a coordinated fashion with completely separated $\mathrm{SiO}_{2}$-NPs. To do this, silica nanoparticles was first decorated by RAFT agent using a two-step silica chemistry. Then the successive surface-induced radical polymerization (grafting-from) were proceed. The resulted dense polymer chain capped silica nanoparticle possesses RAFT moieties on its periphery which will be used for creating planet-satellite nanohybrid. 


\section{Chapter 3}

\section{Nanopatterning Mediated Cell-Responses}

\subsection{Preface}

The highly precise planet-satellite nanostructure with its programmable binary nanopattern opens up intriguing applications as a template for creating biomolecular patterning for biological research, especially in dermatology.

Cell membrane receptors are located on the surface of each cell; these biomolecules have the ability to sense and transmit chemical and physical signals and induce the corresponding cell response. Triggering the receptors using an artificial substrate with known nanopatterned bioactive molecules such as adhesion peptides growth factors is crucial in understanding the general and specific cell-material interactions, which can elucidate the mechanisms of cell behavior such as adhesion, migration, activation. ${ }^{[8,77-79]}$ For example, focal adhesion was intensively studied by using precisely arranged 2D gold nanoparticle patterns on the surface with biological moieties anchored on the particles. In these works, the template used to create nanopatterned bioactive molecules is a self-assembled monolayer of hexagonal patterned AuNPs by block copolymer micelle lithography (section 3.2.1). The spacing between AuNPs has been shown to have a dramatic impact on the cell-response. This precise interparticle distance dependent response is highly interesting and can be useful for optimizing therapeutic conditions for skin cancer.

However, the development of this approach has met its bottleneck. Due to the complexity of the biological environment, a binary biological patterning model with tunable spacing must be established to mimic the 
natural environment, thus gain insight into the cross-talking and synergy effect of distinct biological moieties in a detailed range-depended fashion.

However, the fabrication of a precisely ordered binary nanopatterned surface remains a challenge besides the high demand due to the various binary biological structural nature. As an example, integrin is a heterodimer (meaning it consists of two subunits) and the confirmation and distance of both subunits, which plays a vital role in focal adhesion. Artificially fabricating analogs binary structure with a predetermined and tunable pattern could easily open up a novel experimental design for the next generation research in this field.

This chapter will focus on the state-of-the-art of nanopatterning of noble metal nanoparticles and the review of nanopatterned nanostructure for cell response experiments, including a detailed discussion of the advantages which the silica-planet-gold-satellite nanostructure can offer.

\subsection{Nanopatterning: a brief state-of-the-art review}

Creating a patterned structure with distinct properties in the nanoscale $(<100 \mathrm{~nm})$ is one of the central missions for nanotechnology and heavily applied in various fields, e.g., fabrication of computer chips, energy harvesting, photonics. ${ }^{[80]}$ Breakthroughs in this field often lead to significant advancement of nanotechnology. The widest technique for nanopatterned manufacturing structure is nanolithography including optical,[80] electronbeam,[81,82] scanning probe, ${ }^{[83-86]}$ nanoimprint ${ }^{[87,88]}$ and nanoparticlebased[89-91] techniques. The resolution of state of the art electron-beam $(\sim 5$ $\mathrm{nm}{ }^{[92]}$ ) or scanning probe techniques (sub-50 $\mathrm{nm}^{[93]}$ ) is very high and they also have access for manufacture nanopatterning for bioactive molecules. ${ }^{[94-}$ 96] However, patterning a surface using a beam-based or scanning probe techniques often suffers from limited throughput to create a centimetersized sample (for typical cell-response experiment with sufficient statistical 
analysis[97]) in high resolution. ${ }^{[84,96]}$ The high time consumption and hardware cost also limit the accessibility of the high-end beam based nanolithography.

In this thesis, the nanopatterning is manufactured using a dip-coating technique to form a monolayer of polymer capped nanostructure on the substrate. Previous work ${ }^{[8,78,90,98]}$ has demonstrated that under optimized dip-coating conditions, AuNPs loaded diblock copolymer micelles can selfassembled into a homogenous monolayer forming a precise hexagonal pattern of AuNPs with tunable distance in sub-100 nm-scale. Section 3.2.1 will review the technique, advantages, and disadvantages of the block copolymer micelle nanolithography in detail.

Beside nanoparticle loaded block copolymer micelle, the RAFT polymer capped nanoparticles also show a highly ordered hexagonal self-assembled pattern once drop-casted onto a carbon-film (substrate for TEM).[17,99] Owing to the narrow size distribution of the capping polymer, the flexible nanostructures offer full control over size and interparticle distance of the hexagonal nanopattern. However, the drop-casting method only offers a nanopattern in a random $\sim 100 \mathrm{~nm}$ range, which limits the application of this approach.

This thesis introduces the dip-coating technique to fabricate monolayer of hexagonal patterned nanostructures, including simple linear capped AuNPs, silica NPs and the binary silica-planet-gold-satellite nanostructures. This allows a precise preprogramming of nanopattern presentation through the molecular design of the polymer component. The detailed study on the formation of monolayer will be discussed in section 5.4 . 


\subsubsection{Block copolymer micelle nanolithography for cell response experiments}

The basis of block copolymer micelle nanolithography is the selfassembly of block copolymer micelle carrying metal nanoparticles (e.g., AuNPs) into a uniform mono micellar layer on a solid substrate. ${ }^{[98,100]}$ The molecular weight of the block copolymer has a direct impact on the interparticle distance between the AuNPs. The patterning length of the block copolymer micelle nanolithography can be controlled between 30 and 200 $\mathrm{nm}$ by tuning the size of the block copolymer and the speed of dip-coating. The general limit of the patterning scale is the critical size of the stable polymer micelle. Due to this issue, the block copolymer micelle approach has difficulties reaching sub $30 \mathrm{~nm}$ resolution.

In these approaches, the AuNPs are obtained by reduction of precursor $\left(\mathrm{HAuCl}_{4}\right)$ that is loaded into the block copolymer inverse w/o micelle with $\mathrm{N}_{2} \mathrm{H}_{4}{ }^{[100]}$ The amount of $\mathrm{HAuCl}_{4}$ loaded in the micelle offers control over the size of the yielded AuNPs. Since the application of these nanopatterns is mainly focused on the bioactive molecules bonded to the surface of AuNPs, the size of AuNPs was intentionally chosen to be in the typically $5 \sim 10 \mathrm{~nm}$ range.[69,77,101] 

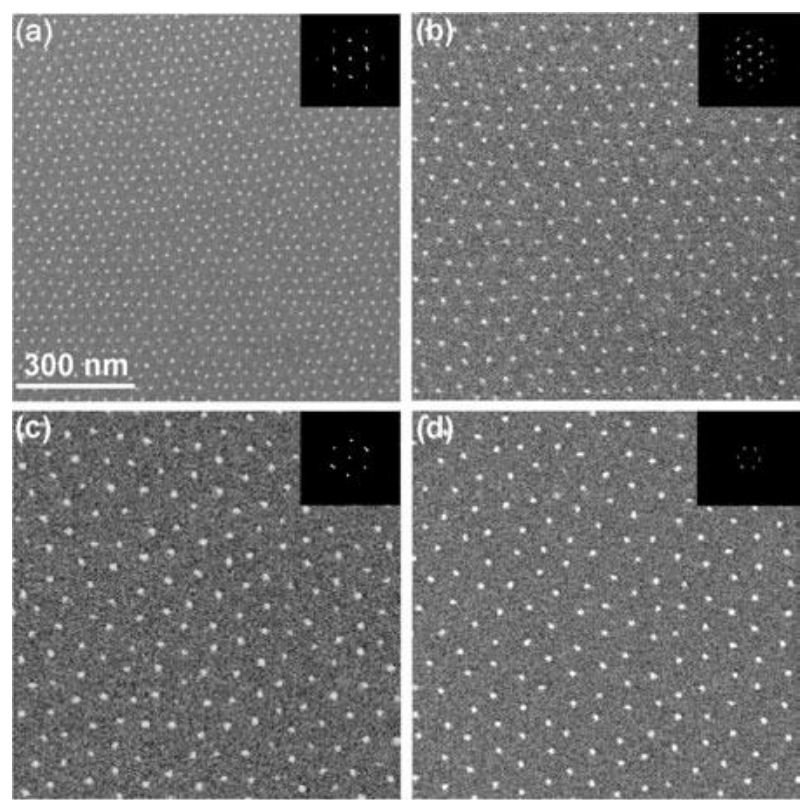

e

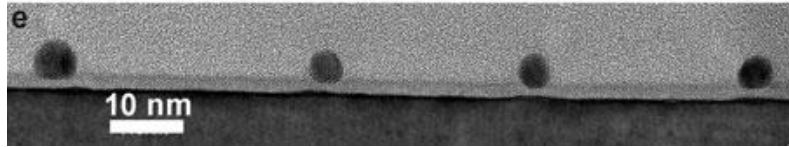

Figure 3.1: SEM micrograph of Si-wafers structured with AuNPs created by block copolymer lithography. The interparticle distance was varied from $28 \mathrm{~nm}$ to $85 \mathrm{~nm}$ by tuning the chain length of the block copolymer. Adapted with permission from ref. [98]. Copyright 2013 IOP Publishing.

The formation of a uniform monomicellar film on an inorganic substrate (e.g., glass, silicon wafer) is the critical step of utilizing colloid nanostructure for 2D nanopatterning. Standard methods, i.e., spin-coating and dip-coating, can be both applied here.[98] The uniform size distribution of block copolymer (from ionic polymerization) enables precise control over interparticle distance in the nanopattern (Figure 3.1). To enable the biological labeling of AuNPs, the micelle should be completely removed in order to expose the pure AuNPs to the environment without any residual contaminations. Using plasma (hydrogen, oxygen, argon) treatment under suitable conditions to remove a thin layer of polymer from the substrate thoroughly has been reported to be a very efficient and reliable method[102] and has been widely applied for creating nanoparticle patterned surface for 
cell response experiments. After plasma treatment, the surface of AuNPs is then successively anchored with thiolated biofunctional molecules such as peptides (e.g., RGD- and HAV-sequence), proteins or antibodies. These biological labels have a general strong affinity towards inorganic substrates like glass or silicon wafer. The cells also have the general intention to attach to the surface of the substrate. Those adverse effects must be strictly suppressed by sufficient surface passivation (PEGylation) and suitable experimental condition for biological labeling (e.g., concentration, $\mathrm{pH}$, incubation time, temperature).

The nanopatterned substrate has been served as a powerful experimental platform for cell response experiments. ${ }^{[78,79,97]}$ As an example, Figure 3.2 demonstrates the cell adhesion towards the RGD peptide patterned glass surface. ${ }^{[97]}$ In this work, osteoblast cells were incubated with a substrate hexagonally nanopatterned with RGD peptide (on AuNPs) with different spacing from 28 to $85 \mathrm{~nm}$. The cells have shown strong adhesion on the surface patterned with RGD with spacing below $58 \mathrm{~nm}$ and no adhesion behavior on those with a distance larger than $73 \mathrm{~nm}$. The drastic transition of the cell's behavior from $<58 \mathrm{~nm}$ to $>73 \mathrm{~nm}$ patterned surface suggests that $58-73 \mathrm{~nm}$ is a universal length scale for integrin clustering and activation. 

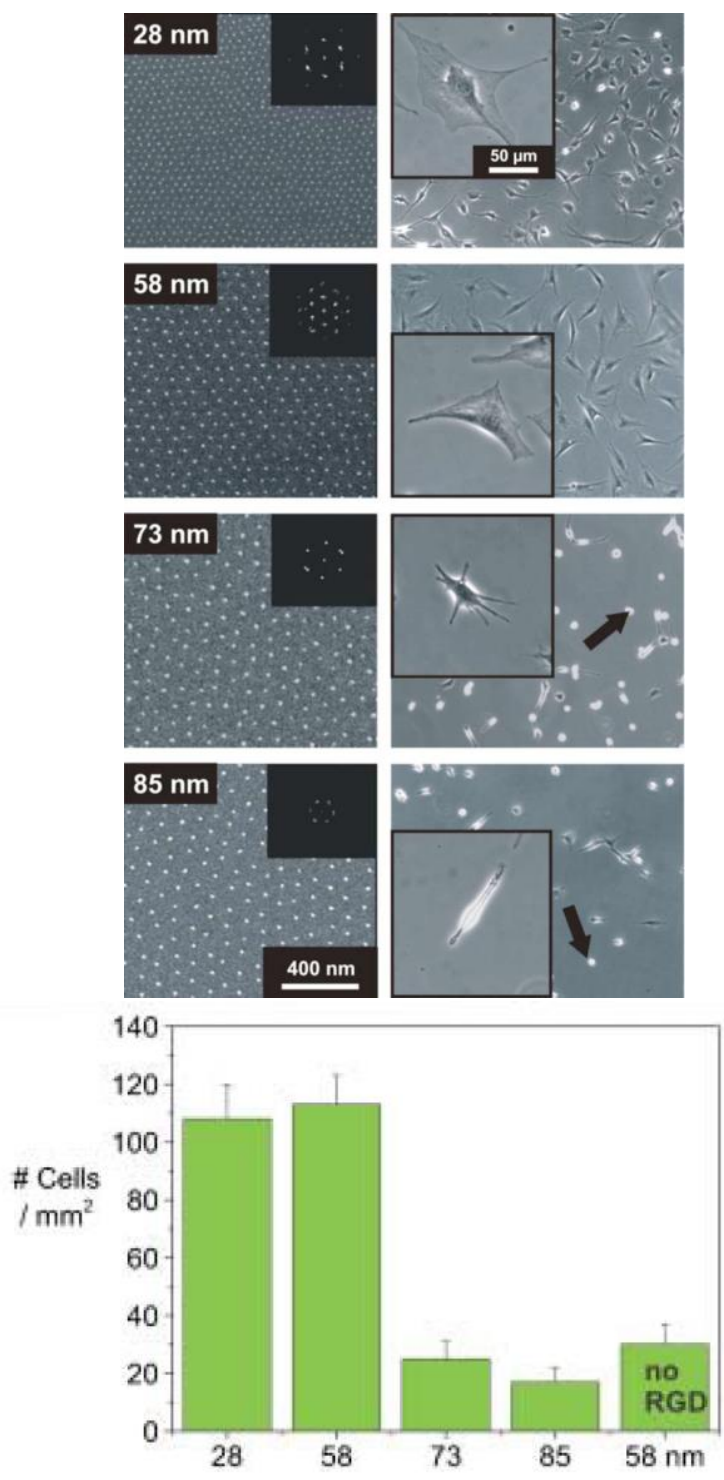

Figure 3.2: The spacing between hexagonal arrays of gold nanoparticles functionalized with RGD affects cell spreading and focal adhesion formation. The arrows indicate the nonadherent cells. Adapted with permission from ref. [97]. Copyright 2004 Wiley-VCH Verlag GmbH \& Co. KGaA.

In this context, the dip-coating method offers the advantage of creating a dipping-line, which is very handy for the cell-response experiments by comparing cell behavior on both sides of the dipping line (probe vs. 
background) on the same sample substrate. This dipping line offers very convenient and reliable control of the specific and exclusive biological functionalization on the AuNPs and sufficient passivation on the substrate surface simultaneously.

As a substrate for the cell-response experiments, glass has several inherent disadvantages for its inorganic nature. Beyond the surface passivation issue mentioned above, the mechanical properties of glass do not match the biological environment for the cells. A more advanced approach is to transfer the patterned nanoparticles onto PEG hydrogel[103] taking advantage of its biocompatibility, nontoxicity, and versatility of mechanical properties. Since the mechanical properties of the substrate also have an impact on cell behavior ${ }^{[104]}$ it is highly favored to tune the mechanical properties of the supporting matrix similar to the biological surface on which the biological affair is supposed to happen. By changing the monomer size and water content of the hydrogel, the stiffness of PEG hydrogel can sufficiently cover the range of the mechanical properties of all tissues.[105] To transfer the patterned nanoparticle from the inorganic surface onto PEG hydrogel,[106] a suitable acryl-linker must be introduced onto the surface covalently prior to the polymerization of PEG diacrylate (forming of hydrogel). The linker plays a pivotal role in transferring the nanoparticles and sufficiently binds them to the hydrogel matrix. With the absence of the nanoparticles, templates without AuNPs but only imprint (holes) will be pictured on the hydrogel (Figure 3.3). 


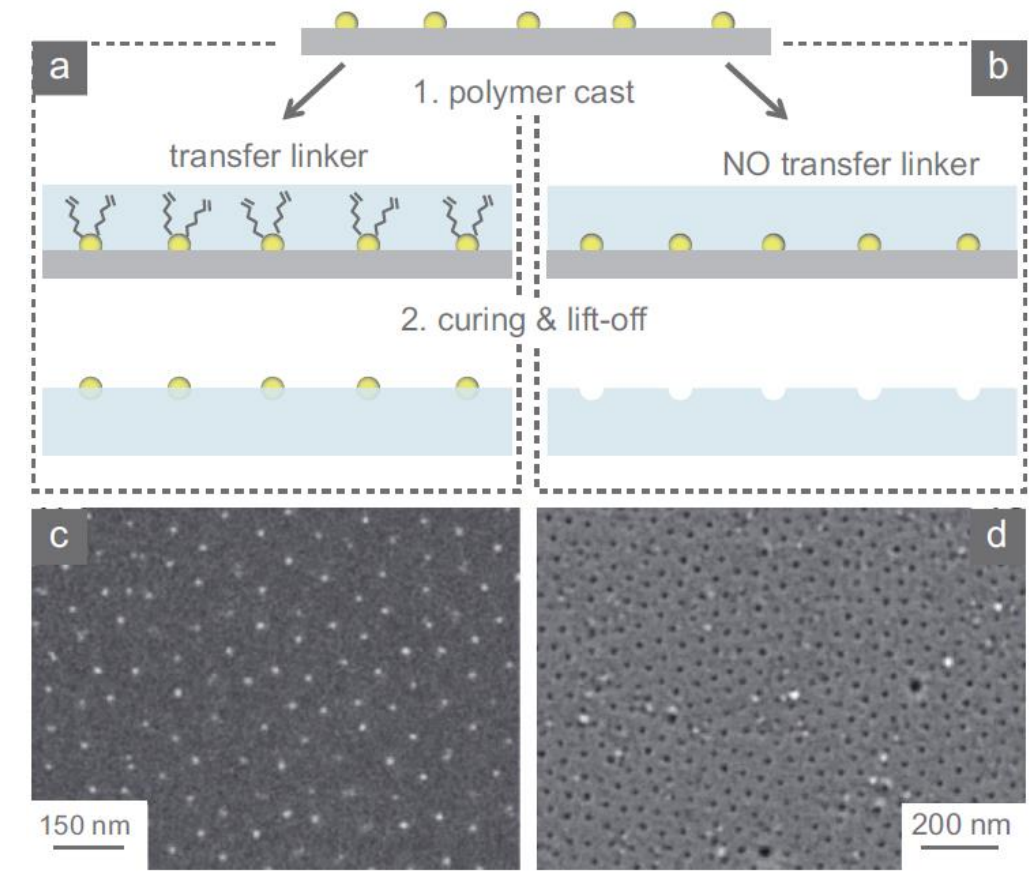

Figure 3.3: a) AuNPs can be transferred to a PEG matrix (c) by using a suitable transfer linker. The linker is covalently bound to the surface of AuNPs and cross-linked into the polymer. b) In the absence of a linker, only a porous imprint can be generated. Adapted with permission from ref. [107]. Copyright American Vacuum Society in 2011.

Developing a binary nanopatterned structure is of great interest for the next step of artificially induced cell response experiments due to the numerous biological affairs based on binary biological nature. As an example, integrin is a heterodimer (meaning it consists of two subunits) and the conformation and distance of both subunits, which plays a vital role in focal adhesion. In earlier work, ${ }^{[101]}$ the second bio-label is homogeneously distributed on the background while the first bio-label is anchored on the hexagonal patterned AuNPs. This approach lacks the control of the relation between both bio-labels and provides very limited information on the crosstalking mechanisms between two bio-labels. Recently, Spatz et al. have reported a binary nanopattern of $\mathrm{Au}$ and $\mathrm{TiO}_{2}$ nanoparticles on a PEG substrate for initiating cellular responses based on block copolymer micellar lithography (Figure 3.4).[69] In this work, both AuNPs and $\mathrm{TiO}_{2}-\mathrm{NPs}$ 
are independently arranged on the substrate (hydrogel). A controllable correlation with the defined organization between both nanoparticles was still missing in this approach.
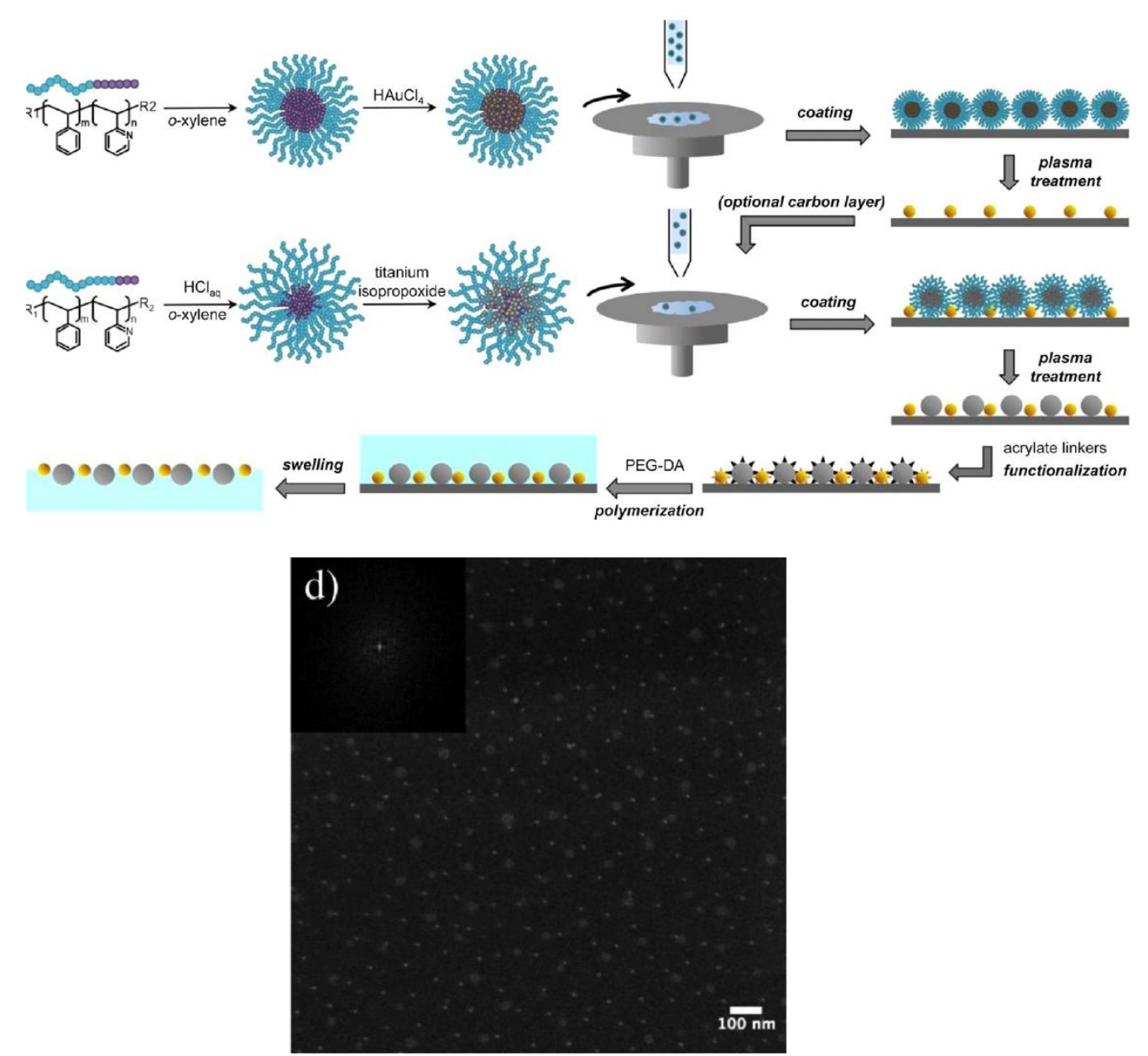

Figure 3.4: Preparation of binary patterned AuNPs and $\mathrm{TiO}_{2}$ NPs on Hydrogel. Lower SEMicrograph shows the distribution of both types of nanoparticles: the spacing between each type of NPs can be roughly controlled while the correlation between two nanoparticles is totally random. Adapted with permission from ref. [69]. Copyright American Chemical Society 2016.

The fabrication of a precisely ordered binary nanopatterned surface remains a challenge. In our project, the binary planet-satellites nanostructures provide a distinct pattern with the tunable distance between 
both nanoparticles, which gives access to a much more systematic and detailed study on cellular responses on binary biological stimulation. This knowledge gives insight into the unrevealed cross-talk mechanisms between distinct biological molecules. The primary requirements and challenges of creating such a sample for the biological experiments will be discussed in more detail in section 3.2.2.

\subsubsection{Challenges to fabricate binary planet-satellite nanopatterning for biological experiment}

From a stable colloid of the planet-satellite nanostructure to a binary bio-labeled substrate/matrix, a practical chemical approach, sophisticated experimental design, and careful handling are required. Biological and medical research always uses a large sampling size which requires a reliable and valid (both time and material) production of probes.

The choice of substrate for monolayer coating is crucial since further processing and functionalization require specific conditions; several limitations occur for the choice of the substrate, which must be considered. First of all, the substrate must be chemically completely distinguishable from gold and silica; otherwise, the specific binding on nanoparticles cannot be archived. Since silica and gold nanoparticles are chosen as a template and the bio-label will be introduced using thiol and silane moieties, respectively. In this regard, the substrate should contain no $\mathrm{OH}$ group (silane reaction) or thiolphile atom (e.g., heavy metal). Glass, metal oxide and metal substrate are not suitable to be used as a substrate for their similarity to the nanoparticles. The functionalized surface will be treated with plasma to completely remove polymer content to expose the surface of NPs for further functionalization. The substrate should survive plasma treatment; the organic-based material, e.g., polymer, is then not qualified for these criteria. 
Furthermore, the cell experiment is conducted in cell suspension, and the biological functionalization is also carried out in the water. The dip-coating must proceed in a relatively fast evaporating organic solvent: the substrate must be robust against both water and organic solvent. The live reaction of cells will be recorded with an optical microscope, and the substrate must be highly transparent in the visible spectrum. Not only the toxicity of the substrate must also be strictly controlled, but the affinity of the cell onto the surface, including the unspecific binding of bio-label towards substrate, must also be suppressed entirely or be passivated by somehow sufficient PEGylation. It is also worth mentioning that the substrate for coating should be as flat as possible to maintain the nanostructure in high-fidelity. To this consideration, crystal surfaces have decent advantages for creating a homogenous monolayer of nanoassemblies.

To sum it up, the required material must be transparent, nano-flat, nontoxic, nonreactive toward cell and biological label, tolerant towards the water and organic solvent, have access for sufficient PEGylation, to be differentiable from with gold and silica and stable for plasma treatment. Furthermore, the substrate should be affordable for large-scaled biological experiments.

There are only a few materials that match all these limitations. $\mathrm{CaF}_{2}$ was chosen here as the archetype substrate, which meets both the physical and chemical requirements. The most challenging here is the passive the surface for sufficient biological inertness, although $\mathrm{CaF}_{2}$ possesses no active chemical group (e.g., OH group) which induces unspecific cell or protein (peptide) attachment. The biological behavior of the substrate must be studied and the strategy for passivation must be tested with cell experiments.

A promising alternative for the substrate is to first dip-coat on the substrate (e.g., $\mathrm{CaF}_{2}$ ) and then transfer the nanoparticles onto PEG hydrogel for the biologic experiments. This approach brings several advantages i.e. no 
issues to the background, adjustable mechanical properties of the hydrogel, lower cost by reusing the expensive inorganic substrate. However, a suitable linker including the functionalization's condition for silica NPs must be optimized to achieve the effective transfer of nanoparticles.

\subsection{Conclusions}

This chapter has showcased the power of applying patterned nanoparticle for templating biological labels for the cell-response experiment. The advantages brought by patterned nanoparticles are the tunable distance of patterned labels in a flexible and precise range of 30 to $200 \mathrm{~nm}$. The monofunctional nanopattern, i.e., patterning with only AuNPs, has attracted huge research interest and resulted in massive progress in understanding mechanisms in cell responses. However, this type of research has reached its bottleneck of being monofunctionalized. To study more complicated biological affairs or cross-talking of distinct ligand for distancedependent synergism or antagonism, a binary patterned surface is crucially required. Many efforts have been made to develop a binary patterned nanostructure. However, the achieved structure was lacking the spatial correlation between both bio-labels. Being the most critical parameter for these types of experiments, the ability to tune interparticle distance is crucial for any quantitative study. The planet-satellite nanostructure possesses very high potential for this task since its precise control of the distance between planet and satellite nanoparticles. There are few challenges of using silica-planet-gold-satellite nanostructure including finding a suitable substrate and optimizing the condition for functionalization on nanoparticles. Transferring the patterned planetsatellite nanoparticles onto hydrogel makes the binary planet-satellite nanostructure a more general and flexible platform for the biological experiments. The detailed optimization must be conducted together with the biologist in an interactive procedure. 


\section{Part B}

\section{Result and Discussion}




\section{Chapter 4}

\section{Gold-Planet-Silver-Satellite Nanostructures using RAFT Star Polymer $^{1}$}

\subsection{Preface}

The hierarchical self-assembly of distinct nanoelements into precisely ordered nanostructures requires efficient and flexible fabrication strategies. This chapter demonstrate a precise fabrication of bimetallic gold-planetsilver-satellite nanoparticle-arrangements employing RAFT star polymers as particle linker connecting gold nanoparticles (AuNPs) and silver nanoparticles (AgNPs) with judiciously modified surface activity. The strengths of this approach include the adjustability of interparticle distances by tailoring the star polymer molar mass. The prepared nanoassemblies have well-defined structures in which a planet AuNP $(\sim 13 \mathrm{~nm})$ is encompassed by several satellite AgNPs $(\sim 8 \mathrm{~nm})$, thus incorporating the properties of both AuNPs and AgNPs, as confirmed by transmission electron microscopy and UV-Vis spectra. Our results highlight the general applicability of RAFT star polymers as a nano synthesis platform for synthesizing noble metal nanocomposites.

Simultaneous integration of properties from different metal nanoparticles (NPs) by constructing defined three-dimensional nanohybrid structures currently attracts great interest in materials science.[108-115]

\footnotetext{
${ }^{1}$ Adapted with permission from Peng, W; Vana, P. ACS Macro Letters 2016, 5, 1227-1231. Copyright 2016, American Chemical Society, Washington, DC, USA. Text is adjusted to match the formatting of this thesis
} 
Among the various metal nanocomposites, gold nanoparticles (AuNPs) and silver nanoparticles (AgNPs) are of huge interest due to their unique electronic, optical, and catalytic characteristics. ${ }^{[3,116]}$ Assembling both types of NPs into the planet-satellite nanohybrids may induce collective properties that differ from those of individual particles such as plasmonic coupling[29] and amplified SER[117] (surface enhanced raman) properties. The tunneling charge-transfer between AuNPs and AgNPs induced by the local potential gradient between both nanocomposites was also reported.[118] To achieve the formation of planet-satellite nanostructures incorporating AuNPs and AgNPs, various synthetic strategies can be applied: Electrostatic interactions, ${ }^{[20-22]}$ metal-ion-mediated complexation ${ }^{[23,24]}$ and application of "click chemistry"[25] could result in straightforward attachment of satellite particles onto the surface of the nano-planet. Sulfurcontaining molecular linkers ${ }^{[29,119]}$ especially DNA components ${ }^{[120,121]}$ are also widely applied for the fabrication of planet-satellite nanostructures from AgNPs and AuNPs with small interparticle distance $<10 \mathrm{~nm}$. Large ( 100 nm range) interparticle distance was accessible by applying DNA origami scaffolds ${ }^{[35]}$ acting as giant molecular linker. But the production is limited by virtue of the complicated experimental procedure, high cost of the DNA compounds, and the small scale at which the materials can be obtained. Herein, the synthetic functional polymers are employed for the task of programming the different metal NPs into one precise hierarchical nanostructure, as we know about the capabilities of tailored synthetic polymers as spacer and stabilizer for nanoparticles from our earlier work. ${ }^{[17]}$ Applying polymer as macromolecular linker provides several advantages: Diverse functional polymers such as stimuli-responsive polymers[53,122-126] can be integrated into the nanostructures and equip them with a specific function. To obtain a suited polymeric NP linker, a special chemical and topological design of the polymer structure is required. Here, the general aurophilicity and argentophilicity of sulfur-containing end-groups (i.e., in this case, trithiocarbonates) from RAFT (reversible addition-fragmentation 
chain transfer) polymerization[127] is exploited, which enables efficient adsorption of polymers on surfaces of AgNPs ${ }^{[128,129]}$ and AuNPs ${ }^{[17]}$. Beyond the inherent end-group functionality of the obtained polymer, the RAFT technique also enables a controlled polymerization process, resulting in polymers with pre-determined average molar masses and narrow molar mass distributions. Therefore, the possibility of obtaining polymers with distinct molar masses enables fine control of the interparticle distance in the planet-satellite nanostructures [41,42]. Another and most important challenge to design a polymeric NP linker is the topological structure of the polymers: Polymeric linkers with multiple NP binding sites such as hyperbranched[40] and star-shaped RAFT[41] polymers were reported to be suitable for this task. Our group has reported a method to prepare gold-planet-gold-satellite nanostructures using 4-arm star polymers decorated with trithiocarbonate groups on their chain termini. [41,42]

\subsection{Self-assembly of gold-planet-silver-satellite nanostructures}

In this section, a gold-planet-silver-satellite nanohybrid structure (Figure 4.1) is constructed based on the unique properties of star RAFT polymers (Figure 4.1b). Star poly- $N$-isopropylacrylamide (PNiPAAM) is chosen here due to its general good solubility in water and organic solvents and its good applicability in RAFT polymerizations. Two types of polymer with different topology were synthesized to serve different purposes: As the polymeric linker between AuNPs and AgNPs, RAFT-PNiPAAM with star topology was synthesized practically identical to a previously reported method. The chain length of the polymer arms was varied in order to tune the interparticle distance (Au-Ag) in the planet-satellite nanohybrids.[41] To protect the nanohybrids from aggregation, RAFT-PNiPAAM with linear topology was obtained from monofunctional RAFT agent 1 (Figure 4.1a).[130] The molar mass distributions of the RAFT polymers (Figure $4.1 \mathrm{~b}$ ) were 
obtained by size-exclusion chromatography (SEC). The hydrodynamic volume of the star polymer is smaller than that of its linear counterpart with equal molar mass, as a consequence of the star geometry. In order to correct the average molar mass of star polymers from SEC analysis, which is calibrated using linear polymer standards, a factor of 1.3 is multiplied to the average molar mass obtained from SEC for the 4 -arm star polymers.[131] The results from SEC analysis are listed in Table 4.1.

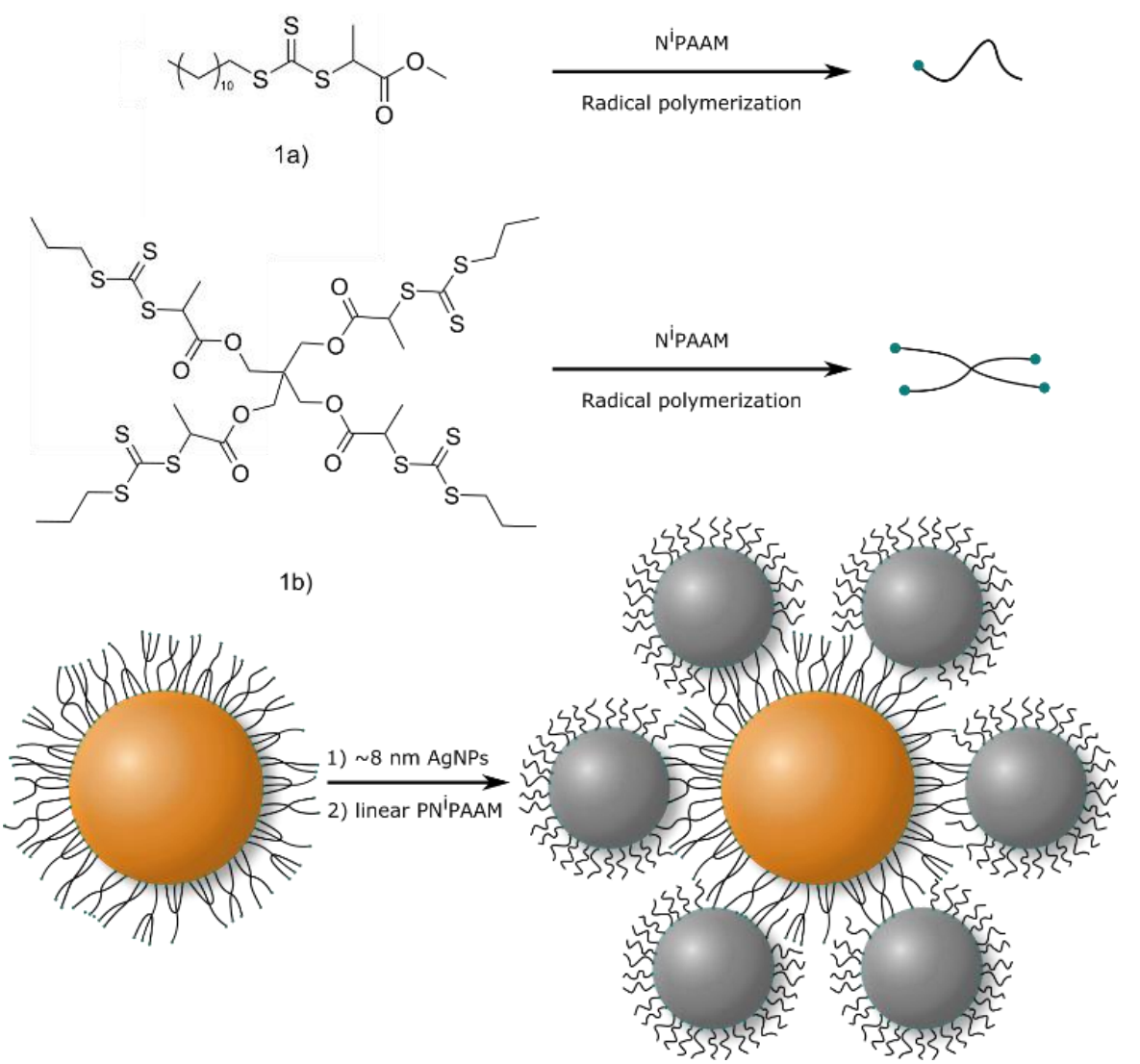

Figure 4.1: Synthetic scheme for the preparation of gold-planet-silver-satellite nanoassemblies from star poly(NiPAAM) functionalized hybrid gold nanoparticles. The RAFT groups are illustrated by green cycles. Linear (1a) and star-shaped (1b) RAFT agents are used in this work for the preparation of PNiPAAM with different topology. Adapted with permission from ref. [74]. Copyright 2016 American Chemical Society. 
( $13 \mathrm{~nm}$ ) AuNPs from citrate-reduction was chosen as the "planet". The RAFT polymers were immobilized on the surface of AuNPs via a grafting-to approach.[17,41,42] After the removal of the excess polymer via three centrifugation/redispersion cycles, the star-PNiPAAM-capped AuNPs were dispersed in $\mathrm{CHCl}_{3}$. The polymer-functionalized AuNPs were then analyzed by TEM (Figure 4.6) and DLS (Figure 4.7). In TEM, an increase of the interparticle distance $(\mathrm{Au}-\mathrm{Au})$ with an increasing chain length of the star polymer is observed as already reported elsewhere. ${ }^{[41,42]}$ DLS results also show a remarkably increased particle diameter induced by the grafting with polymers compared with the citrate-capped AuNPs (Figure 4.7C). The star PNiPAAM immobilized on the surface of AuNPs could still have free arms which are not bound onto the surface. These free arms carrying trithiocarbonate groups at their chain ends have the ability to attach to other NPs, for example, $\sim 4 \mathrm{~nm}$ Brust-Schiffrin AuNPs. ${ }^{[41,42]}$

Now, however, we target Ag: To choose a suitable type of AgNPs as satellites, surface oxidation and the electrical charge have to be considered. In our first experiments conducted in aqueous phase with citrate-capped AgNPs, planet-satellite structures were not yielded. This may be caused by the repulsive force between negatively charged AgNPs which impedes AgNPs from approaching each other in the nano-range. Consequently, oleylamine (OA)-capped AgNPs ( $\sim 9 \mathrm{~nm}$ ) were chosen as satellites for their good solubility in organic solvents and their appropriate surface activity. It is reported that the OA ligand is weakly bound on the surface of AgNPs and can be replaced by thiols. ${ }^{[132]}$ In order to avoid the oxidation of the silver surface, the synthesis is conducted under argon atmosphere, as it is experimentally observed that AgNPs synthesized under ambient air show different behavior and lower surface activity than those synthesized under inert atmosphere (details in experimental chapter). Our experiments show that the capping density of $\mathrm{OA}$ on AgNPs has a remarkable effect on the activity of AgNPs, which further affects the character of the planet-satellite structure. Two different types of OA-capped AgNPs are applied to gain 
insight into this phenomenon: One type of AgNPs is labeled "AgNPs washed $2 x^{\prime \prime}$ which is obtained from 2 centrifugation/redispersion cycles after the synthesis; the other is labeled "AgNPs-washed-3x", which is derived from "AgNPs-washed-2x" after one additional centrifugation cycle. These two types of AgNPs have largely different stability in colloidal dispersions and different $\mathrm{OA}$ surface density. For example, in $\mathrm{CHCl}_{3}$, the three-times washed AgNPs tend to aggregate upon dilution and cannot be redispersed in hexane; this is in contrast to the behavior of "AgNPs-washed-2x". The washing of particles, which governs the particle surface activity, is thus a critical procedure. Efforts were made to obtain "AgNPs-washed-4x", but all attempts have reproducibly resulted in black aggregates after the 4th centrifugation cycle due to insufficient surface protection. On the other hand, applying "AgNPs-washed-1x" or AgNPs directly from the synthesis will not result in effective binding of AgNPs onto star-polymer-capped AuNPs, due to the small surface activity of these AgNPs. Details of purifying the AgNPs can be found in the experimental chapter.

The fabrication of gold-planet-silver-satellite nanohybrids is experimentally very straight forward: By adding large excess (approximately 30x) of OA-capped AgNPs dispersed in $\mathrm{CHCl}_{3}$ to the colloidal dispersion of star-PNiPAAM-capped AuNPs in $\mathrm{CHCl}_{3}$, the formation of goldplanet-silver-satellite structures will proceed without further treatment. After 30 min of incubation, excess of linear PNiPAAM RAFT polymer was added to protect the structure from aggregation (see Figure 4.1). The heavier planet-satellite structures were separated from the excess of lighter AgNPs and linear PNiPAAM by three centrifugation/redispersion cycles $\left(2150 \mathrm{~g}, 1 \mathrm{~h}, 5^{\circ} \mathrm{C}\right)$. The addition of protecting linear PNiPAAM and lower centrifugation speed was found to be crucial in the synthesis. Experiments conducted without linear polymer or under high centrifugation speed $(15300 \mathrm{G})$ resulted in strong cross-linking between planet-satellite structures. The as-synthesized gold-planet-silver-satellite nanostructures were analyzed by TEM (Figure 4.2 and Figure 4.3). TEM images show that 
these planet-satellite nanostructures tend to self-assemble on the TEM substrate and form well-defined 2D structures, in which Au and Ag tend to precisely alternate. High-angle annular dark-field scanning transmission electron microscopy (HAADF-STEM) confirmed the alternating gold-silver composition of the nanohybrids (Figure 4.4).

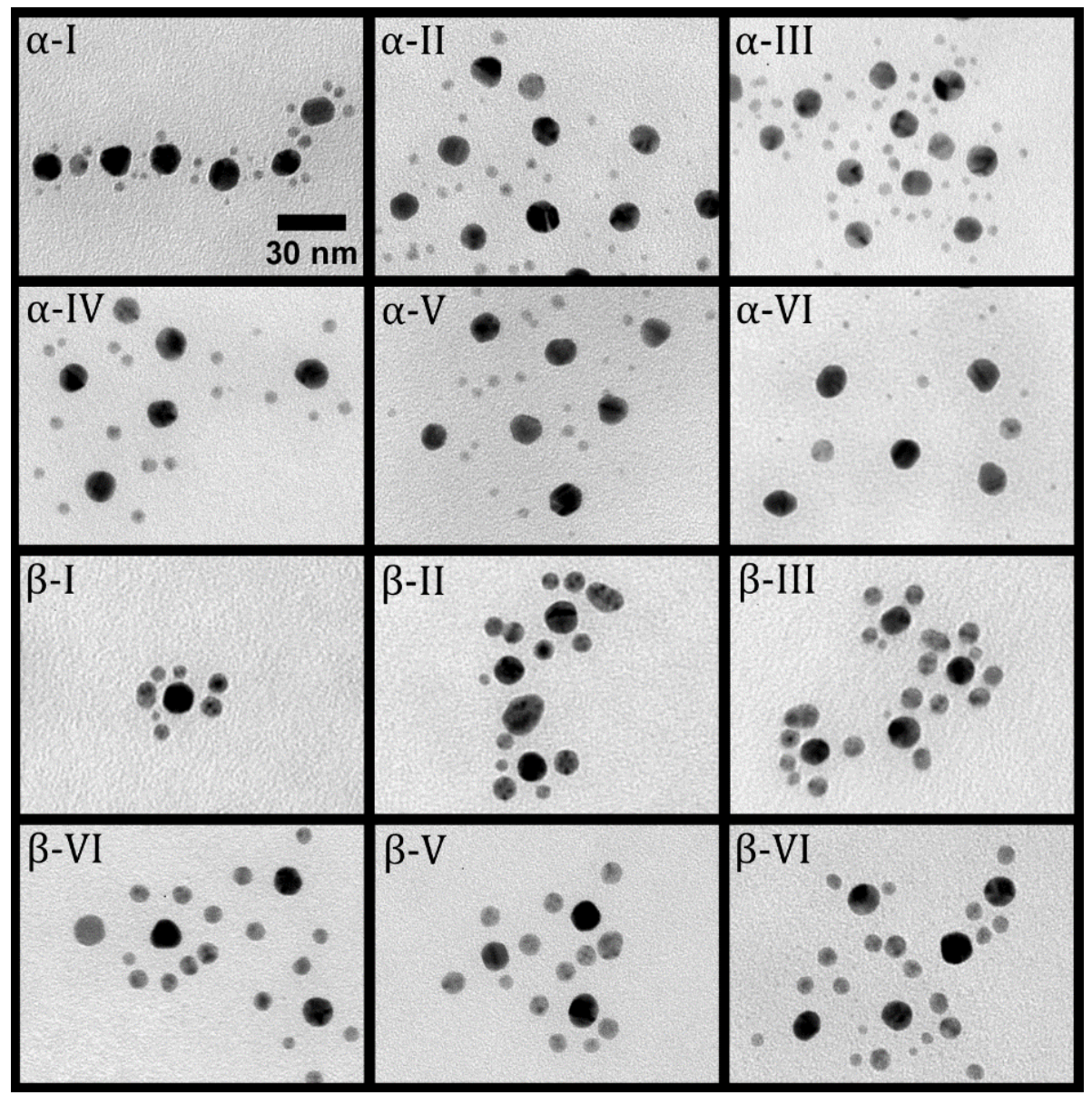

Figure 4.2: TE micrographs of gold-planet-silver-satellite nanostructures. Samples labeled as $\alpha$ resulted from "AgNP washed 3x", samples labeled as $\beta$ are resulted from "AgNP washed 2x". Adapted with permission from ref. [74]. Copyright 2016 American Chemical Society.

The average interparticle distance ( $\mathrm{Au}-\mathrm{Ag}$ ) was extracted from TEM images. Figure 4.5 shows the dependence of the interparticle distance (Au$\mathrm{Ag}$ ) on the number average molar mass of the star polymer linker. 
Interestingly, AgNPs with different capping density of OA result in remarkably different interparticle distances (Figure 4.2 and Figure 4.3): "AgNPs-washed-2x" with 19\% OA of its total weight (TGA, see Figure 4.8) result in shorter interparticle distances (samples labeled $\beta$-I to VI, derived from star-PNiPAAM I-VI, respectively) than that from "AgNPs-washed-3x" with lower (10\%) OA content (samples labeled $\alpha$-I to VI). This indicates that the coverage of OA on AgNPs has a pronounced influence on the distance scaling behavior of the resulting planet-satellite structures. The "activity" of the AgNPs is clearly enhanced when the capping agents are partly removed. It can be speculated that the higher activity of the AgNPs then results in a more effective binding on the exterior of the star polymer shell on AuNPs, i.e., more linking star PNiPAAM is present between the Au-planet and the Agsatellite. As a consequence of that, the AgNPs with lower surface activity appears to penetrate deeper into the star polymer shell on central AuNPs (Figure 4.5), at least in the dried state after casting on the amorphous carbon surface used for TEM characterization. It is fully consistent with this line of thoughts that the interparticle distances of the gold-planet-gold-satellite nanostructures, which was reported recently, ${ }^{[41,42]}$ are larger than with the here reported AgNPs (see Figure 4.5), as the Brust-Schiffrin AuNPs as satellite particles (with weakly bound tetraoctylammonium bromide ligands ${ }^{[133]}$ instead of amine ligands) are more "active".

Table 4.1: Results from SEC analysis of the different polymer samples. Samples Star-II and Star-IV were previously used in our earlier publication.[41,42]

\begin{tabular}{llllll}
\hline Polymer & Star-I & Star-III & Star-V & Star-VI & Linear \\
\hline$M_{\mathrm{N}} \mathrm{g} / \mathrm{mol}$ & $29 \mathrm{k}$ & $44 \mathrm{k}$ & $60 \mathrm{k}$ & $78 \mathrm{k}$ & $30 \mathrm{k}$ \\
\hline$\emptyset_{\mathrm{M}}$ & 1.1 & 1.1 & 1.1 & 1.1 & 1.1 \\
\hline
\end{tabular}




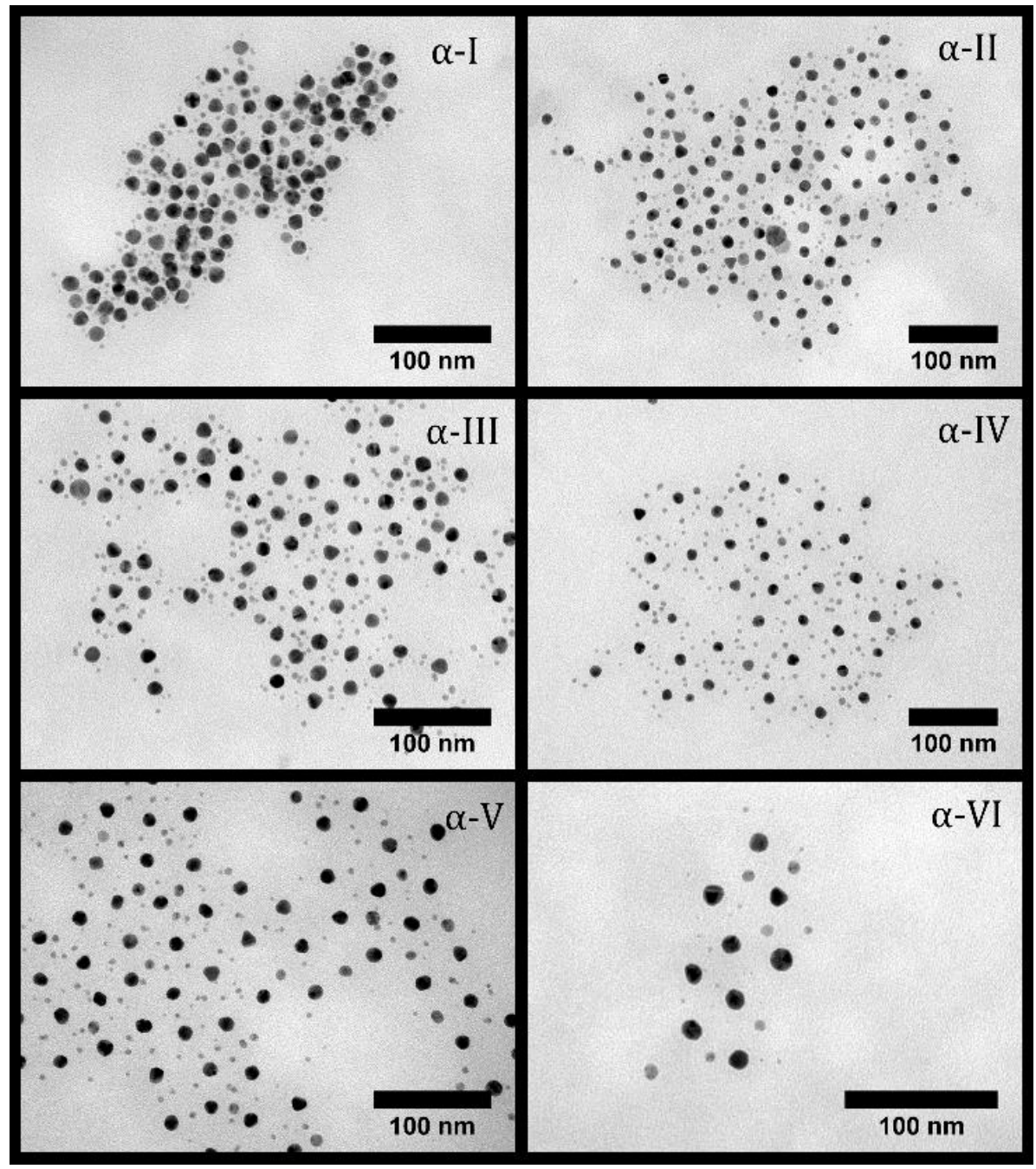

Figure 4.3: TE micrographs of gold-planet-silver-satellite nanostructures $\alpha$-I to VI in lower magnification. Adapted with permission from ref. [74]. Copyright 2016 American Chemical Society. 

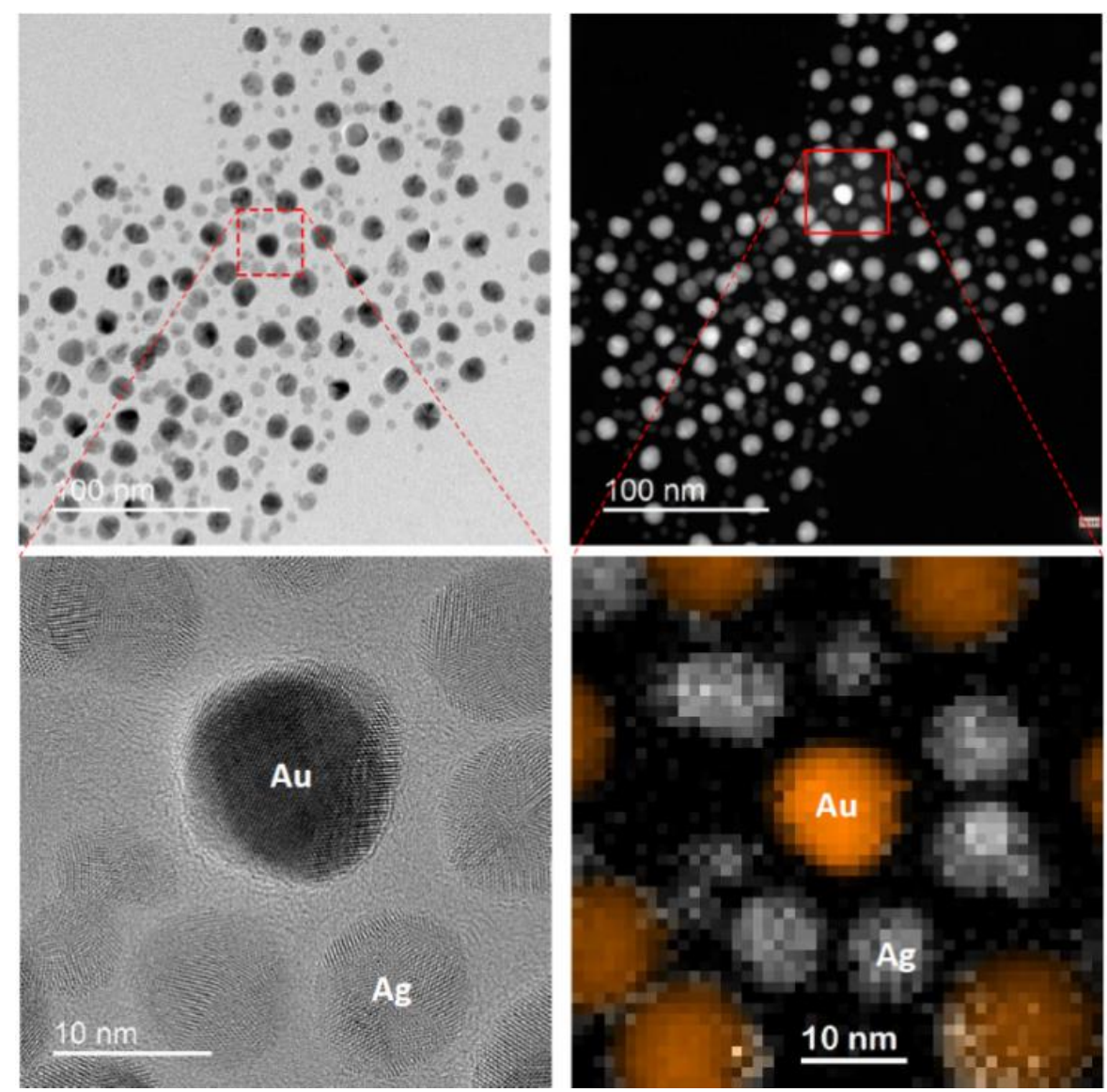

Figure 4.4: Overview bright-field (upper left) and dark-field (upper-right) image. The images on the bottom show magnified details in a high-resolution bright-field image (left) and in an elemental map obtained via HAADF-STEM (right) for a specimen of gold-planet-silver-satellite nanostructures. Adapted with permission from ref. [74]. Copyright 2016 American Chemical Society.

In addition to HAADF-STEM, UV-Vis spectra of the nanohybrids show the existence of characteristic plasmon bands both for AuNPs $(\sim 520 \mathrm{~nm})$ and AgNPs ( 410 nm) (Figure 4.9). Comparing the UV-Vis spectra of the planet-satellite nanohybrids with the linear combinations of spectra of individual AuNPs and AgNPs, red-shift and increased absorbance caused by the coupling of the surface plasmon resonance between the NPs that are in close spatial proximity is observed in all cases. In general, remarkable plasmonic coupling first occurs at a particle distance comparable to the 
diameter of the particles. ${ }^{[134,135]}$ The change in the UV-Vis spectra, therefore, validates the nanoscaled interparticle distance (Au-Ag) on the planetsatellite nanohybrids also in solution. It can also be seen by TEM and in UVVis spectra that using star-PNiPAAM with lower molar masses (for example, in samples $\beta$-I, $\beta$-II, see Figure 4.9) causes aggregation of the planet-satellite structures, probably due to insufficient colloidal stability in these systems. In contrast, no observable aggregated material was present in the samples synthesized from long star polymer linkers (star-III, IV, V and VI). Here, the produced nanostructures were stable for at least 4 months when stored sealed in tinted glass and dispersed in chloroform at room temperature. Thus, there seems to be a critical arms' length for the particle linker to provide sufficient colloidal stability to the overall nanostructure and to prevent the planet-satellite structure from aggregating under the experimental conditions applied here. Besides, the planet-satellite nanostructures derived from AgNPs washed 2x ( $\beta$ samples) had a stronger tendency to aggregate when short polymeric linker (Star-I, II) was used. This experimentally observed tendency again indicates a relatively poor capping with protective linear PNPAAM on the outer surfaces due to the lower surface activity of AgNPs in the $\beta$ group. 


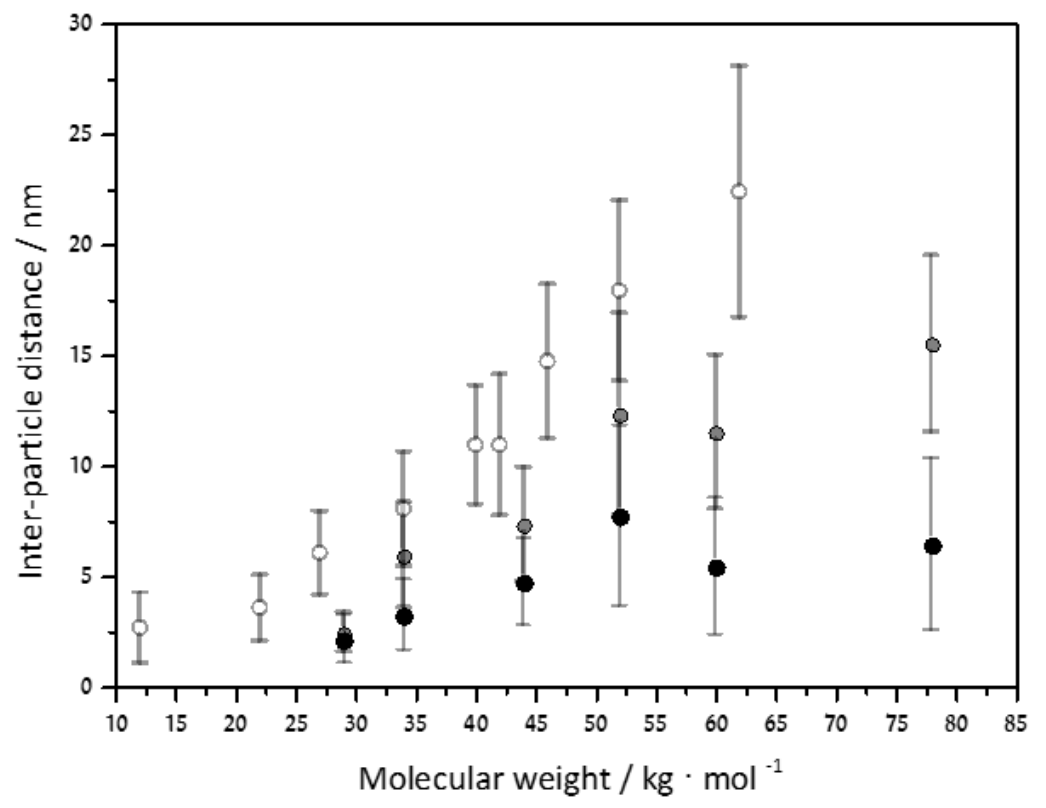

Figure 4.5: Edge-to-edge distances of gold-silver nanohybrid particles $\alpha$-I to VI (gray circles) and $\beta$-I to VI (black circles) vs. the number average molar mass of the linking star polymer. For comparison, the interparticle distances from gold-planet-goldsatellite nanostructures from Rossner and Vana using a similar synthetic approach are also plotted as empty cycles. Adapted with permission from ref. [74]. Copyright 2016 American Chemical Society. 


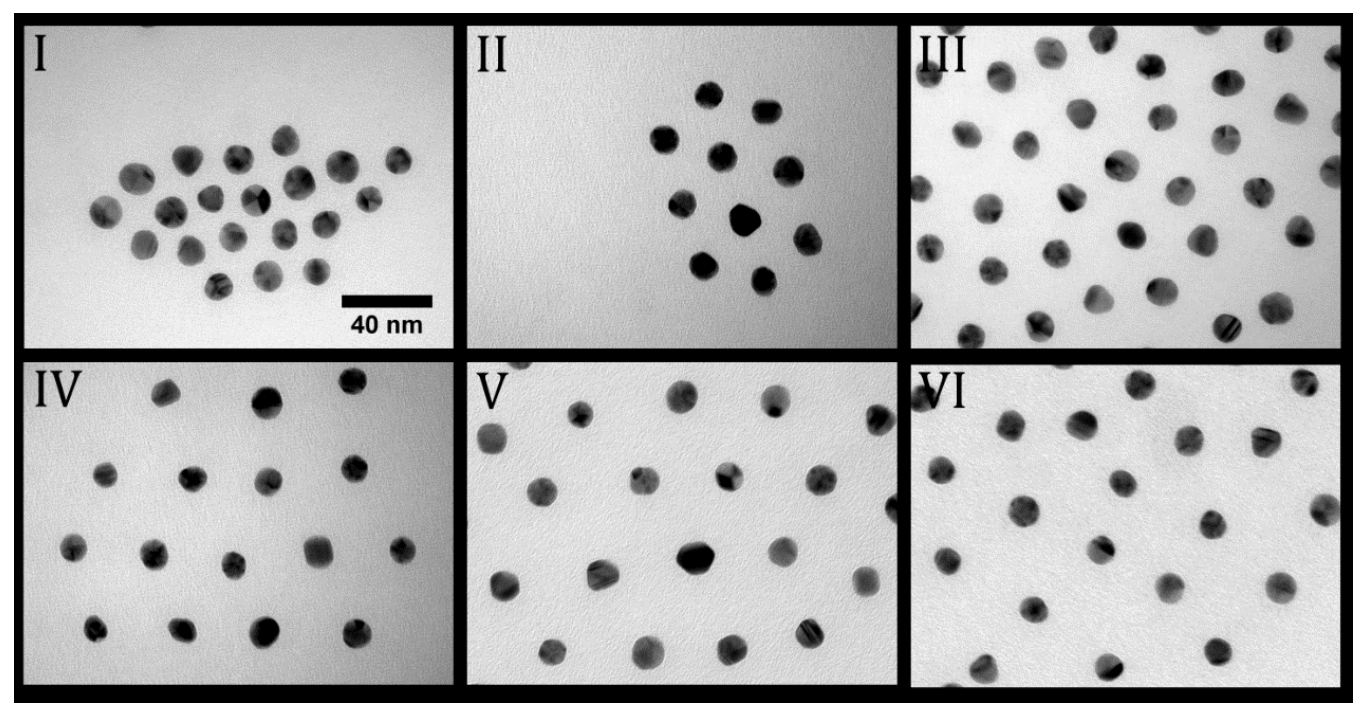

Figure 4.6: TE micrograph of star-PNiPAAM-I-VI-capped AuNPs. An increase of interparticle distance can be observed. Adapted with permission from ref. [74]. Copyright 2016 American Chemical Society. 

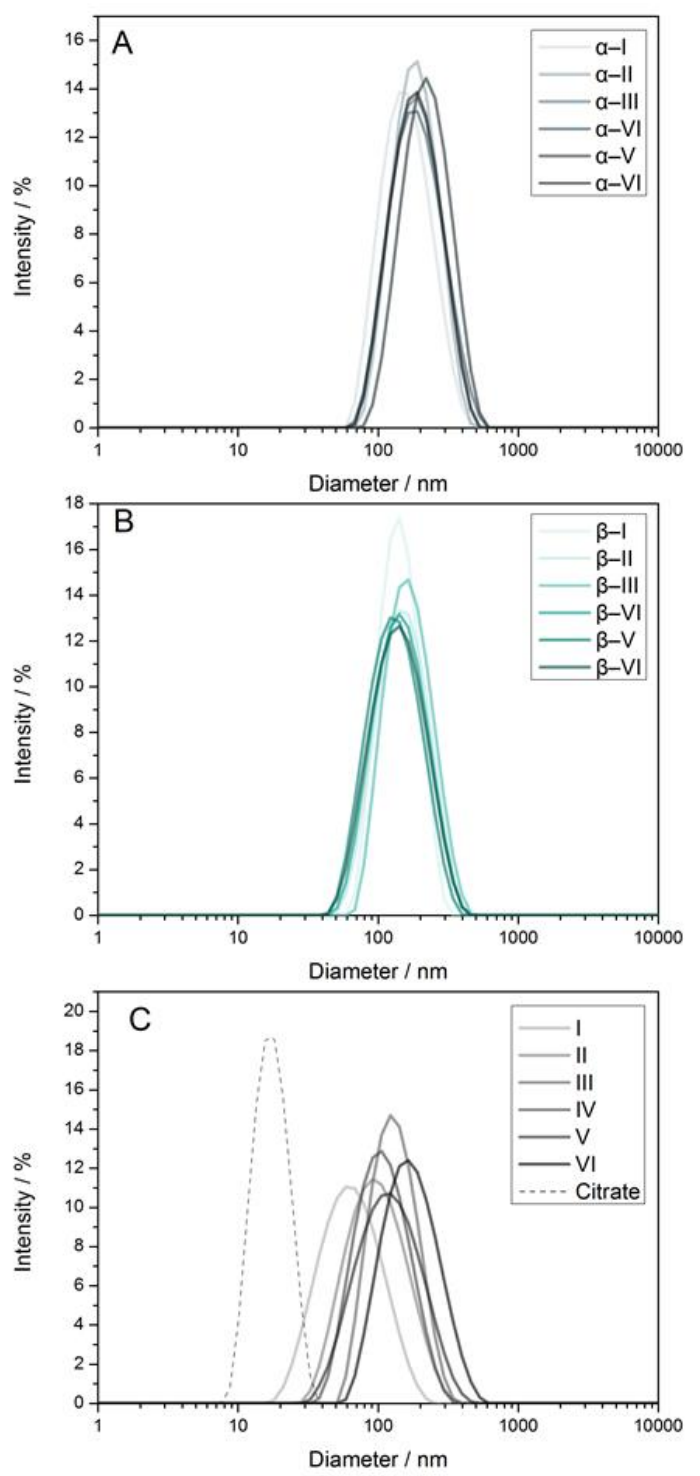

Figure 4.7: Intensity distribution from DLS for the gold-planet-silver-satellite nanostructures I-VI yielded from AgNP-washed 3x (Figure labeled A) and AgNPwashed $2 \mathrm{x}$ (Figure labeled $\mathrm{B}$ ). Figure labeled $\mathrm{C}$ is the intensity distribution of the starPNiPAAM-I-VI-capped AuNP, and the dashed line is the result of citrate-capped AuNPs. All the samples were passed through a $0.45 \mu \mathrm{m}$ PTFE filter prior to the DLS experiment. Adapted with permission from ref. [74]. Copyright 2016 American Chemical Society. 


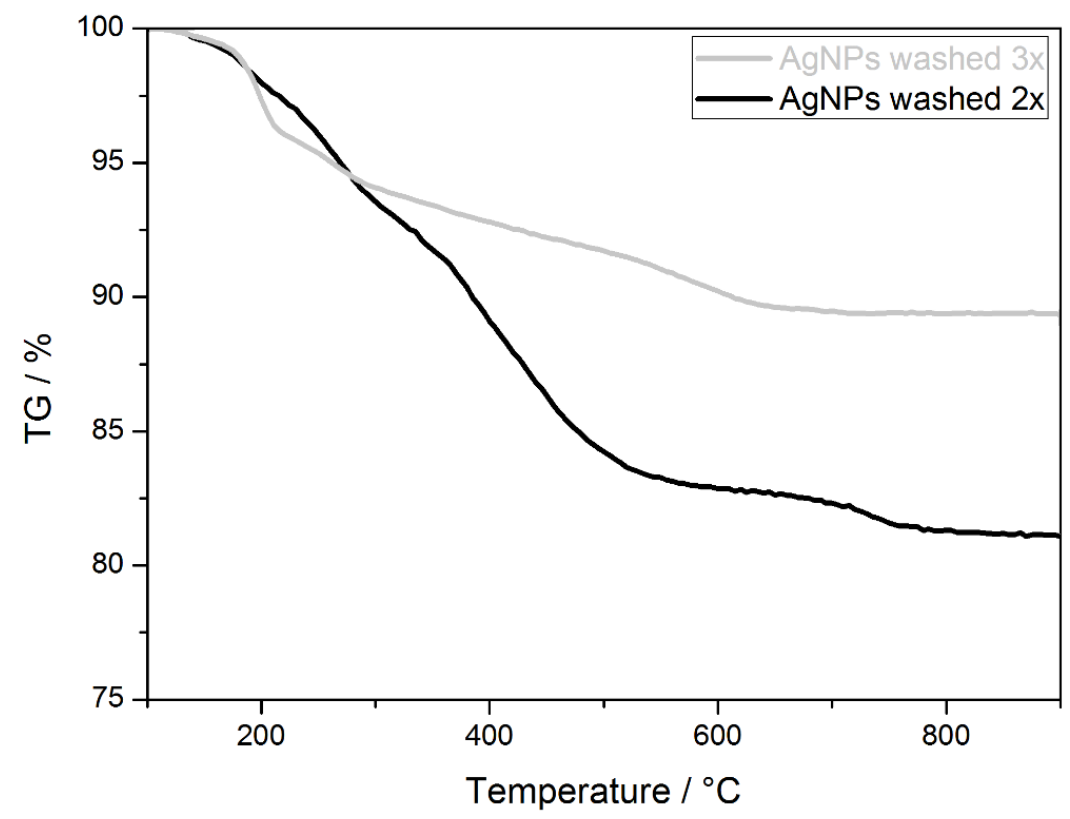

Figure 4.8: TGA curve of OA-capped "AgNPs washed 2x" / "AgNPs washed 3x". Adapted with permission from ref. [74]. Copyright 2016 American Chemical Society. 

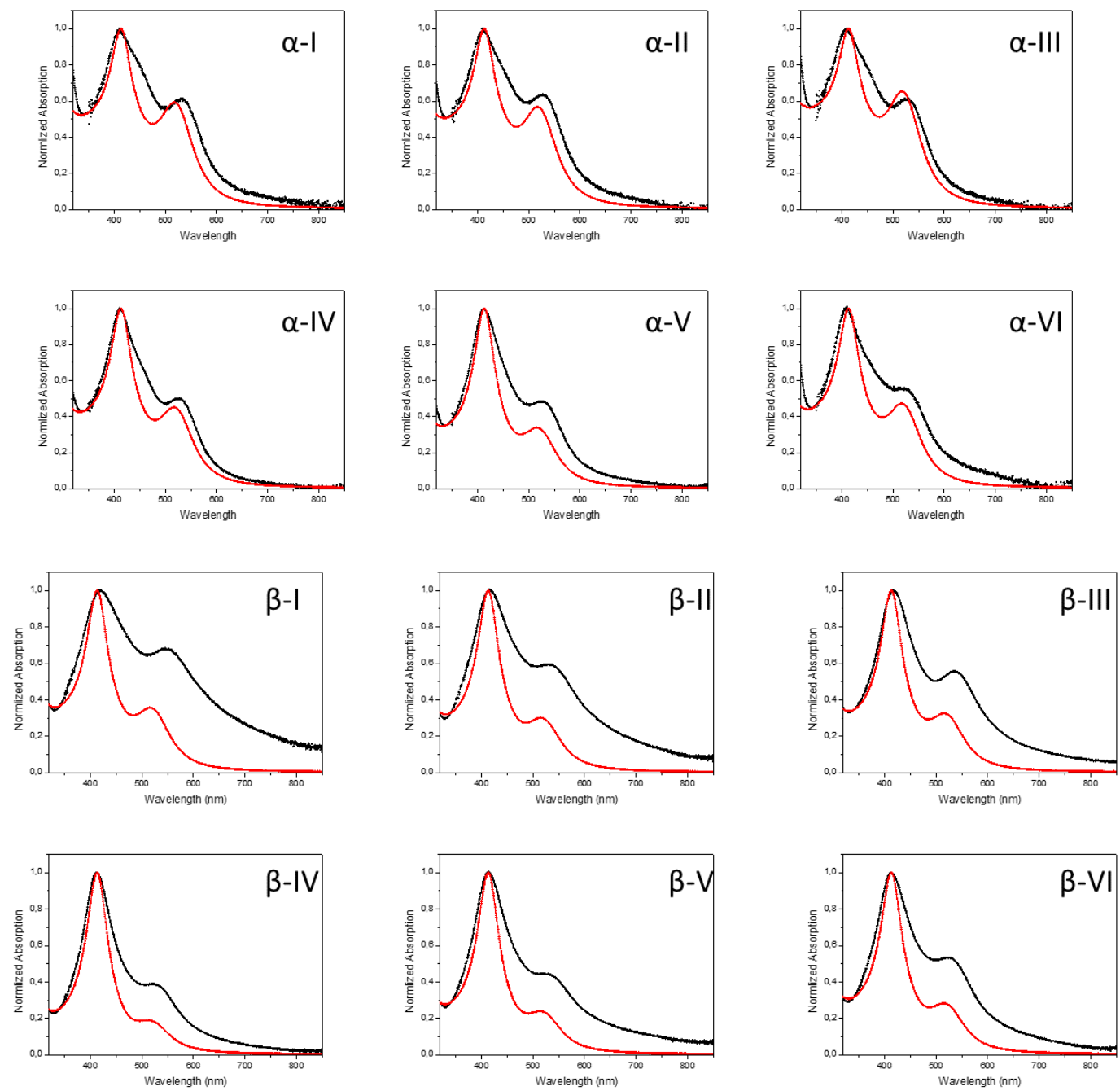

Figure 4.9: UV-Vis spectra of samples $\alpha-I-V I$ and $\beta$-I-VI. The black curve is measured experimentally without any filtration. The red curve is obtained by a linear combination of spectra from individual $\sim 13 \mathrm{~nm}$ AuNPs and $\sim 9 \mathrm{~nm}$ AgNPs. The boundary conditions for the linear combination is matching the absorbance at the wavelengths of $340 \mathrm{~nm}$ and $412 \mathrm{~nm}$. The strong additional absorption on the spectra of the sample $\beta$-I and $\beta$-II comes from the aggregated network structure which is observed by TEM (Figure 4.10). Adapted with permission from ref. [74]. Copyright 2016 American Chemical Society. 


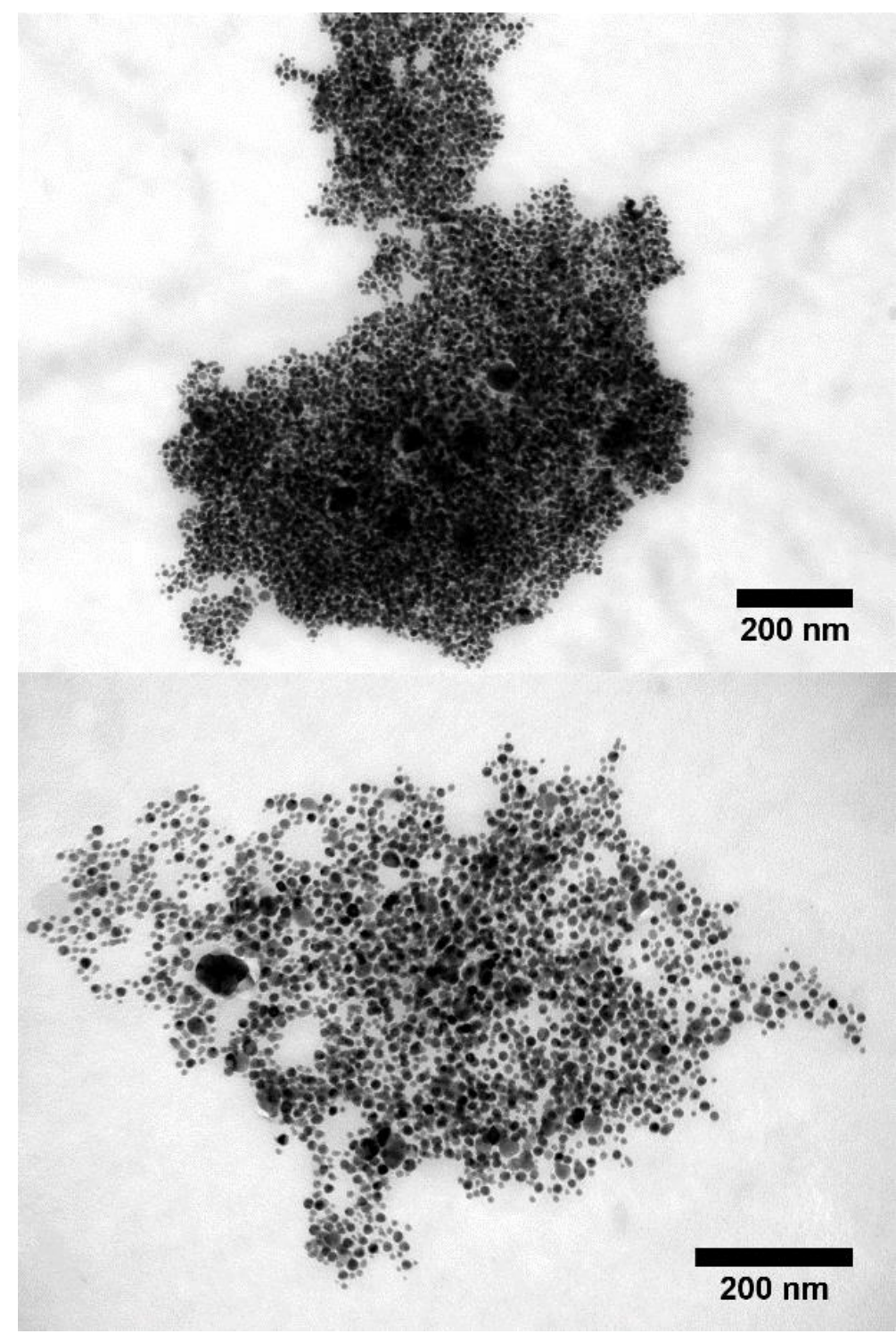

Figure 4.10: TE micrograph of aggregated nanostructures from samples $\beta$-I (left) and $\beta$-II (right). Adapted with permission from ref. [74]. Copyright 2016 American Chemical Society. 

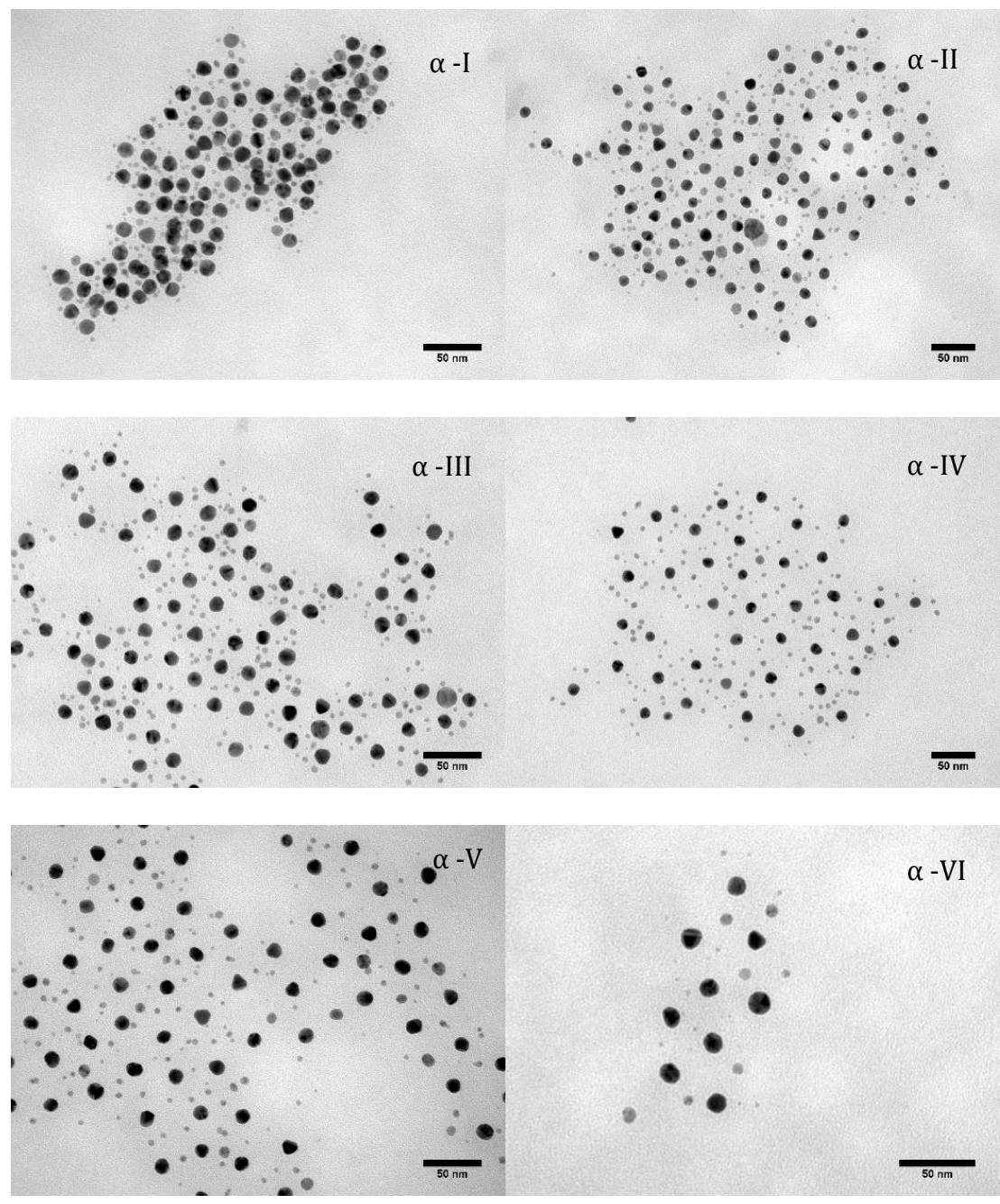

Figure 4.11: TEM images of gold-planet-silver-satellite nanohybrid in lower magnification for $\alpha$-I to $\alpha$-VI. 

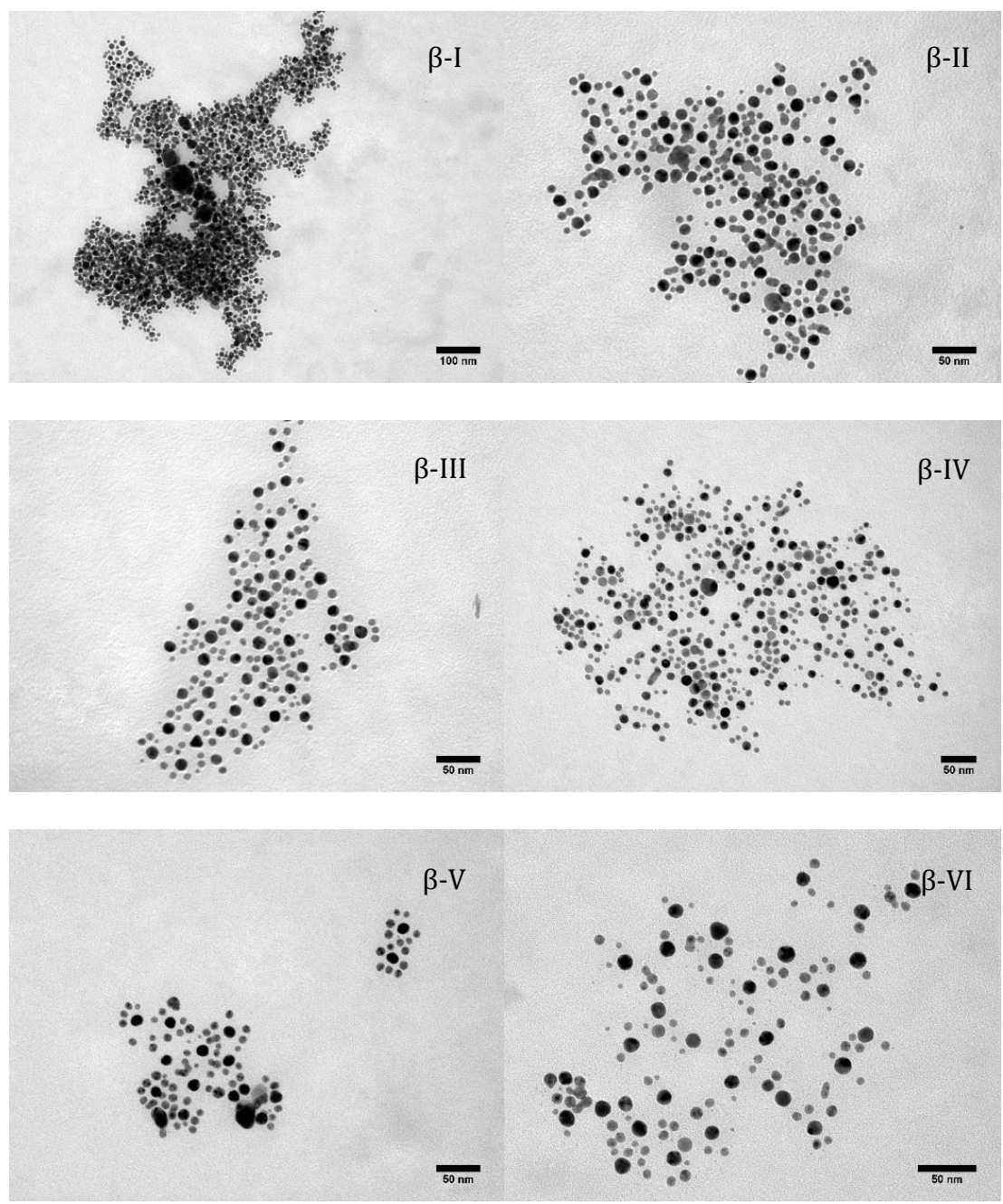

Figure 4.12: TEM images of gold-planet-silver-satellite nanohybrid in lower magnification for $\beta$-I to $\beta$-VI. Adapted with permission from ref. [74]. Copyright 2016 American Chemical Society.

\subsection{Conclusion}

In conclusion, a straightforward and effective synthesis is established for the fabrication of gold-planet-silver-satellite double plasmonic nanoassemblies with tunable interparticle distances mediated by star RAFT PNiPAAM. RAFT star polymers possess the general ability to connect 
different noble metal NPs and enable the control of the arrangement structure, e.g., a precisely alternating arrangement of $\mathrm{Au}$ and $\mathrm{Ag}$ nanoparticles in the present study. This demonstrates that RAFT star polymers are powerful and extremely versatile modular building units that can be used as particle linker and defined spacer for the formation of tailored nanoarchitectures. Additionally, the adjustable activity of OAcapped AgNPs by variation of the capping density of OA offers another handle to tune NP reactivity and resulting assembly structures. 


\section{Chapter 5}

\section{Fabricating Silica-Planet-Gold-Satellite Nanostructure for Binary Nano-Patterning}

\subsection{Preface}

Fabricating a binary nanopatterned surface as a template for orthogonal binary chemical/biological labeling requires nanocomponents to feature completely distinct chemical characters. In this thesis, silica nanoparticle with a diameter of $\sim 40 \mathrm{~nm}$ was chosen as nano-planet for the highly efficient silane chemistry on its surface. Gold is chosen as a nanosatellite for its convenient thiolphil character. Further reasons to choose silica and gold as the candidates is their ability to survive plasma treatment (to remove polymer) while maintaining their surface chemical activity for further functionalization.

This chapter focuses on the fabrication of a well-defined silica-planetgold-satellite nanostructure. The procedure starts with the synthesis of highly spherical silica nanoparticles from w/o reverse microemulsion technique and following up anchoring of RAFT agent (section 5.2.1). The RAFT capped $\mathrm{SiO}_{2}$-NPs are able to conduct surface-induced RAFT polymerization to grow polymer from the surface. The condition of polymerization with NiPAAM must be optimized in detail to archive the synthesis of fully separated polymer capped silica planet with tunable distance (section 5.2.2). The self-assembly of the silica-planet-gold-satellite nanostructure can be done by simply mixing polymer capped silica with AuNPs in a suitable solvent. The as-synthesized planet-satellite nanostructure can self-assembly on the substrate forming highly ordered hexagonal pattern. By changing the length of the linker polymer, the 
interparticle can be fully controlled (section 5.3). After the synthesis of the planet-satellite nanostructure, the solvent and concentration are changed to meet the condition for dip-coating on the substrate. The homogenous monolayer formation of the planet-satellite nanopattern on the $\mathrm{CaF}_{2}$ surface is successful as indicated by AFM and SEM. The polymer content is then removed by plasma treatment for further functionalization with cyclic RGD peptide (section 5.4). PEGylation on nanopatterned $\mathrm{CaF}_{2}$ surface are attempted for the biologic passivation for melanoma cells and cyclic RGD peptide. The passivation is sufficient for melanoma cells, however, not sufficient for peptide labels (i.e., cyclic RGD). To address this issue, a novel strategy to transfer the binary nanopattern onto PEG hydrogel is established. By choosing suitable linkers for both AuNPs and silica NPs and optimizing conditions for functionalization, both types of nanoparticles are able to immobilize onto the surface of hydrogel.

\subsection{Nano-planet: surface-induced RAFT polymerization from silica nanoparticles}

This section focuses on the surface-induced RAFT polymerization from the surface of silica nanoparticles ( $\sim 38 \mathrm{~nm})$. RAFT group is first introduced onto the surface of $\mathrm{SiO}_{2}$-NPs via a two-step reaction by first anchoring the $\mathrm{NH}_{2}$ group and successive RAFT agent via $-\mathrm{NH}_{2} /$ mercaptothiazoline click chemistry. The RAFT decorated $\mathrm{SiO}_{2}$-NPs is then ready for surface-inithiated polymerization by dispersing the particles in the polymerization solution. Although the chemical approach has already been reported, the specific condition including choice of solvent, handling of nanoparticles and pretreatment of the polymerization's colloid must be studied in every detail to achieve a perfectly defined high-density polymer capped silica NP as "nanoplanet." 


\subsubsection{Introducing RAFT group onto the surface of $\mathrm{SiO}_{2}$ surface}

There is a great variety of methods to synthesize $\mathrm{SiO}_{2}$ nanoparticles. The ideal particles for the task as nano-planet should be highly spherical, completely separated and have a diameter below $50 \mathrm{~nm}$. $\mathrm{SiO}_{2}$-NPs synthesized from reversed microemulsion (hexane/water) technique using IGEPAL CO-520 as surfactant meet all the requirements mentioned above. The narrow size distribution (typically $38 \pm 1 \mathrm{~nm}$ ) gives a highly uniform geometry as nano-planet (Figure 5.1).

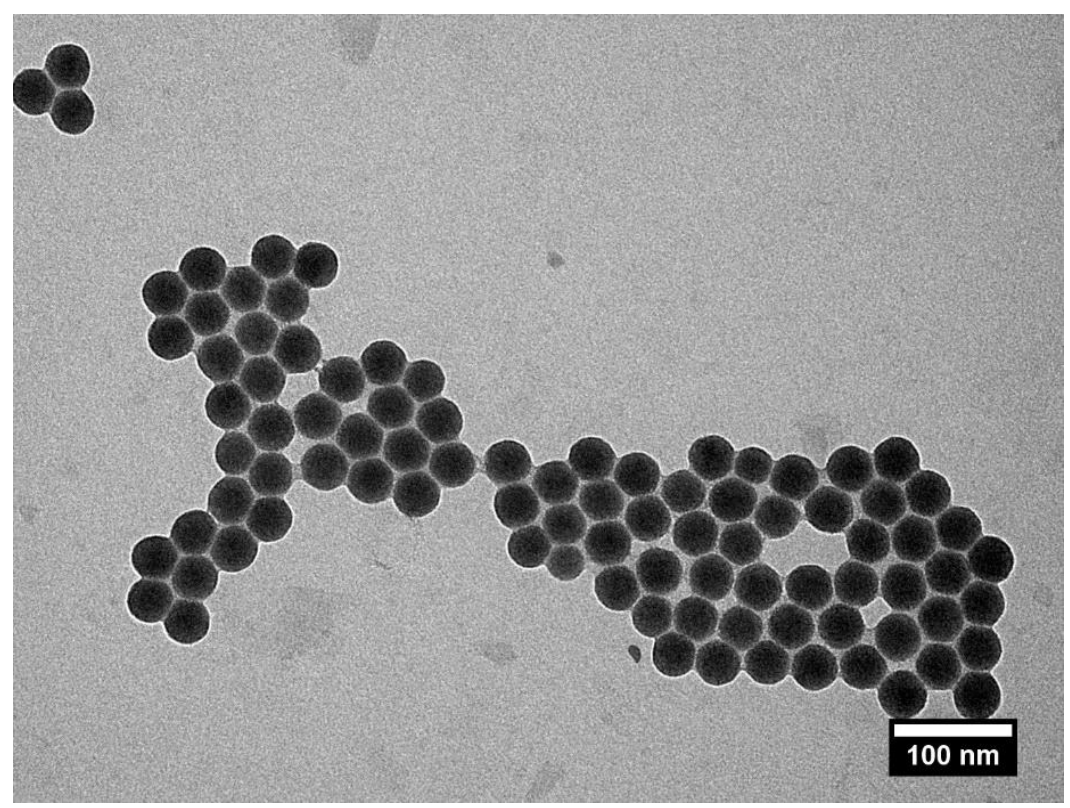

Figure 5.1: TE Micrograph of $\mathrm{SiO}_{2}$-NPs synthesized from the reversed emulsion technique. The particles possess high roundness and uniform size distribution ( $39 \pm 1 \mathrm{~nm}$, size distribution in section 7.3). All $\mathrm{SiO}_{2}$-NPs are completely separated from each other.

It is well-known that silica possesses a significant number of $\mathrm{OH}$ groups on its surface ${ }^{[136]}$ which can be used for silane chemistry to introduce the functional group. Base on this strategy, the RAFT group can be efficiently bunded to the surface of the $\mathrm{SiO}_{2}$-NPs with very high density. 
Here, a two-step reaction (Figure 5.2) is chosen by first anchoring the $\mathrm{NH}_{2}$ group onto the $\mathrm{SiO}_{2}$-NPs surface by mixing nanoparticles with 3aminopropyldimethylethoxysilane in refluxing anhydrous THF under argon atmosphere. The excess of chemicals can be easily removed by the centrifuge/redispersion process. After purification, a trithiocarbonate based RAFT agent featuring a mercaptothiazoline end group (activated RAFT) is mixed with the $\mathrm{NH}_{2}$ functionalized $\mathrm{SiO}_{2}$-NPs in dry THF to obtain RAFT functionalized $\mathrm{SiO}_{2}$-NPs. The detailed experimental procedure can be found in section 7.4.

The RAFT functionalized $\mathrm{SiO}_{2}$-NPs possess enhanced dispersibility in organic solvent providing a more rational polymerization by perfectly dispersing the RAFT capped $\mathrm{SiO}_{2}$-NPs in polymerization's solution. The assynthesized RAFT functionalized $\mathrm{SiO}_{2}$-NPs can be stored in the dried state and dispersed in the solvent upon ultra-sonication. This reaction can be upscaled up to sub- $1 \mathrm{~g}$ scale and the RAFT-SiO 2 -NPs are storable over months without changing in the performance in polymerization.

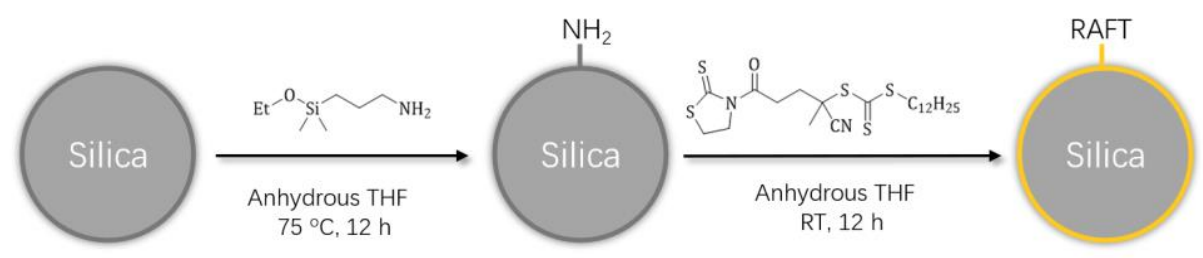

Figure 5.2: Two-step reaction to anchor RAFT group onto the surface of $\mathrm{SiO}_{2}$-NPs.

\subsubsection{Controlled surface-initiated polymerization from silica nanoparticles}

High density polymer can be introduced onto the surface of RAFTfunctionalized $\mathrm{SiO}_{2}$-NPs by simply dispersing the RAFT-SiO 2 -NPs with a polymerization solution containing a predetermined amount of free RAFT agent, monomer, solvent and initiator. The polymerization reaction can 
proceed under conventional condition. Furthermore, the targeted polymer capped $\mathrm{SiO}_{2}$ nanoparticle must be perfectly separated with each other and the spacing between $\mathrm{SiO}_{2}$-NPs must be tunable in well-defined range (10 to $100 \mathrm{~nm}$ ). Come down to synthesize, a suitable solvent, monomer and careful dispersion of RAFT-SiO ${ }_{2}$ are crucial for the successful fabrication of welldefined polymer capped $\mathrm{SiO}_{2}$-NPs.

Here, NiPAAM is chosen for the surface-induced polymerization. The significant advantage brought by the poly(NiPAAM) shell to $\mathrm{SiO}_{2}$-NPs is the excellent dispersibility in different solvent, e.g., ethanol, acetone, chloroform. The wide choice of dispersible solvent is beneficial for optimizing the condition for further treatment i.e. self-assembly with different types of AuNPs(dispersed in water or toluene), dip-coating $\left(\mathrm{CHCl}_{3}\right)$.

One of the most exciting features of the planet-satellite nanostructure is its ability to vary the distance between planet and satellite nanoparticles by changing the chain length of the linking polymer. In doing so, the molecular weight of the grafted polymer on $\mathrm{SiO}_{2}$ must be controlled, i.e., with predeterminable chain length and narrow molecular distribution. The RAFT polymerization (R-approach) gives an easy access to this goal: the chain growth proceeds from the terminal end of the surface-grafted polymer and the RAFT radical attach to the chain-termini reversibly during the polymerization. The molecular weight of the grafted chain is very similar compared with the free RAFT polymer in the solution due to this mechanism. The molecular weight of the yielded polymer is determined by the ratio of RAFT/monomer and polymerization's time. Based on the previous experience, the experimental condition for fabricating RAFT polymer with predetermined molecular weight can be quickly established. A series of experiments was conducted to create polymer capped $\mathrm{SiO}_{2}$-NPs with different chain lengths (Table 5.1). The detailed polymerization conditions are summarized in section 7.4. 
The high density of capped polymer enables the self-assembly of functionalized $\mathrm{SiO}_{2}$-NPs on the substrate into a highly ordered hexagonal nanopattern via simply drop-casting for its dispersion (e.g., ethanol, chloroform). The interparticle distance of resulted hexagonal pattern can be precisely controlled by the length of grafted polymer in the range of 25 to $90 \mathrm{~nm}$ (Figure 5.3). Since these polymers capped $\mathrm{SiO}_{2}$-NPs are used as the nano-planet for the planet-satellite nanostructure, the interparticle distance should logically reach roughly half of the interparticle distance of the pattern created by only nano-planet.

Table 5.1: Results from SEC analysis of the free polymer collected from the synthesis of poly(NiPAAM) capped $\mathrm{SiO}_{2}$-NPs.

\begin{tabular}{lllllll}
\hline Polymer & $\mathrm{A}$ & $\mathrm{B}$ & $\mathrm{C}$ & $\mathrm{D}$ & $\mathrm{E}$ & $\mathrm{F}$ \\
\hline$M_{\mathrm{N} \mathrm{kg}} / \mathrm{mol}$ & 28 & 43 & 53 & 63 & 87 & 270 \\
\hline$D$ & 1.2 & 1.1 & 1.2 & 1.2 & 1.1 & 1.8 \\
\hline
\end{tabular}




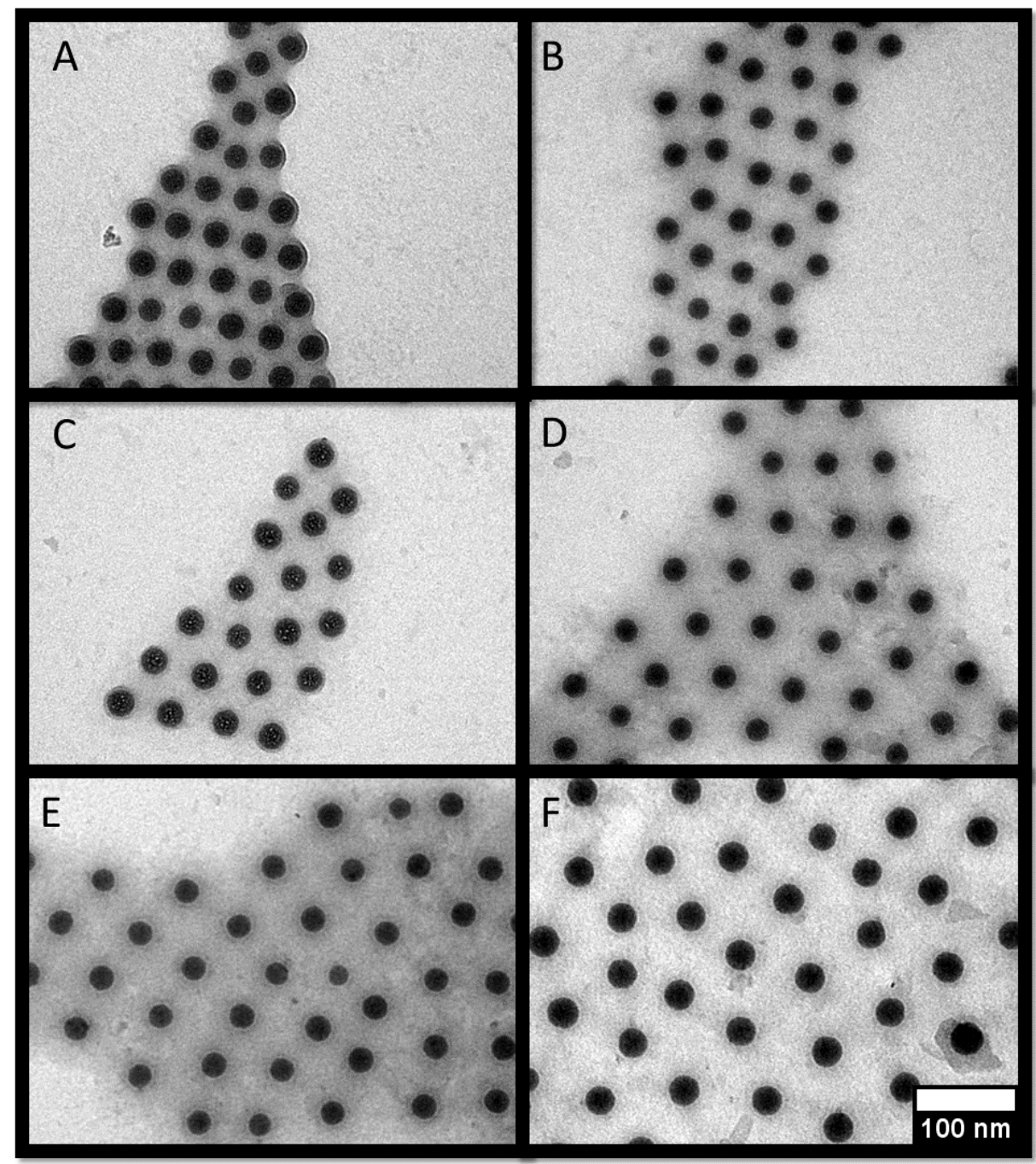

Figure 5.3: TEMicrograph of dense Poly(NiPAAM) capped $\mathrm{SiO}_{2}$-NPs with an increasing chain length of grafted polymer. The increase in interparticle distance can be clearly observed. Sample A-F correspond to the sample listed in Table 5.1. The statistical evaluation of the interparticle distance is presented in Figure 5.4. 


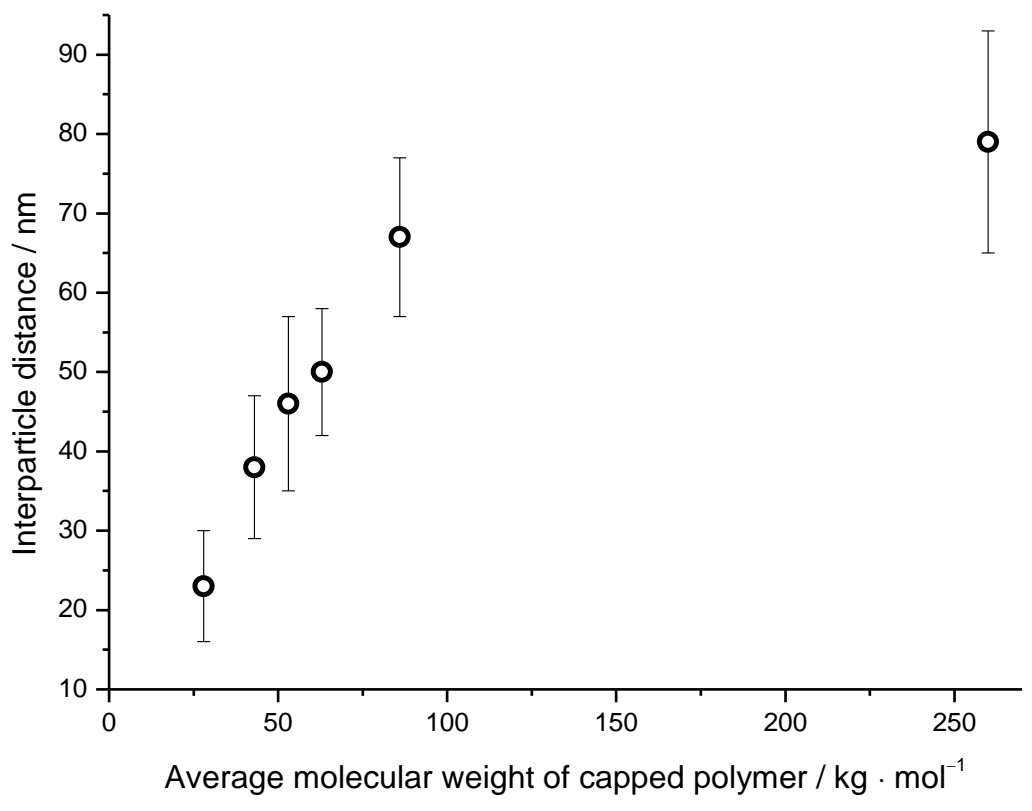

Figure 5.4: Average interparticle spacing in the dependence of the molecular weight of capped polymers for samples listed in Table 5.1.

Beside NiPAAM, other monomers were also tested for the same polymerization's condition. Hydrophilic monomer i.e. PEG-acrylate and PEG-methacrylate were failed to immobilize on the surface of the $\mathrm{SiO}_{2}$ surface, only free-polymer was formed in the solution. The bulk polymerization of RAFT capped $\mathrm{SiO}_{2}$ with MMA was successful with perfectly separated $\mathrm{SiO}_{2}$-NPs and large interparticle distance (Figure 5.5). This MMA capped $\mathrm{SiO}_{2}$-NPs are useful to create planet-satellite nanostructure with hydrophilic dispersibility. 


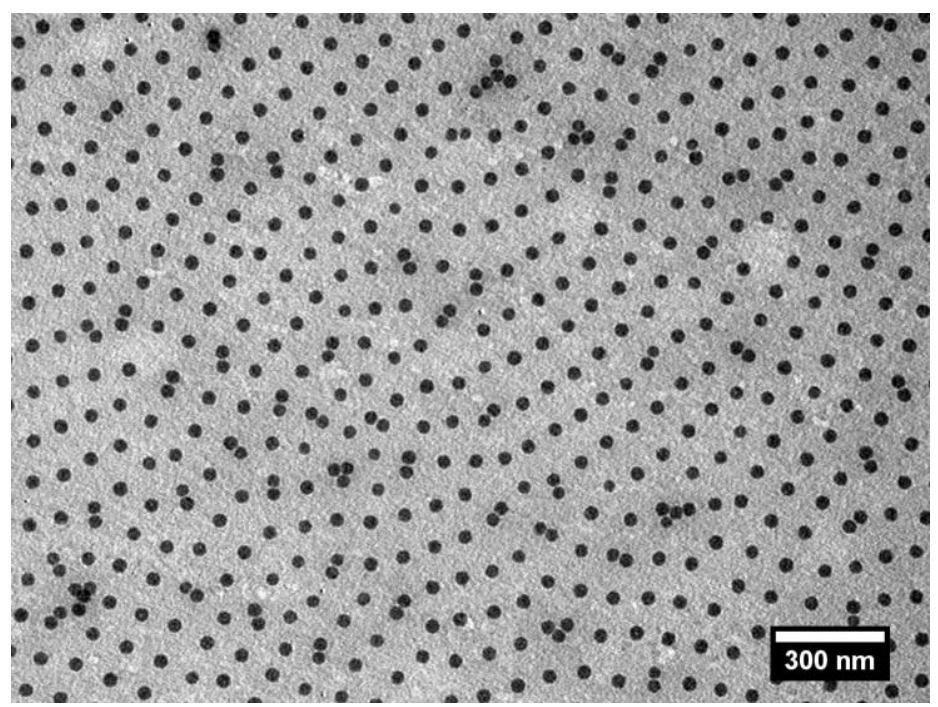

Figure 5.5: TEMicrograph of PMMA capped $\mathrm{SiO}_{2}$-NPs as a monolayer on carbon-film.

\subsection{High-precision self-assembly of silica-planet-gold-} satellite nanostructure

This section focuses on the self-assembly of silica-planet-gold-satellite nanostructures. The logic of optimizing the condition for the colloidal selfassembly process will be discussed in detail. The ability to tune the structure of the binary silica-gold nanostructure will be showcased with the full control of interparticle distance and size of satellite nanoparticles.

The self-assembly of AuNPs onto the planet-structure uses the strong aurophilicity of RAFT moieties on the termini of the polymer grafted on the $\mathrm{SiO}_{2}$-NPs. This diffusion-based procedure requires the simultaneously perfect dispersion of both polymers capped $\mathrm{SiO}_{2}$-NPs and AuNPs in the same solvent. The poly(NiPAAM) capped $\mathrm{SiO}_{2}$-NPs have excellent dispersibility in polar organic solvent, e.g., ethanol, $\mathrm{CHCl}_{3}$. The solvent dispersibility of AuNPs depends, however, on its synthesis and surface protecting ligand. The suitable solvent for the planet and satellite nanoparticles are summarized in Table 5.2. It is worth noting that the $\sim 13 \mathrm{~nm}$ citrate-capped AuNPs are 
synthesized in the water while Brust-Schiffrin AuNPs are obtained in toluene. Drying AuNPs will result in irreversible aggregation. This nature makes the self-assembly of AuNPs with planet nanoparticles in a pure solvent impossible since toluene and water are both bad solvent for poly(NiPAAM) capped $\mathrm{SiO}_{2}$. Mixed solvent with optimized composition must be found for simultaneous dispersion of both planet and satellite nanocomponents.

Table 5.2: Solvent dispersibility of nano components used for self-assembly of silicaplanet-gold-satellite nanostructures.

\begin{tabular}{|c|c|c|c|}
\hline & $\begin{array}{c}\text { poly(NiPAAM) } \\
\text { capped } \mathrm{SiO}_{2} \text {-NPs }\end{array}$ & $\begin{array}{c}\sim 13 \mathrm{~nm} \text { citrate- } \\
\text { capped AuNPs }\end{array}$ & $\begin{array}{c}\sim 5 \mathrm{~nm} \text { TOAB- } \\
\text { capped AuNPs }\end{array}$ \\
\hline $\mathrm{CHCl}_{3}$ & + & - & + \\
\hline $\mathrm{EtOH}$ & + & + & - \\
\hline $\mathrm{H}_{2} \mathrm{O}$ & - & + & - \\
\hline Toluene & - & - & + \\
\hline THF & + & - & - \\
\hline Aceton & + & - & - \\
\hline
\end{tabular}

The logic to find the optimal solvent mixture for both planet and satellite nanoparticles is very straight forward. Since the planet-satellite nanostructure possess the dispersibility comparable to the planetnanocomponent, i.e., poly(NiPAAM), the volume of planet colloid should be much larger than the solvent for AuNPs to prevent any precipitation of the yielded planet-satellite nanostructure. For $\sim 13 \mathrm{~nm}$ citrate-capped AuNPs, a mixture of ethanol and water with a volume ratio of $8: 1$ was used and $\mathrm{CHCl}_{3}$ / toluene mixture with a volume ratio of $10: 1$ was used for Brust-Schiffrin AuNPs.

After mixing the planet and satellite nanoparticles with short sonication treatment or shaking, the colloid is allowed to incubate for self-assembly progress. The diffusion of satellite nanoparticles is much quicker than that of planet nanoparticles due to the much smaller size of satellite nanoparticles. The yielded planet-satellite structure can be stored for 
several hours without any visible aggregation. With the protective linear polymer, the remaining reactive surface of the satellite will be efficiently covered with a linear polymer to prevent further aggregation for longer time (several weeks in ethanol/water mixture and up to a week in $\mathrm{CHCl}_{3}$ ).

Figure 5.6 and Figure 5.8 shows the TEMicrograph of the as-synthesized planet-satellite nanoparticles on carbon substrate with $\sim 5 \mathrm{~nm}$ and $\sim 13 \mathrm{~nm}$ AuNPs, respectively. The sample with $\sim 13 \mathrm{~nm}$ AuNPs as satellites are distributed in a more 2D-fashion compared with the structure using $\sim 5 \mathrm{~nm}$ satellite AuNPs. This difference is possibly caused by the different weights of the satellite AuNPs. Further investigation (e.g., computational simulation) is required to reveal the origin of this difference. However, this intriguing difference in the nanopattern is very important for the application. The "2D" distributed citrate AuNPs is more closed to the desired pattern for the design of biological experiments which focuses on the distance between planet and satellite nanoparticles. This thesis will further focus on the nanostructure with citrate AuNPs as nanosatellites.

It is worth noting that the number of satellite nanoparticles can also be varied by changing the feed ratio of the planet and satellite nanoparticles. Figure 5.7 demonstrates the change of nanostructure while varying the feed ratio of the planet: satellite nanoparticles from $1: 30$ to $1: 200$ for $5 \mathrm{~nm}$ AuNPs as nanosatellite. The number of satellites saturates at approximately 120 satellites per planet nanoparticles for $\sim 5 \mathrm{~nm}$ AuNPs. For $\sim 13 \mathrm{~nm}$ AuNPs as satellites, $1: 20$ was chosen as the exemplary presentation to reduce the complexity of the nanostructure while maintaining the planet-satellite geometry.

Figure 5.8 demonstrates that the distance between the planet and satellite is increasing with the growth of the chain length of linker polymers. The edge-to-edge distance between the planet and the corresponding satellite nanoparticles is statistically evaluated and plot against the average molecular weight of the linker polymer in Figure 5.9. 


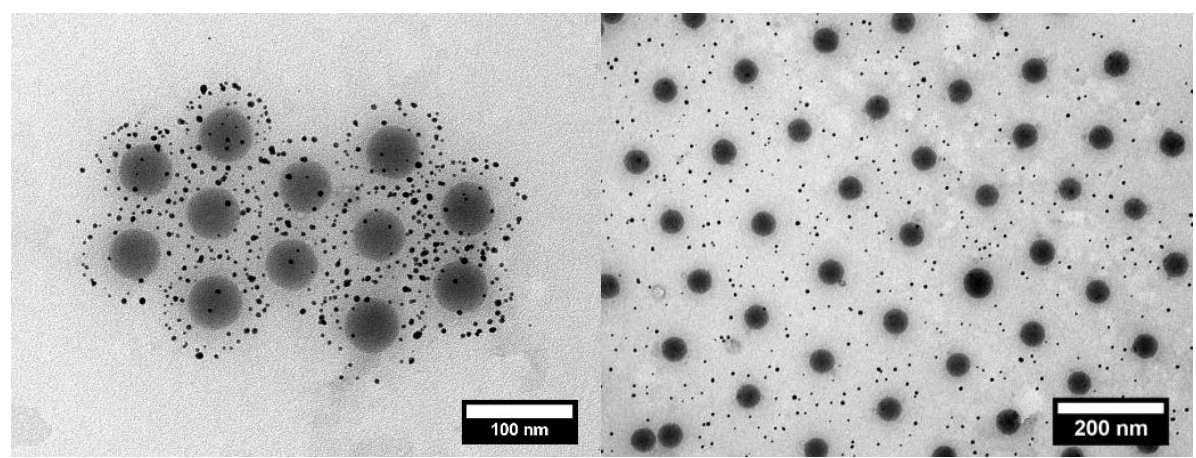

Figure 5.6: TEMicrograph of planet-satellite nanostructure using $\sim 5 \mathrm{~nm}$ AuNPs as satellite nanoparticles. The nanostructure on the left micrograph was using the linker polymer with an average molecular weight of $43 \mathrm{~kg} / \mathrm{mol}$ while the right nanostructure using $87 \mathrm{~kg} / \mathrm{mol}$ linker polymer (Table 5.1).

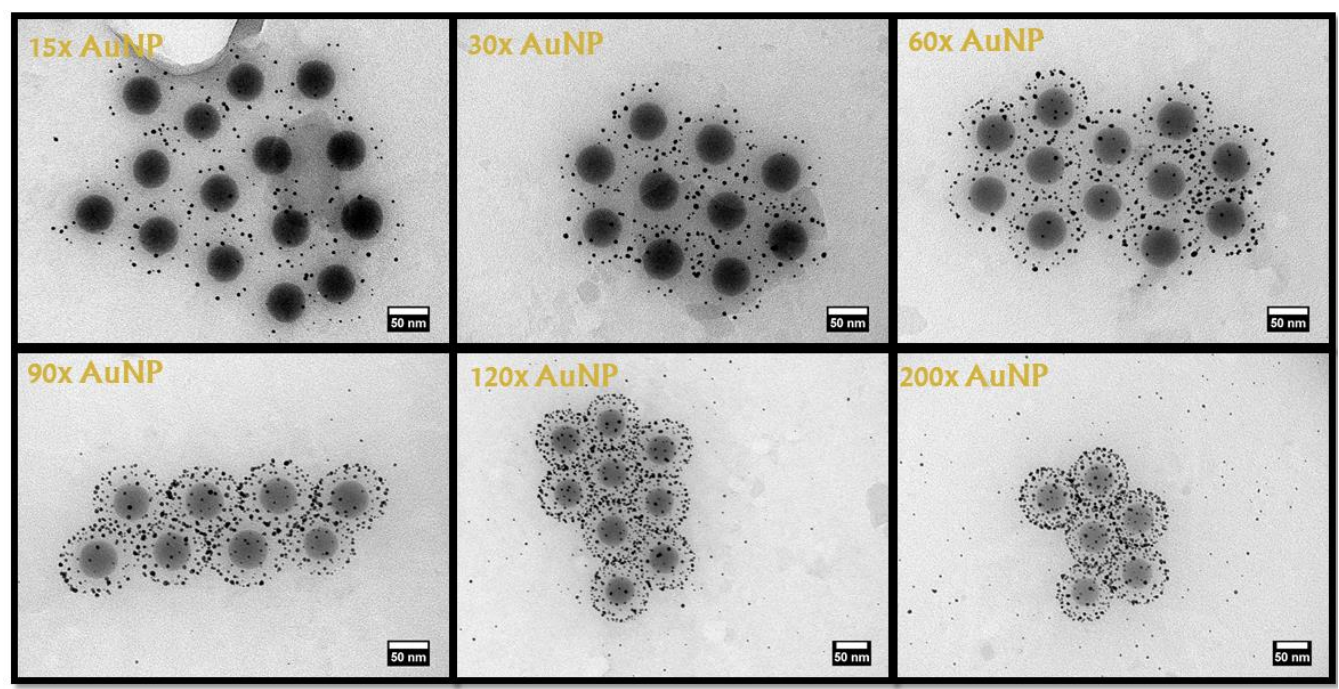

Figure 5.7: Silica-planet-gold-satellite nanostructure using $\sim 5 \mathrm{~nm}$ AuNPs as satellite under different feed ratios of satellite nanoparticles from $1: 15$ to $1: 200$. The excess of AuNPs cannot be completely attached to the planet nanostructure at high dosing of $\operatorname{AuNPs}(>120 \mathrm{x})$. 


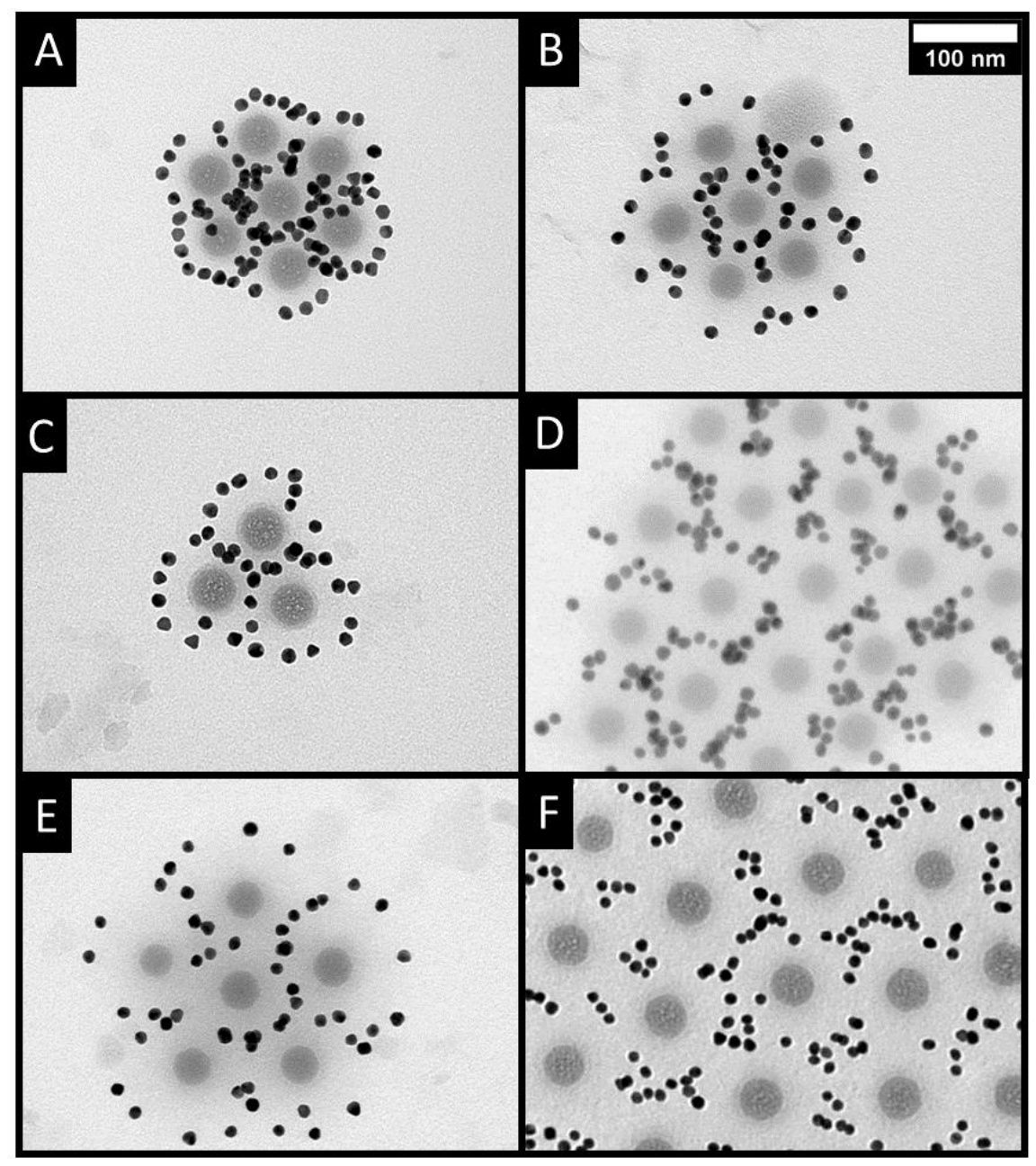

Figure 5.8: TEMicrograph of silica-planet-gold-satellite nanostructures using $\sim 13 \mathrm{~nm}$ AuNPs as nanosatellites with feed ratio of $\mathrm{SiO}_{2}$-NPs / AuNPs $=1: 20$. Sample A-F correspond poly(NiPAAM) capped $\mathrm{SiO}_{2}$-NPs listed in Table 7.1. The statistical evaluation of the interparticle distance is presented in Figure 5.9. 


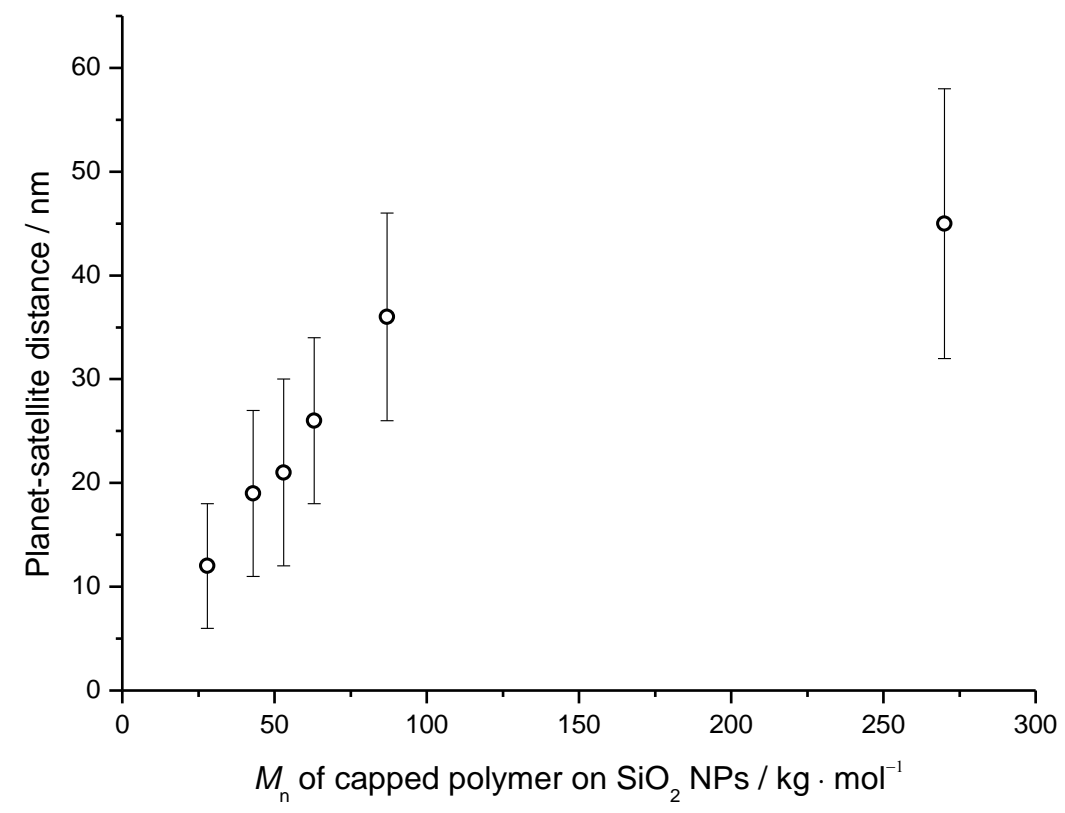

Figure 5.9: Interparticle distance between a planet ( $\mathrm{SiO}_{2}$-NPs) and satellite (AuNPs) in the dependence of molecular weight of linker polymer on the poly(NiPAAM) capped $\mathrm{SiO}_{2}$-NPs.

It can be seen from Figure 5.9 that this approach enables the control of the planet-satellite distance between 10 and $50 \mathrm{~nm}$ with high precision. The distance-dependent cell response studies require a high precision of nanopattern with the well-defined relationship between nanocomponents with interparticle distance in sub-100 $\mathrm{nm}$ range. Here, the 2D distribution of satellite and the range of interparticle distance meet well with the geometrical requirement from the biological cell response experiment.

The next step is to create a homogenous monolayer of the planetsatellite nanostructure on a substrate rather than the island-like random distribution. 


\subsection{D nano-patterning with the planet-satellite}

\section{nanostructure}

The cell response experiments based on patterned nanoparticle must be carried out on a transparent substrate. This brings the first task to find a suitable substrate providing a clean background for the experiment, i.e., free of any bio active molecules/moieties after all functionalization steps. In the previous works, PEGylated glass and PEG matrix were used to serve this role. However, due to the similarity of glass and silica, the glass cannot be applied her. The binary silica-planet-gold-satellite nanopattern has higher requirement on the substance. The substrate should simultaneously possess the following properties: transparent, nano-flat, biological neutral, good solvent tolerance, chemically distinct with gold and silica and stable for plasma treatment. $\mathrm{CaF}_{2}$ quickly becomes one of the best choices considering all these demands. A detailed discussion on choosing a suitable substrate can be found in section 3.2.2.

To create a nanostructured surface $\left(\mathrm{CaF}_{2}\right)$, a macroscopic homogenous monolayer of the planet-satellite nanostructure needs to be formed. There are several potential techniques, e.g., dip-coating, spin-coating. Here, the dip-coating technique is chosen for the presence of the dipping-line which can be used as a convenient comparing sample in the cell-response experiment. However, a complex variety of parameters are present for optimizing the formation of a homogenous monolayer of nanoparticles on substrates. Thus, a number of experiments are performed here systematically. 


\subsubsection{Monolayer formation of planet-satellite nanostructure on the surface via dip-coating}

The dip-coating method is a very straightforward technique to create thin-film on a surface by pulling the substrate from the colloid/solution at certain speed. For the planet-satellite nanostructure, there are numerous parameters affecting the formation of the monolayer of the nanoassemblies: the moving velocity of the substrate, evaporation speed, contact angle between the liquid and the substrate, density of the colloid, the concentration of the colloid, angle of the substrate, temperature and pretreatment of the substrate. Simplification is crucial here for effective optimization of the dip-coating process. The temperature is set at room temperature (in an air-conditioned room at $23^{\circ} \mathrm{C}$ ). The angle between the substrate and surface of the liquid stays perpendicular. All substrates have first washed with pure ethanol and dry with argon flow then treated with plasma $(100 \mathrm{~W}, 40 \mathrm{k} \mathrm{Hz})$ for $10 \mathrm{~min}$ before coating. Only two practical parameters are now varied here: type of solvent and pulling velocity.

\section{Hardware for dip-coating}

The dip-coating process is conducted using a self-designed dip-coater (Figure 5.10A) crafted by the workshop for the institute for physical chemistry at the University of Göttingen. The vertical moving arm is driven by a stepper motor. The speed of the stepper motor is digitally controlled by a program with a graphic user interface. The substrate is clamped with a reverse tweezer with a piece of a strong magnet attached at the bottom (Figure 5.10B). The reverse tweezer is then fixed on the vertical moving arm (on another magnet) and stays vertical during the whole operation (Figure 5.10C). It is worth mentioning that the tip of the reverse tweezer is wrapped with PTFE tape in order to increase the contact area between substrate and tweezer making the clamping more stable. The velocity of the vertical moving arm can be set from $0.05 \mathrm{~mm} / \mathrm{min}$ to $10 \mathrm{~mm} / \mathrm{min}$ in the 
step of $0.05 \mathrm{~mm} / \mathrm{min}$. This range sufficiently covers the typical condition for dip-coating.

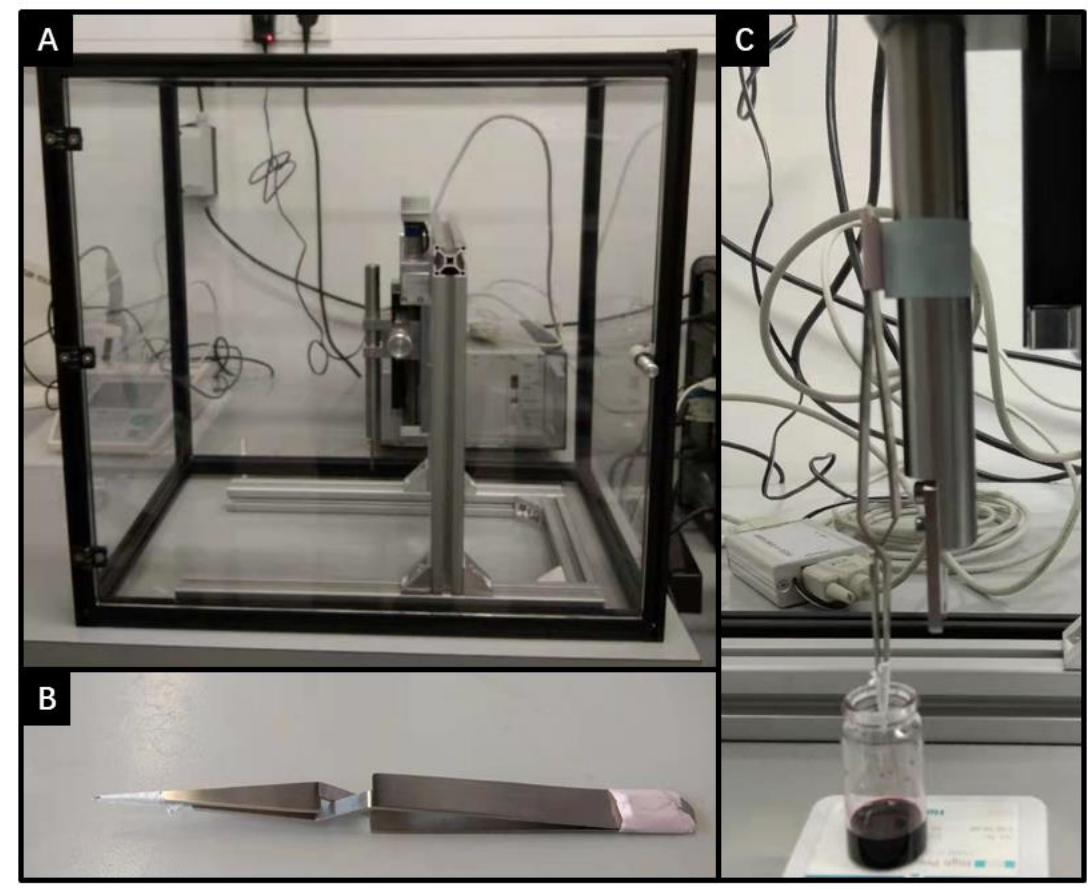

Figure 5.10: (A) Mechanical part of the dip-coater installed in a windproof cabinet. (B) Reverse tweezer used for clamping the substrate. The tip is wrapped with PTFE tape and a piece of a strong magnet is attached to the bottom of the tweezer. (C) Dip-coater in operation.

\section{Preparation of concentrated collide for dip-coating}

The preparation of colloid containing perfectly dispersed planetsatellite nanostructure. However, the silica-planet-gold-satellite nanostructure is dispersed in ethanol/water mixture with a very low concentration (appx. $0.05 \mathrm{mg} / \mathrm{ml}$ ) after the synthesis. The typical concentration for dip-coating using polymer capped AuNPs should be at $1 \mathrm{mg} / \mathrm{ml}\left[{ }^{98]}\right.$. One of the major challenge here is the concentrating of the nanostructure colloid, including solvent exchange from ethanol/water mixture to the desired solvent. Centrifugation is highly efficient to gather all the nanoparticle and remove all the solvent here. By aggressive 
centrifugation of planet-satellite nanostructure by $8000 \mathrm{rpm}$ for $2 \mathrm{~h}$, the nanostructure can be completely gathered as sediment material at the bottom of the centrifuge tube. However, the centrifugation of nanostructures often results in an irreversible aggregation which means the gathered nanostructures will not be able to fully dispersed into any solvent even after long $(>1 \mathrm{~h})$ ultrasonication. Based on the experience, this issue is caused by the lengthy and strength of the centrifugation. Meanwhile, this harsh centrifugation condition is necessary to gather the nanostructure from the colloid effectively.

In order to moderate the centrifugation condition, change of the solvent by adding poor solvent for the nanoassemblies is generally feasible. The idea is to decrease the dispersibility of the planet-satellite nanostructure while maintaining the solubility of excess linear polymer (excess polymer must be removed via centrifugation) by adding further solvent into the assynthesized colloid. A typical experiment yield $90 \mathrm{ml}$ as-synthesized colloid (80 ml ethanol, $10 \mathrm{ml}$ water) consists of ca. $10 \mathrm{mg}$ perfectly dispersed planet-satellite nanostructure and $1 \mathrm{mg}$ of the excess linear polymer. The linear polymer carries decent water solubility (ca. $1 \mathrm{mg} / \mathrm{ml}$ in pure water) while the planet-satellite nanostructure cannot be dispersed in pure water. Adding further water to the ethanol/water mixture can gradually decrease the dispersibility of the planet-satellite nanostructure without precipitation of linear polymer. The sweet spot of the amount of additional water was found to be approximately $120 \mathrm{ml}$ pure water to $90 \mathrm{ml}$ as-synthesized colloid (water : ethanol = $20: 1$ ). Under this condition, the planet-satellite nanostructure can be easily gathered under only 20 minutes using $6000 \mathrm{rpm}$. The planet-satellite nanoparticles are gathered on the wall as a thin film (rather than a tiny spot, Figure 5.11) due to the quick sedimentation during the centrifugation process. This film of the planet-satellite nanostructure can be completely redispersed in $\mathrm{CHCl}_{3}$ with the aid of bath ultrasonication under 10 minutes. Detailed procedure of this solvent exchange process can be found in experimental section. 
Owing to the highly efficient centrifugation process, all the planetsatellite nanoparticles can be collected. In this way, the concentration of planet-satellite nanostructure can be estimated by assuming that all the nanoassemblies are collected and dispersed in the final dip-coating colloid.

Using this method, the planet-satellite nanoassemblies are able to be dispersed in any suitable solvent with predeterminable concentration for the successive coating process.

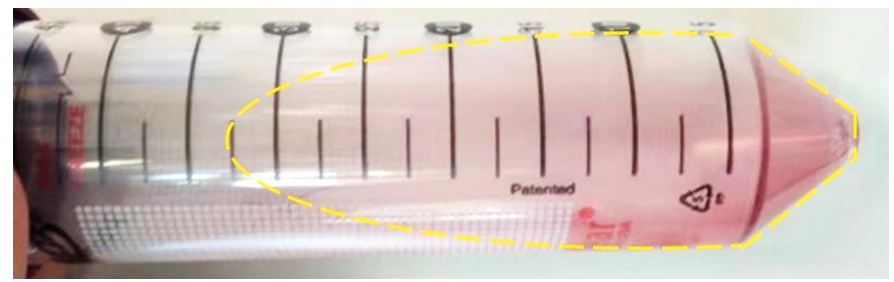

Figure 5.11: Film-like of silica-planet-gold-satellite nanostructures collected by the centrifugation process (6000 rpm, $20 \mathrm{~min}$ ) with the aid of additional water in the colloid. Yellow lines highlight the area of collected nanoassemblies.

\section{Optimizing dip-coating parameters}

As mentioned in section 5.4.1, the number of parameters for optimizing dip-coating is reduced to only the type of solvent and the dipping velocity. In this thesis, ethanol and chloroform were tested for dip-coating. As a result, chloroform is found to be a suitable solvent for the monolayer formation of silica-planet-gold-satellite nanoassemblies. The dip-coating experiments were conducted on the glass, silicon wafer and $\mathrm{CaF}_{2}$ crystal slices. All surfaces are highly polished with roughness $<5 \mathrm{~nm}$ (confirmed by AFM).

For all dip-coating experiment, the substrate was first washed with clean ethanol and dried with argon flow. After 10 minutes plasma treatment, the substrate was immediately taken to the dip-coating process. After coating, the samples are analyzed with AFM to obtain the quality of the monolayer formation. Typically, there are the following situations: (a) Only islands of nanoparticles are formed, with no large area $(>\mu \mathrm{m})$ completely covered by the nanoassemblies. (b) Monolayer formation is completed over the whole 
sample while significant areas without nanoassemblies are present on the surface. (c) Homogenous monolayer is formed over the whole substrate, which is the desired situation. (d) Double layer or multilayer of the nanoassemblies are formed on the top of the monolayer. In general, insufficient concentration and high velocity of the dip-coating cause the incomplete formation of the monolayer, while excessive concentration causes the occurrence of multilayer issue. In a specific range of concentration and pulling velocity, the homogenous monolayer formation can be achieved in a highly reproducible manner. Figure 5.12 shows several AFM graphs of the typical situations mentioned above. Table 5.3 summarize the results from the dip-coating experiment.

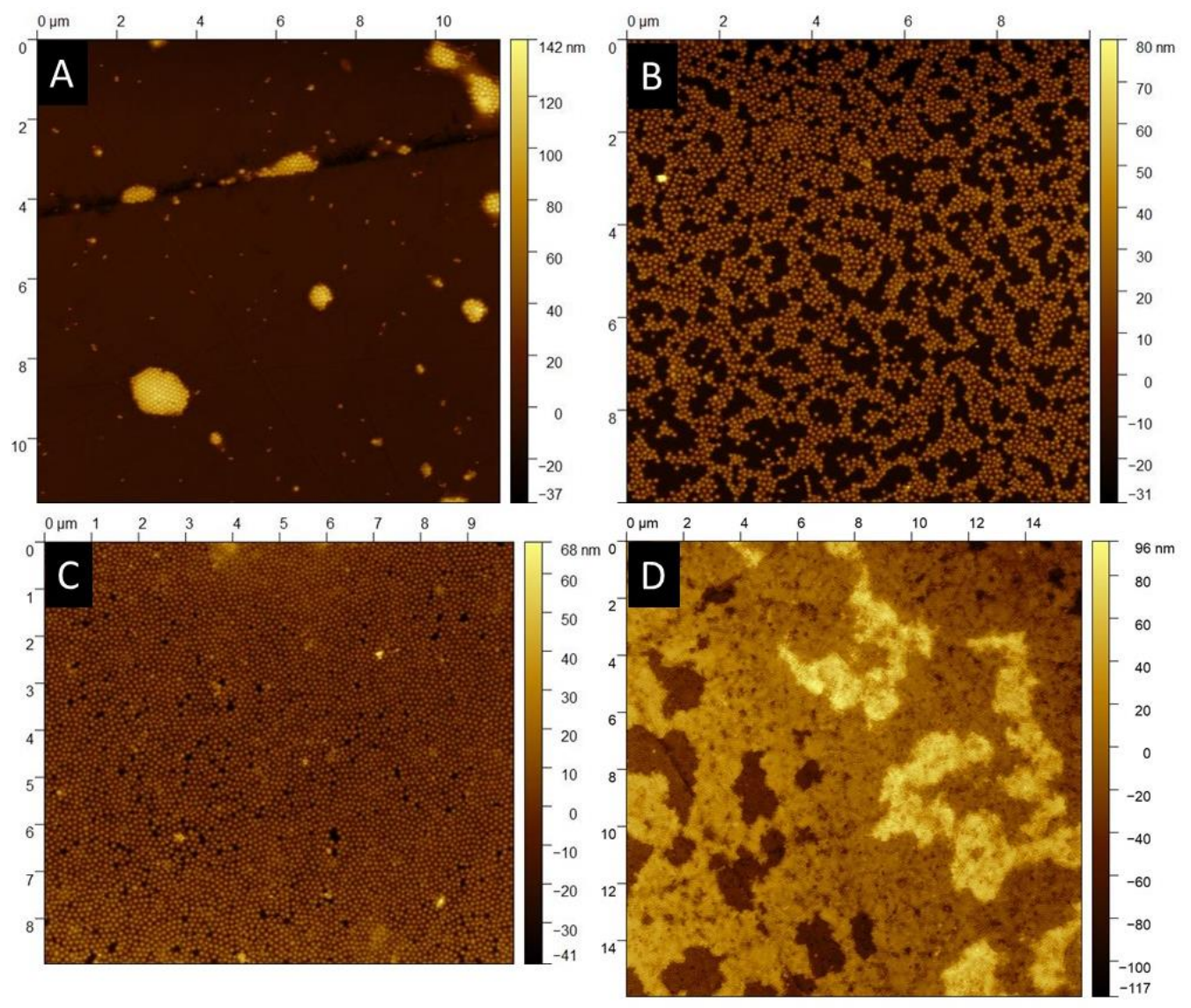


Figure 5.12: Typical results for dip-coating from AFM analysis. (a) Island-like, (b) Monolayer with holes, (c) homogenous monolayer and (d) multilayers (The brown colored area is the first layer, the dark-yellow area is the $2^{\text {nd }}$ layer and the light-yellow area is the $3^{\text {rd }}$ area). 
Table 5.3: Summarized results from dip-coating under different pulling velocity using ethanol and chloroform as solvent for silica-planet-gold-satellite nanostructure. $(\triangle)$ Island-like, ( $(\circ)$ monolayer with holes, $(\mathbf{O})$ homogenous monolayer and ( $\square$ ) multilayers

\begin{tabular}{|c|l|l|l|l|l|l|}
\hline $\begin{array}{l}\text { Pulling } \\
\text { velocity } \\
\mathrm{mm} / \mathrm{min}\end{array}$ & $\begin{array}{l}\text { Ethanol } \\
1 \mathrm{mg} / \\
\mathrm{ml}\end{array}$ & $\begin{array}{l}\text { Ethanol } \\
2 \mathrm{mg} / \\
\mathrm{ml}\end{array}$ & $\begin{array}{l}\text { Ethanol } \\
4 \mathrm{mg} / \\
\mathrm{ml}\end{array}$ & $\begin{array}{l}\mathrm{CHCl}_{3} \\
1 \mathrm{mg} \\
/ \mathrm{ml}\end{array}$ & $\begin{array}{l}\mathrm{CHCl}_{3} \\
2 \mathrm{mg} \\
/ \mathrm{ml}\end{array}$ & $\begin{array}{l}\mathrm{CHCl}_{3} \\
4 \mathrm{mg} \\
/ \mathrm{ml}\end{array}$ \\
\hline 8 & & & & & & $\triangle$ \\
\hline 4 & & & & & $\bigcirc$ & $\bigcirc$ \\
\hline 2 & & $\triangle$ & $\triangle$ & $\triangle$ & $\bigcirc$ & $\bigcirc$ \\
\hline 1 & $\triangle$ & $\triangle$ & $\triangle$ & $\triangle$ & $\bigcirc$ & \\
\hline 0.6 & $\triangle$ & $\triangle$ & $\triangle$ & $\triangle$ & $\bigcirc$ & $\square$ \\
\hline 0.4 & $\triangle$ & $\triangle$ & & \\
\hline
\end{tabular}

Based on the results from Table 5.3, chloroform was proved to be a suitable solvent for the dip-coating of silica-planet-gold-satellite nanoassemblies. In the range of 2 to $4 \mathrm{mg} / \mathrm{ml}$ and pulling velocity in 1 to $0.4 \mathrm{~mm} / \mathrm{ml}$ the monolayer formation can be archived with high reproducibility as evidenced by SEM (Figure 5.13). Furthermore, no significant difference was found between glass, silicon wafer and $\mathrm{CaF}_{2}$ substrate under the same dip-coating condition. Also, no significant impact of chain-length dependence of the polymer grafted to the $\mathrm{SiO}_{2}$-NP surface was observed for the performance of the dip-coating process. 


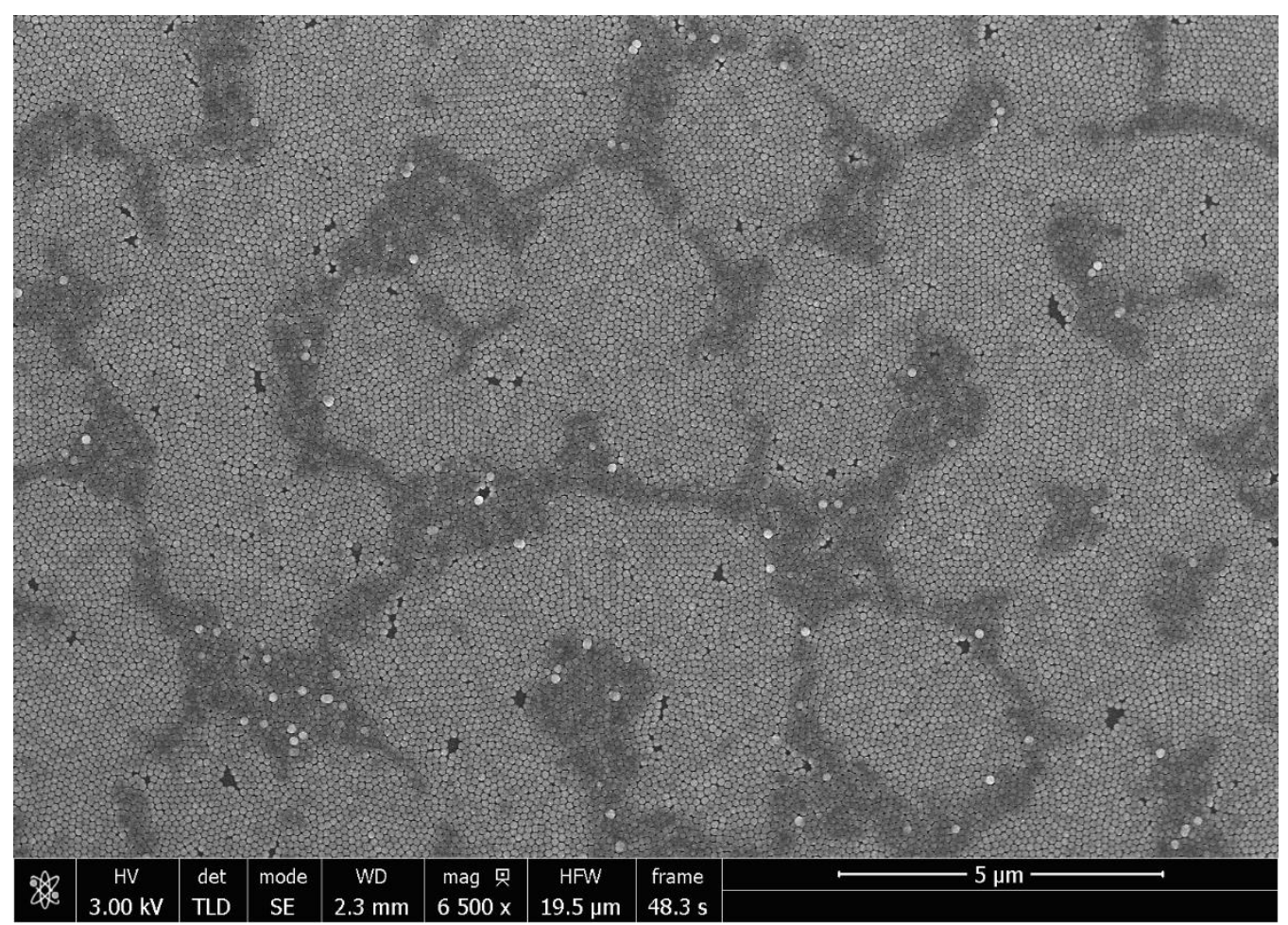

Figure 5.13: SEMicrograph of the monolayer of the planet-satellite structure $\mathrm{F}$ on a silicon wafer. Each spherical object corresponds to a single planet-satellite nano assembly.

Using the dip-coating method, a well-defined dipping line can be easily archived. The two sides of the dipping front can serve as perfect intern comparing samples (Figure 5.14). 
Figure 5.14: A sample of $\mathrm{CaF}_{2}$ substrate coated with the plant-satellite nanostructure. The reddish-pink area is decorated with a monolayer of planet-satellite nanoassemblies.

The samples carrying only patterned AuNPs and $\mathrm{SiO}_{2}$-NPs are also necessary for the control experiment in biological studies. Under the same optimized condition, the monolayer formation of poly(NiPAAM) capped AuNPs, and $\mathrm{SiO}_{2}$-NPs were also successful.

\subsubsection{Removal of polymer content by plasma treatment}

In order to expose the original surface of AuNPs and $\mathrm{SiO}_{2}$-NPs for further biological functionalization, the polymer content on the nanoparticles must be completely removed without any residue. Oxygen plasma has been reported to be a very reliable and efficient method for this task (section 3.2). Here, the relative mild plasma condition was chosen for the planet-satellite nanostructure to avoid any melting of AuNPs due to the rise of the temperature. Briefly, the substrate carrying monolayer of the polymerbased planet-satellite sample is placed in the plasma chamber. With a power of $100 \mathrm{~W}$, the plasma treatment proceeds for 2 min with 1 min pause for five cycles. The plasma-cleaned sample remains the same color (reddish) indicating no melting (geometry change) or aggregation of AuNPs during the plasma process. 
AFM measurement was also conducted for the plasma-treated substrate. For polymer-based planet-satellite nanostructure sample $F$, the height of nanostructure reduced from $\sim 90 \mathrm{~nm}$ to $\sim 35 \mathrm{~nm}$ after plasma treatment (Figure 5.15). The $\mathrm{SiO}_{2}$-NPs used in these nanoassemblies have a diameter of $35 \mathrm{~nm}$ which matches perfectly with the height profile after plasma treatment indicating no measurable residual polymer content. The texture of nanoparticles from AFM capture also changed after plasma treatment due to possibly the insufficient adhesion between nanoparticles and substrate.

It is worth to mention that only the upper side of the substrate is used for further applications, although no difference in the performance between both sides is observed.

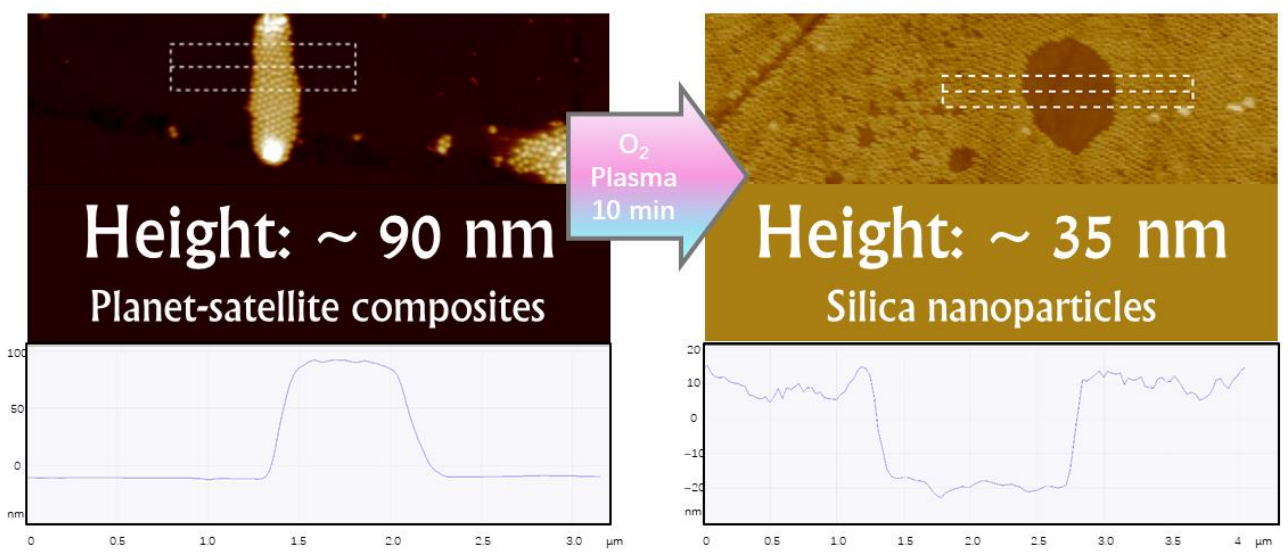

Figure 5.15: Comparison of height profile of AFM captures of a monolayer of the polymer-based planet-satellite nanostructure (sample F) on silicon wafer before and after plasma treatment. The height is evaluated using the data from the marked windows statistically and displayed below the AFM capture. The significant reduction of height from $\sim 90 \mathrm{~nm}$ to $\sim 35 \mathrm{~nm}$ indicates the complete removal of polymer content since $35 \mathrm{~nm}$ corresponds exactly to the size of synthesized $\mathrm{SiO}_{2}$-NPs. A change in the AFM texture can also be observed due to the changed interaction between nanoparticles and substrate.

It is concerned that the insufficient plasma etching of polymer content might generate a trace of carbon-based contaminations on the surface of nanoparticles. Previous work ${ }^{[102]}$ has confirmed that the carbon content of 
polymer micelle loaded AuNPs can be completely removed using plasma treatment (both hydrogen and oxygen). It is worth noting that the thickness of the polymer layer in that work and the polymer shell from the silicaplanet-gold-satellite nanostructure are very comparable $(<100 \mathrm{~nm})$. In this context, the plasma treatment should be suitable to expose the surface of both AuNPs and $\mathrm{SiO}_{2}$-NPs for further functionalization at least for this type of application.

\subsubsection{Transfer of binary planet-satellite nanopattern onto the hydrogel}

The hydrogel is a very widely used material for biological experiments. In the realm of nanopattern induced cell response experiment, hydrogel (cross-linked PEG) is generally the ideal substrate for the experiments for its passive biological activity and soft mechanical properties. The biological inertness provides a perfect background with no presence of biological labels (e.g., protein, peptide). The softer mechanical property of hydrogel can mimic the property of the cell environment more realistically.

The dip-coating cannot proceed on the surface of hydrogel due to its water content. The plasma treatment to remove the polymer content of the planet-satellite assemblies can also simultaneously activate the hydrogel (oxidation induced - $\mathrm{OH}$ and - $\mathrm{COOH}$ groups) which reduce the bio-inertness of PEG causing unspecific adsorption of biomolecules on the hydrogel. The planet-satellite nanoassemblies must be created on the inorganic substrate, i.e., $\mathrm{CaF}_{2}$ in the first step.

Although carrying no -OH group, $\mathrm{CaF}_{2}$ is not suitable for cell experiments without any passivation. On this point, PEGylation is the commonly used method to archive the biological passivation on substrate like glass. In section 5.4.3, PEGylation on the $\mathrm{CaF}_{2}$ surface is attempted using a protocol that has been successful applied on the glass surface. It is confirmed that the 
PEG passivation is sufficient for melanoma cells but inadequate for an RGD peptide. The unspecific adsorption of RGD peptide on the background strongly jeopardizes the application by introducing bio-label onto the background. Transferring the nanopattern onto hydrogel in high fidelity is crucial to push the silica-gold binary nanopattern to its desired level of application.

Hence, a line of the experiment is designed for transferring both AuNPs and $\mathrm{SiO}_{2}$-NPs entirely onto the surface of the hydrogel. In brief, after removing all polymer content on the monolayer of the planet-satellite nanostructure decorated $\mathrm{CaF}_{2}$ surface, linker molecules (acylate) for AuNPs and $\mathrm{SiO}_{2}$-NPs are introduced to the surface of nanoparticles. With the aid of the linker, both types of nanoparticles are able to transfer onto the hydrogel by photopolymerization PEG-diacrylate with the $\mathrm{CaF}_{2}$ substrate.

\section{The attempts of surface passivation of $\mathrm{CaF}_{2}$ by PEGylation}

To evaluate the potential application of the binary AuNPs and $\mathrm{SiO}_{2}$-NPs decorated $\mathrm{CaF}_{2}$ for the cell-responsive experiment, the biological inertness of background (i.e., $\mathrm{CaF}_{2}$ substrate) must be studied first. In the early works, glass was chosen as the substrate. Due to the -OH group on the glass' surface, the substrate has a strong affinity towards cells and biological molecules (e.g., protein, peptide). The strong bio-affinity will critically jeopardize the cell-response experiment: cells will attach to the surface and even deform (e.g., adhesion) itself on the substrate; biological labels will be unspecifically functionalized over the whole substrate making the nano-pattern lose its role for being the template for the corresponding bio-labels.

In this regard, the interaction between $\mathrm{CaF}_{2}$ and cell/bio-labels must be studied in the first place. The inertness of the background must be proved in order to get any rational conclusion for any biological experiment. So far, the biological affinity of $\mathrm{CaF}_{2}$ for the cell-response experiment have not been reported in the literature. On this point, the attachment of melanoma cell 
and cyclic RGD on the $\mathrm{CaF}_{2}$ surface is tested. The melanoma cell and RGD peptide are the candidates for the cell-response experiments on the binary pattern. In brief, the RGD peptide is known to induce the cell spreading/adhesion for melanoma cells if the distance between RGD is suitable.[137] Melanoma cell is the cause of many skin cancers and being focused by our cooperation partner.

The test of bio-affinity can be carried out in a simple experiment. By incubating the substrate with a solution containing a certain amount of melanoma cells and then washing with phosphate-buffered saline (PBS), the bio-affinity of the surface can be directly evaluated through observing the behavior of the cells. For a passive surface, most cells will be washed off from the surface after incubation and the remaining cells stay spherical (Figure 5.16a). For an active surface, the cells tend to attach to the surface firmly, the washing process will not significantly decrease the number of attached cells on the substrate (Figure 5.16b). From this test, $\mathrm{CaF}_{2}$ showed a strong affinity towards melanoma cells. In this context, the surface passivation (PEGylation) on $\mathrm{CaF}_{2}$ is inevitable for further experiments.

The PEGylation on $\mathrm{CaF}_{2}$ seems a challenging for the lack of known chemistry on $\mathrm{CaF}_{2}$ crystals. However, the widely used PEGylation on glass by using poly-l-lysine-graft-peg (PLL-g-PEG) is based on the electrostatic interaction ${ }^{[138]}$ between positively charged PLL chain (protonated $-\mathrm{NH}_{2}$ group) and negatively charged glass surface (deprotonated -OH group). In this regard, the surface passivation of the $\mathrm{CaF}_{2}$ using PLL-g-PEG is carried out using the same protocol by introducing charges using oxygen plasma. The PEGylation was carried out by simply incubating the plasma-treated $\mathrm{CaF}_{2}$ in a $1 \mathrm{mg} / \mathrm{ml}$ PLL-g-PEG solution for $40 \mathrm{~min}$. The sample is then washed with water and dried with argon flow. The freshly prepared sample was incubated in melanoma cells culture medium and then rinsed with water. 

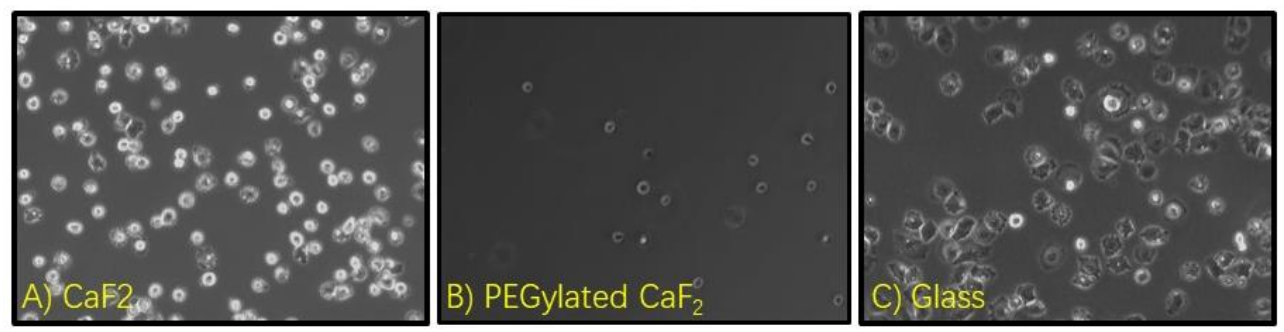

Figure 5.16: Micrograph of remaining melanoma cells on the substrate after incubation and rinsing with water. A) Plasma treated $\mathrm{CaF}_{2}$ surface. A significant amount of cells are attached to the surface while maintaining its spherical shape. B) PEGylated $\mathrm{CaF}_{2}$ surface. The number of attached cells is significantly reduced compared with A). C) Plasma treated glass surface without passivation. Cell spreading can be observed here indicating a strong interaction between unprotected glass surfaces and cells.

Figure 5.16 demonstrates the results of the cell's attachment test on original $\mathrm{CaF}_{2}$, PEGylated $\mathrm{CaF}_{2}$, and glass (control sample), respectively. The effective passivation of PLL-g-PEG on $\mathrm{CaF}_{2}$ is confirmed by observing a significantly decreased number of attached melanoma cells. Furthermore, no cell deformation was observed on $\mathrm{a} \mathrm{CaF}_{2}$ substrate indicating that no strong biological activity is taking place between $\mathrm{CaF}_{2}$ substrate and cells.

The next step is to validate if the passivation of $\mathrm{CaF}_{2}$ is also sufficient against the biomolecules. A thiolated (by cysteine) cyclic RGD (cyclo(RGDfE)-PEG-5-CCC) is used here which is design for the functionalization on AuNPs. Briefly, after the polymer content of the planetsatellite nanostructure decorated $\mathrm{CaF}_{2}$ substrate is removed by plasma treatment, the sample is first incubated in PLL-g-PEG solution for PEGylation. After rinsing with water, the substrate is then placed on a droplet of RGD solution for 3 hours for a sufficient immobilization of the biolabels on AuNPs. The same procedure of cell response is then repeated.

Figure 5.17 shows the result of the cell response experiment. The response of melanoma cells can be clearly observed from B) and C), indicating the presence of RGD peptide on the surface. However, the cell spreading can be observed on both sides (left $=$ with nanoparticles, right $=$ 
blank) of the dipping line on $\mathrm{C}$ ), indicating the non-specific binding of RGD on the $\mathrm{CaF}_{2}$ background. This non-specific binding of biomolecules is unfavored for the cell-response experiment. In this regard, the present method of using PLL-g-PEG on of $\mathrm{CaF}_{2}$ provides only limited passivation which is sufficient for melanoma cell but not for RGD peptides.
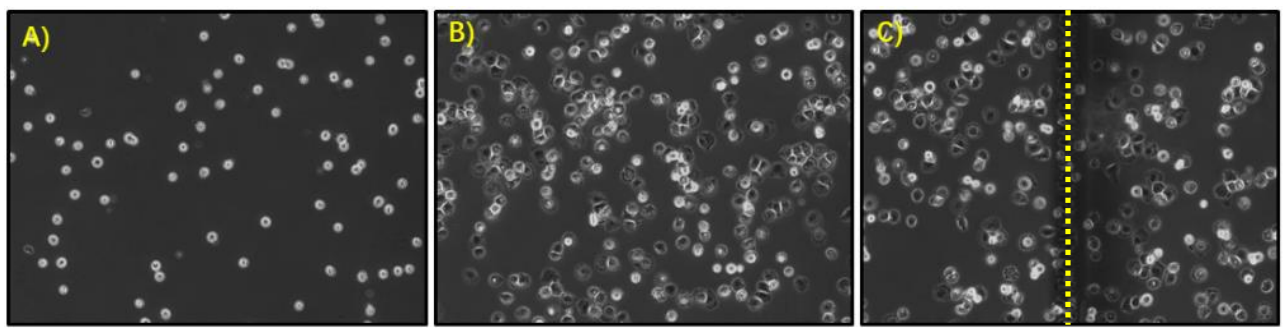

Figure 5.17: Micrograph of remaining melanoma cells on the substrate after incubation with melanoma cells and rinsing with water. A) On the PEGylated $\mathrm{CaF}_{2}$ surface without nanoparticles and RGD peptide. B) PEGylated $\mathrm{CaF}_{2}$ surface which has incubated with RGD solution. The number of attached cells is significantly raised (compared with A) with significant cell spreading. C) Binary planet-satellite nanoparticles decorated $\mathrm{CaF}_{2}$ substrate with PEGylation and RGD. The yellow line in the middle of the micrograph is the dipping-line. The left-side of the graph is decorated with nanoparticles while the right-side contains no NPs.

Since the passivation meets its bottleneck on $\mathrm{CaF}_{2}$, the strategy to develop a new approach is required here for providing an inert background for both cells and biomolecules. In the next section, a method for transferring the nanopattern onto hydrogel will be demonstrated for this task.

\section{Transferring binary nanopattern from $\mathrm{CaF}_{2}$ onto hydrogel}

To transfer patterned nanostructures from inorganic substrate onto hydrogel requires a linker molecule which covalently connecting NPs and hydrogel matrix (section 3.2.1). For transferring AuNPs from glass to hydrogel, N,N'-Bis(acryloyl)cystamine (BAC) was used in previous works. ${ }^{[69,77]}$ BAC contains a disulfide group that is strongly aurophilic thus 
provides a very stable covalently binding to AuNPs while the acryloyl endgroups of BAC also provide covalent bond to hydrogel (PEG-diacrylate) matrix during the photon-initiated polymerization.

For $\mathrm{SiO}_{2}$-NPs, a suitable linker must be chosen and the condition for its anchoring on NPs must be optimized to provide sufficient capping density for the transfer. Again, the silane chemistry can be applied here: the linker molecule should carry silyl ether moiety for anchoring a $\mathrm{SiO}_{2}$ surface while possesses the acryloyl group for binding into the hydrogel matrix. 3Acryloxypropyltrimethoxysilane (APTOS) is chosen as a linker here. Base on the experience, the silane anchoring experiment can proceed well in a polar solvent (e.g., $\mathrm{CHCl}_{3}$ or THF) at room temperature. $\mathrm{CHCl}_{3}$ is chosen here since both BAC and APTOS are soluble in it. Practically this means both linker molecules can be introduced onto the corresponding NPs' surfaces in a single step. The process of the transfer is illustrated in Figure 5.18. The substrate is incubated in a $\mathrm{CHCl}_{3}$ solution containing $1 \mathrm{mM} \mathrm{BAC}$ and $100 \mathrm{mM}$ APTOS overnight at room temperature. After rinsing with clean $\mathrm{CHCl}_{3}$, the sample is ready for the transfer.

The transfer step is very staightforward: by polymerizing PEG-diacrylate with the prepared sample, the nanoparticles will be covalently bound into the cross-linked PEG. In doing so, the substrate is placed in a Teflon mold and then the mold is filled carefully with a solution consisting of $50 \%$ water, $50 \%$ of PEG-diacrylate $\left(M_{n}=700 \mathrm{~g} / \mathrm{mol}\right)$ and 2-Hydroxy-4'-(2hydroxyethoxy)-2-methylpropiophenone (HHMPP $1 \mathrm{mg} / \mathrm{mL}$, photoinitiator). It's worth mentioning that the side of $\mathrm{CaF}_{2}$ is marked and only the upside is used though the whole experiment. The polymerization proceeds for 3 min under UV radiation. The whole mold is put into water so the $\mathrm{CaF}_{2}$ substrate can detached from the hydrogel matrix spontaneously. It can be clearly seen that the reddish material transferred from the $\mathrm{CaF}_{2}$ surface onto the hydrogel surface (Figure 5.18). 
To validate the transfer of both AuNPs and $\mathrm{SiO}_{2}-\mathrm{NPs}$, the $\mathrm{CaF}_{2}$ substrate is analyzed with AFM. Figure 5.19 shows the micrograph of $\mathrm{CaF}_{2}$ after transfer. This figure shows clearly that both $\mathrm{AuNPs}$ and $\mathrm{SiO}_{2}$-NPs are completely removed from the surface, indicating a complete transfer of both NPs onto hydrogel. It should be mentioned here that, the interaction between NPs and the substrate is rather strong; even using mechanical stretching, both AuNPs and $\mathrm{SiO}_{2}$ cannot be efficiently removed from $\mathrm{CaF}_{2}$. This strongly suggests the nanoparticles are transferred onto the hydrogel rather than detached from the surface during the incubation in $\mathrm{CHCl}_{3}$.

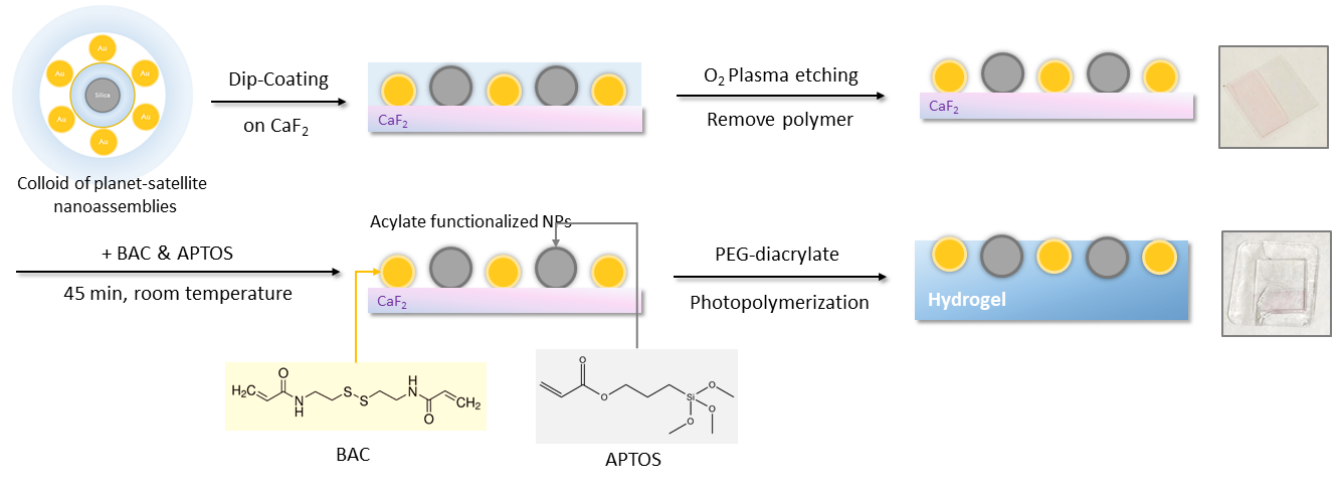

Figure 5.18: Schematic illustrated experimental procedure of transferring planetsatellite nanostructures onto hydrogel including photos for $\mathrm{CaF}_{2}$ and hydrogel samples. The reddish part of the sample is the area decorated with nanoparticles (SPR band of AuNPs). 


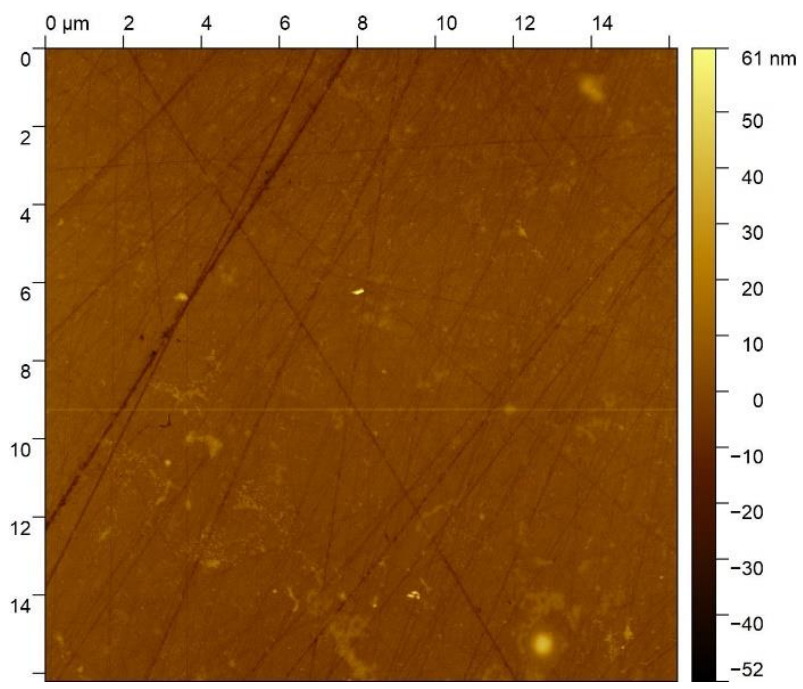

Figure 5.19: Surface of $\mathrm{CaF}_{2}$ crystal after the transfer of nanoparticles onto hydrogel. No particle can be observed on the surface.

\subsection{Conclusion}

In this section, the synthesis of silica-planet-gold-satellite nanostructures are studied in detail including fabricating of dense poly(NiPAAM) capped $\mathrm{SiO}_{2}$-NPs (the "planet") in a completely separated manner and the successive self-assembly of the planet $\mathrm{SiO}_{2}$ with satellite AuNPs.

The poly(NiPAAM) capped $\mathrm{SiO}_{2}$-NPs are fabricated through a graftingfrom approach from RAFT-capped $\mathrm{SiO}_{2}$ which is obtained via a two-step reaction. The polymerization condition is optimized in detail including the choosing of solvent and pretreatment of polymerization's colloid to archive the complete dispersion of $\mathrm{SiO}_{2}$-NPs during the polymerization. Under the optimized conditions, planet NPs can be produced without significant presence of dimer silica cores.

The conditions of the self-assembly process of the planet-satellite nanostructure are optimized to proceed at room temperature in several minutes with very high reproducibility. Owing to the flexible synthetic 
strategy, the geometry of planet-satellite nanostructure including interparticle distance, size of both planet / satellite nanoparticles, and the number of the satellite nanoparticles can be precisely tuned. From the statistic evaluation on the TEMicrograph, the interparticle distance between planet and satellite nanoparticles can be tuned from 10 to $50 \mathrm{~nm}$ by changing the chain length of the linker polymer grafted on $\mathrm{SiO}_{2}$-NPs.

From the nano-sized planet-satellite structure to the macroscopic nanopatterned surface, the dip-coating technique is applied here to create a monolayer on the $\mathrm{CaF}_{2}$ surface. We have seen in section 5.4.3 that the $\mathrm{CaF}_{2}$ substrate is not suitable for cell experiments due to insufficient passivation (PEGylation) for RGD peptide. (Although the passivation is sufficient for melanoma cells) To address this issue, a strategy for covalently transferring both AuNPs and $\mathrm{SiO}_{2}$-NPs onto hydrogel is established. 


\title{
Chapter 6
}

\section{Further Development of RAFT-Polymer Functionalized}

\author{
Nanoparticle Assemblies
}

\begin{abstract}
Owing to the inherent flexibility in designing the polymer structure and fabricating nano components, the RAFT-polymer functionalized nanoassemblies can quickly expend their utility by integrating new properties from functional polymers and further additional nanocomponents. This chapter demonstrates two distinct ubiquitous methodologies to build new features into the nanoassemblies.
\end{abstract}

The first strategy [139] focuses on the functional polymer. Sofar, poly(NiPAAM) and poly(MMA) was used for fabricating nanostructure. Section 6.1 demonstrates an efficient synthesis route for the gold/silvercore-PE-shell nanohybrids in a simple self-assembly approach using and trithiocarbonate terminated PE. Those nanoassemblies showed similar solubility as PE. DLS showcases the reversible thermoresponsive aggregation/disaggregation properties of these nanohybrids.

The second approach utilized the micro-emulsion technique used in the synthesis of the $\mathrm{SiO}_{2}$-NPs. This technique not only provides the synthesis of highly spherical $\mathrm{SiO}_{2}$-NPs, but it also gives access to a one-to-one silica coating of small $(<15 \mathrm{~nm})$ nanoparticles. Here, the magnetite nanoparticles (MNP) are introduced into the $\mathrm{SiO}_{2}$-NPs as a functional core for its superparamagnetism. The MNP is thus completely separated from each other owing to the one-to-one silica coating and polymer shells. Hence, the magnetic interaction between each MNP is effectively inhibited which is highly favored in the realm of magnetic hyperthermia applications. 


\subsection{Polyethylene-grafted gold and silver nanoparticles using catalyzed chain growth}

Polyethylene is one of the most widely used polymers for its unique properties (e.g., strong resistance to solvent, mechanical and thermal resistance) and low cost. Various composite materials for PE and nanoparticles have been synthesized by in situ polymerization yielding polymer-nanoparticle blend. However, the surface modification on the nanoparticles using PE has only a few examples of silica[140] and iron oxide[141]. To the best of author's knowledge, the surface modification with PE brush on AuNPs and AgNPs has not been reported. The major challenge here is to synthesis PE with a well-defined anchoring group for AuNPs and AgNPs.

Here, catalyzed chain growth technique is applied to obtain magnesium end-functionalized $\mathrm{PE}$. Using an $\mathrm{Nd}$ based metallocene precatalyst $\left(\left(\mathrm{cp}^{*}\right)_{2} \mathrm{NdCl}_{2} \mathrm{Li}(\mathrm{OEt})_{2}\right)$ in a combination of a diorganomagnesium compound is used as an activator and chain transfer agent. (Figure 6.1). By successive reaction with bis(benzylsulfinyl thiocarbonyl)disulfide, the RAFT group can be introduced to the PE as end-termini.[142] An additional reaction of RAFT terminated PE with hydrazine can yield thiol terminated PE. The molar mass of the yielded PE is determined by GPC $\left(M_{\mathrm{p}}=2.5 \cdot 10^{3} \mathrm{~g} \cdot \mathrm{mol}^{-1}\right)$

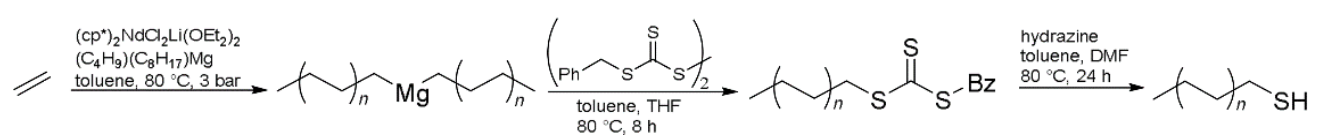

Figure 6.1: Synthesis route for trithiolcarbonate and thiol terminated PE. Adapted from ref. [139] with permission from Wagner, J; Peng, W; Vana, P. Polymers 2018, 10(4), 407. Open access.

The end-group functionalized PE is ready for the self-assembly of coreshell-structures with AuNPs and AgNPs. Due to the limited solubility of PE (only in unpolar solvent, e.g., toluene), the utilized AuNPs and AgNPs should 
possess excellent dispersibility in solvent like toluene. Base on this consideration, tetraoctylammonium bromide (TOAB) capped gold from Brust-Schiffrin synthesis and oleylamine capped silver nanoparticles are applied here.

Grafting of both RAFT and thiol terminated PE to the surface AuNPs and AgNPs can be archived sharing the same simple protocol: in a typical run, end-group functionalized PE was dissolved in toluene at $90{ }^{\circ} \mathrm{C}$ to achieve a complete solution of the polyethylene chains. Toluene colloid containing metal NPs were injected into the polymer solution and stirred for $30 \mathrm{~min}$ at $90^{\circ} \mathrm{C}$. To avoid oxidation of the silver particles at higher temperatures, the process was performed under an argon atmosphere.

Transmission electron microscopy was performed (Figure 6.2). Comparing the images of unfunctionalized $\mathrm{Au} / \mathrm{AgNPs}$ with PE capped $\mathrm{Au} / \mathrm{AgNPs}$, an increase in interparticle distance can be clearly seen for the $\mathrm{PE}$ capped $\mathrm{Au} / \mathrm{AgNPs}$ indicating the formation of the PE shell. Furthermore, there is no significant difference between RAFT terminated PE and thiol terminated PE in any analysis and experiment. 


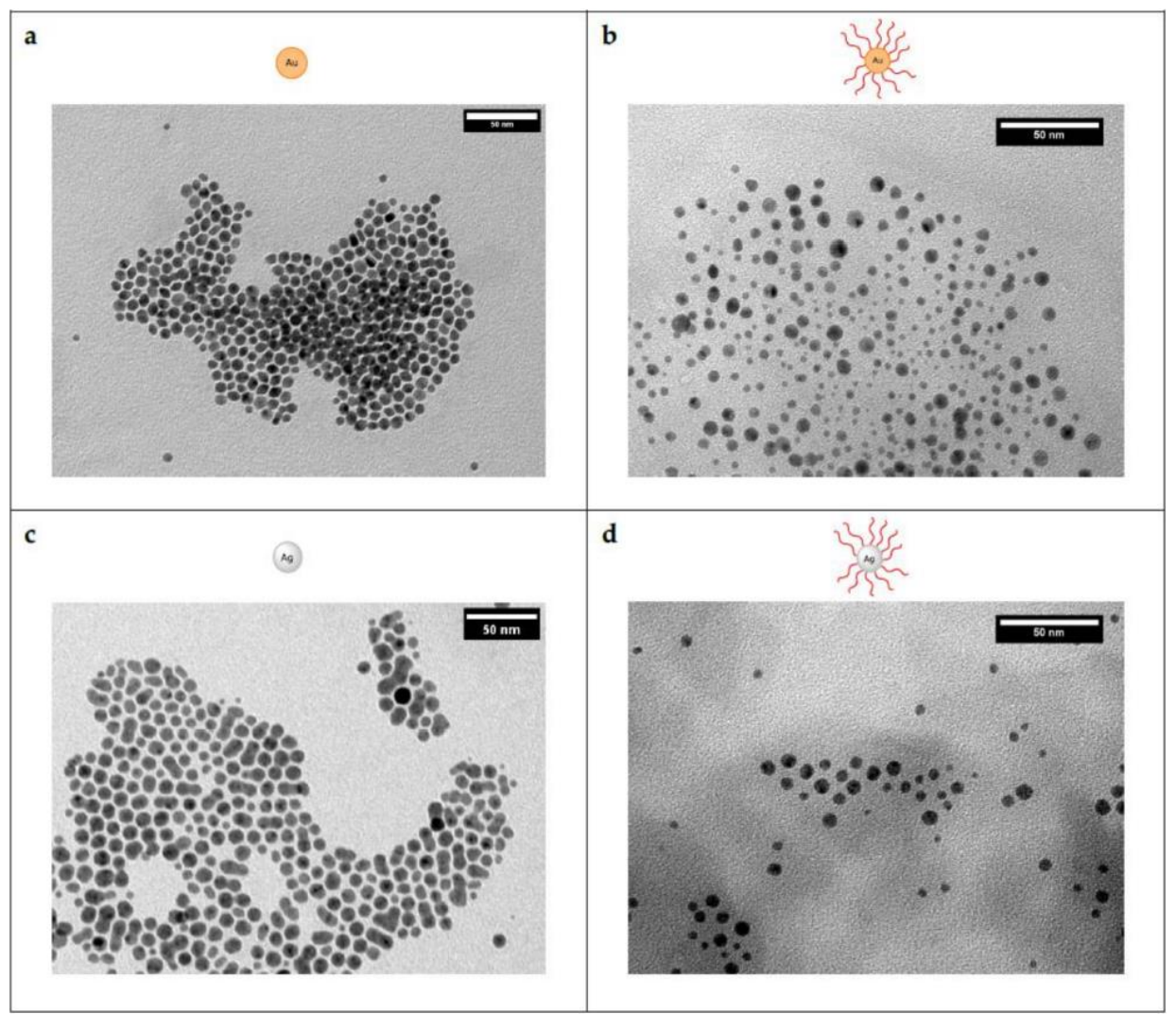

Figure 6.2: TEMicrograph of a) $\sim 5 \mathrm{~nm}$ Brust-Schiffrin AuNPs, b) PE functionalized AuNPs, c) Oleylamine capped AgNPs, d) PE capped AgNPs. Adapted from ref. [139] with permission from Wagner, J; Peng, W; Vana, P. Polymers 2018, 10(4), 407. Open access.

The PE capped AuNPs and AgNPs inherit the unique solution property of the PE. Figure 6.3 demonstrates the temperature dependence of the PE functionalized AuNPs(left) and AgNPs(right): Both nanoparticles have perfect dispersibility above the solution temperature of PE $\left(80^{\circ} \mathrm{C}\right)$ while precipitating under $80^{\circ} \mathrm{C}$. DLS reveals the increasing hydrodynamic radius of unfunctionalized NPs $(\sim 10 \mathrm{~nm})$, PE capped NPs in toluene at $90{ }^{\circ} \mathrm{C}$ $(\sim 25 \mathrm{~nm})$ and $\mathrm{PE}$ capped NPs in toluene at $25{ }^{\circ} \mathrm{C}$ (aggregated). The transition temperature is determined for PE capped AuNPs using UV-Vis spectroscopy. By monitoring the $530 \mathrm{~nm}$ peaks of AuNPs (surface plasmonic resonance absorption peak), the presence of dispersed AuNPs in the colloid can be precisely detected. The spectra were taken in $5{ }^{\circ} \mathrm{C}$ steps with $2 \mathrm{~h}$ 
equilibrium time between each measurement. Figure 6.4 demonstrates the temperature dependence of the dispersed number of AuNPs. A sharp transition can be seen in Figure 6.4(left) between $65^{\circ} \mathrm{C}$ and $75^{\circ} \mathrm{C}$. Furthermore, the PE-AuNPs has undergone several temperature cycles between $25^{\circ} \mathrm{C}$ and $90^{\circ} \mathrm{C}$ while monitoring its absorption at $530 \mathrm{~nm}$. The temperature cycles in Figure 6.4(right) demonstrates the completely reversible temperature-dependent aggregation-dispersion property of the PE capped AuNPs.

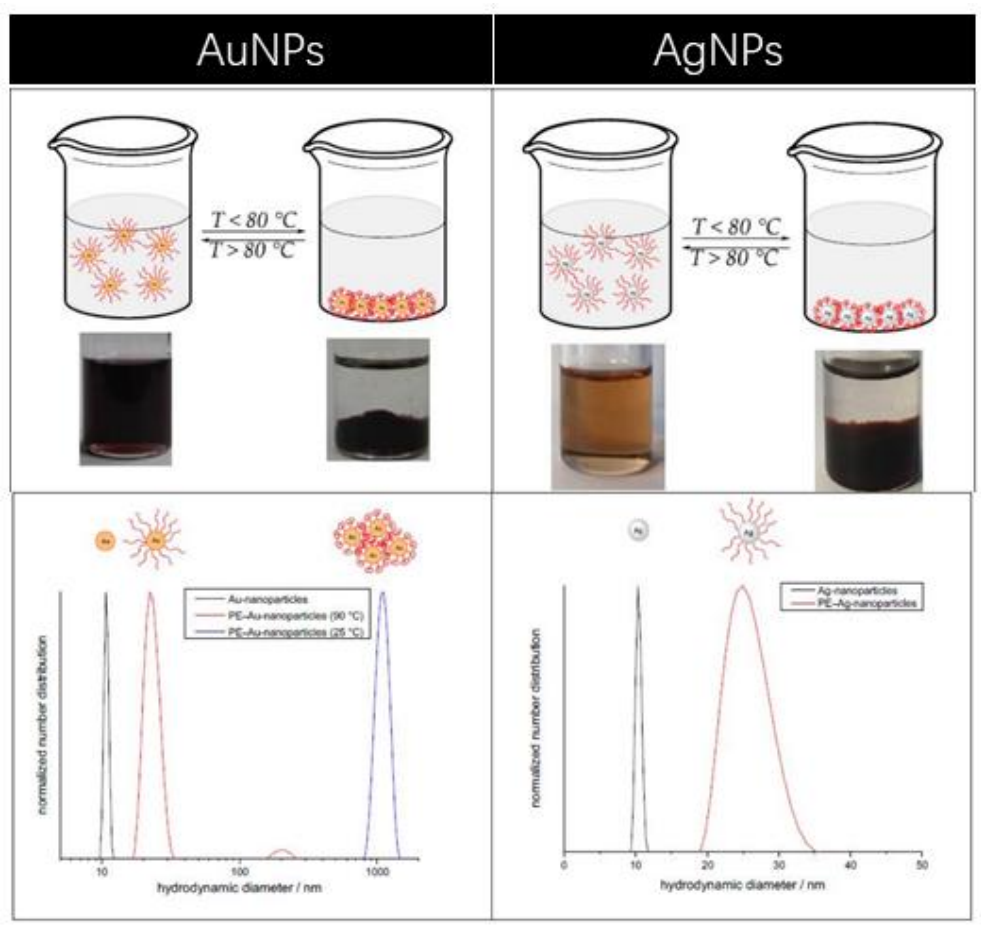

Figure 6.3: Upper: Temperature-dependent solubility of polyethylene grafted gold and silver nanoparticles. Lower: Intensity distributions from dynamic light scattering measurements of unfunctionalized and polyethylene-functionalized gold- and silvernanoparticles. The data for PE-AgNPs at room temperature is not avaliable because the size of the aggregate is too large for DLS to measure. Adapted from ref. [139] with permission from Wagner, J; Peng, W; Vana, P. Polymers 2018, 10(4), 407. Open access. 

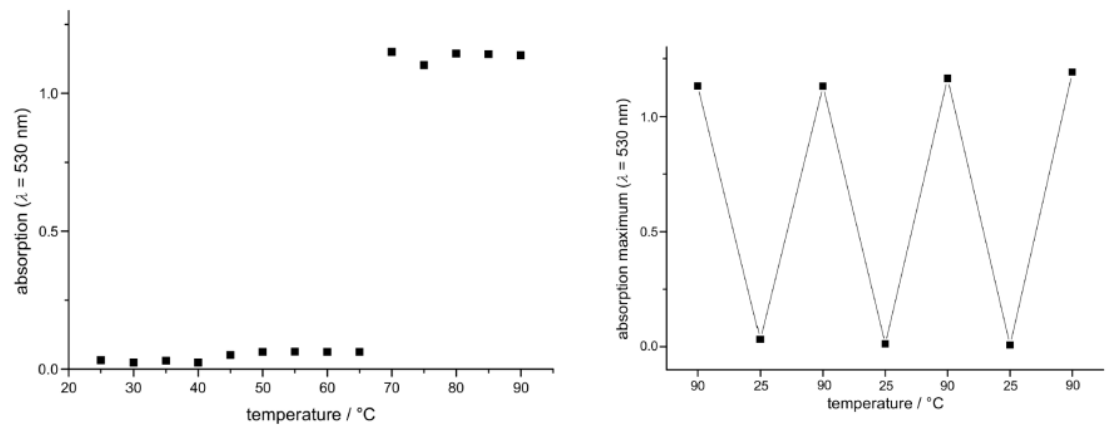

Figure 6.4: UV-vis measurement for temperature change (left) and cycling (right) on PE-AuNPs in toluene. The low value of absorption at $25 \mathrm{C}$ indicates the absence of PEAuNPs in the light pathway, which is located in the middle of the cuvette. In this case, PE-AuNPs are segregated. Adapted from ref. ${ }^{[139]}$ with permission from Wagner, J; Peng, W; Vana, P. Polymers 2018, 10(4), 407. Open access.

Beyond the temperature-dependent solution behavior, a further intriguing property of the PE capped AuNPs/AgNPs is the perfect dispersibility in the PE matrix. Figure 6.5 shows the TEMicrograph of PE matrix containing low $(\sim 4 \mathrm{w} \%)$ and high $(\sim 10-15 \mathrm{w} \%)$ NPs. It can be clearly seen that both AuNPs and AgNPs can be perfectly dispersed in the PE matrix without any degree of aggregation. 


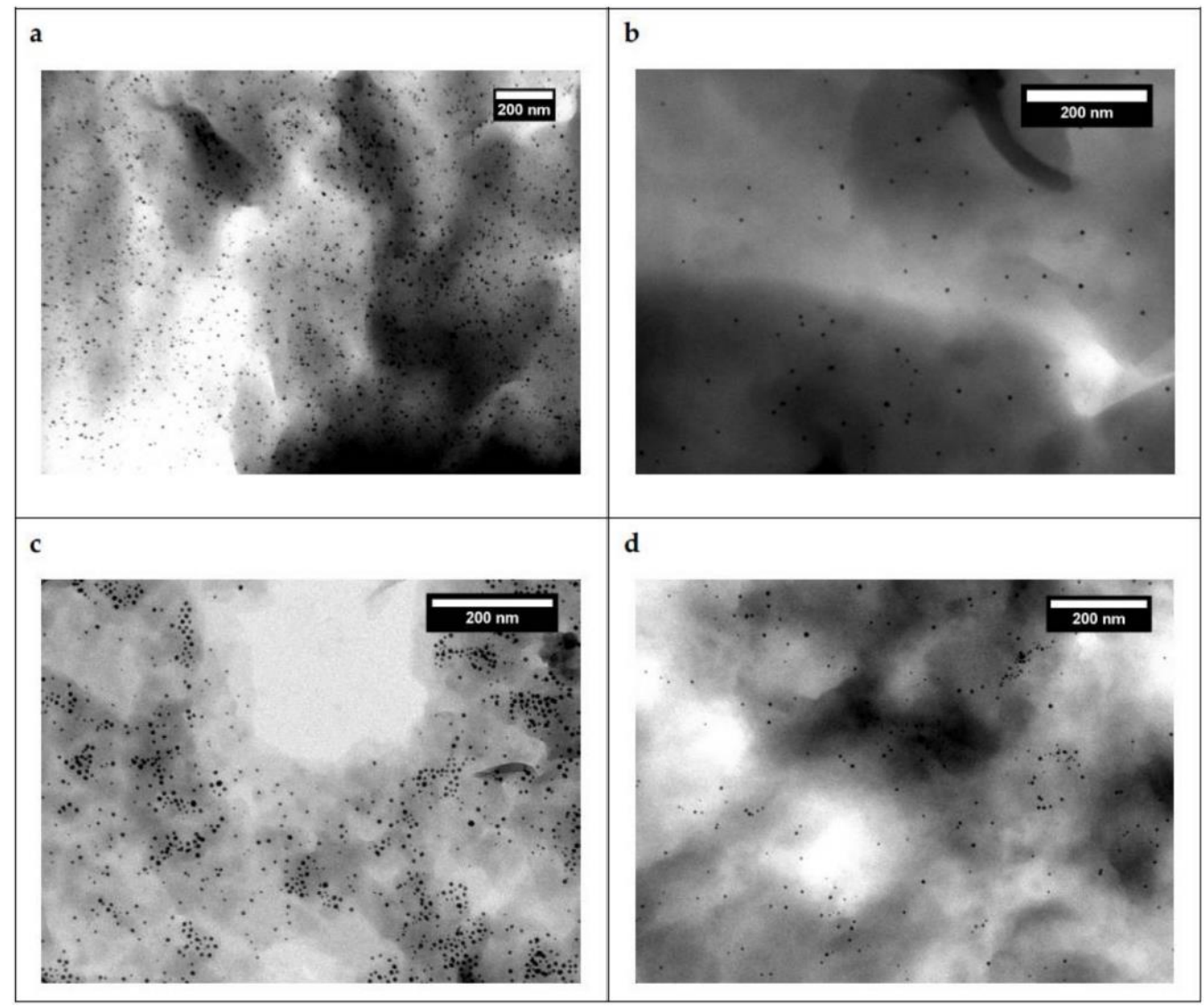

Figure 6.5: TEMicrographs of dispersed PE-AuNPs with a high (a) and low (b) particle content and for PE-AgNPs with high (c) and low (d) particle content in a polyethylene matrix. Adapted from ref. [139] with permission from Wagner, J; Peng, W; Vana, P. Polymers 2018, 10(4), 407. Open access.

In conclusion, these results validated the general feasibility of RAFT/thiol terminated polymer as a powerful tool to functionalize both AuNPs and AgNPs by applying a simple and effective protocol. The PE functionalized AuNPs and AgNPs possesses very high colloidal stability in terms of time and thermal stability. The temperature-dependent solubility was introduced to the PE functionalized NPs. The PE shell further provides a perfect dispersibility of metal nanoparticles in the PE matrix. 


\subsection{Silica-coated magnetite nanoparticles carrying a high- density polymer brush shell of hydrophilic polymer}

This section demonstrates a simple and versatile strategy for fabricating hydrophilic polymer (PNiPAAM) capped magnetite-core-silica-shell nanohybrids. The nanoassemblies possess a well-defined structure integrating the favorable superparamagnetic property of MNP with the functional polymer. The dense polymer shell enables a precise patterning of the magnetic nanohybrids with a tunable interparticle distance ranging from $20 \mathrm{~nm}$ to $80 \mathrm{~nm}$. The silica and polymer also play a very effective role at shielding the magnetic interaction between MNPs which is highly on demand for magnetic hyperthermia applications. SQUID measurement proves the effectively inhibited magnetic interaction.

Magnetite nanoparticles have attracted huge research interest over recent years for extensive applications especially in medical field, e.g., magnetic resonance imaging,[143-148] drug-delivery, [149-151] tumor treatment (hyperthermia).[152-155] In the field of in vivo studies, dispersibility, arrangement, and interaction between MNPs often decide the performance of MNP.[154,156] Especially for the magnetic hyperthermia, the aggregation can cause huge performance diminishing due to the strong interaction between MNPs in the aggregated state.[154,156] On this point, surface modification of MNPs is inevitably important to control and optimize the interparticle interaction. The basic idea in this work to monitor the interaction between MNPs is by using $\mathrm{SiO}_{2}$ and polymer brush as a spacer to separate MNPs: one-to-one silica coating is the key step here to isolate MNPs from each other permanently. Furthermore, intensively studied functional

polymer brush on nanoparticles provides many advantages, e.g., enhanced biocompatibility and colloidal stability.[53]

$\sim 8 \mathrm{~nm}$ oleylamine-capped MNPs from a thermal decomposition synthesis ${ }^{[157]}$ is chosen here as a superparamagnetic core. To achieve the 
one-to-one silica coating on the MNPs, the reverse microemulsion method ${ }^{[64,158,159]}$ is applied here. This method also enables fine control of the shell thickness on the nanoscale (13.9 $\pm 2.1 \mathrm{~nm}$ in this work). The desired

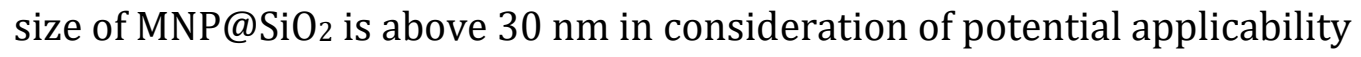
for intravenous medical applications [151]. TEMicrograph (Figure 6.6) shows

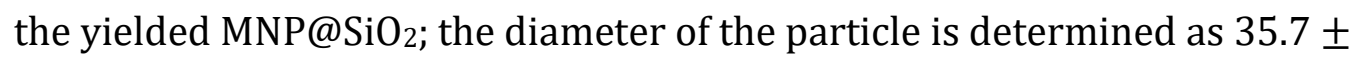
$3.7 \mathrm{~nm}$ which meets well the size requirement.

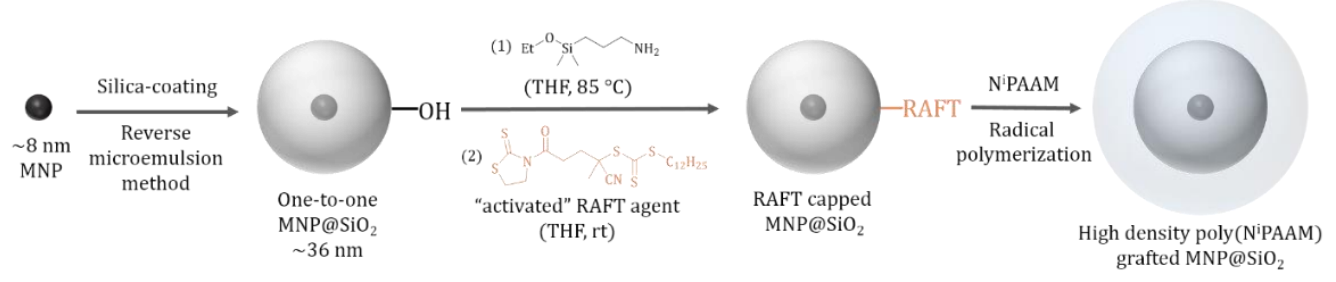

Figure 6.6: Synthetic scheme for the preparation of high-density poly(NiPAAM)-grafted $\mathrm{MNP@SiO_{2 }}$ nanohybrids. Adapted with permission from ref. [65]. Copyright 2018 John Wiley \& Sons, Inc.

The MNP@SiO 2 is then ready for the surface-initiated RAFT polymerization with NiPAAM. The experimental condition for immobilizing RAFT agent onto the surface of $\mathrm{SiO}_{2}$-NPs and the successive surface-initiated polymerization is the same as described in section 5.2. The whole synthetic scheme is summarized in Figure 6.6. Using this method, dense poly(NiPAAM) capped Figure 6.7 demonstrates the highly ordered hexagonal pattern of $\mathrm{MNP} @ \mathrm{SiO}_{2}$ owing to the narrow size distribution of the grafted polymer. The relationship between interparticle distance and molecular weight of the grafted polymer can be well reproduced here comparting with the result in Figure 6.7 of the poly(NiPAAM) grafted $\mathrm{SiO}_{2}$ NPs under the analogous experimental condition. (Polymerization's condition is summarized in Table 7.2 in Section 7.4)

Now we focus on the magnetic properties of the nanoassemblies. Magnetic characterization of the resulting core-shell particles was performed using a superconducting quantum interference device (SQUID, 
Figure 6.9). Owing to the well-defined structures, a coherent study of the influence of silica coating and polymer brush on the magnetic properties and the interparticle magnetic interaction can be accomplished. Figure 6.9a shows no observable hysteresis loop indicating the superparamagnetic behavior of all the samples at room temperature. The specific saturation magnetization $\left(M_{\mathrm{s}}^{\text {sample }}\right)$ decreases significantly when going from pure nanoparticles to silica-coated and finally to polymer-brush coated particles. However, if we normalize $M_{\mathrm{s}}$ to the mass of the MNPs cores, $M_{\mathrm{s}}^{\text {core }}$, the value

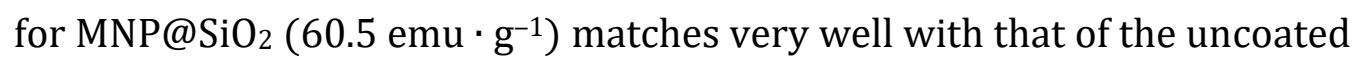
MNPs $\left(61.0 \mathrm{emu} \cdot \mathrm{g}^{-1}\right)$, showing that the decrease in the "apparent $M_{\mathrm{s}}$ " simply originates from the decreasing mass ratio of magnetite when covering with silica and grafted polymer. The ZFC/FC curves give insight into the interaction between MNPs in different samples. The significant broadening of the ZFC peak in Figure 6.9b for the unfunctionalized MNP is not mainly caused by inhomogeneous particle size distribution, but by magnetic interactions between the particles ${ }^{[160]}$. Once the MNPs are coated with silica or further capped with polymer brush, the ZFC peak becomes narrower and the FC curve at low temperature $(<50 \mathrm{~K})$ becomes convex. Both changes indicate a reduced interaction between MNPs as a consequence of their greater distance. At blocking temperature $\left(T_{\mathrm{B}}\right)$, the transition between superparamagnetism and blocked state occurs. In general, $T_{\mathrm{B}}$ decreases with increasing interparticle distances of the MNPs ${ }^{[161]}$. TB decreases significantly from $72 \mathrm{~K}$ of unfunctionalized MNPs to $33 \mathrm{~K}$ for both coated MNPs. These results consist well with the announcements from a computational study ${ }^{[162]}$. The magnetic dipole interaction can be neglected if the MNPs are fixed in the matrix and separated from each other by a distance larger than three times of their diameter ( $d=7.9 \mathrm{~nm}$, distance between MNPs $>30 \mathrm{~nm}$, in our case). The above results impressively demonstrate that the magnetic dipole interaction of the MNP cores is very effectively reduced to a negligible level. 


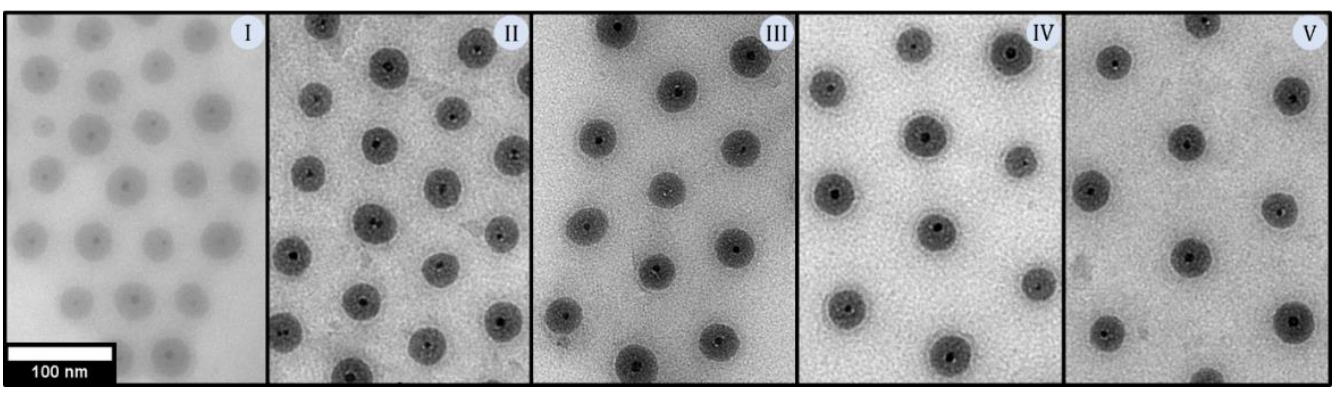

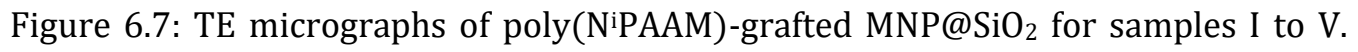
$\mathrm{MNP@SiO} 2$ are found in hexagonal patterns with distinct spacing. TEM images with lower magnification are shown in supporting information. Adapted with permission from ref. [65]. Copyright 2018 John Wiley \& Sons, Inc.

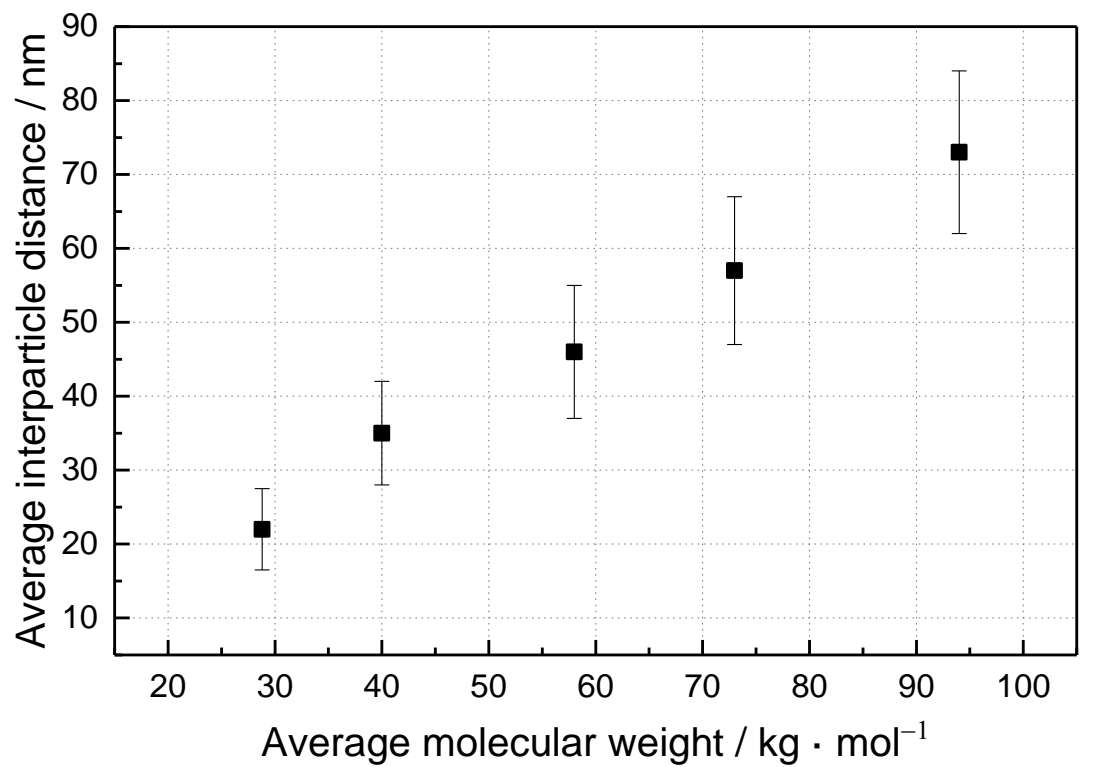

Figure 6.8: Average interparticle spacing independence of poly(NiPAAM)-grafted MNP@SiO ${ }_{2}$ samples I to $\mathrm{V} v s . M_{\mathrm{n}}$ of the free polymers. Adapted with permission from ref. [65]. Copyright 2018 John Wiley \& Sons, Inc. 
(a)

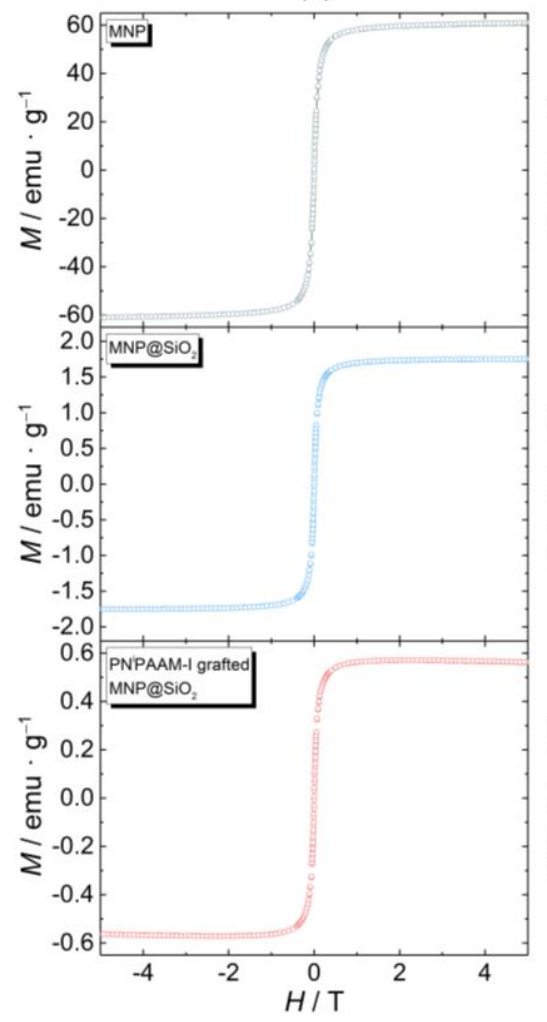

(b)

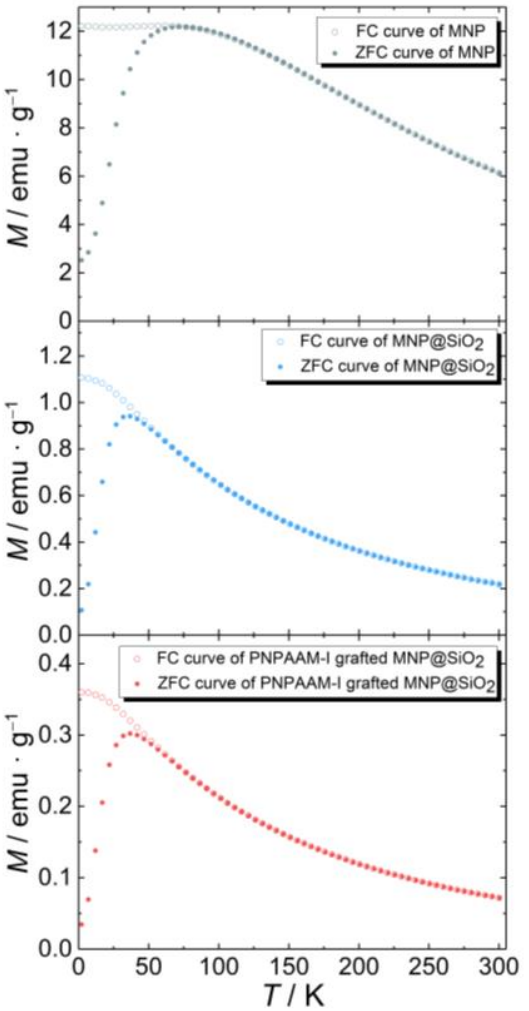

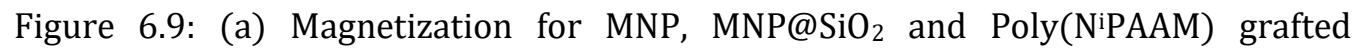

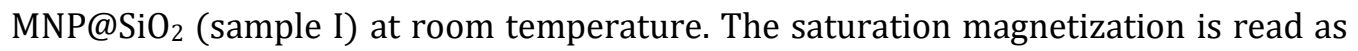
61.0, 1.75 and $0.56 \mathrm{emu} \cdot \mathrm{g}^{-1}$ for mass normalization of the sample. (b) Zero field cooled/field cooled (ZFC/FC) measurements of the corresponding sample at $H=100$ Oe. Adapted with permission from ref. [65]. Copyright 2018 John Wiley \& Sons, Inc.

\subsection{Conclusion}

This chapter demonstrates two different pathways to introduce further functionality to the RAFT polymer functionalized nanoparticles. In the first approach, polyethylene brush is successfully introduced to both AuNPs and AgNPs by using RAFT or thiol terminated PE. The PE functionalized AuNPs and AgNPs possess the temperature-dependent solubility of PE. DLS and UV- 
Vis spectroscopy were applied to monitor this transition. The transition's temperature is determined between 65 and $70^{\circ} \mathrm{C}$ and this transition is completely reversible over many cycles. Furthermore, these PE capped metal nanoparticles hybrid and can be perfectly dispersed in PE matrix with very high NPs content and long-term stability.

The second approach is to introduce an extra functional nanocore into the silica. $\sim 8 \mathrm{~nm}$ Magnetite NPs is chosen as a superparamagnetic core. Using the $\mathrm{w} / \mathrm{o}$ reverse microemulsion technique, the one-to-one silica coating of MNP can be archived yielding $\sim 36 \mathrm{~nm} \mathrm{SiO}{ }_{2}$-NPs carrying a single MNP core. The RAFT group is then anchored onto the surface with successive surface-initiated polymerization as described in section 5.2. Polymer capped MNP@SiO2 can self-assmebly into a 2D hexagonal pattern using simply drop-casting. The interparticle distance can also be precisely tuned by the chain length of the grafted polymer. SQUID measurements validate the effectively inhibited magnetic interaction between MNP cores due to the silica coating and polymer shell. This character is particularly favorable for the hyperthermia applications. 


\title{
Chapter 7
}

\author{
Experimental
}

\subsection{Chemicals}

Silica capped magnetite nanoparticles with magnetite core are kindly provided by Yingying Cai from the institute of physical chemistry at the University of Göttingen. Thiol and RAFT terminated polyethylene is kindly provided by Jannik Wagner from the institute of physical chemistry at the University of Göttingen. The 4-arm star RAFT agent is kindly provided by Christian Roßner from the institute of physical chemistry at the University of Göttingen. PLL(20 kDa)-g-PEG(20 kDa) solution is kindly provided by Katharina-Amschler from the university medical center Göttingen. Ethanol (Sigma-Aldrich, 99.8\%), oleylamine (ACROS, approximate C18-content 8090\%), 3-aminopropyldimethylethoxysilane (abcr, 97\%), IGEPAL ${ }^{\circledR}$ CO-520 (Average $M_{\mathrm{n}}=441 \mathrm{~g} \cdot \mathrm{mol}^{-1}$, Sigma-Aldrich), $n$-hexane (VWR, 98.4\%), dimethylacetamide (Fluka ,HPLC), chloroform (Fischer, HPLC), cyclohexane (VWR, $\geqslant 99 \%$ ), aqueous ammonia (VWR, $32 \%$ ), tetraethyl orthosilicate (Sigma Aldrich, $\geqslant 99 \%$ ), mercaptothiazoline (Sigma Aldrich, 98\%), 1,4dioxane (ROTH, $\geqslant 99.5 \%$ ), dicyclohexylcarbodiimide (Sigma-Aldrich, 99\%), 4-(dimethylamino)pyridine (Sigma-Aldrich, 99\%), 4-cyano-4(dodecylsulfanyl-thio-carbonyl)-sulfanylpentanoic acid (CDSPA, abcr, $\geqslant$ 97\%), DMF (Sigma-Aldrich, 99.8\%), methanol (Sigma-Aldrich, 99.8\%), sodium citrate tribasic dihydrate (Sigma-Aldrich, 99\%), diisopropylether (ACROS, 99\%), toluene (Fischer, HPLC), $\mathrm{AgNO}_{3}$ (Fluka, 98\%), hydrogen tetrachloroaurate trihydrate (ABCR, 99.9\%), cyclic RGD peptide (Cyclo(RGDfE)-PEG-5-CCC, Peptide Specialty Laboratories, Heidelberg), PEG-diacylate $\quad\left(M_{\mathrm{n}}=480 \mathrm{Da}, \quad\right.$ Sigma-Aldrich $), \quad$ 2-hydroxy-4'-(2- 
hydroxyethoxy)-2-methylpropiophenome (TCI, >98\%), N,N'-bis(acryloyl)cystamine (Alfa Aesar, 98\%), 3-acryloxypropyltrimethoxysilane (Sigma-Aldrich, 92\%), $\mathrm{CaF}_{2}$ crystal (Korth Kristalle) were used as received.

$\mathrm{N}$ PAAM was recrystallized twice from toluene/hexane $(3: 1)$ and stored at $3{ }^{\circ} \mathrm{C}$ prior to use. AIBN (Fluka, 98\%) was recrystallized twice from diisopropylether and stored at $-20^{\circ} \mathrm{C}$ prior to use. Tetrahydrofuran (Acros, 99.9\%) was dried over $\mathrm{CaH}_{2}$ overnight and distilled before use. Nanopure (type I) water was obtained from a Millipore water purification system equipped with a UV lamp (electric resistivity $18.2 \mathrm{M} \Omega \cdot \mathrm{cm}$ ).

\subsection{Equipment and analytical methods}

\section{Size-exclusion chromatography (SEC)}

SEC characterization of poly(NiPAAM) was performed at $45{ }^{\circ} \mathrm{C}$ with DMAc containing $0.1 \%$ LiBr as eluent on an Agilent 1260 Infinity SEC system with solvent pump, a PSS GRAM precolumn ( $8 \times 50 \mathrm{~mm} ; 5 \mu \mathrm{m}$ particle size $)$, three PSS GRAM separation columns (PSS SDV; $8 \times 300 \mathrm{~mm} ; 30 \AA ., 10^{3} \AA$ and $10^{3} \AA$ pore sizes). An Agilent RI detector and an Agilent UV detector were applied. The flow rate was set at $0.8 \mathrm{~mL} \cdot \mathrm{min}^{-1}$. The setup was calibrated with linear poly(methylmethacrylate) standards with low dispersity. All samples $\left(5 \mathrm{~g} \cdot \mathrm{L}^{-1}\right)$ were filtered through a $0.45 \mu \mathrm{m}$ PTFE filters prior to injection.

For star polymers measured on this SEC set up the results for average molar masses were multiplied with a factor of 1.3. [131]

\section{Transmission electron microscopy (TEM)}

TEM measurements were conducted on a Philips CM 12 TEM. The TEM was operated at an acceleration voltage of $120 \mathrm{kV}$ and an emission current of 3-4 $\mu \mathrm{A}$. The focused electron spot has a diameter of $10 \mu \mathrm{m}$. A $50 \mu \mathrm{m}$ aperture was used in the condenser lens and all scattered electrons were 
blocked with a $20 \mu \mathrm{m}$ aperture. The instrument was equipped with an Olympus $1376 \times 1032$ pixel CCD-camera. All samples were prepared on Plano 200 mesh copper TEM grid holding an amorphous carbon film by the drop-casting method and slowly evaporation of the solvent. The TEM grids were placed on a laboratory paper tissue, and one drop of a mixture was given onto them through a glass pipette. The TEM grid was immediately covered with a glass cup until all solvent had evaporated.

\section{High-resolution transmission electron Microscopy (HRTEM) and high angle annular dark-field scanning transmission electron microscope (HAADF-STEM)}

HRTEM and HAADF-STEM images were acquired on a FEI Titan 80-300 Environmental TEM equipped with a corrector of spherical aberration at the image side and a Gatan Imaging Filter (GIF) Quantum 965ER. The microscope was operated at $300 \mathrm{kV}$. The inner and outer electron collecting angle of the HAADF detector was set to be 70 and 200 mrad respectively, and the probe semi-convergence angle was about $10 \mathrm{mrad}$. The spectrum images were composed using $\mathrm{L}_{2,3}$-edge of silver and $\mathrm{M}_{4,5}$-edge of gold.

\section{UV-Vis spectroscopy}

Optical absorption spectroscopy was performed with a Cary 300 scan photo spectrometer in solution against pure solvent (baseline subtraction method). Hellma quartz cuvettes with a thickness of $10 \mathrm{~mm}$ were used as sample holder. The scan rate was $150 \mathrm{~nm} \cdot \mathrm{min}^{-1}$ in steps of $0.5 \mathrm{~nm}$.

\section{Dynamic light scattering (DLS)}

The DLS was performed on a Malvern Zetasizer Nano S system operating at $633 \mathrm{~nm}$ at scattering angle of $173^{\circ}$. Dilute samples were passed through $0.45 \mu \mathrm{m}$ PTFE filters. The measurements were conducted at $25^{\circ} \mathrm{C}$ if not specially mentioned. 


\section{Thermogravimetric analysis (TGA)}

Thermogravimetric analysis (TGA) was carried out using a TG 209 F3 Tarsus analyzer in the temperature range from 25 to $1000{ }^{\circ} \mathrm{C}$ at a heating rate of $10^{\circ} \mathrm{C} / \mathrm{min}$. The experiments were performed under $\mathrm{N}_{2}$ gas at a rate of $20 \mathrm{~mL} / \mathrm{min}$.

\section{Super quantum interference device (SQUID) measurement}

SQUID measurement was carried out on a Quantum Design MPMS-XL-5 magnetometer. ZFC/FC curves were recorded with a static field of $100 \mathrm{Oe}$ in the range from 295 to $2 \mathrm{~K}$.

\section{Atomic force microscopy (AFM)}

Atomic force microscopy measurement was carried out on a Bruker Multimode 8 equipped with Scanasyst-Air with the resonance frequency of $70 \mathrm{kHz}$, spring constant of $0.40 \mathrm{~N} / \mathrm{m}$. The measurement was conducted via NanoScope software.

\section{Plasma cleaner}

The plasma etching was conducted using a Diener Zepto plasma cleaner. The plasma cleaner operated at 0.4 mbar air using a $40 \mathrm{kHz} 100 \mathrm{~W}$ generator.

\section{UV curing chamber}

The photopolymerization of PEG-diacrylate was carried out in a curing chamber Hönle UVACUBE inert equipped with flooded $\mathrm{CO}_{2}$. A standard mercury lamp with a power of $100 \mathrm{~W} / \mathrm{cm}$ was used here.

\section{Bath sonicator}

An Elmasonic S30H bath-type ultrasonicator was applied for dispersing nanoparticles from the centrifugation process or as pre-treatment for polymerization. The sonicator operated with an ultrasonic frequency of $37 \mathrm{kHz}$ and an effective sonication power of $80 \mathrm{~W}$. The operation 
temperature of the water was kept below $50{ }^{\circ} \mathrm{C}$. The sample waa placed in the center of the bath using a metal clamp.

\section{Tip sonicator}

A Fischerbrand Model 120 sonic dismembrator was applied to disperse $\mathrm{SiO}_{2}$-NPs in the centrifugation/redispersion cycles. The tip-sonicator operates at $20 \mathrm{kHz}$ with a max power of $120 \mathrm{~W}$. The actual used power must be adjusted to suit the size of the samples.

\subsection{Synthesis of nanoparticles}

\section{Synthesis of citrate-capped gold nanoparticles}

The wet-chemical synthetic procedure has been described in literature. ${ }^{[17]}$ All glassware was cleaned by aqua regia and rinsed many times with nanopure water and oven-dried prior to the experiments.

In a typical synthesis, $5 \mathrm{~mL}$ hot solution of sodium citrate $(5 \mathrm{~mL}, 39 \mathrm{mM}$, 3.9 equiv.) was added to a boiling solution of $\mathrm{HAuCl}_{4}(100 \mathrm{~mL}, 0.5 \mathrm{mM}, 1.0$ equiv.) under vigorous stirring. The mixture was refluxed for further 15 minutes and cooled slowly to room temperature. The colloidal suspension was stored in polypropylene tubes in the dark at room temperature.

To prepare the TEM sample, a small amount $(3 \mathrm{ml})$ of as-prepared AuNP sol is transferred into chloroform containing octadecylamine. The synthesis typically yielded AuNPs with a diameter of $12.7 \pm 0.8 \mathrm{~nm}$ (Figure 7.1). 

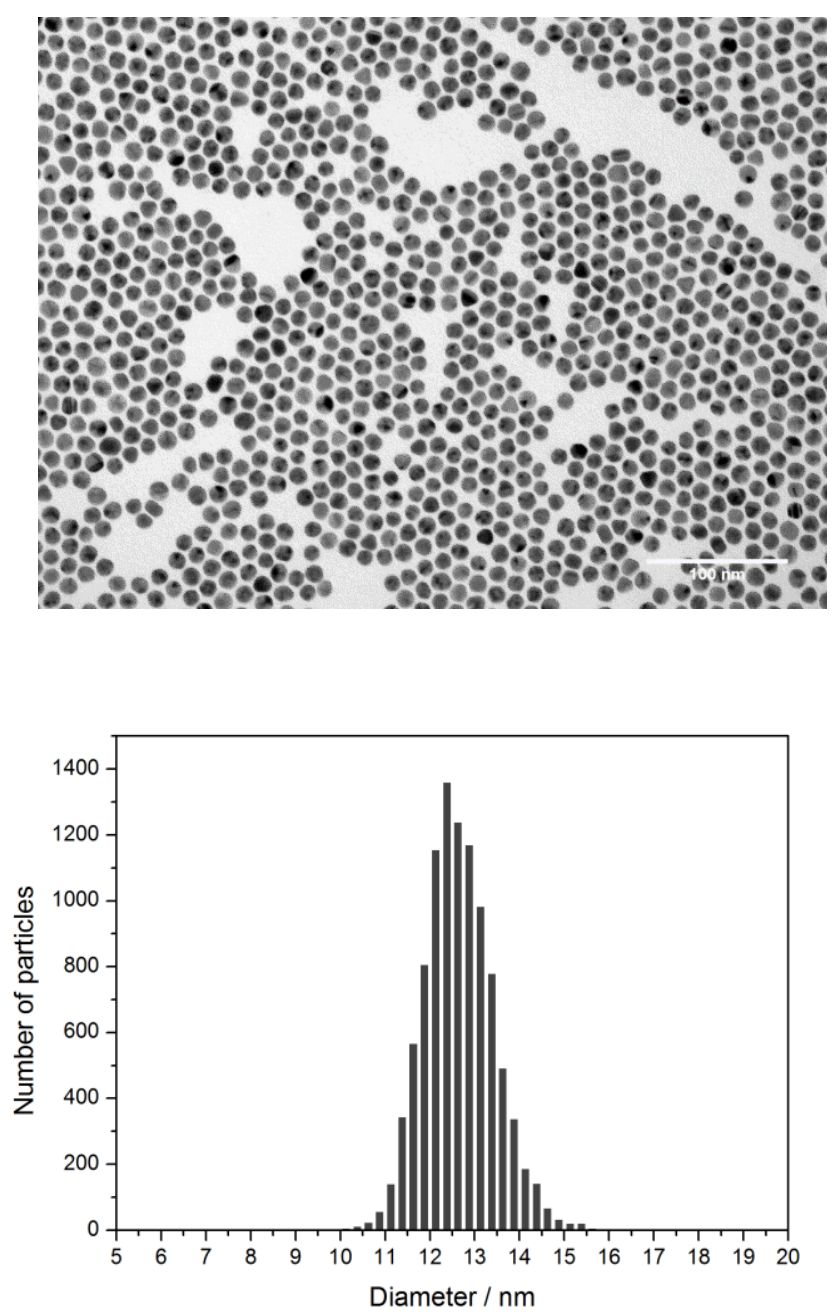

Figure 7.1: Exemplary TEM image and size distribution histogram of citrate-capped AuNPs. Adapted with permission from ref. [74]. Copyright 2016 American Chemical Society.

Synthesis of oleylamine capped silver nanoparticles

$\sim 9 \mathrm{~nm}$ silver nanoparticles were synthesized following a method reported by Hiramatsu et al.[132] In a typical experiment, $51 \mathrm{mg} \mathrm{\textrm {AgNO } _ { 3 }}$ $(0.30 \mathrm{mmol}, 1$ equv. $)$ was dissolved in oleylamine $(2.00 \mathrm{~g}, 7.48 \mathrm{mmol}$, 24.9 equiv.) under sonication. The oleylamine solution was then injected to 
$50 \mathrm{ml}$ refluxing toluene, which was purged with argon for 15 minutes. The solution was left stirring for $16 \mathrm{~h}$ under argon in order to prevent oxidation of the AgNPs. The color of the solution turned slowly from slightly yellow to orange then to dark yellow. After the reaction, the mixture was cooled to room temperature and concentrated to about $15 \mathrm{~mL}$ via rotary evaporation. $160 \mathrm{~mL}$ of methanol was added to the mixture to yield a yellowish suspension. The colloidal suspension was centrifuged at $956 \mathrm{G}$ for $20 \mathrm{~min}$ and the supernatant was removed. The segregated material was redispersed in $12 \mathrm{~mL}$ hexane and precipitated again with $80 \mathrm{~mL}$ of methanol. The segregated material could be gathered through centrifugation (27 G, 5 min) and finally redispersed in chloroform for further experiments. AgNPs which had been subjected to two centrifugations were named "AgNP washed $2 x$ ".

An additional redispersion-centrifugation cycle (hexane/methanol) could be applied in order to gain further removal of the OA on AgNPs. After the additional centrifugation process, the segregated material is redispersed in $12 \mathrm{~mL}$ chloroform for further experiments. AgNPs which had been subjected to three centrifugations were named "AgNP washed $3 x^{\prime}$. The assynthesized AgNPs were applied for further experiments immediately after the work-up process.

This synthesis typically yielded OA-capped AgNPs with a diameter of $9.2 \pm 2.6 \mathrm{~nm}$. Earlier synthesis of AgNPs has been conducted under ambient air. The resulted AgNPs can be dispersed in methanol, showing an insufficient capping of OA on AgNPs caused by oxidation at the high reaction temperature. 

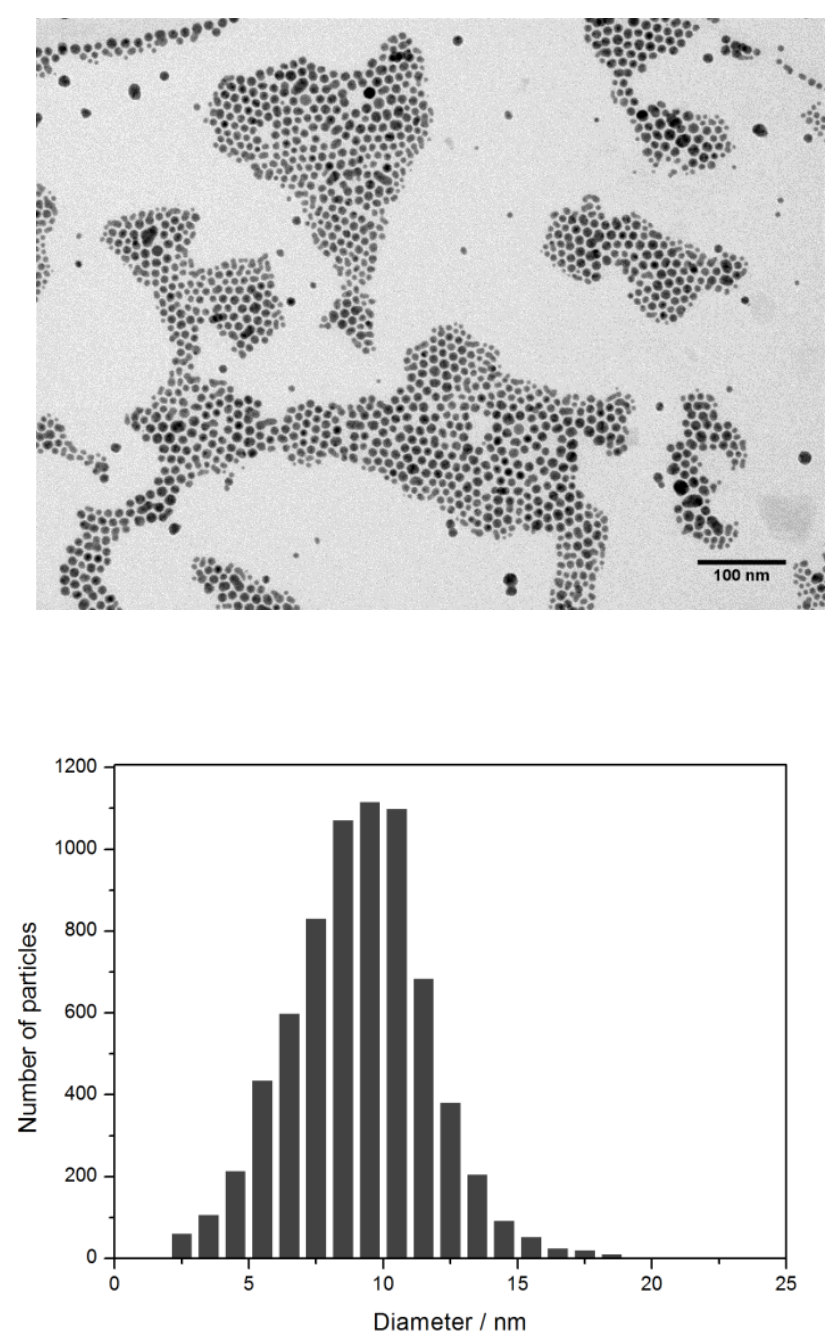

Figure 7.2: Exemplary TEM images and size distribution histogram of OA-capped AgNPs. Adapted with permission from ref. [74]. Copyright 2016 American Chemical Society.

Synthesis of $\sim 35 \mathrm{~nm} \mathrm{SiO}_{2}$ with reverse microemulsion method

IGEPAL $^{\circledR}$ CO-520 (31.3 g) and cyclohexane (250 mL) were mixed together under stirring for $10 \mathrm{~min}$. Ammonia $(32 \%, 2.3 \mathrm{~mL})$ was then dropwise added to the solution and the mixture was allowed to be stirred 
for another 30 min before the addition of TEOS $(1.72 \mathrm{~mL})$. The reaction proceeded for approximately 16 hours at room temperature under vigorous stirring. The reaction was quenched by the addition of a large excess of ethanol ( $\sim 50 \mathrm{~mL})$, the $\mathrm{SiO}_{2}$-NPs were purified by three-fold centrifugation cycles in ethanol $(5000 \mathrm{rpm}, 30 \mathrm{~min})$. It is worth mentioning that tipsonicator is applied here to re-disperse the $\mathrm{SiO}_{2}$-NPs after centrifuge. The product was dried in vacuo.

This protocol provides uniform $\mathrm{SiO}_{2}$-NPs with a diameter of $38 \pm 1 \mathrm{~nm}$.
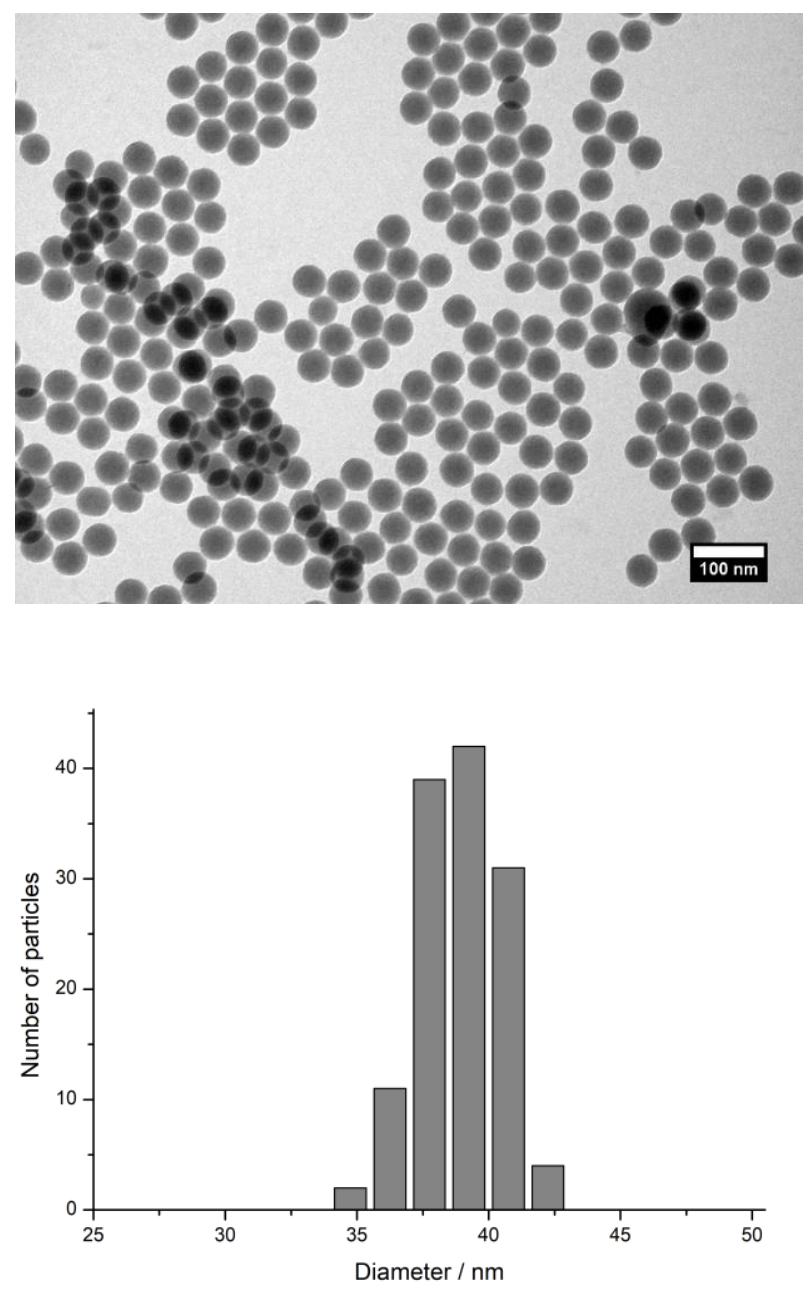

Figure 7.3: Exemplary TEM images and size distribution histogram of $\mathrm{SiO}_{2}$-NPs. 


\section{Optimized synthesis of $\sim 4.5 \mathrm{~nm}$ Brust-Schiffrin AuNPs}

This protocol was optimized from the method reported by Schiffrin et al.[62] Hydrogen tetrachloroauratetrihydrate $(94.5 \mathrm{mg}, 0.240 \mathrm{mmol})$ in nanopure water $(20 \mathrm{~mL})$ and tetra-octylammoniumbromide $(524.9 \mathrm{mg}$, $0.960 \mathrm{mmol}$ ) in toluene $(20 \mathrm{~mL}$ ) were mixed in two PP centrifuge tubes (10 $+10 \mathrm{ml}$ for each tube) and shaken vigorously for $120 \mathrm{~s}$. The organic layer was then removed carefully with a $5 \mathrm{ml}$ pipette and transferred to an unused disposable flat-bottom glass flask (100 $\mathrm{mL}$ with crimp neck). A freshly prepared solution of sodium borohydride $(72.6 \mathrm{mg}, 1.92 \mathrm{mmol})$ in water (5 $\mathrm{mL}$ ) was quickly added to the flask under vigorously stirring (1200 rpm) with a tiny stir bar (length $<1 \mathrm{~cm}$ ) on a digital stirrer. The stirring velocity (1200 rpm) was kept for $120 \mathrm{~s}$ and then slowed down to $300 \mathrm{rpm}$ so that organic and aqueous phases are not turbulently mixed. The mixture was stirred for a further 4 hours. Occasionally, some black gold settles down in the aqueous phase. In this case, the contact of the aggregated material with the organic phase should be avoided. The organic layer was then carefully moved to a separator funnel (cleaned with aqua regia and nanopure water) and washed with dilute sulphuric acid $(5 \%, 10 \mathrm{~mL})$ and water $(5 \times 10 \mathrm{~mL})$ and then dried over magnesium sulfate. $\mathrm{The}^{\mathrm{MgSO}} 4$ was then removed by syringe filtration. The AuNPs should be freshly used after synthesis. 


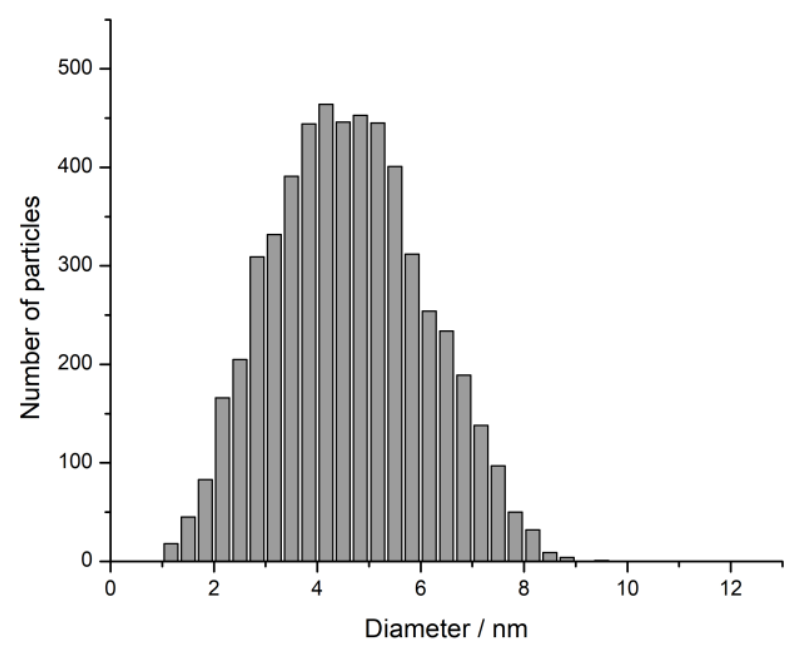

Figure 7.4: Size distribution histogram of $\sim 4.5 \mathrm{~nm}$ AuNPs from modified BrustSchiffrin synthesis.

\subsection{Synthesis of chemicals and polymerization's condition}

\section{Synthesis of activated RAFT (activated CDSPA)}

The synthesis and purification of the activated RAFT is described in literature ${ }^{[163]}$. The structure was confirmed by NMR spectroscopy. ${ }^{1} \mathrm{H}$ NMR $\left(300 \mathrm{MHz}, \mathrm{CDCl}_{3}\right): \delta(\mathrm{ppm}) 4.58(\mathrm{t}, 2 \mathrm{H}, J=7.5 \mathrm{~Hz}), 3.70-3.46(\mathrm{~m}, 2 \mathrm{H}), 3.33$ $(\mathrm{t}, 4 \mathrm{H}, J=7.5 \mathrm{~Hz}), 2.70-2.43(\mathrm{~m}, 2 \mathrm{H}), 1.89(\mathrm{~s}, 3 \mathrm{H}), 1.70$ (quint, $2 \mathrm{H}, J=7.5$ $\mathrm{Hz}), 1.43-1.26(\mathrm{~m}, 18 \mathrm{H}), 0.89(\mathrm{t}, 3 \mathrm{H}, J=6.6 \mathrm{~Hz})$.

Introducing $\mathrm{NH}_{2}$ group to the surface of $\mathrm{SiO}_{2}-\mathrm{NPs}$

In a typical experiment, $\mathrm{SiO}_{2}$-NPs (300 mg) were dispersed in dried THF (30 $\mathrm{mL}$ ) by 20 mins of bath sonicating. 3-Aminopropyldimethylethoxysilane $(150 \mu \mathrm{L})$ was then added to the sol. The reaction's mixture was kept overnight at $85{ }^{\circ} \mathrm{C}$ under stirring in an argon atmosphere to ensure an efficient anchoring of the amine group. After the reaction, the amine- 
functionalized $\mathrm{SiO}_{2}$-NPs were purified by three-fold of centrifugation cycles (THF/n-hexane) and dried under reduced pressure.

\section{Introducing RAFT group to the surface of $\mathrm{SiO}_{2}-\mathrm{NPs}$}

The amine-functionalized $\mathrm{SiO}_{2}$-NPs $(300 \mathrm{mg}$ ) was dispersed in $6 \mathrm{~mL}$ dried THF with the aid of bath sonication (20 min). A THF solution (3 ml) containing activated RAFT agents (activated CDSPA, $60 \mathrm{mg}$ ) was added to the sol and the mixture was sealed and kept stirring overnight. Three centrifugation cycles (THF/diethyl ether) was applied here to obtain the RAFT anchored $\mathrm{SiO}_{2}$-NPs. These particles could be dried in vacuo and stored in ambient conditions over at least 1 year without affecting their performance during polymerization.

This protocol was also applied on the $\mathrm{MNP@SiO}_{2}$ particles under identical condition.

\section{Surface-initiated polymerization on RAFT functionalized $\mathrm{SiO}_{2}-\mathrm{NPs}$}

RAFT capped $\mathrm{SiO}_{2}$-NPs were dispersed in a dioxane solution containing predetermined NiPAAM (Table 7.1 for $\mathrm{SiO}_{2}-\mathrm{NP}$ and Table 7.2 for $\mathrm{SiO}_{2}-\mathrm{NP}$ with magnetite core) in a polymerization tube. The tube is then bathsonicated for $40 \mathrm{~min}$ for a sufficient dispersion. From our experiments, dioxane was found to be the only suitable solvent for grafting poly(NiPAAM) from the surface of RAFT functionalized $\mathrm{SiO}_{2}$-NPs. It's worth mentioning that the position of the tubes must be adjusted during the sonication process to ensure sufficient cavitation in the sol. The RAFT agents (CDSPA), AIBN were then added to the sol and the mixture was purged with argon for 10 minutes. The polymerization of NiPAAM proceeded at $60{ }^{\circ} \mathrm{C}$. After predetermined times, the polymerization reactions were stopped by cooling in an ice bath and exposing the mixtures to air. The poly(NiPAAM) capped $\mathrm{SiO}_{2}$ nanohybrid and free polymer were gathered together by three centrifugation cycles from acetone with diethyl ether. The poly(NiPAAM) 
capped $\mathrm{SiO}_{2}$ nanohybrid was then isolated from the free polymer by threefold centrifugation/dispersion cycles in acetone. The molecular weight distribution of the free polymers is shown in Figure 7.5

The polymerization's condition is rather simple with MMA. In an exemplary run, $3.00 \mathrm{~g}$ MMA was mixed with $2.9 \mathrm{~g}$ CDSPA, $1.2 \mathrm{mg}$ AIBN and $5 \mathrm{mg}$ of RAFT capped $\mathrm{SiO}_{2}$-NPs. The mixture was dispersed using the same protocol described above. The polymerization was proceeded for 19 hours at $60^{\circ} \mathrm{C}$. Same purification's condition can also be applied here.

Table 7.1: Conditions for polymerization of RAFT functionalized $\mathrm{SiO}_{2}$-NPs. Each reaction consists of $1 \mathrm{~g}$ of $\mathrm{N}$ PAAM monomer and $20 \mathrm{mg} \mathrm{SiO}{ }_{2}$-NPs.

\begin{tabular}{lllllll}
\hline Polymer & $\mathrm{A}$ & $\mathrm{B}$ & $\mathrm{C}$ & $\mathrm{D}$ & $\mathrm{E}$ & $\mathrm{F}$ \\
\hline$M_{\mathrm{N}} \mathrm{kg} / \mathrm{mol}$ & 28 & 43 & 53 & 63 & 87 & 270 \\
\hline$D$ & 1.2 & 1.1 & 1.2 & 1.2 & 1.1 & 1.8 \\
\hline Equiv. of RAFT & 1.00 & 1.00 & 1.00 & 1.00 & 1.00 & 1.00 \\
\hline Equiv. of NiPAAM & 600 & 1000 & 1000 & 1700 & 1700 & 5000 \\
\hline Equiv. of AIBN & 0.40 & 0.40 & 0.40 & 0.80 & 0.80 & 0.80 \\
\hline Equiv. of dioxane & 4000 & 6000 & 6000 & 8000 & 8000 & 8000 \\
\hline Reaction's time / h & 3 & 2.5 & 3 & 2.5 & 3 & 4 \\
\hline
\end{tabular}




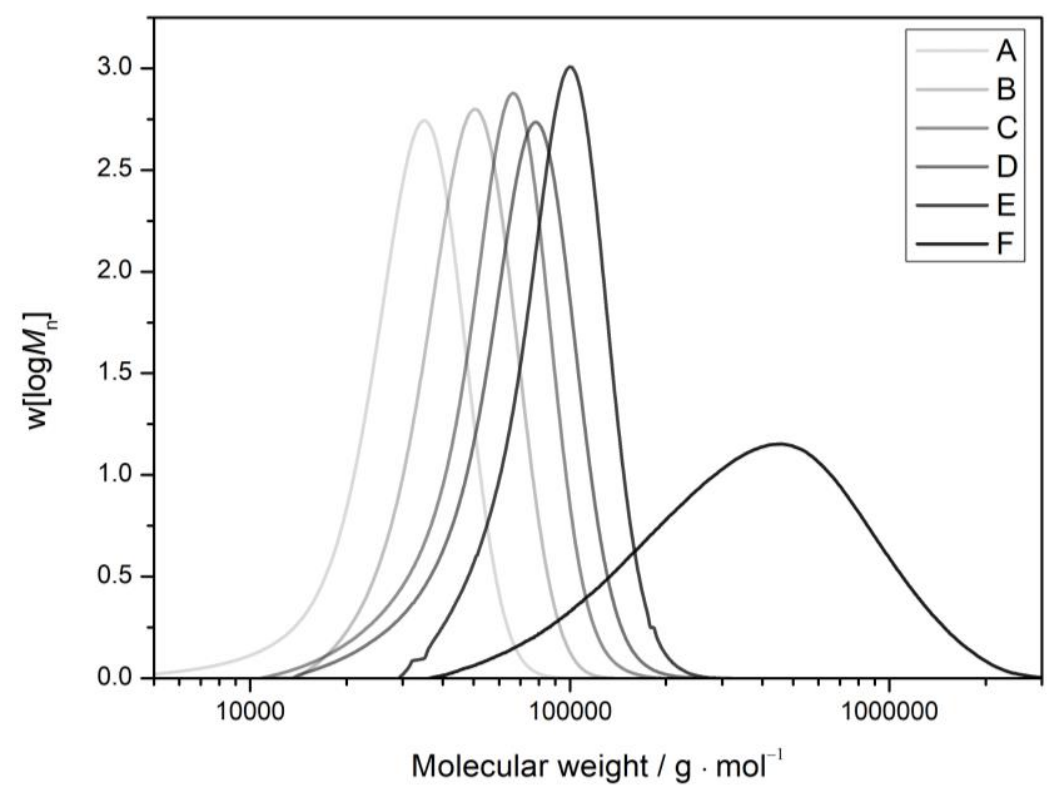

Figure 7.5: Molecular weight distributions from SEC analysis of the free polymers A-F (Table 7.1 / Table 5.1) for polymerization on $\mathrm{SiO}_{2}$.

Table 7.2: Polymerization conditions for the synthesis of the different poly(NiPAAM) grafted MNPs@SiO $2 \mathrm{I}-\mathrm{V}$ with results from SEC analysis for the corresponding free polymers. Adapted with permission from ref. [65]. Copyright 2018 John Wiley \& Sons, Inc.

\begin{tabular}{llllll}
\hline Polymer & $\mathrm{I}$ & $\mathrm{II}$ & $\mathrm{III}$ & $\mathrm{VI}$ & $\mathrm{V}$ \\
\hline$M_{\mathrm{n}} / \mathrm{kg} \cdot \mathrm{mol}^{-1}$ & 29 & 40 & 58 & 73 & 94 \\
\hline$D$ & 1.20 & 1.15 & 1.17 & 1.15 & 1.17 \\
\hline$m(\mathrm{MNP@SiO} 2) / \mathrm{mg}$ & 33 & 10 & 10 & 10 & 10 \\
\hline$m(\mathrm{RAFT}) / \mathrm{mg}$ & 3.12 & 4.50 & 3.10 & 2.10 & 2.10 \\
\hline Equiv. of RAFT & 1.00 & 1.00 & 1.00 & 1.00 & 1.00 \\
\hline$m($ NiPAAM) $/ \mathrm{mg}$ & 1000 & 750 & 700 & 1000 & 1000 \\
\hline Equiv. of NiPAAM & 1000 & 600 & 1000 & 1700 & 1700 \\
\hline$m($ AIBN) $/ \mathrm{mg}$ & 0.70 & 0.90 & 0.70 & 0.70 & 0.70 \\
\hline Eqiv. of AIBN & 0.40 & 0.50 & 0.40 & 0.80 & 0.80 \\
\hline$V($ dioxane $) / \mathrm{ml}$ & 4100 & 3750 & 3500 & 3500 & 3500 \\
\hline
\end{tabular}




\begin{tabular}{llllll}
\hline $\begin{array}{l}\text { Reaction's time / } \\
\text { min }\end{array}$ & 90 & 120 & 120 & 120 & 150 \\
\hline
\end{tabular}

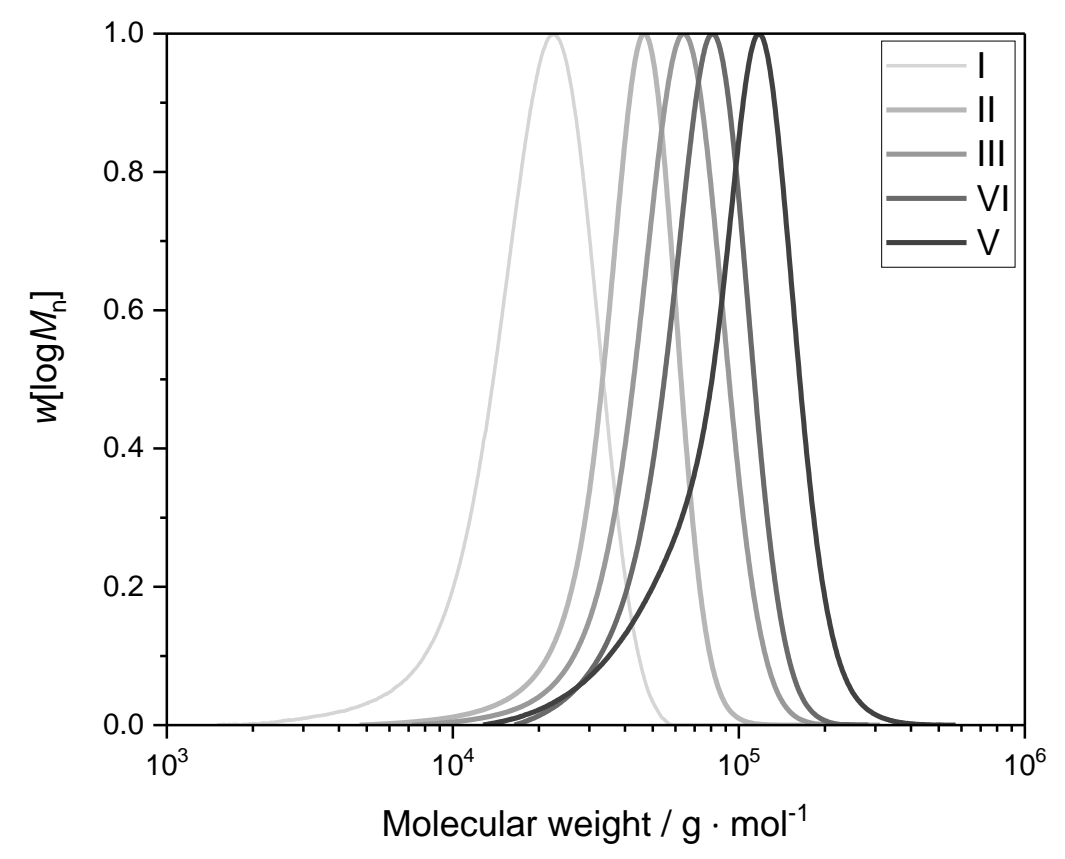

Figure 7.6: Molecular weight distributions from SEC analysis of the free polymers I-V

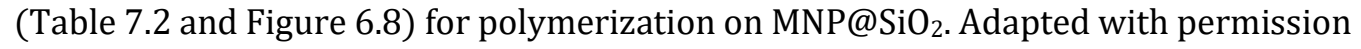
from ref. [65]. Copyright 2018 John Wiley \& Sons, Inc.

\section{Synthesis of Star and linear poly(NiPAAM)}

4-Arm star or linear RAFT agent, AIBN, NiPAAM, and DMF were mixed in polymerization tubes and the reaction mixtures were purged with argon for 15 minutes. The polymerization of NiPAAM was carried out at $60{ }^{\circ} \mathrm{C}$. After predetermined times, the polymerization reactions were stopped by cooling in an ice bath and exposing the mixtures to air. The polymer was isolated by threefold precipitation from acetone in diethyl ether. Detailed polymerization conditions are given in Table 7.3. The molecular weight distribution of the star polymers is shown in Figure 7.7. 
Table 7.3: Polymerization conditions for the synthesis of the different polymer samples and results from SEC analysis. Sample Star-II and Star-IV were previously used in the earlier publication [41]. Adapted with permission from ref. [74]. Copyright 2016 American Chemical Society.

\begin{tabular}{cccccc}
\hline Polymer & Star-I & Star-III & Star-V & Star-VI & Linear \\
\hline$M_{\mathrm{N}}$ g / mol & $29 \mathrm{k}$ & $44 \mathrm{k}$ & $60 \mathrm{k}$ & $78 \mathrm{k}$ & $30 \mathrm{k}$ \\
\hline$D$ & 1.1 & 1.1 & 1.1 & 1.1 & 1.1 \\
\hline Equiv. of RAFT & 1.00 & 1.00 & 1.00 & 1.00 & 1.00 \\
\hline $\begin{array}{c}\text { Equiv. of } \\
\text { NiPAAM }\end{array}$ & 400 & 400 & 1000 & 1000 & 400 \\
\hline Equiv. of AIBN & 0.40 & 0.40 & 0.50 & 0.50 & 0.25 \\
\hline $\begin{array}{c}\text { Equiv. of DMF } \\
\text { Reaction's } \\
\text { time / h }\end{array}$ & 1600 & 1600 & 6000 & 6000 & 1200 \\
\hline
\end{tabular}

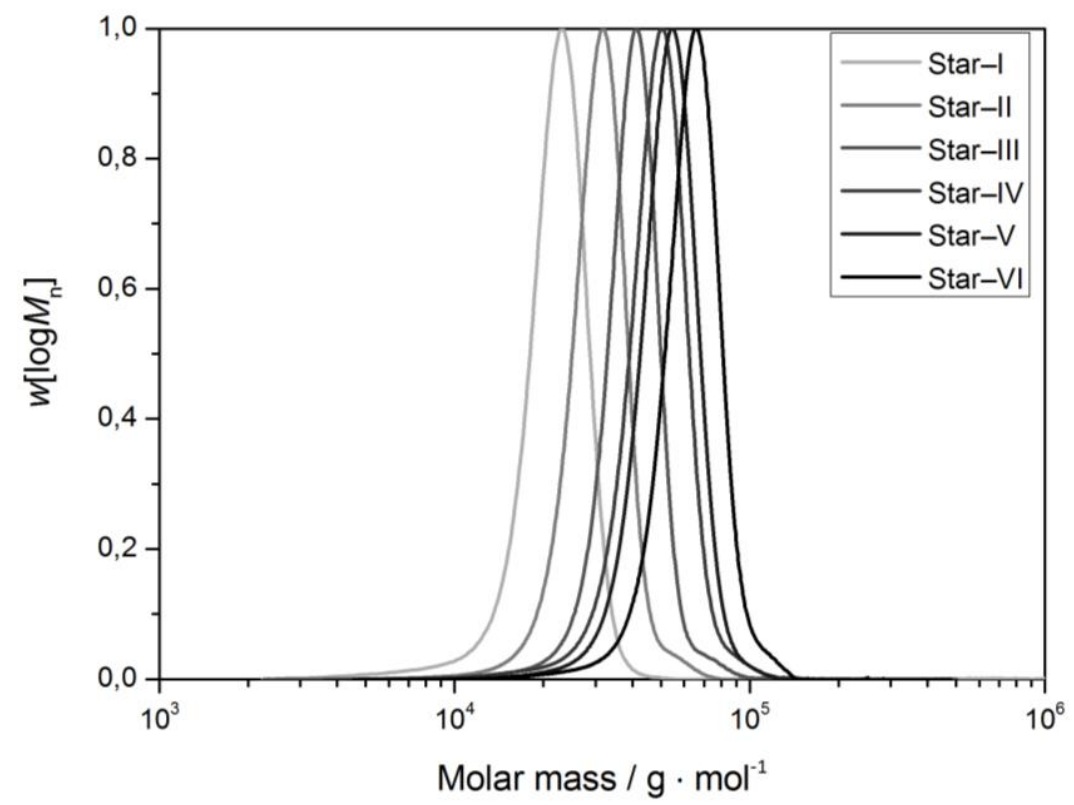

Figure 7.7: Molecular weight distribution from SEC analysis of the star polymers I-VI. Adapted with permission from ref. [74]. Copyright 2016 American Chemical Society. 


\title{
7.5 Grafting polymer onto AuNPs and AgNPs
}

\author{
Functionalization of citrate-capped AuNPs with star \\ $\operatorname{poly}\left(\mathrm{Ni}^{\mathrm{P} A A M}\right)$
}

In a typical experiment, star poly(NiPAAM) $(20 \mathrm{mg}$, dissolved in $5 \mathrm{ml}$ acetone) was added to a colloidal aqueous dispersion of citrate-capped $\sim 13 \mathrm{~nm}$ AuNPs (15 mL, approximately $0.1 \mathrm{mg} / \mathrm{mL}, 7.5 \mathrm{nmol} / \mathrm{L}$ ) under sonication at room temperature. The mixture was incubated overnight at room temperature and then centrifuged $(15300 \mathrm{G}, 1 \mathrm{~h})$ to remove the excess polymer and sodium citrate. The segregated material was redispersed in methanol $(20 \mathrm{~mL})$ and two additional centrifugation-redispersion steps were undertaken in order to exchange the solvent with $\mathrm{CHCl}_{3}$.

Functionalization of PE capped AuNPs and AgNPs

Trithiocarbonate terminated polyethylene or thiol terminated polyethylene $(2 \mathrm{mg})$ was dissolved in toluene $(1 \mathrm{~mL})$ at $90^{\circ} \mathrm{C}$. Toluene colloid containing AuNPs or AgNPs ( $2 \mathrm{~mL}, \sim 2 \mathrm{mg} / \mathrm{mL}$ ) was added to the PE solution and stirred for $30 \mathrm{~min}$ at $90^{\circ} \mathrm{C}$. The solution was used without purification for further usage.

\subsection{Self-assembly of planet-satellite nano-structures}

\section{Self-assembly of gold-planet-silver-satellite nano- structures}

$2 \mathrm{~mL}$ chloroform sol of OA-capped AgNPs containing approximately $3 \mathrm{mg}$ of AgNPs slowly mixed with $5 \mathrm{~mL}$ chloroform sol with approximately $0.3 \mathrm{mg}$ star RAFT poly(NiPAAM) capped AuNPs. The mixture was incubated for 30 minutes before $20 \mathrm{mg}$ of linear RAFT-poly(NiPAAM, $M_{\mathrm{N}}=40 \mathrm{~kg} / \mathrm{mol}$ ) was added to the mixture. The mixture was incubated further for $30 \mathrm{~min}$. The heavier planet-satellite assemblies were separated from the lighter 
excessive AgNPs and linear RAFT-polymer via three centrifugationredispersion cycles (2150 G, $1 \mathrm{~h})$.

\section{Self-assembly of silica-planet-gold-satellite nano- structures with citrate-capped $\sim 13$ nm AuNPs}

In a typical run, $4 \mathrm{mg}$ of poly(NiPAAM) capped $\mathrm{SiO}_{2}-\mathrm{NPs}$ (planet NPs) was dispersed in $40 \mathrm{ml}$ ethanol with the aid of bath-type ultrasonication (5 min). $10 \mathrm{ml}$ colloid of citrate-capped AuNPs ( $\sim 0.1 \mathrm{mg} / \mathrm{ml}$ ) was quickly added to the ethanol colloid containing planet NPs under sonication. The mixture was allowed to incubate for $20 \mathrm{~min}$ before the addition of $0.5 \mathrm{ml}$ of linear poly(NiPAAM) solution $\left(M_{\mathrm{n}}=40 \mathrm{~kg} / \mathrm{mol}, 1 \mathrm{mg} / \mathrm{ml}\right) .60 \mathrm{ml}$ water was then quickly added to the colloid and the mixture was allowed to rest for another $30 \mathrm{~min}$.

The mixture was then subjected to centrifugation (6000 rpm, $1 \mathrm{~h}$ ) to remove the excess of sodium citrate and linear polymer. After the centrifugation, the planet-satellite nanostructures were collected on the wall of the centrifugation tube. The tube was then dried by argon flow for 20 min to remove the residual water. The nanostructures were finally dispersed using $2 \mathrm{ml} \mathrm{CHCl}_{3}$ with the aid of sonication ( $\left.10 \mathrm{~min}\right)$ and stored at $6{ }^{\circ} \mathrm{C}$.

\section{Self-assembly of silica-planet-gold-satellite nano- structures with TOAB-capped $\sim 5$ nm AuNPs}

In a typical run, $0.3 \mathrm{mg}$ of poly(NiPAAM) capped $\mathrm{SiO}_{2}$-NPs (planet NPs) was dispersed in $3 \mathrm{ml} \mathrm{CHCl}_{3}$ with the aid of bath-type ultrasonication (5 min). $40 \mu$ l colloid of citrate-capped AuNPs ( 1 mg / ml) was quickly added to the planet NPs' colloid under sonication. The mixture was allowed to incubate for $1 \mathrm{~min}$ before the addition of $0.5 \mathrm{ml}$ of linear poly(NiPAAM) solution $\left(M_{\mathrm{n}}=40 \mathrm{~kg} / \mathrm{mol}, 1 \mathrm{mg} / \mathrm{ml}\right)$. 


\title{
7.7 Preparation and experiments of planet-satellite nanostructure decorated $\mathrm{CaF}_{2}$ substrate
}

\author{
Formation of the monolayer of the planet-satellite \\ nanostructure on $\mathrm{CaF}_{2}$ crystal
}

$\mathrm{CaF}_{2}$ crystal with the dimension of $1 \times 1 \times 0.1 \mathrm{~cm}$ (both sides highly polished) was used as the substrate for the binary planet-satellite nanostructures. First, the $\mathrm{CaF}_{2}$ crystal was rinsed with acetone (p. A) several times and dried by argon flow. The substrate was then placed on a glass petri dish and subjected to $\mathrm{O}_{2}$ plasma treatment. The plasma cleaning proceeded for 10 min with the power of $100 \mathrm{~W}$. The plasma-treated $\mathrm{CaF}_{2}$ substrate was immediately taken to the dip-coating process. The detailed conditions for dip-coating are listed in section 5.4.1. The planet-satellite nanostructure decorated $\mathrm{CaF}_{2}$ crystal can be stored under ambient conditions for months without observable change.

\section{Transfer of planet-satellite nanostructure from $\mathrm{CaF}_{2}$ onto hydrogel}

The planet-satellite nanostructure decorated $\mathrm{CaF}_{2}$ substrate was placed on a glass petri dish in the plasma oven. The $\mathrm{O}_{2}$ plasma etching was conducted in 2 min interval for 5 times with 1 min pause between each phase with a power of $100 \mathrm{~W}$.

After the plasma cleaning, the substrate was placed in a $\mathrm{CHCl}_{3}$ solution containing $1 \mathrm{mM} \mathrm{BAC}$ and $100 \mathrm{mM}$ APTOS and allowed to rest overnight. After the incubation, the substrate was rinsed with clean $\mathrm{CHCl}_{3}$ several times and dried under argon flow. The substrate was then placed into a Teflon mold with an indentation of $1.3 \times 1.3 \times 0.3 \mathrm{~cm}$. A solution consists of $50 \%$ PEG-diacrylate $\left(M_{\mathrm{n}}=700 \mathrm{~g} / \mathrm{mol}\right), 50 \%$ water and HHMPP $(1 \mathrm{mg} / \mathrm{ml})$ was added to the mold and the photopolymerization was proceed for $3 \mathrm{~min}$ under UV radiation in a UV curing chamber. The hydrogel was then 
incubated in water for $1 \mathrm{~h}$ to remove any unreacted monomer and initiator. The $\mathrm{CaF}_{2}$ substrate can be removed gently using a tweezer.

\section{Passivation of $\mathrm{CaF}_{2}$ substrate}

The $\mathrm{CaF}_{2}$ (whether with or without nanoparticles) substrate was placed on a glass Petri dish and plasma-treated for $10 \mathrm{~min}$ at $100 \mathrm{~W}$. After that, the $\mathrm{CaF}_{2}$ substrate was immediately placed on a droplet containing $0.25 \mathrm{mg} / \mathrm{ml}$ PLL-g-PEG for 40 minutes and then rinsed with water. After drying with argon flow, the sample is taken for biological experiments within 20 min.

\section{Testing passivation of $\mathrm{CaF}_{2}$ substrate for RGD}

The $\mathrm{CaF}_{2}$ (whether with or without nanoparticles) substrate was placed on a glass petri dish and plasma-treated for $10 \mathrm{~min}$ at $100 \mathrm{~W}$. After that, the $\mathrm{CaF}_{2}$ substrate was immediately placed on a droplet containing $0.25 \mathrm{mg} / \mathrm{ml}$ PLL-g-PEG for 40 minutes and rinsed with water. The substrate was then placed on another droplet containing $5 \mu \mathrm{M}$ RGD solution and incubated for $2 \mathrm{~h}$. After rinsing with water and drying with argon flow, the sample is taken for biological experiment within $20 \mathrm{~min}$. 


\section{Chapter 8 Closing Remarks}

\subsection{Conclusions}

Within this thesis, the state-of-the-art in the realm of fabrication strategy and applications of the multifunctional planet-satellite nanohybrids have been reviewed in Chapter 1 . The building blocks and tools for the fabrication of binary planet-satellite nanoassemblies were discussed in Chapter 2 in detail including the properties of each nanocomponent and the strategy for the functionalization of the nanoparticles with polymer. Since the silicaplanet-gold-satellite nanostructure was designed for the cell-response experiment, Chapter 3 gave an introduction focusing on the methodology and the current challenges of the state-of-the-art nanopatterning mediated cell-responses experiment in-depth.

The fabrication strategies and the hybrid properties of RAFT polymer mediated gold-planet-silver-satellite (Chapter 4) and silica-planet-goldsatellite (Chapter 5) were studied in detail. The monolayer formation of the silica-planet-gold-satellite nanostructure on $\mathrm{CaF}_{2}$ was established by applying dip-coating method. Due to the limited passivation on the $\mathrm{CaF}_{2}$ surface, the planet-satellite nanostructures were transferred from $\mathrm{CaF}_{2}$ to hydrogel surface. Chapter 6 expended the functionality of the RAFT polymer-based nanohybrids by changing the polymer or introducing further nanoparticles, respectively. Polyethylene capped AuNPs and AgNPs was fabricated using a straightforward self-assembly process. Using the

reversed microemulsion technique, magnetite nanoparticles was introduced in the silica nanoparticles carrying its superparamagnetic property.

The fabrications strategy of the planet-satellite nanostructure using RAFT polymer is highly flexible allowing the simultaneous integration of the properties from various nanoparticles (AuNPs, AgNPs, magnetite NPs, $\mathrm{SiO}_{2}$ - 
NPs) and functional polymers (e.g., stimuli-response and enhanced dispersibility in the desired environment). Figure 8.1 demonstrates the framework to customize a planet-satellite nanostructure for desired hybrid functions. Furthermore, the dense polymer brush linker between the planet and the satellite nanoparticles enabled a precise control over the interparticle distance in the range from 5 to $50 \mathrm{~nm}$ which further enables the fine-tuning of the interaction between the nanoparticles.

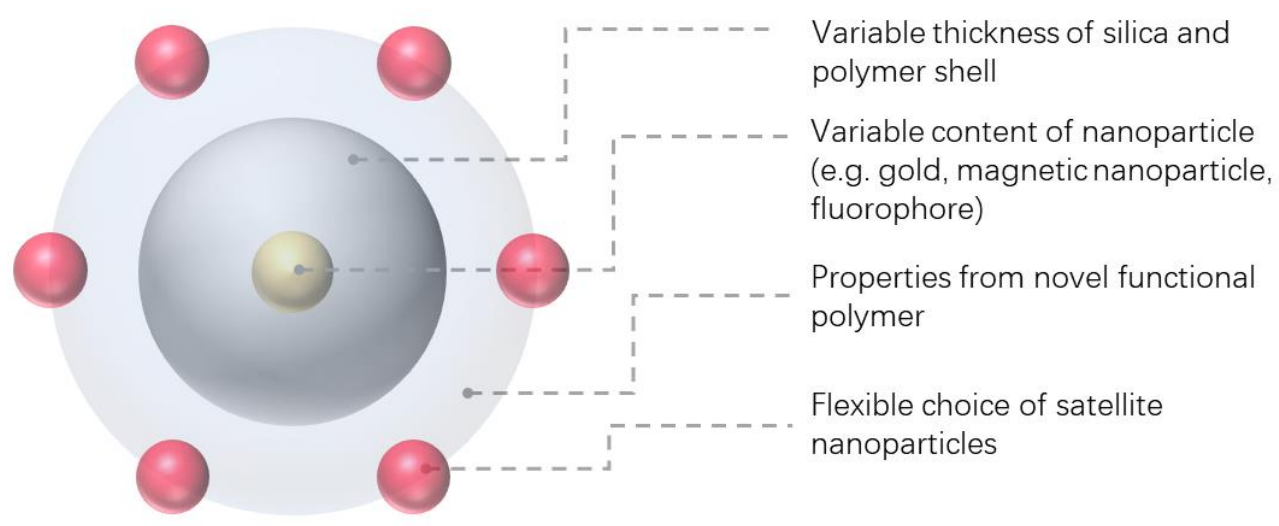

Figure 8.1: Schematic representation of the flexible framework of the planet-satellite nanostructure accessible using the methods established in this thesis.

The initial designed application of the silica-planet-gold-satellite nanostructure was to use the binary pattern as a template for the cellresponse experiment. During this thesis, the reliable synthesis route was established for different interparticle distances. $\mathrm{CaF}_{2}$ was proved to be a suitable surface for the formation of monolayer and serves as the substrate with neutral chemical reactivity. However, from cell experiments, the PEGylation on the $\mathrm{CaF}_{2}$ surface did not provide sufficient passivation for peptide. To address this issue, a method for transferring both AuNPs and $\mathrm{SiO}_{2}$-NPs was developed. The whole experiment protocol, e.g., the fabrication of each nanoparticle, each modification/polymerization steps and procedure of surface coating, were highly optimized to have high 
experimental reproducibility and sufficient capability to produce samples substrate in a number of tens.

\subsection{Future perspectives}

On the binary nanopattern induced cell-response experiments, the next steps include the identifying the surface structure of nanopatterned hydrogel and optimizing the process of orthogonal functionalization of peptides on both nanoparticles.

SEM is reported as a suitable method for the surface analysis of hydrogel. However, due to the water content and non-conducting nature of the hydrogel, the SEM experiment becomes very challenging. The samples must be dried carefully before placed into SEM which operates under high vacuum condition. A nano-thin conductive coating is also crucial here to enable the SEM imaging with reasonable resolution. The coating material (e.g., gold, carbon) and its thickness must be optimized to resolve the nanoparticles without covering up the surface structure of hydrogel. 


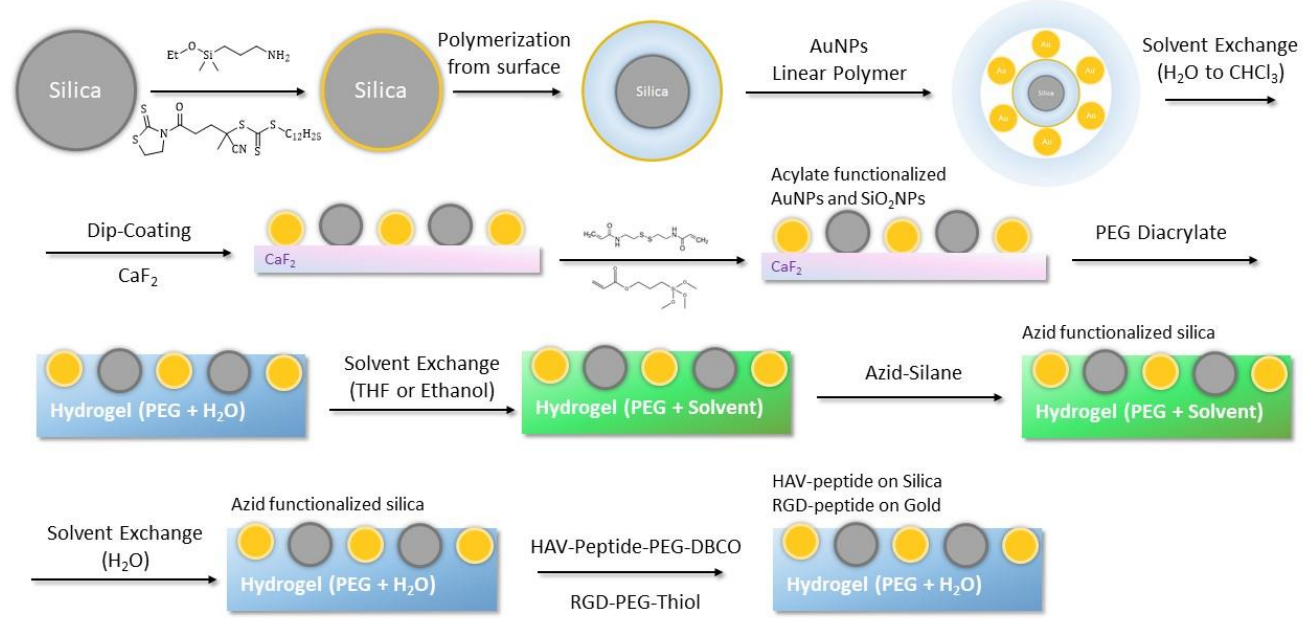

Figure 8.2: Fabrication of binary planet-silica nanoparticles decorated hydrogel surface, Including a proposal route $\left(3^{\text {rd }}\right.$ and $4^{\text {th }}$ line) for binding two distinct peptides (RGD-peptide for AuNPs and HAV-peptide for $\mathrm{SiO}_{2}$-NPs).

The orthogonal bio-labeling on both AuNPs and $\mathrm{SiO}_{2}$-NPs must be carried out considering the chemistry environments of the whole system including the hydrogel, rest water content in the hydrogel, solvent and both nanoparticles. For AuNPs, the protocol is well established by employing cysteine (contains thiol group) units at the chain termini as anchoring moiety. For $\mathrm{SiO}_{2}$-NPs, a reliable method must be developed. Since the silane group is sensitive to moisture, a two-step approach including the application of "click-chemistry," will be more favored in this case. Since the peptide labels are water-soluble, the clicking group should be highly water-resistant, which is also convenient for the storage. Under this context, the copper-free click chemistry with dibenzocyclooctyne (DBCO) + azide ${ }^{[164]}$ should be a suitable choice for this task. According to the demand of our cooperation partner from group Schön (university clinic of Göttingen), the following DBCO functionalized HAV tagged peptide is purchased: Ac-HAVDIGGG(PEG5)3-Lys(DBCO)-amid. The experiment will be done with the first functionalization of silica with azide-silane (e.g., 3- 
azidopropyltriethoxysilane) in a water-free state and then bring RGDpeptide (for AuNPs) and HAV-peptide(for $\mathrm{SiO}_{2}$-NPs) to the corresponding particles. It is worth mentioning that the solvent exchange should be necessary due to the unfavored reaction between silane and water.

Insofar, interdisciplinary collaborations are critical to optimizing the conditions interactively and will always play the central role in overcoming the raised questions as the project proceed. Further intensive studies on silica-planet-gold-satellite templated binary nanopattern for inducing cellresponse experiment will bring exciting results and new insights into the realm of dermatology. 


\section{Abbreviations and Symbols}

2D

3D

AFM

AIBN

appx.

APTES

APTOS

BAC

CDSPA

CT

DBCO

$d$

DLS

$\bigoplus$

DMAc

DMF

DNA

e.g.

equiv. two-dimensional

three-dimensional

atomic force microscopy

azobisisobutyronitrile

approximately

(3-aminopropyl)triethoxysilane

3-acryloxypropyltrimethoxysilane

N,N'-bis(acryloyl)cystamine

4-cyano-4-(dodecylsulfanyl-thio-carbonyl)-

sulfanylpentanoic

computed tomography

dibenzocyclooctyne

diameter

dynamic light scattering

dispersity

dimethylacetamide

dimethylformamide

deoxyribonucleic acid

lat.: exempli gratia

equivalent 


$\begin{array}{ll}\text { et al. } & \text { lat.: et alii } \\ \text { FC } & \text { field cooled } \\ \text { HAADF } & \text { high-angle annular dark-field } \\ \text { HBP } & \text { hyberbranched polymer } \\ \text { HHMPP } & \text { 2-hydroxy-4'-(2-hydroxyethoxy)-2- } \\ & \text { methylpropiophenone } \\ \text { HPLC } & \text { high performance liquid chromatography } \\ \text { HRTEM } & \text { high resolution transmission electron } \\ \text { i.e. } & \text { microscopy } \\ M_{\text {S }} & \text { lat.: id est } \\ \text { MBA } & \text { specific saturation magnetization } \\ \text { MMA } & \text { 4-mercaptobenzoic acid } \\ M_{N} & \text { methyl methacrylate } \\ \text { MNP } & \text { average molecular weight } \\ \text { MOF } & \text { magnetit nanoparticle } \\ \text { NiPAAM } & \text { metal organic framework } \\ \text { NIR } & \text { N-isopropylacrylamide } \\ \text { NMR } & \text { near-infrared } \\ \text { NP } & \text { nuclear magnetic resonance } \\ \text { OA } & \text { nanoparticle } \\ \text { p.a. } & \text { oleylamine } \\ \text { PBS } & \text { pro analysi } \\ & \text { phosphate-buffered saline } \\ & \end{array}$




$\begin{array}{ll}\text { PE } & \text { polyethene } \\ \text { PEG } & \text { polyethylene glycol } \\ \text { PLL } & \text { poly-l-lysine } \\ \text { PLL-g-PEG } & \text { poly-l-lysine-graft-peg } \\ \text { PP } & \text { polypropylene } \\ \text { PTFE } & \text { polytetrafluoroethylene } \\ \text { RAFT } & \text { reversible addition-fragmentation chain- } \\ \text { ref } & \text { transfer } \\ \text { RNA } & \text { reference } \\ \text { rpm } & \text { ribonucleic acid } \\ \text { rt } & \text { revolutions per minute } \\ \text { SEC } & \text { room temperature } \\ \text { SEM } & \text { size-exclusion chromatography } \\ \text { SER } & \text { scanning electron microscopy } \\ \text { SPR } & \text { surface-enhanced Raman } \\ \text { SQUID } & \text { surface plasmon resonance } \\ \text { STEM } & \text { superconducting quantum interference device } \\ T_{B} & \text { tetraethyl orthosilicate } \\ \text { TE } & \text { thermogravimetric analysis } \\ \text { TEM } & \text { blocking temperature } \\ \text { TEOS } & \text { transmission electron } \\ \text { TGA } & \text { transmission electron microscopy } \\ & \end{array}$




$\begin{array}{ll}\text { THF } & \text { tetrahydrofuran } \\ \text { TOAB } & \text { tetrabutylammonium bromide } \\ \text { TTC } & \text { trithiocarbonate } \\ \text { UCNP } & \text { upconverting nanoparticle } \\ \text { UV } & \text { ultraviolet } \\ \text { Vis } & \text { visible } \\ \text { w/o } & \text { water-in-oil } \\ \text { ZFC } & \text { zero field cooled } \\ \delta & \text { chemical shift }\end{array}$

Only for amino acid:

$\begin{array}{ll}\text { A } & \text { alanine } \\ \text { Ac } & \text { acid group as end-group } \\ \text { D } & \text { aspartic acid } \\ \text { G } & \text { glycine } \\ \text { H } & \text { histidine } \\ \text { I } & \text { isoleucine } \\ \text { Lys } & \text { Lysine } \\ \text { R } & \text { arginine } \\ \text { V } & \text { valine }\end{array}$




\section{Bibliography}

[1] Brust, M.; Bethell, D.; Kiely, C. J.; Schiffrin, D. J. Self-assembled gold nanoparticle thin films with nonmetallic optical and electronic properties. Langmuir 1998, 14, 54255429.

[2] Talley, C. E.; Jackson, J. B.; Oubre, C.; Grady, N. K.; Hollars, C. W.; Lane, S. M.; Huser, T. R.; Nordlander, P.; Halas, N. J. Surface-enhanced Raman scattering from individual Au nanoparticles and nanoparticle dimer substrates. Nano letters 2005, 5, 1569-1574.

[3] Shipway, A. N.; Katz, E.; Willner, I. Nanoparticle arrays on surfaces for electronic, optical, and sensor applications. chemphyschem 2000, 1, 18-52.

[4] Anker, J. N.; $\quad$ Hall, W. P.; $\quad$ Lyandres, O.; Shah, N. C.; Zhao, J.; Van Duyne, R. P. Nanoscience and Technology: A Collection of Reviews from Nature Journals, World Scientific, 2010; pp 308-319.

[5] Lopez, N.; Janssens, T.; Clausen, B.; Xu, Y.; Mavrikakis, M.; Bligaard, T.; Nørskov, J. K. On the origin of the catalytic activity of gold nanoparticles for low-temperature CO oxidation. Journal of Catalysis 2004, 223, 232-235.

[6] Linic, S.; Christopher, P.; Ingram, D. B. Plasmonic-metal nanostructures for efficient conversion of solar to chemical energy. Nature materials 2011, 10, 911.

[7] McGilvray, K. L.; Decan, M. R.; Wang, D.; Scaiano, J. C. Facile photochemical synthesis of unprotected aqueous gold nanoparticles. Journal of the American Chemical Society 2006, 128, 15980-15981.

[8] Kruss, S.; Wolfram, T.; Martin, R.; Neubauer, S.; Kessler, H.; Spatz, J. P. Stimulation of cell adhesion at nanostructured teflon interfaces. Advanced Materials 2010, 22, 54995506.

[9] Hainfeld, J.; Slatkin, D.; Focella, T.; Smilowitz, H. Gold nanoparticles: a new X-ray contrast agent. The British journal of radiology 2006, 79, 248-253.

[10] Ghosh, P.; Han, G.; De, M.; Kim, C. K.; Rotello, V. M. Gold nanoparticles in delivery applications. Advanced drug delivery reviews 2008, 60, 1307-1315. 
[11] Arvizo, R. R.; $\quad$ Bhattacharyya, S.; $\quad$ Kudgus, R. A.; $\quad$ Giri, K.; $\quad$ Bhattacharya, R.; Mukherjee, P. Intrinsic therapeutic applications of noble metal nanoparticles: past, present and future. Chemical Society Reviews 2012, 41, 2943-2970.

[12] Kim, D. J.; Kang, S. M.; Kong, B.; Kim, W.-J.; Paik, H.-j.; Choi, H.; Choi, I. S. Formation of thermoresponsive gold nanoparticle/PNIPAAM hybrids by surface-initiated, atom transfer radical polymerization in aqueous media. Macromolecular Chemistry and Physics 2005, 206, 1941-1946.

[13] Xue, Y.; Li, X.; Li, H.; Zhang, W. Quantifying thiol-gold interactions towards the efficient strength control. Nature communications 2014, 5, 4348.

[14] Grönbeck, H.; Curioni, A.; Andreoni, W. Thiols and disulfides on the Au (111) surface: the headgroup- gold interaction. Journal of the American Chemical Society 2000, 122, 3839-3842.

[15] Blakey, I.; $\quad$ Schiller, T. L.; $\quad$ Merican, Z.; Fredericks, P. M. Interactions of phenyldithioesters with gold nanoparticles (AuNPs): implications for AuNP functionalization and molecular barcoding of AuNP assemblies. Langmuir 2009, 26, 692-701.

[16] Duwez, A.-S.; Guillet, P.; Colard, C.; Gohy, J.-F.; Fustin, C.-A. Dithioesters and trithiocarbonates as anchoring groups for the "grafting-to" approach. Macromolecules 2006, 39, 2729-2731.

[17] Ebeling, B.; Vana, P. RAFT-polymers with single and multiple trithiocarbonate groups as uniform gold-nanoparticle coatings. Macromolecules 2013, 46, 4862-4871.

[18] Li, Z.; Jin, R.; Mirkin, C. A.; Letsinger, R. L. Multiple thiol-anchor capped DNA-gold nanoparticle conjugates. Nucleic acids research 2002, 30, 1558-1562.

[19] Shimmin, R. G.; Schoch, A. B.; Braun, P. V. Polymer size and concentration effects on the size of gold nanoparticles capped by polymeric thiols. Langmuir 2004, 20, 56135620 .

[20] Gandra, N.; Abbas, A.; Tian, L.; Singamaneni, S. Plasmonic planet-satellite analogues: hierarchical self-assembly of gold nanostructures. Nano letters 2012, 12, 2645-2651.

[21] Gellner, M.; Steinigeweg, D.; Ichilmann, S.; Salehi, M.; Schütz, M.; Kömpe, K.; Haase, M.; Schlücker, S. 3D self-assembled plasmonic superstructures of gold nanospheres: Synthesis and characterization at the single-particle level. Small 2011, 7, 3445-3451. 
[22] Gunawidjaja, R.; $\quad$ Kharlampieva, E.; $\quad$ Choi, I.; $\quad$ Tsukruk, V.V. $\quad$ Bimetallic nanostructures as active Raman markers: gold-nanoparticle assembly on 1D and 2D silver nanostructure surfaces. small 2009, 5, 2460-2466.

[23] Choi, I.; Song, H. D.; Lee, S.; Yang, Y. I.; Kang, T.; Yi, J. Core-satellites assembly of silver nanoparticles on a single gold nanoparticle via metal ion-mediated complex. Journal of the American Chemical Society 2012, 134, 12083-12090.

[24] Weng, Z.; Wang, H.; Vongsvivut, J.; Li, R.; Glushenkov, A. M.; He, J.; Chen, Y.; Barrow, C. J.; Yang, W. Self-assembly of core-satellite gold nanoparticles for colorimetric detection of copper ions. Analytica chimica acta 2013, 803, 128-134.

[25] Gandra, N.; Singamaneni, S. "Clicked" plasmonic core-satellites: covalently assembled gold nanoparticles. Chemical Communications 2012, 48, 11540-11542.

[26] Zhu, T.; Fu, X.; Mu, T.; Wang, J.; Liu, Z. pH-dependent adsorption of gold nanoparticles on $p$-aminothiophenol-modified gold substrates. Langmuir 1999, 15, 5197-5199.

[27] Nuzzo, R. G.; Dubois, L. H.; Allara, D. L. Fundamental studies of microscopic wetting on organic surfaces. 1. Formation and structural characterization of a selfconsistent series of polyfunctional organic monolayers. Journal of the American Chemical Society 1990, 112, 558-569.

[28] Kankate, L.; Turchanin, A.; Gooelzhaeuser, A. On the release of hydrogen from the $\mathrm{S}$ - H groups in the formation of self-assembled monolayers of thiols. Langmuir 2009, 25, 10435-10438.

[29] Yoon, J. H.; Lim, J.; Yoon, S. Controlled assembly and plasmonic properties of asymmetric core-satellite nanoassemblies. ACS nano 2012, 6, 7199-7208.

[30] Yang, Y.; Zhu, J.; Zhao, J.; Weng, G.-J.; Li, J.-J.; Zhao, J.-W. A growth of spherical gold satellites on the surface of $\mathrm{Au} @ \mathrm{Ag} @ \mathrm{SiO}_{2}$ core-shell nanostructures used for an ultrasensitive SERS immunoassay of alpha-fetoprotein. ACS applied materials \& interfaces 2019,

[31] Rong, Z.; Xiao, R.; Wang, C.; Wang, D.; Wang, S. Plasmonic Ag core-satellite nanostructures with a tunable silica-spaced nanogap for surface-enhanced raman scattering. Langmuir 2015, 31, 8129-8137.

[32] Westcott, S. L.; Oldenburg, S. J.; Lee, T. R.; Halas, N. J. Formation and adsorption of clusters of gold nanoparticles onto functionalized silica nanoparticle surfaces. Langmuir 1998, 14, 5396-5401. 
[33] Chou, L. Y.; Zagorovsky, K.; Chan, W. C. DNA assembly of nanoparticle superstructures for controlled biological delivery and elimination. Nature nanotechnology 2014, 9, 148-155.

[34] Knop, K.; Hoogenboom, R.; Fischer, D.; Schubert, U. S. Poly (ethylene glycol) in drug delivery: pros and cons as well as potential alternatives. Angewandte chemie international edition 2010, 49, 6288-6308.

[35] Schreiber, R.; $\quad$ Do, J.; $\quad$ Roller, E.-M.; $\quad$ Zhang, T.; $\quad$ Schüller, V. J.; $\quad$ Nickels, P. C.; Feldmann, J.; Liedl, T. Hierarchical assembly of metal nanoparticles, quantum dots and organic dyes using DNA origami scaffolds. Nature nanotechnology 2014, 9, 74.

[36] Zhao, X.; Xu, L.; Sun, M.; Ma, W.; Wu, X.; Kuang, H.; Wang, L.; Xu, C. Gold-quantum dot core-satellite assemblies for lighting up microRNA in vitro and in vivo. Small2016, $12,4662-4668$.

[37] Qu, A.; Xu, L.; Sun, M.; Liu, L.; Kuang, H.; Xu, C. Photoactive hybrid AuNR-Pt@ $\mathrm{Ag}_{2} \mathrm{~S}$ core-satellite Nanostructures for Near-Infrared Quantitive Cell Imaging. Advanced Functional Materials 2017, 27, 1703408.

[38] Sun, M.; Xu, L.; Ma, W.; Wu, X.; Kuang, H.; Wang, L.; Xu, C. Hierarchical plasmonic nanorods and upconversion core-satellite nanoassemblies for multimodal imagingguided combination phototherapy. Advanced Materials 2016, 28, 898-904.

[39] He, L.; Brasino, M.; Mao, C.; Cho, S.; Park, W.; Goodwin, A. P.; Cha, J. N. DNAassembled core-satellite upconverting-metal-organic framework nanoparticle superstructures for efficient photodynamic therapy. Small 2017, 13, 1700504.

[40] Dey, P.; Zhu, S.; Thurecht, K. J.; Fredericks, P. M.; Blakey, I. Self-assembly of plasmonic core-satellite nano-assemblies mediated by hyperbranched polymer linkers. Journal of Materials Chemistry B 2014, 2, 2827-2837.

[41] Rossner, C.; Vana, P. Planet-satellite nanostructures made to order by RAFT star polymers. Angewandte Chemie International Edition 2014, 53, 12639-12642.

[42] Rossner, C.; Roddatis, V.; Lopatin, S.; Vana, P. Functionalization of planet-satellite nanostructures revealed by nanoscopic localization of distinct macromolecular species. Macromolecular rapid communications 2016, 37, 1742-1747.

[43] Rossner, C.; Tang, Q.; Glatter, 0.; Müller, M.; Vana, P. Uniform distance scaling behavior of planet-satellite nanostructures made by star polymers. Langmuir 2017, 33, 2017-2026. 
[44] Mueller, R.; Kammler, H. K.; Wegner, K.; Pratsinis, S. E. OH surface density of SiO2 and TiO2 by thermogravimetric analysis. Langmuir 2003, 19, 160-165.

[45] Zou, H.; Wu, S.; Shen, J. Polymer/silica nanocomposites: preparation, characterization, properties, and applications. Chemical reviews 2008, 108, 38933957.

[46] Li, C.; Benicewicz, B. C. Synthesis of well-defined polymer brushes grafted onto silica nanoparticles via surface reversible addition- fragmentation chain transfer polymerization. Macromolecules 2005, 38, 5929-5936.

[47] Wu, L.; Glebe, U.; Böker, A. Fabrication of thermoresponsive plasmonic coresatellite nanoassemblies with a tunable stoichiometry via surface-Initiated reversible addition-fragmentation chain transfer polymerization from silica nanoparticles. Advanced Materials Interfaces 2017, 4, 1700092.

[48] Rossner, C.; $\quad$ Glatter, O.; Vana, P. Stimulus-responsive planet-satellite nanostructures as colloidal actuators: reversible contraction and expansion of the planet-satellite distance. Macromolecules 2017, 50, 7344-7350.

[49] Han, F.; Soeriyadi, A. H.; Gooding, J.J. Reversible thermoresponsive plasmonic core-satellite nanostructures that exhibit both expansion and contraction (UCST and LCST). Macromolecular rapid communications 2018, 39, 1800451.

[50] Aslan, K.; Gryczynski, I.; Malicka, J.; Matveeva, E.; Lakowicz, J. R.; Geddes, C. D. Metal-enhanced fluorescence: an emerging tool in biotechnology. Current opinion in biotechnology 2005, 16, 55-62.

[51] Pompa, P.; $\quad$ Martiradonna, L.; $\quad$ Della Torre, A.; $\quad$ Della Sala, F.; $\quad$ Manna, L.; De Vittorio, M.; Calabi, F.; Cingolani, R.; Rinaldi, R. Metal-enhanced fluorescence of colloidal nanocrystals with nanoscale control. Nature nanotechnology 2006, 1, 126.

[52] Heskins, M.; Guillet, J. E. Solution properties of poly (N-isopropylacrylamide). Journal of Macromolecular Science-Chemistry 1968, 2, 1441-1455.

[53] Schmaljohann, D. Thermo-and pH-responsive polymers in drug delivery. Advanced drug delivery reviews 2006, 58, 1655-1670.

[54] Dai, S.; Ravi, P.; Tam, K. C. pH-Responsive polymers: synthesis, properties and applications. Soft Matter 2008, 4, 435-449.

[55] Gupta, P.; Vermani, K.; Garg, S. Hydrogels: from controlled release to $\mathrm{pH}^{-}$ responsive drug delivery. Drug discovery today 2002, 7, 569-579. 
[56] Lin, S.; Theato, P. CO2-responsive polymers. Macromolecular rapid communications 2013, 34, 1118-1133.

[57] Adhikari, B.; Majumdar, S. Polymers in sensor applications. Progress in polymer science 2004, 29, 699-766.

[58] Turkevich, J.; Stevenson, P. C.; Hillier, J. A study of the nucleation and growth processes in the synthesis of colloidal gold. Discussions of the Faraday Society 1951, $11,55-75$.

[59] Ninham, B. W. On progress in forces since the DLVO theory. Advances in colloid and interface science 1999, 83, 1-17.

[60] Zhang, X.; Servos, M. R.; Liu, J. Surface science of DNA adsorption onto citratecapped gold nanoparticles. Langmuir 2012, 28, 3896-3902.

[61] Porta, F.; Speranza, G.; Krpetic, Ž.; Dal Santo, V.; Francescato, P.; Scari, G. Gold nanoparticles capped by peptides. Materials Science and Engineering: B 2007, 140, 187-194.

[62] Brust, M.; Walker, M.; Bethell, D.; Schiffrin, D. J.; Whyman, R. Synthesis of thiolderivatised gold nanoparticles in a two-phase liquid-liquid system. Journal of the Chemical Society, Chemical Communications 1994, 801-802.

[63] Stöber, W.; Fink, A.; Bohn, E. Controlled growth of monodisperse silica spheres in the micron size range. Journal of colloid and interface science 1968, 26, 62-69.

[64] Ding, H.; Zhang, Y.; Wang, S.; Xu, J.; Xu, S.; Li, G. $\quad \mathrm{Fe}_{3} \mathrm{O}_{4} @ \mathrm{SiO}_{2} \quad$ core/shell nanoparticles: the silica coating regulations with a single core for different core sizes and shell thicknesses. Chemistry of Materials 2012, 24, 4572-4580.

[65] Cai, Y.; Peng, W.; Demeshko, S.; Tian, J.; Vana, P. Silica-coated magnetite nanoparticles carrying a high-density polymer brush shell of hydrophilic polymer. Macromolecular rapid communications 2018, 39, 1800226.

[66] Van Blaaderen, A.; Kentgens, A. Particle morphology and chemical microstructure of colloidal silica spheres made from alkoxysilanes. Journal of Non-Crystalline Solids 1992, 149, 161-178.

[67] Selvan, S. T.; Tan, T. T.; Ying, J. Y. Robust, non-cytotoxic, silica-coated CdSe quantum dots with efficient photoluminescence. Advanced Materials 2005, 17, 1620 1625.

[68] Zhuravlev, L. Concentration of hydroxyl groups on the surface of amorphous silicas. Langmuir 1987, 3, 316-318. 
[69] Guasch, J.; $\quad$ Diemer, J.; Riahinezhad, H.; Neubauer, S.; $\quad$ Kessler, H.; Spatz, J. P. Synthesis of binary nanopatterns on hydrogels for initiating cellular responses. Chemistry of Materials 2016, 28, 1806-1815.

[70] Lai, J. T.; Filla, D.; Shea, R. Functional polymers from novel carboxyl-terminated trithiocarbonates as highly efficient RAFT agents. Macromolecules 2002, 35, 67546756.

[71] Sebakhy, K. O.; Gavrilov, M.; Valade, D.; Jia, Z.; Monteiro, M. J. Nanoparticles of well-defined 4-arm stars made using nanoreactors in water. Macromolecular rapid communications 2014, 35, 193-197.

[72] Barner-Kowollik, C.; Buback, M.; Charleux, B.; Coote, M. L.; Drache, M.; Fukuda, T.; Goto, A.; Klumperman, B.; Lowe, A. B.; Mcleary, J. B., et al. Mechanism and kinetics of dithiobenzoate-mediated RAFT polymerization. I. The current situation. Journal of Polymer Science Part A: Polymer Chemistry 2006, 44, 5809-5831.

[73] Moad, G.; Chong, Y.; Postma, A.; Rizzardo, E.; Thang, S. H. Advances in RAFT polymerization: the synthesis of polymers with defined end-groups. Polymer 2005, 46, 8458-8468.

[74] Peng, W.; $\quad$ Rossner, C.; $\quad$ Roddatis, V.; Vana, P. Gold-planet-silver-satellite nanostructures using RAFT star polymer. ACS Macro Letters 2016, 5, 1227-1231.

[75] Baum, M.; Brittain, W. J. Synthesis of polymer brushes on silicate substrates via reversible addition fragmentation chain transfer technique. Macromolecules 2002, 35, 610-615.

[76] Li, C.; Han, J.; Ryu, C. Y.; Benicewicz, B. C. A versatile method to prepare RAFT agent anchored substrates and the preparation of PMMA grafted nanoparticles. Macromolecules 2006, 39, 3175-3183.

[77] Deng, J.; Zhao, C.; Spatz, J. P.; Wei, Q. Nanopatterned adhesive, stretchable hydrogel to control ligand spacing and regulate cell spreading and migration. ACS nano 2017, 11, 8282-8291.

[78] Cavalcanti-Adam, E. A.; Volberg, T.; Micoulet, A.; Kessler, H.; Geiger, B.; Spatz, J. P. Cell spreading and focal adhesion dynamics are regulated by spacing of integrin ligands. Biophysical journal 2007, 92, 2964-2974.

[79] Cavalcanti-Adam, E. A.; Aydin, D.; Hirschfeld-Warneken, V. C.; Spatz, J. P. Cell adhesion and response to synthetic nanopatterned environments by steering receptor clustering and spatial location. HFSP journal 2008, 2, 276-285. 
[80] Pimpin, A.; Srituravanich, W. Review on micro-and nanolithography techniques and their applications. Engineering Journal 2012, 16, 37-56.

[81] Vieu, C.; Carcenac, F.; Pepin, A.; Chen, Y.; Mejias, M.; Lebib, A.; Manin-Ferlazzo, L.; Couraud, L.; Launois, H. Electron beam lithography: resolution limits and applications. Applied surface science 2000, 164, 111-117.

[82] Chen, Y. Nanofabrication by electron beam lithography and its applications: A review. Microelectronic Engineering 2015, 135, 57-72.

[83] Piner, R. D.; Zhu, J.; Xu, F.; Hong, S.; Mirkin, C. A. "Dip-pen" nanolithography. science 1999, 283, 661-663.

[84] Garcia, R.; Knoll, A. W.; Riedo, E. Advanced scanning probe lithography. Nature nanotechnology 2014, 9, 577.

[85] Albisetti, E.; Petti, D.; Pancaldi, M.; Madami, M.; Tacchi, S.; Curtis, J.; King, W. P.; Papp, A.; Csaba, G.; Porod, W., et al. Nanopatterning reconfigurable magnetic landscapes via thermally assisted scanning probe lithography. Nature nanotechnology 2016, 11, 545.

[86] Pires, D.; $\quad$ Hedrick, J. L.; $\quad$ De Silva, A.; $\quad$ Frommer, J.; $\quad$ Gotsmann, B.; Wolf, H.; Despont, M.; Duerig, U.; Knoll, A.W. Nanoscale three-dimensional patterning of molecular resists by scanning probes. Science 2010, 328, 732-735.

[87] Chou, S. Y.; Krauss, P. R.; Renstrom, P. J. Imprint lithography with 25-nanometer resolution. Science 1996, 272, 85-87.

[88] Gates, B. D.; Xu, Q.; Stewart, M.; Ryan, D.; Willson, C. G.; Whitesides, G. M. New approaches to nanofabrication: molding, printing, and other techniques. Chemical reviews 2005, 105, 1171-1196.

[89] Kumar, T. A.; Bardea, A.; Shai, Y.; Yoffe, A.; Naaman, R. Patterning gradient properties from sub-micrometers to millimeters by magnetolithography. Nano letters 2010, 10, 2262-2267.

[90] Spatz, J. P.; $\quad$ Mössmer, S.; Hartmann, C.; Möller, M.; Herzog, T.; Krieger, M.; Boyen, H.-G.; Ziemann, P.; Kabius, B. Ordered deposition of inorganic clusters from micellar block copolymer films. Langmuir 2000, 16, 407-415.

[91] Cheung, C. L.; Nikolic, R.; Reinhardt, C.; Wang, T. Fabrication of nanopillars by nanosphere lithography. Nanotechnology 2006, 17, 1339. 
[92] Manfrinato, V. R.; $\quad$ Zhang, L.; $\quad$ Su, D.; $\quad$ Duan, H.; $\quad$ Hobbs, R. G.; $\quad$ Stach, E. A.; Berggren, K. K. Resolution limits of electron-beam lithography toward the atomic scale. Nano letters 2013, 13, 1555-1558.

[93] Salaita, K.; Wang, Y.; Mirkin, C. A. Applications of dip-pen nanolithography. Nature nanotechnology 2007, 2, 145.

[94] Bat, E.; Lee, J.; Lau, U. Y.; Maynard, H. D. Trehalose glycopolymer resists allow direct writing of protein patterns by electron-beam lithography. Nature communications 2015, 6, 6654 .

[95] Harnett, C.; Satyalakshmi, K.; Craighead, H. Bioactive templates fabricated by lowenergy electron beam lithography of self-assembled monolayers. Langmuir 2001, 17, 178-182.

[96] Hoff, J. D.; Cheng, L.-J.; Meyhöfer, E.; Guo, L. J.; Hunt, A. J. Nanoscale protein patterning by imprint lithography. Nano letters 2004, 4, 853-857.

[97] Arnold, M.; Cavalcanti-Adam, E. A.; Glass, R.; Blümmel, J.; Eck, W.; Kantlehner, M.; Kessler, H.; Spatz, J. P. Activation of integrin function by nanopatterned adhesive interfaces. ChemPhysChem 2004, 5, 383-388.

[98] Glass, R.; Möller, M.; Spatz, J. P. Block copolymer micelle nanolithography. Nanotechnology 2003, 14, 1153.

[99] Ohno, K.; Akashi, T.; Huang, Y.; Tsujii, Y. Surface-initiated living radical polymerization from narrowly size-distributed silica nanoparticles of diameters less than $100 \mathrm{~nm}$. Macromolecules 2010, 43, 8805-8812.

[100] Spatz, J. P.; Mößmer, S.; Möller, M. Mineralization of gold nanoparticles in a block copolymer microemulsion. Chemistry-A European Journal1996, 2, 1552-1555.

[101] Schenk, F. C.; $\quad$ Boehm, H.; Spatz, J. P.; $\quad$ Wegner, S. V. $\quad$ Dual-functionalized nanostructured biointerfaces by click chemistry. Langmuir 2014, 30, 6897-6905.

[102] Boyen, H.-G.; Herzog, T.; Kästle, G.; Weigl, F.; Ziemann, P.; Spatz, J. P.; Möller, M.; Wahrenberg, R.; Garnier, M.; Oelhafen, P. X-ray photoelectron spectroscopy study on gold nanoparticles supported on diamond. Physical Review B 2002, 65, 075412.

[103] Lee, B. K.; Lee, H. Y.; Kim, P.; Suh, K. Y.; Seo, J. H.; Cha, H. J.; Kawai, T. Stepwise selfassembly of a protein nanoarray from a nanoimprinted poly (Ethylene Glycol) hydrogel. small 2008, 4, 342-348.

[104] Engler, A. J.; Sen, S.; Sweeney, H. L.; Discher, D. E. Matrix elasticity directs stem cell lineage specification. Cell2006, 126, 677-689. 
[105] Aydin, D.; Louban, I.; Perschmann, N.; Blümmel, J.; Lohmüller, T.; CavalcantiAdam, E. A.; Haas, T. L.; Walczak, H.; Kessler, H.; Fiammengo, R., et al. Polymeric substrates with tunable elasticity and nanoscopically controlled biomolecule presentation. Langmuir 2010, 26, 15472-15480.

[106] Graeter, S. V.; Huang, J.; Perschmann, N.; López-Garcá, M.; Kessler, H.; Ding, J.; Spatz, J. P. Mimicking cellular environments by nanostructured soft interfaces. Nano letters 2007, 7, 1413-1418.

[107] Lohmüller, T.; Aydin, D.; Schwieder, M.; Morhard, C.; Louban, I.; Pacholski, C.; Spatz, J.P. Nanopatterning by block copolymer micelle nanolithography and bioinspired applications. Biointerphases 2011, 6, MR1-MR12.

[108] Gunawidjaja, R.; Peleshanko, S.; Ko, H.; Tsukruk, V. V. Bimetallic nanocobs: decorating silver nanowires with gold nanoparticles. Advanced Materials 2008, 20, 1544-1549.

[109] Chen, H.; Wang, F.; Li, K.; Woo, K. C.; Wang, J.; Li, Q.; Sun, L.-D.; Zhang, X.; Lin, H.Q.; Yan, C.-H. Plasmonic percolation: plasmon-manifested dielectric-to-metal transition. ACS nano 2012, 6, 7162-7171.

[110] Lim, B.; Jiang, M.; Camargo, P. H.; Cho, E. C.; Tao, J.; Lu, X.; Zhu, Y.; Xia, Y. Pd-Pt bimetallic nanodendrites with high activity for oxygen reduction. science 2009, 324, 1302-1305.

[111] Chang, H.; Kang, H.; Yang, J.-K.; Jo, A.; Lee, H.-Y.; Lee, Y.-S.; Jeong, D. H. Ag shell-Au satellite hetero-nanostructure for ultra-sensitive, reproducible, and homogeneous NIR SERS activity. ACS applied materials \& interfaces 2014, 6, 11859-11863.

[112] Li, A.; Tang, L.; Song, D.; Song, S.; Ma, W.; Xu, L.; Kuang, H.; Wu, X.; Liu, L.; Chen, X., et al. A SERS-active sensor based on heterogeneous gold nanostar core-silver nanoparticle satellite assemblies for ultrasensitive detection of aflatoxinB1. Nanoscale 2016, 8, 1873-1878.

[113] Feng, J.; Wu, X.; Ma, W.; Kuang, H.; Xu, L.; Xu, C. A SERS active bimetallic coresatellite nanostructure for the ultrasensitive detection of Mucin-1. Chemical Communications 2015, 51, 14761-14763.

[114] Wang, X.; Wang, Y.; Zhu, J.; Xu, Y. Hierarchical AgNR@ Cys@ AuNPs helical coresatellite nanostructure: Shape-dependent assembly and chiroptical response. The Journal of Physical Chemistry C 2014, 118, 5782-5788.

[115] Rossner, C.; Vana, P. Controlled Radical Polymerization at and from Solid Surfaces; Springer, 2015; pp 193-220. 
[116] Götz, T.; Hoheisel, W.; Vollmer, M.; Träger, F. Characterization of large supported metal clusters by optical spectroscopy. Zeitschrift für Physik D Atoms, Molecules and Clusters 1995, 33, 133-141.

[117] Zheng, Y.; Thai, T.; Reineck, P.; Qiu, L.; Guo, Y.; Bach, U. DNA-directed selfassembly of core-satellite plasmonic nanostructures: A highly sensitive and reproducible near-IR SERS sensor. Advanced Functional Materials 2013, 23, 15191526.

[118] Zhou, Q.; Li, X.; Fan, Q.; Zhang, X.; Zheng, J. Charge transfer between metal nanoparticles interconnected with a functionalized molecule probed by surfaceenhanced Raman spectroscopy. Angewandte Chemie International Edition 2006, 45, 3970-3973.

[119] Yoon, J. H.; Zhou, Y.; Blaber, M. G.; Schatz, G. C.; Yoon, S. Surface plasmon coupling of compositionally heterogeneous core-satellite nanoassemblies. The journal of physical chemistry letters 2013, 4, 1371-1378.

[120] Mucic, R. C.; Storhoff, J. J.; Mirkin, C. A.; Letsinger, R. L. DNA-directed synthesis of binary nanoparticle network materials. Journal of the American Chemical Society 1998 , 120, 12674-12675.

[121] Sebba, D.; LaBean, T.; Lazarides, A. Plasmon coupling in binary metal coresatellite assemblies. Applied Physics B 2008, 93, 69.

[122] De las Heras Alarcón, C.; Pennadam, S.; Alexander, C. Stimuli responsive polymers for biomedical applications. Chemical Society Reviews 2005, 34, 276-285.

[123] Nath, N.; Chilkoti, A. Creating "smart" surfaces using stimuli responsive polymers. Advanced materials 2002, 14, 1243-1247.

[124] Bawa, P.; Pillay, V.; Choonara, Y. E.; Du Toit, L. C. Stimuli-responsive polymers and their applications in drug delivery. Biomedical materials 2009, 4, 022001.

[125] Shim, M. S.; Kwon, Y. J. Stimuli-responsive polymers and nanomaterials for gene delivery and imaging applications. Advanced drug delivery reviews 2012, 64, 10461059.

[126] Gupta, S.; Agrawal, M.; Uhlmann, P.; Simon, F.; Oertel, U.; Stamm, M. Gold nanoparticles immobilized on stimuli responsive polymer brushes as nanosensors. Macromolecules 2008, 41, 8152-8158.

[127] Chiefari, J.; Chong, Y.; Ercole, F.; Krstina, J.; Jeffery, J.; Le, T. P.; Mayadunne, R. T.; Meijs, G. F.; Moad, C. L.; Moad, G., et al. Living free-radical polymerization by reversible 
addition- fragmentation chain transfer: the RAFT process. Macromolecules 1998, 31, 5559-5562.

[128] Hasell, T.; Thurecht, K. J.; Jones, R. D.; Brown, P. D.; Howdle, S. M. Novel one pot synthesis of silver nanoparticle-polymer composites by supercritical CO_2 polymerisation in the presence of a RAFT agent. Chemical communications 2007, 3933-3935.

[129] Sun, Y.; Liu, Y.; Zhao, G.; Zhou, X.; Gao, J.; Zhang, Q. Preparation of pH-responsive silver nanoparticles by RAFT polymerization. Journal of materials science 2008, 43 , 4625-4630.

[130] Rossner, C.; Ebeling, B.; Vana, P. Spherical gold-nanoparticle assemblies with tunable interparticle distances mediated by multifunctional RAFT polymers. ACS Macro Letters 2013, 2, 1073-1076.

[131] Boschmann, D.; Edam, R.; Schoenmakers, P. J.; Vana, P. Characterization of ZRAFT star polymerization of butyl acrylate by size-exclusion chromatography. 2009, $275,184-196$.

[132] Hiramatsu, H.; Osterloh, F. E. A simple large-scale synthesis of nearly monodisperse gold and silver nanoparticles with adjustable sizes and with exchangeable surfactants. Chemistry of Materials 2004, 16, 2509-2511.

[133] Fink, J.; Kiely, C. J.; Bethell, D.; Schiffrin, D. J. Self-organization of nanosized gold particles. Chemistry of materials 1998, 10, 922-926.

[134] Tan, S. J.; $\quad$ Campolongo, M. J.; $\quad$ Luo, D.; $\quad$ Cheng, W. Building $\quad$ plasmonic nanostructures with DNA. Nature nanotechnology2011, 6, 268.

[135] Jain, P. K.; Huang, W.; El-Sayed, M. A. On the universal scaling behavior of the distance decay of plasmon coupling in metal nanoparticle pairs: a plasmon ruler equation. Nano Letters 2007, 7, 2080-2088.

[136] Asher, S. A.; Holtz, J.; Weissman, J.; Pan, G. Mesoscopically periodic photoniccrystal materials for linear and nonlinear optics and chemical sensing. Mrs Bulletin 1998, 23, 44-50.

[137] Amschler, K.; Erpenbeck, L.; Kruss, S.; Scho 虉 n, M. P. Nanoscale integrin ligand patterns determine melanoma cell behavior. ACS nano 2014, 8, 9113-9125.

[138] Rossetti, F. F.; Reviakine, I.; Csúcs, G.; Assi, F.; Vörös, J.; Textor, M. Interaction of poly (L-lysine)-g-poly (ethylene glycol) with supported phospholipid bilayers. Biophysical journal 2004, 87, 1711-1721. 
[139] Wagner, J.; Peng, W.; Vana, P. Polyethylene-grafted gold and silver nanoparticles using catalyzed chain growth (CCG). Polymers 2018, 10, 407.

[140] Damiron, D.; Mazzolini, J.; Cousin, F.; Boisson, C.; d'Agosto, F.; Drockenmuller, E. Poly (ethylene) brushes grafted to silicon substrates. Polymer Chemistry 2012, 3, 1838-1845.

[141] Bieligmeyer, M.; Taheri, S. M.; German, I.; Boisson, C.; Probst, C.; Milius, W.; Altstädt, V.; Breu, J.; Schmidt, H.-W.; D'agosto, F., et al. Completely miscible polyethylene nanocomposites. Journal of the American Chemical Society 2012, 134, 18157-18160.

[142] Godoy Lopez, R.; Boisson, C.; d'Agosto, F.; Spitz, R.; Boisson, F.; Gigmes, D.; Bertin, D. New functional polyolefins: Towards a bridge between catalytic and RAFT polymerizations? Macromolecular rapid communications 2006, 27, 173-181.

[143] Xiao, L.; Li, J.; Brougham, D. F.; Fox, E. K.; Feliu, N.; Bushmelev, A.; Schmidt, A.; Mertens, N.; Kiessling, F.; Valldor, M.; Fadeel, B.; Mathur, S. Water-soluble superparamagnetic magnetite nanoparticles with biocompatible coating for enhanced magnetic resonance imaging. ACS Nano 2011, 5, 6315-6324.

[144] Sun, C.; Lee, J. S.; Zhang, M. Magnetic nanoparticles in MR imaging and drug delivery. Adv. Drug Delivery Rev. 2008, 60, 1252-1265.

[145] Wang, Y.-X. J.; Hussain, S. M.; Krestin, G. P. Superparamagnetic iron oxide contrast agents: physicochemical characteristics and applications in MR imaging. Eur. Radiol. 2001, 11, 2319-2331.

[146] Tromsdorf, U. I.; Bruns, O. T.; Salmen, S. C.; Beisiegel, U.; Weller, H. A highly effective, nontoxic $T_{1}$ MR contrast agent based on ultrasmall PEGylated iron oxide nanoparticles. Nano Lett. 2009, 9, 4434-4440.

[147] Sun, C.; Du, K.; Fang, C.; Bhattarai, N.; Veiseh, O.; Kievit, F.; Stephen, Z.; Lee, D.; Ellenbogen, R. G.; Ratner, B.; Zhang, M. PEG-mediated synthesis of highly dispersive multifunctional superparamagnetic nanoparticles: their physicochemical properties and function in vivo. ACS Nano 2010, 4, 2402-2410.

[148] Huang, J.; Bu, L.; Xie, J.; Chen, K.; Cheng, Z.; Li, X.; Chen, X. Effects of nanoparticle size on cellular uptake and liver MRI with polyvinylpyrrolidone-coated iron oxide nanoparticles. ACS Nano 2010, 4, 7151-7160.

[149] Chen, Y.; Chen, H.; Zeng, D.; Tian, Y.; Chen, F.; Feng, J.; Shi, J. Core/shell structured hollow mesoporous nanocapsules: a potential platform for simultaneous cell imaging and anticancer drug delivery. ACS Nano 2010, 4, 6001-6013. 
[150] Park, J.; Kadasala, N. R.; Abouelmagd, S. A.; Castanares, M. A.; Collins, D. S.; Wei, A.; Yeo, Y. Polymer-iron oxide composite nanoparticles for EPR-independent drug delivery. Biomaterials 2016, 101, 285-295.

[151] Ulbrich, K.; Holá, K.; Subr, V.; Bakandritsos, A.; Tucek, J.; Zboril, R. Targeted drug delivery with polymers and magnetic nanoparticles: covalent and noncovalent approaches, release control, and clinical studies. Chem. Rev. 2016, 116, 5338-5431.

[152] Zanganeh, S.; $\quad$ Hutter, G.; $\quad$ Spitler, R.; Lenkov, O.; Mahmoudi, M.; Shaw, A.; Pajarinen, J. S.; Nejadnik, H.; Goodman, S.; Moseley, M.; Coussens, L. M.; DaldrupLink, H.E. Iron oxide nanoparticles inhibit tumour growth by inducing proinflammatory macrophage polarization in tumour tissues. Nat. Nanotechnol. 2016, 11, 986.

[153] Maier-Hauff, K.; Ulrich, F.; Nestler, D.; Niehoff, H.; Wust, P.; Thiesen, B.; Orawa, H.; Budach, V.; Jordan, A. Efficacy and safety of intratumoral thermotherapy using magnetic iron-oxide nanoparticles combined with external beam radiotherapy on patients with recurrent glioblastoma multiforme. J. Neurooncol. 2011, 103, 317-324.

[154] Andreu, I.; Natividad, E.; Solozabal, L.; Roubeau, O. Nano-objects for addressing the control of nanoparticle arrangement and performance in magnetic hyperthermia. ACS Nano 2015, 9, 1408-1419.

[155] Bazak, R.; Houri, M.; El Achy, S.; Kamel, S.; Refaat, T. Cancer active targeting by nanoparticles: a comprehensive review of literature. J. Cancer Res. Clin. Oncol. 2015, 141, 769-784.

[156] Ohno, K.; $\quad$ Mori, C.; Akashi, T.; Yoshida, S.; Tago, Y.; Tsujii, Y.; Tabata, Y. Fabrication of contrast agents for magnetic resonance imaging from polymer-brushafforded iron oxide magnetic nanoparticles prepared by surface-initiated living radical polymerization. Biomacromolecules 2013, 14, 3453-3462.

[157] Lu, A.-H.; Salabas, E. e.; Schüth, F. Magnetic nanoparticles: synthesis, protection, functionalization, and application. Angew. Chem. Int. Ed. 2007, 46, 1222-1244.

[158] Davila-Ibanez, A. B.; Salgueirino, V.; Martinez-Zorzano, V.; Mariño Fernández, R.; García-Lorenzo, A.; Maceira-Campos, M.; Muñoz Ubeda, M.; Junquera, E.; Aicart, E.; Rivas, J. Magnetic silica nanoparticle cellular uptake and cytotoxicity regulated by electrostatic polyelectrolytes-DNA loading at their surface. ACS Nano 2011, 6, 747-759. [159] Han, Y.; Jiang, J.; Lee, S. S.; Ying, J. Y. Reverse microemulsion-mediated synthesis of silica-coated gold and silver nanoparticles. Langmuir 2008, 24, 5842-5848. 
[160] Granitzer, P.; Rumpf, K.; Roca, A.; Morales, M.; Poelt, P.; Albu, M. Investigation of a mesoporous silicon based ferromagnetic nanocomposite. Nanoscale Res. Lett. 2010, $5,374$.

[161] Garcá-Otero, J.; Porto, M.; Rivas, J.; Bunde, A. Influence of dipolar interaction on magnetic properties of ultrafine ferromagnetic particles. Phys. Rev. Lett. 2000, 84, 167. [162] Woinska, M.; Szczytko, J.; Majhofer, A.; Gosk, J.; Dziatkowski, K.; Twardowski, A. Magnetic interactions in an ensemble of cubic nanoparticles: A Monte Carlo study. Phys. Rev. B2013, 88, 144421.

[163] Huang, Y.; Zheng, Y.; Sarkar, A.; Xu, Y.; Stefik, M.; Benicewicz, B. C. Matrix-free polymer nanocomposite thermoplastic elastomers. Macromolecules 2017, 50, 47424753.

[164] Jewett, J. C.; Sletten, E. M.; Bertozzi, C. R. Rapid Cu-free click chemistry with readily synthesized biarylazacyclooctynones. Journal of the American Chemical Society 2010, 132, 3688-3690. 


\section{Acknowledgements}

First of all, I would like to express my deepest gratitude to professor Philipp Vana for convincingly guiding and encouraging me during the whole $\mathrm{PhD}$ period. Without his strong support and persistent help, this project would not have been realized.

I also want to thank my co-supervisor professor Alec Wodtke for his valuable discussion and interest in my topic.

Professor Michael Buback, professor Ricardo Mata, professor Andreas Janshoff and professor Sven Schneider are gratefully acknowledged for being members of my thesis committee.

It's my luck and honor that I had the opportunity to collaborate with many amazing people during my $\mathrm{PhD}$ period. I am very thankful to professor Michael Schön and Dr. Katharina Amschler from the university clinic of Göttingen for the cell experiment and many thoughtful discussions. Professor Fraucke Alves from Max-Planck institute for experimental medicine in Göttingen and Dr. Sebastian Kruss from the university of Göttingen their gratefully acknowledged for their help and discussion in the aspect of biology. Professor Lutz Ackermann and Santhi Vardhana Reddy Yetra for the cooperation on a project on micellar catalysis. I would like to thank Dr. Vladimir for performing the HAADF and HRTEM experiment. Dr. Serhiy Demeshko has performed SQUID experiment for the magnetite project which gives a detailed insight into the improvement of magnetic properties using our method. I want to thank Matthias Hahn and Volker Radisch from the institute for material physics for assistance on TEM and SEM. I am also very grateful to professor Akito Masuhara and Keiji Shito from Yamagata University for the support and interesting discussions during the conference in Japan. 
Generous support from the DFG (project number VA226-10/1) and the Georg-August-Universität Göttingen are gratefully acknowledged.

I am very grateful to Yingying Cai, without her inspiration, meticulous observation and expertise on the very detail of the experiment, the polymerization on silica will never proceed perfectly. The devil is in the detail, her sharp eyes always spot the tiny devil when the experiments met obstacles and helped me through the most difficult part of this thesis.

I am very thankful to Dr. Jannik Wagner. Without his expertise in polyethylene synthesis, the PE capped gold and silver nanoparticles will probably not exist in this world to the date of this thesis.

I would also like to acknowledge Christian Fricke, Stella Weber, Qingyuan Song for being my trainees and supported my experiments.

I want to thank all members of the MMC group especially my officemates and ex-officemates Alex Groschopp, Jannik Wagner, Judith Steinhoff for their contributing to a pleasant working atmosphere.

I want to give my thanks to Lara Riemann, Judith Steinhoff, Yingying Cai and myself for carefully proofreading this thesis and very useful suggestions.

Special thanks go to Yingying Cai, Stella Weber, and Kokubo Shinsuke for the creation of the cover picture for the Macro Letter paper.

Finally, I'd like to be thankful to my family and friends who support and uplift me, especially my lovely wife Yingying Cai. Together, we're strong! 


\section{Curriculum Vitae}

\section{Personal data}

Name

Wentao Peng

Place of birth

Jiaxing, China

Date of birth

9. Jun. 1989

Address

Steinweg 24

37120 Bovenden

Nationality

Chinese

Email:

wentaopengcustom@gmail.com

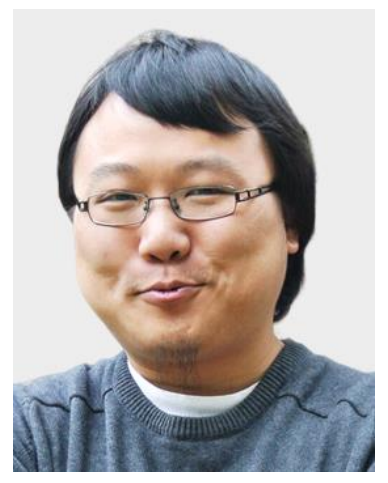

\section{Education}

\begin{tabular}{|c|c|}
\hline $01.2016-03.2020$ & $\begin{array}{l}\text { Georg-August-Universität Göttingen, Doktor rer. Nat., } \\
\text { (equiv. to PhD) }\end{array}$ \\
\hline & $\begin{array}{l}\text { Thesis: "Binary Planet-Satellite Nanostructure Using } \\
\text { RAFT Polymer" } \\
\text { Supervisor: Prof. Dr. Philipp Vana MBA, co-referent; Prof. } \\
\text { Ph.D. Alec Michael Wodtke }\end{array}$ \\
\hline $09.2013-09.2015$ & $\begin{array}{l}\text { Georg-August-Universität Göttingen, M. Sc., in Chemistry } \\
\text { (Grade: "sehr gut" (very good) 1.5) } \\
\text { Thesis: "Functionalization of noble metal } \\
\text { nanocomposites" } \\
\text { Supervisor: Prof. Dr. Philipp Vana MBA }\end{array}$ \\
\hline $10.2010-07.2013$ & $\begin{array}{l}\text { Georg-August-Universität Göttingen, B. Sc., in Chemistry } \\
\text { (Grade: "gut" (good) 1.7) } \\
\text { Thesis: "Mechanical properties and testing of polymers" } \\
\text { Supervisor: Prof. Dr. Philipp Vana MBA }\end{array}$ \\
\hline $09.2009-07.2010$ & German courses, Tongji University, Shanghai, China \\
\hline $09.2008-06.2009$ & $\begin{array}{l}\text { University of Shanghai, Shanghai, China, Applied } \\
\text { Chemistry (finished } 3 \text { semesters, grade: } 1.9 \text { ) }\end{array}$ \\
\hline $09.2005-07.2008$ & $\begin{array}{l}\text { High school graduation, Jiaxing No.1 Middle School, } \\
\text { Jiaxing, China }\end{array}$ \\
\hline
\end{tabular}




\section{Scholarship}

2012 - $2013 \quad$ August-Wilhelm-von-Hofmann-Stiftung (GDCh)

09. $2011 \quad$ Niedersachsenstipendium

$2008-2009 \quad$ The first prize scholarship of University of Shanghai

$09.2008 \quad$ Special freshman scholarship of University of Shanghai

\section{Professional experience}

11.2014 - $02.2015 \quad$ Sartorius Stedim Biotech, Trainee in the R\&D department of membrane technology

$2012-2020$ Georg-August Universität Göttingen, tutor for diverse course and experiments

\section{Language skills}

Chinese Native speaker

German Near native (C1-Niveau im Feb. 2010)

English Near native

Japanese Excellent command

\section{Publications}

$\diamond$ "Micellar catalysis for Ruthenium(II)-catalyzed C-H arylations: Weak coordination-enabled C-H activation in $\mathrm{H}_{2} \mathrm{O}$ " S. R. Yetra, T. Rogge, S. Warratz, J. Struwe, W. Peng, P. Vana, L. Ackermann Angewandte Chemie International Edition 2019, 58, 7490.

$\diamond \quad$ "Silica-coated magnetite nanoparticles carrying a high-density polymer brush shell of hydrophilic polymer" Y. Cai, W. Peng, S. Demeshko, J. Tian, P. Vana Macromolecular Rapid Communications 2018, 39, 1800226.

$\diamond \quad$ "Polyethylene-covered gold and silver nanoparticles using catalyzed chain growth (CCG)” J. Wagner, W. Peng, P. Vana Polymers 2018, 10, 407.

$\diamond \quad$ "Gold-planet-silver-satellite nanostructures using RAFT star polymer", W. Peng, C. Rossner, V. Roddatis, P. Vana ACS Macro Letters 2016, 5, 1227-1231. 
$\diamond$ "Titin-Mimicking Polycyclic Polymers with Shape Regeneration and Healing Properties" J.-H. Schuetz, W. Peng, P. Vana Polymer Chemistry 2015, 6, 1714-1726.

\section{Presentations}

$\diamond \quad$ "Binary planet-satellite nanostructures using RAFT polymer", 22nd International Conference on Advanced Materials and Nanotechnology, Tokyo, Japan Sep. 2018

$\diamond$ "Creating planet-satellite nanoassemblies with functional polymer", 18th International Symposium on Advanced Organic Photonics, Yonezawa, Japan Sep. 2018

$\diamond \quad$ "Nanokosmos: Planet-Satelliten Nanohybrid aus Gold und Silber", Jung Chemiker Forum, Göttingen, Germany, 2016. 